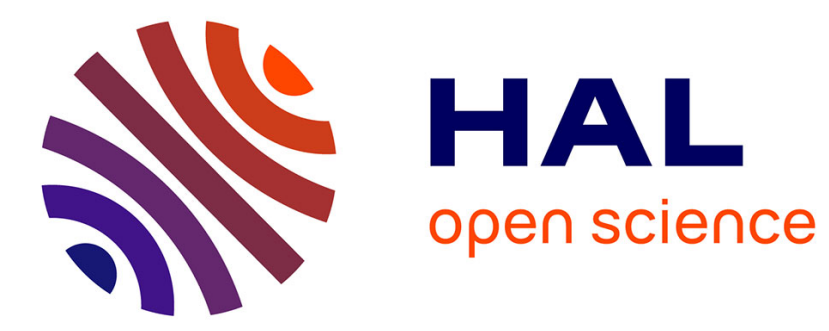

\title{
Precipitation and abnormal grain growth in low alloy steels
}

\author{
Mohammad Razzak
}

\section{To cite this version:}

Mohammad Razzak. Precipitation and abnormal grain growth in low alloy steels. Materials. INSA de Lyon, 2013. English. NNT: 2013ISAL0094 . tel-01015867

\section{HAL Id: tel-01015867 \\ https://theses.hal.science/tel-01015867}

Submitted on 27 Jun 2014

HAL is a multi-disciplinary open access archive for the deposit and dissemination of scientific research documents, whether they are published or not. The documents may come from teaching and research institutions in France or abroad, or from public or private research centers.
L'archive ouverte pluridisciplinaire HAL, est destinée au dépôt et à la diffusion de documents scientifiques de niveau recherche, publiés ou non, émanant des établissements d'enseignement et de recherche français ou étrangers, des laboratoires publics ou privés. 


\title{
THĖSE
}

\section{Precipitation and abnormal grain growth in low alloy steels}

\author{
PRÉSENTÉE DEVANT \\ L'Institut National Des Sciences Appliquées de \\ LYON \\ POUR OBTENIR \\ LE GRADE DE DOCTEUR \\ ECole doctorale: Matériaux de Lyon \\ Spécialité: MatériauX \\ PAR \\ Mohammad ABDur RAZZAK
}

Soutenance le 4 Octobre 2013 devant la Commission d'examen

Jury
Anthony D. ROLLETT
Examinateur
Ernst KOZESCHNIK
Rapporteur
Philippe MAUGIS
Rapporteur
Michel PEREZ
Directeur de thèse
Sophie CAZOTTES
Directeur de thèse
Thomas SOURMAIL
Examinateur
Marion FROTEY
Invité 
Cette thèse est accessible à l'adresse : http://theses.insa-lyon.fr/publication/2013ISAL0094/these.pdf () [M.A. Razzak], [2013], INSA de Lyon, tous droits réservés 
INSA Direction de la Recherche - Ecoles Doctorales - Quinquennal 2011-2015

\begin{tabular}{|c|c|c|}
\hline SIGLE & ECOLE DOCTORALE & NOM ET COORDONNEES DU RESPONSABLE \\
\hline CHIMIE & $\begin{array}{l}\text { CHIMIE DE LYON } \\
\text { http://www.edchimie-lyon.fr } \\
\text { Insa : R. GOURDON }\end{array}$ & $\begin{array}{l}\text { M. Jean Marc LANCELIN } \\
\text { Université de Lyon - Collège Doctoral } \\
\text { Bât ESCPE } \\
43 \text { bd du } 11 \text { novembre } 1918 \\
69622 \text { VILLEURBANNE Cedex } \\
\text { Tél : 04.72.43 } 1395 \\
\text { directeur@edchimie-lyon.fr }\end{array}$ \\
\hline E.E.A. & $\begin{array}{l}\text { ELECTRONIQUE, } \\
\text { ELECTROTECHNIQUE, AUTOMATIQUE } \\
\text { http://edeea.ec-lyon.fr } \\
\text { Secrétariat : M.C. HAVGOUDOUKIAN } \\
\text { eea@ec-lyon.fr }\end{array}$ & $\begin{array}{l}\text { M. Gérard SCORLETTI } \\
\text { Ecole Centrale de Lyon } \\
36 \text { avenue Guy de Collongue } \\
\text { 69134 ECULLY } \\
\text { Té1: 04.72.1865 55 Fax : } 0478433717 \\
\text { Gerard.scorletti@ec-lyon.fr }\end{array}$ \\
\hline E2M2 & $\begin{array}{l}\text { EVOLUTION, ECOSYSTEME, } \\
\text { MICROBIOLOGIE, MODELISATION } \\
\underline{\text { http://e2m2.universite-lyon.fr }} \\
\text { Insa : H. CHARLES }\end{array}$ & $\begin{array}{l}\text { Mme Gudrun BORNETTE } \\
\text { CNRS UMR 5023 LEHNA } \\
\text { Université Claude Bernard Lyon } 1 \\
\text { Bât Forel } \\
43 \text { bd du } 11 \text { novembre } 1918 \\
69622 \text { VILLEURBANNE Cédex } \\
\text { Tél: 06.07.53.89.13 } \\
\text { e2m2@, univ-lyon1.fr }\end{array}$ \\
\hline EDISS & $\begin{array}{l}\text { INTERDISCIPLINAIRE SCIENCES- } \\
\underline{\text { SANTE }} \\
\text { http://www.ediss-lyon.fr } \\
\text { Sec : Samia VUILLERMOZ } \\
\text { Insa : M. LAGARDE }\end{array}$ & $\begin{array}{l}\text { M. Didier REVEL } \\
\text { Hôpital Louis Pradel } \\
\text { Bâtiment Central } \\
\text { 28 Avenue Doyen Lépine } \\
69677 \text { BRON } \\
\text { Tél:04.72.68.49.09 Fax :04 72 } 684916 \\
\text { Didier.revel@creatis.uni-lyon1.fr }\end{array}$ \\
\hline INFOMATHS & $\begin{array}{l}\text { INFORMATIQUE ET } \\
\text { MATHEMATIQUES } \\
\text { http://infomaths.univ-lyon1.fr } \\
\text { Sec :Renée EL MELHEM }\end{array}$ & $\begin{array}{l}\text { Mme Sylvie CALABRETTO } \\
\text { Université Claude Bernard Lyon } 1 \\
\text { INFOMATHS } \\
\text { Bâtiment Braconnier } \\
43 \text { bd du 11 novembre } 1918 \\
\text { 69622 VILLEURBANNE Cedex } \\
\text { Tél : 04.72. 44.82.94 Fax 04 } 72431687 \\
\text { infomaths@univ-lyon1.fr }\end{array}$ \\
\hline Matériaux & $\begin{array}{l}\text { MATERIAUX DE LYON } \\
\text { http://ed34.universite-lyon.fr } \\
\text { Secrétariat : M. LABOUNE } \\
\text { PM : } 71.70 \text {-Fax : } 87.12 \\
\text { Bat. Saint Exupéry } \\
\text { Ed.materiaux@insa-Iyon.fr }\end{array}$ & $\begin{array}{l}\text { M. Jean-Yves BUFFIERE } \\
\text { INSA de Lyon } \\
\text { MATEIS } \\
\text { Bâtiment Saint Exupéry } \\
7 \text { avenue Jean Capelle } \\
\text { 69621 VILLEURBANNE Cedex } \\
\text { Tél : 04.72.438318 Fax 04 72 } 438528 \\
\text { Jean-yves.buffiere@insa-lyon.fr }\end{array}$ \\
\hline MEGA & $\begin{array}{l}\text { MECANIQUE, ENERGETIQUE, GENIE } \\
\text { CIVIL, ACOUSTIQUE } \\
\text { http://mega.ec-lyon.fr } \\
\text { Secrétariat : M. LABOUNE } \\
\text { PM : } 71.70-\text {-Fax : } 87.12 \\
\text { Bat. Saint Exupéry } \\
\text { mega@insa-lyon.fr }\end{array}$ & $\begin{array}{l}\text { M. Philippe BOISSE } \\
\text { INSA de Lyon } \\
\text { Laboratoire LAMCOS } \\
\text { Bâtiment Jacquard } \\
\text { 25 bis avenue Jean Capelle } \\
69621 \text { VILLEURBANNE Cedex } \\
\text { Tél :04.72.43.71.70 Fax : } 0472437237 \\
\text { Philippe.boisse@insa-lyon.fr }\end{array}$ \\
\hline ScSo & $\begin{array}{l}\frac{\text { ScSo* }}{\text { http://recherche.univ-lyon2.fr/scso/ }} \\
\text { Sec : Viviane POLSINELLI } \\
\quad \text { Brigitte DUBOIS } \\
\text { Insa : J.Y. TOUSSAINT }\end{array}$ & $\begin{array}{l}\text { M. OBADIA Lionel } \\
\text { Université Lyon } 2 \\
86 \text { rue Pasteur } \\
69365 \text { LYON Cedex } 07 \\
\text { Tél : 04.78.77.23.86 Fax : 04.37.28.04.48 } \\
\text { Lionel.Obadia@univ-lyon2.fr }\end{array}$ \\
\hline
\end{tabular}

\footnotetext{
*ScSo : Histoire, Géographie, Aménagement, Urbanisme, Archéologie, Science politique, Sociologie, Anthropologie
} 
Cette thèse est accessible à l'adresse : http://theses.insa-lyon.fr/publication/2013ISAL0094/these.pdf () [M.A. Razzak], [2013], INSA de Lyon, tous droits réservés 
I dedicate this thesis to my father Mohammad Abdur Rauf Miaji, my mother Ferdousi Begum, my two sisters (Farjana Shumy and Farjin Sushmita) and to my wife Montahar Ahmed. 
Cette thèse est accessible à l'adresse : http://theses.insa-lyon.fr/publication/2013ISAL0094/these.pdf () [M.A. Razzak], [2013], INSA de Lyon, tous droits réservés 


\section{Acknowledgements}

This has been a long journey and fortunately I have been blessed with help from many people.

First, I would like to thank my supervisors, Prof. Michel Perez (Institut National des Sciences Appliquées de Lyon, INSA de Lyon), Dr. Sophie Cazottes (Institut National des Sciences Appliquées de Lyon, INSA de Lyon) and Dr. Thomas Sourmail (ASCOMETAL CREAS) for their tireless support and guidance throughout the project.

I would like to extend my sincere gratitude to Dr. Damien Fabregue, Prof. Jean-Yves Buffiere, Dr. Eric Maire, Dr. Daniel ACEVEDO REYES and Marion Fortey for all the useful discussion about experimental and modeling work.

Additionally, I would also like to thank Florian CHARBOIS (ASCOMETAL CREAS), Florian Mercier, Annie Malchére, Thierry Douillard and all my colleagues at the laboratories MATEIS (INSA de Lyon).

I have started my life as a lonely soul in France and along the way I have made many friends, who eventually filled the voids. Among them, I would like to specially remember Dr. Roberto Gomes de Agular Veiga, who helped me personally and also academically during the time he stayed in MATEIS.

I would like to give my special thanks to ASCOMETAL for financing this project and also giving me the opportunity to be a part of their scientific endeavor. 
Cette thèse est accessible à l'adresse : http://theses.insa-lyon.fr/publication/2013ISAL0094/these.pdf () [M.A. Razzak], [2013], INSA de Lyon, tous droits réservés 


\begin{abstract}
The objective of this thesis is to further understand austenite $(\gamma)$ Abnormal Grain Growth (AGG) phenomenon in relation with precipitation state. This study is confined to the low alloy steel material group. Abnormal grain growth is addressed from both experimental and numerical modeling points of view. The experimental techniques are consisting of determining the prior austenite grain size distribution along with the precipitate volume fraction and size distribution in different heat treated states. A time and temperature dependent grain growth behavior and precipitation state relation is drawn from the experimental data. A two-step modeling technique is adopted in this study: abnormal/normal grain growth modeling and precipitation modeling. The abnormal/normal grain growth modeling is done using a simplified analytical model where grain growth is assumed to be driven by the decrease in interfacial energy. The grain boundary energy is assumed to be isotropic and conventional Zener pinning with corner pinning by precipitates are considered as the boundary movement retarding force. The precipitation model is based on Classical Nucleation and Growth Theories (CNGTs). Homogeneous precipitate nucleation and growth assumption gave good prediction of volume fraction and mean radius in comparison with the experimental results. Heterogeneous precipitate growth is also studied and it is observed that heterogeneous precipitate growth plays an important role in precipitation kinetics when the mean austenite grain size is less than $3 \mu \mathrm{m}$. Two coupled approaches: (1) Soft coupling (2) Dynamic coupling of the abnormal grain growth and the precipitation models shed light on different physical parameters controlling the grain growth condition in a particular heat treated state. The dynamic coupled modeling enabled us to paint a comprehensive timetemperature mechanism map of the grain growth conditions. It is found that AGG in austenitic state depends strongly on the initial grain size distribution and the precipitation state. Numerical modeling and also experimental results showed that the precipitation state evolution (increasing or decreasing volume fraction) impact normal/abnormal grain growth more than the final state obtained at the end of a thermal treat-
\end{abstract}


ment. Plausible explanations in relation with the mean austenite grain size and the precipitation state are derived for the AGG phenomenon from the present work. Understanding obtained from the present work, can be utilized in designing optimum thermal treatment and alloy composition, effective in retarding AGG in austenitic state. 
Cette thèse est accessible à l'adresse : http://theses.insa-lyon.fr/publication/2013ISAL0094/these.pdf () [M.A. Razzak], [2013], INSA de Lyon, tous droits réservés 


\section{Contents}

Contents

1 Introduction 1

2 Abnormal grain growth (AGG) model 6

2.1 General introduction ................ 6

2.2 Mathematical derivation . . . . . . . . . . . . 8

2.2.1 Model assumptions . . . . . . . . . . . . . . 8

2.2.2 Abnormal and normal grain growth pressure expressions . . 9

2.3 Mechanism map with Zener pinning . . . . . . . . . . . . 11

2.4 Zener and corner pinning by second phase particles . . . . . . . . 13

2.5 AGG condition . . . . . . . . . . . . . . 16

2.6 Effects of precipitate size distribution on pinning . . . . . . . . 17

2.7 Effects of precipitate size distribution on AGG . . . . . . . . . 22

2.8 Effects of mean grain size on abnormal grain growth condition . . . 23

2.9 Application .......................... 25

2.9.1 Materials and heat treatment ............ 25

2.9.2 Results and discussion . . . . . . . . . . . . 26

2.9.2.1 Experimental observation . . . . . . . . 26

2.9.2.2 Calculation procedure .......... 26

2.9.2.3 Model prediction . . . . . . . . . . 31

2.10 Conclusions from this chapter . . . . . . . . . . . . . 33

3 Precipitation modeling $\quad 34$

3.1 Model hypothesis . . . . . . . . . . . . . . . 35

3.2 Thermodynamic equilibrium . . . . . . . . . . . . 35

3.3 Nucleation . . . . . . . . . . . . . . . . . 36

3.4 Gibbs-Thomson effects . . . . . . . . . . . . . . 38

3.5 Precipitate growth or dissolution . . . . . . . . . . . . 39

3.5.1 Growth or dissolution: Precipitate in the matrix . . . . . . 39

3.5.2 Growth or dissolution: Precipitate on grain boundary . . . . 40 
3.5.2.1 Number of precipitate lying on the grain boundary

3.5.3 Comparison between grain boundary and bulk precipitate growth or dissolution rate . . . . . . . . . . . . 45

3.6 Mass balance . . . . . . . . . . . . . . . . . 47

3.7 Implementation . . . . . . . . . . . . . . . . . . 48

3.8 Effects of model parameters . . . . . . . . . . . . 50

3.8.1 Effects of solubility product . . . . . . . . . . 50

3.8.2 Effects of precipitate interfacial energy . . . . . . . . 52

3.8.3 Effects of diffusion coefficient . . . . . . . . . . . 52

3.8.4 Effects of mean austenite grain size $\left(D_{n}\right)$ on precipitate growth 52

3.9 Model Applications . . . . . . . . . . . . . . . . . 54

3.9.1 Materials and heat treatment . . . . . . . . . . . 54

3.9 .2 Precipitate solubility products . . . . . . . . . 57

3.9.2.1 AlN solubility . . . . . . . . . . 57

3.9.2.2 NbC solubility . . . . . . . . . . . . 57

3.9.3 Precipitate size distribution . . . . . . . . . . . . 59

3.9.4 Model parameters . . . . . . . . . . . . . . . . 60

3.9.5 Precipitate volume fraction evolution . . . . . . . . . 62

3.9.5.1 Industrial alloy: steel-A . . . . . . . . . 62

3.9.5.2 Industrial alloy: steel-B . . . . . . . . . . . 64

3.9.6 Precipitate mean radius and size distribution evolution . . . 65

3.9.6.1 Industrial alloy: steel-A . . . . . . . . . . 66

3.9.6.2 Industrial alloy: steel-B . . . . . . . . . . 69

3.10 Conclusions from this chapter . . . . . . . . . . . . 69

4 Soft coupled modeling $\quad 70$

4.1 Model assumptions . . . . . . . . . . . . . . . . . . 72

4.2 Modeling strategy . . . . . . . . . . . . . . . . . 72

4.2.1 Conditions for abnormal grain growth . . . . . . . . 75

4.3 Implications of soft coupled model . . . . . . . . . . . . . . 76

4.3.1 Abnormal grain growth in presence of precipitate coarsening 77

4.3.2 Abnormal grain growth in presence of precipitate nucleation 78

4.3.3 Abnormal grain growth in presence of dissolving precipitate 81

4.4 Application to low alloy steel . . . . . . . . . . . . . . . . . . 81

4.4.1 Industrial alloy: steel-A . . . . . . . . . . . . . . 81

4.4.1.1 Composition and heat treatment . . . . . . 81

4.4.1.2 Precipitation state and grain size evolution . . . . 83

4.4.1.3 Model prediction . . . . . . . . . . . . . . 92

4.4 .2 Industrial alloy: steel-B . . . . . . . . . . . . . . . 93 
4.4.2.1 Composition and heat treatment ....... . 93

4.4.2.2 Precipitation state and grain size evolution . . . . 95

4.4.2.3 Model prediction . . . . . . . . . . . . . 97

4.5 Conclusions from this chapter . . . . . . . . . . . . 98

5 Dynamic coupled modeling $\quad 100$

5.1 Model assumptions . . . . . . . . . . . . . . . 102

5.2 Modeling strategy . . . . . . . . . . . . . . . . 102

5.2.1 Conditions for abnormal grain growth . . . . . . . . . . 104

5.3 Implications of dynamic coupled model . . . . . . . . . . . . . 106

5.3.1 Abnormal grain growth in presence of stable precipitation state106

5.3.2 Abnormal grain growth in presence of precipitate coarsening 108

5.3.3 Abnormal grain growth in presence of precipitate nucleation 110

5.3.4 Abnormal grain growth in presence on dissolving precipitate 112

5.4 Application to low alloy steel . . . . . . . . . . . . . 113

5.4 .1 Industrial alloy: steel-A . . . . . . . . . . . . . . 114

5.4.1.1 Composition and heat treatment . . . . . . . 114

5.4.1.2 Precipitation state and grain size evolution . . . . 115

5.4.1.3 Effects of heating rates on grain growth . . . . . 115

5.4.1.4 Model prediction . . . . . . . . . . . 119

5.4.1.5 Time-Temperature mechanism map of grain growth 125

5.4 .2 Industrial alloy: steel-B . . . . . . . . . . . 126

5.4.2.1 Composition and heat treatment . . . . . . 126

5.4.2.2 Precipitation state and grain size evolution . . . . . 127

5.4.2.3 Model prediction . . . . . . . . . . 127

5.4.2.4 Time-Temperature mechanism map of grain growth 130

5.4.3 Comparison between industrial alloys mechanism maps . . . 131

5.5 Conclusions from this chapter . . . . . . . . . . . . . 132

6 Conclusions and Perspectives $\quad 134$

$\begin{array}{lr}\text { A Experimental techniques } & 138\end{array}$

B A Monte-Carlo study of the compact efficiency factor $\mu \quad 142$

$\begin{array}{ll}\text { Bibliography } & 145\end{array}$ 
Cette thèse est accessible à l'adresse : http://theses.insa-lyon.fr/publication/2013ISAL0094/these.pdf () [M.A. Razzak], [2013], INSA de Lyon, tous droits réservés 


\section{Chapter 1}

\section{Introduction}

\section{Context:}

ASCOMETAL produces specialized steels for the automotive, petroleum and many other industrial applications. The specialized steels produced by ASCOMETAL demand reliability in conjunction with high performance. Satisfying the customer's demand and compete the market with progressive improvements are the most important goals. To achieve these goals, scientific understanding of the materials microstructure in relation with processing parameters are eminent. In the realm of superior mechanical, machinability, and lower rejection rate, the most demanding dimension are uniformity of the microstructure.

Automotive components such as gears, camshafts, etc require surface hardness to resist fatigue failure. Common practice for achieving surface harness is carburization at high temperature $\left(>A C_{3}\right)$. In order to control the austenite grain coarsening during high temperature carburization, precipitating elements: $\mathrm{Al}, \mathrm{Nb}$, $\mathrm{Ti}$, etc are sometimes added. Precipitating elements combine with $\mathrm{C}$ and $\mathrm{N}$ to form precipitates: $\mathrm{AlN}, \mathrm{NbC}, \mathrm{NbCN}$, etc and retard the grain boundary movement by pinning. It has been observed over the past experience of ASCOMETAL that precipitates intended for grain growth control, can induce an unexpected grain growth. The common feature of this unwanted grain growth is the presence of few extremely large grains in comparison with the mean sized grains in the matrix. This is regularly denoted as Abnormal Grain Growth (AGG). On the other hand, Normal Grain Growth (NGG) is defined as the quasi-stationary growth of whole grain size distribution.

An unexpected occurrence of AGG offers difficulties in designing heat treatments: such as carburizing. Besides the difficulties in heat treatment design, it is also reported that the presence of AGG in austenitic state can deteriorate the fatigue life of a component [64]. In figure 1.1 fatigue life of three different mi- 


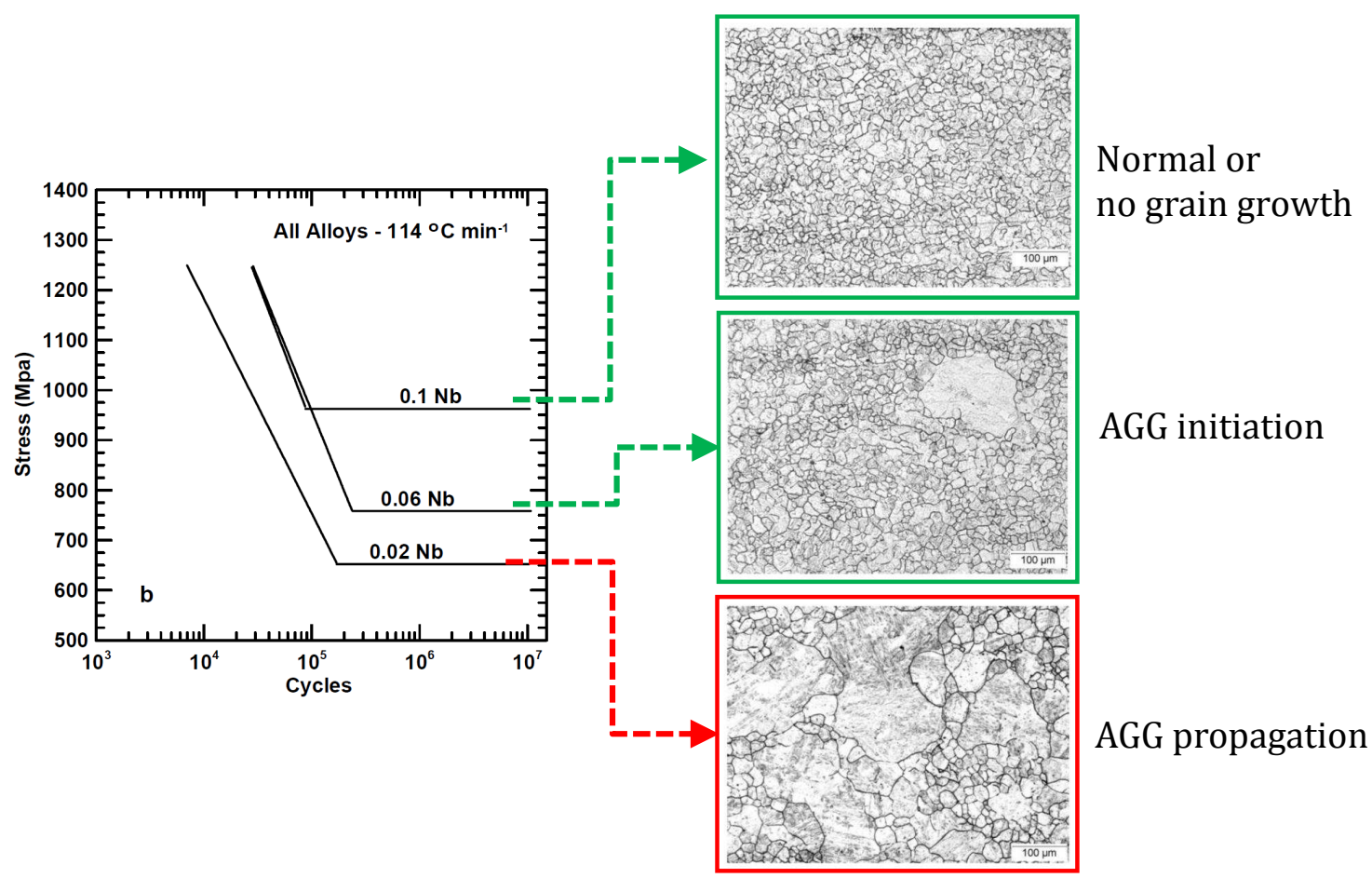

Figure 1.1: Effects of normal or no grain growth and abnormal grain growth condition on fatigue life [64].

crostructure is presented [64], where initiation and propagation of AGG lowered the fatigue life significantly. Negative effects of AGG on fatigue life demand its proper understanding in terms of initiation and propagation.

Over the past decade or so, efforts are invested to understand and prevent AGG in steel. Despite of all these efforts, AGG is little understood from the context of material chemistry and processing parameters. There are still debates about the root cause of AGG initiation. In the present project, efforts are given to extend the present understanding about AGG from the context of precipitation and processing parameters. Deterministic and probabilistic factors promoting AGG are investigated with the precipitate pinning force evolution with time and temperature. Attempts are taken to propose materials properties and processing parameters with the potential to prevent AGG in the low alloy steels.

\section{PhD project structure:}

This $\mathrm{PhD}$ project is a continuation of previous two $\mathrm{PhD}$ projects conducted under ASCOMETAL CREAS in the MATEIS laboratory. D. ACEVEDO(2007) [3] focused on the precipitate characterization during austenitization of the low alloy 


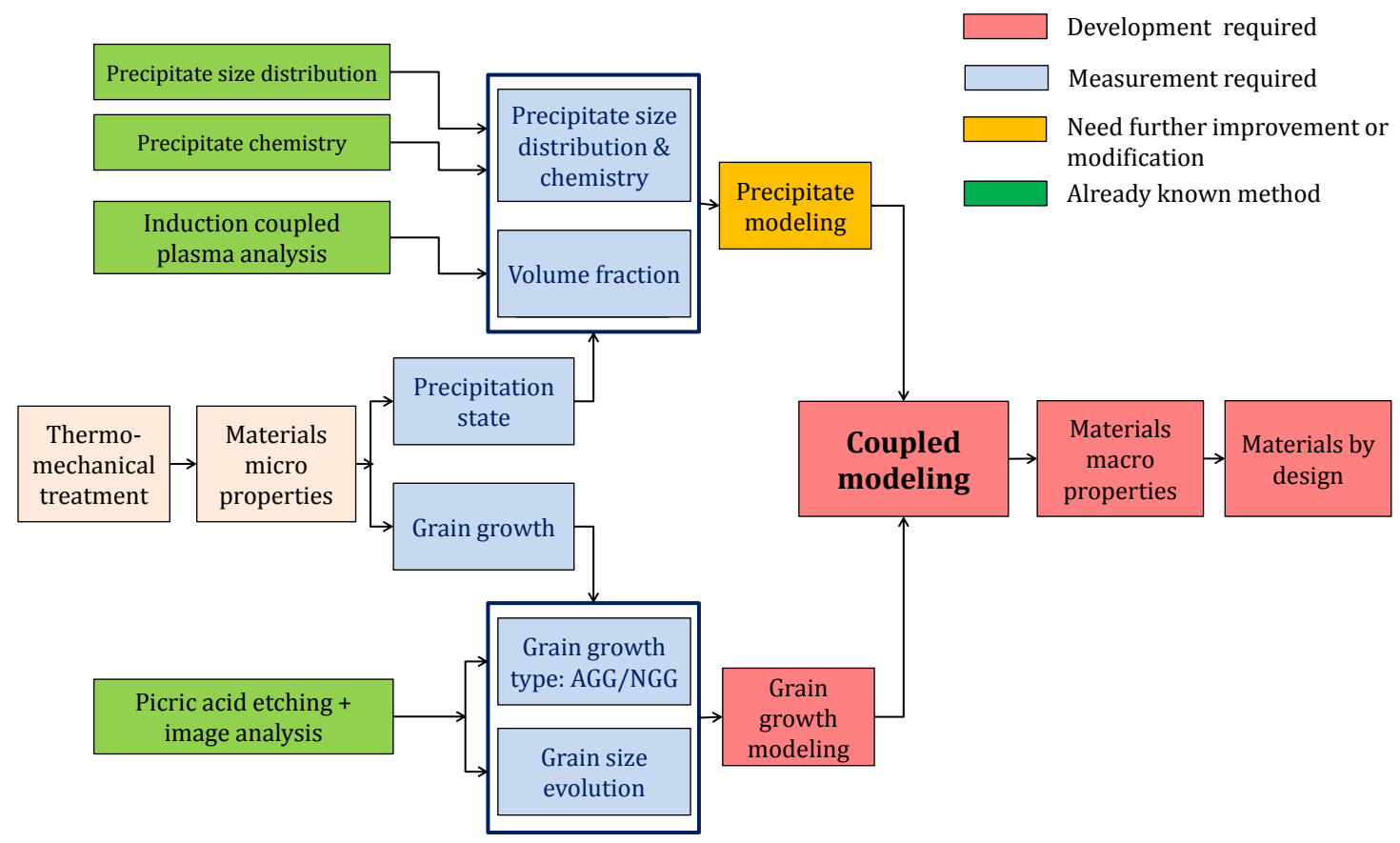

Figure 1.2: A schematic representation of the project structure.

steels. He developed a robust method of precipitation modeling (PreciSo) capable of predicting precipitation state using the Classical Nucleation and Growth Theories (CNGTs). This model considered dilute solid solution approximation with homogeneous precipitation conditions. Following his work, C. Leguen(2010) [40] investigated the austenite grain growth phenomenon in the low alloy steels in relation with precipitation state. In order to predict onset of Abnormal Grain Growth (AGG) in the austenite state, she proposed a criterion for AGG initiation. The proposed criterion incorporated the precipitate size distribution in pinning when both conventional Zener pinning with corner point pinning take place. The present work is initiated to progress the previous works understanding about austenite grain growth behavior in relation with precipitation state.

The objective of this $\mathrm{PhD}$ project is to further understand the relationship between precipitation and austenite grain growth phenomenon in low alloy steels. For this reason, it is necessary to experimentally determine the precipitation state in different heat treated states and also the grain size distribution. Grain size distribution analysis shows grain growth type in a particular heat treated state together with the grain size evolution. Experimental measurement of micro scale properties such as: grain size and precipitation state can then be utilized to predict macro scale physical properties. Numerical modeling is usually done to minimize the tedious and time consuming experimental work. In this particular work, emphasis is given to develop a modeling scheme capable of simultaneously predicting the precipitation state and the grain growth condition at different times and tempera- 
tures.

The project organization is illustrated in the figure 1.2. The known and unknown methods are outlined using different color codes. Following the schematics, the tasks planned in this project can be summarized as follows:

1. Grain growth state needs to be identified by the grain size distribution measurement.

2. Precipitation state characterization is required in terms of both volume fraction and size distribution.

3. An abnormal grain growth modeling concept will be developed to predict the grain growth condition in different precipitation states.

4. A precipitation modeling is required to bridge the effects of heat treatment on precipitation state. This modeling technique will enable us to predict the precipitation state and reduce the required number of experiments.

5. The abnormal grain growth model and precipitation model will be coupled together to predict the grain growth condition in relation with precipitation state in different heat treated states.

\section{Manuscript organization:}

The objective of this project is to understand and devise a methodology to predict prior austenite grain growth behavior in the presence of second phase particles. In order to achieve the main objective, several tasks are undertaken which can be recognized with individual characteristics. These tasks are grouped together to form a particular chapter in the present manuscript. A short description of each chapter is presented in the following:

In chapter 2, a simplified Abnormal Grain Growth (AGG) model is presented. The mathematical derivation of abnormal and normal grain growth pressure is presented where the grain growth is driven by the decrease in interfacial energy. It is assumed that grain boundary pinning is caused by second phase particles. Depending on particles location, two kinds of pinning can occur in the microstructure: (1) conventional Zener pinning and (2) corner pinning. A detailed model application procedure for a static precipitation state is explained in this chapter. Finally, the model is applied to a model alloy (FeVNbCN) to verify its effectiveness in prediction AGG and Normal or No Grain Growth (NGG) in low allow steel.

In chapter 3, a precipitation model based on the Classical Nucleation and Growth Theories (CNGTs) is presented. Homogeneous and heterogeneous pre- 
cipitate growth are investigated to understand the effects of both mechanism on the precipitation kinetics. The aim is to find out if precipitates lying at the grain boundaries and the corner points experience accelerated growth or dissolution, the impact on precipitation state evolution. This investigation is rather important as during the grain growth, precipitates that reside at the grain boundaries only contribute to the pinning. Transmission Electron Microscopy (TEM) is performed on two industrial alloys: (1) steel-A and (2) steel-B, to characterize the precipitate size distribution. Induction Coupled Plasma (ICP) analysis is performed to quantify the precipitate volume fraction in the different heat treated states. Finally, the precipitation model is applied and model results are tested in comparison with the experimental results obtained from two industrial alloys.

A coupled modeling between the precipitation and abnormal grain growth models is presented in chapter 4 . This particular modeling approach is termed as the 'Soft Coupled Modeling (SCM)'. In order to reduce complexity in calculation in the $\mathrm{SCM}$, mean grain size is assumed to be constant throughout the simulation time. So, in a static mean grain size system, only the precipitation state evolution is determining the grain growth conditions (AGG or NGG) in the microstructure. The SCM is applied to two industrial alloys and the results are compared with experimental observations. Agreements and disagreements between model predictions and experimental observations are explained in order to deliminate the modeling scheme's strengths and limitations.

In chapter 5, a sophistication in coupled modeling is introduced by calculating the evolution of mean austenitic grain size with the precipitation state evolution with time at a particular temperature. This coupled modeling approach is defined as the 'Dynamic Coupled Modeling (DCM)'. The DCM depicts a real situation in the material microstructure, where both mean grain size and precipitation state evolve simultaneously. The DCM is applied to two industrial alloys and modeling results are compared with the experimental ones. The DCM enabled us to draw a time-temperature mechanism map of the grain growth condition of the two industrial alloys. The experimental observations are superimposed on mechanism maps to deliminate capabilities and limitations of the DCM.

In the final chapter, a collection of all the major conclusions derived from the project is presented. In order for the possible extension of the present work, future possibilities are also discussed. 
Cette thèse est accessible à l'adresse : http://theses.insa-lyon.fr/publication/2013ISAL0094/these.pdf () [M.A. Razzak], [2013], INSA de Lyon, tous droits réservés 


\section{Chapter 2}

\section{Abnormal grain growth (AGG) model}

In this chapter, attempts are made to address the abnormal grain growth phenomenon from a numerical modeling point of view. A model is proposed considering three dimensional grain structure using analytical expressions. Stability of a comparatively larger grain surrounded by smaller ones is predicted using the balance between grain growth and pinning pressure. Conventional Zener pinning and also corner pinning are introduced as grain growth retardant where the whole precipitate size distribution is taken into account. The proposed model overcomes some of the previous model's shortcomings by introducing precipitate's size distribution dependent pinning instead of precipitate mean radius (mono disperse distribution) consideration.

\subsection{General introduction}

Normal Grain Growth (NGG) in materials is defined when the grain size distribution remains quasi-stationary during coarsening phenomenon [5]. In a more simplistic term, NGG can be defined when the mean grain diameter evolves but the grain size distribution shape remains unchanged. On the other hand, Abnormal Grain Growth (AGG) is defined as the rapid growth of few number of grains in comparison with the others. A microstructure subjected to AGG has the characteristics of having very large grains surrounded by relatively smaller ones [21].

The causes of AGG are not yet very well understood. Some authors claim that AGG is due to the variable grain boundary mobility (e.g. induced by texture) [56]. Whereas others cite the second phase particles (i.e. precipitates) as the cause of AGG [5, 9]. Anisotropy in the grain boundary mobility can be a dominant reason for AGG where texture induced by recrystallization occurs. Considering low alloy 
steel, where recrystallization induced texture is seldom found, precipitate controlled AGG is more likely to occur [30]. Besides precipitate induced pinning, AGG is also associated with solute drag effects [37]. It is reported that the grain-growth mode can be changed depending on the solute diffusivity in the grain boundary where the initial average grain size plays an important role. From a modeling point of view, Monte Carlo and phase field approaches take into account the commonly assumed anisotropic grain boundary energy [57,33]. The anisotropic grain boundary energy allows particular grains in a heterogeneous microstructure to grow preferably faster than the others. Thus comparatively rapid growth of some grains attain abnormality in the microstructure.

There are numbers of authors who have deduced the parabolic grain growth relationship for mean grain size in the case of normal grain growth (see the pioneer paper of Hillert [24] and the review of Gladman [21]). Subsequently, these models were extended to predict and explain AGG in materials. M. Hillert addressed the "abnormal" or "discontinuous" grain growth phenomenon as a defect model [24]. In this model, the growing and the shrinking grains are determined by the number of neighbors it has.

Zener [71] first proposed that during grain growth, second phase particles present in the matrix, exert back pressure during the boundary movement. This back pressure is commonly known as the Zener Pinning. Most of the previous works explained AGG initiation with local unpinning of larger grains [24, 5, 20]. The higher growth pressure of larger grains overcome the pinning and subsequently grow in an abnormal manner. Bréchet et al. [9] introduced corner pinning that led to a grain size dependent pinning, which may contribute to AGG initiation. According to their hypothesis, corner pinning is more efficient in pinning grain boundaries than conventional Zener pinning, leading to a net pinning force that decreases with increasing grain size, which is itself the cause of AGG instability. Here it should be mentioned that precipitate lying at the triple or quadruple junctions contributes to corner pinning. A more detailed explanation of Zener and corner pinning given in the section 2.4. This model has been recently extended to account for the whole precipitate size distribution and give an explanation for AGG initiation [40] and propagation [53].

Andersen et al. [5] proposed a 2D analytical model in which an abnormal grain is surrounded by an array of hexagonal normal grains. Andersen proposed a grain growth mechanism map, in which AGG occurs when (i) smaller grains are pinned and (ii) an abnormal grain is large enough in comparison with the normal grains.

In this chapter, we will propose a criterion for AGG propagation in the presence of second phase precipitates. Based on a 3D geometrical consideration, a simple model to predict the stability of an existing AGG situation will be presented. Grain 

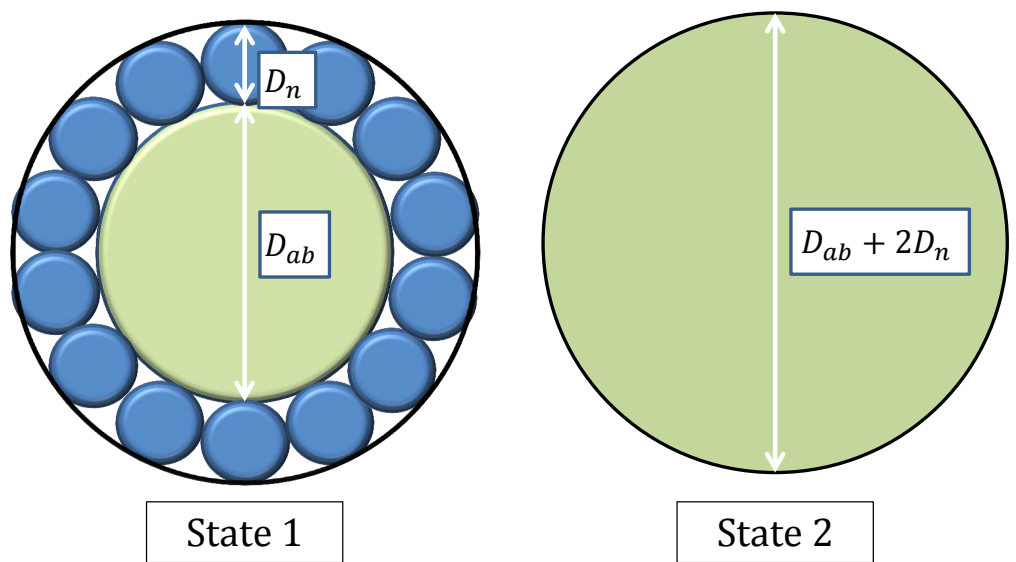

Figure 2.1: A schematic representation of an abnormal grain of diameter $D_{a b}$ surrounded by normal grains of diameter, $D_{n}$. The abnormal grain is growing from state (1) to state (2) by consuming all the surrounding normal grains and increasing its diameter by $2 D_{n}$.

boundary pinning is assumed to occur by both the classical Zener mechanism and corner point pinning. This model aims at predicting the conditions, in terms of precipitation state, for which AGG may occur.

\subsection{Mathematical derivation}

\subsubsection{Model assumptions}

The following assumptions are made in the AGG model:

1. The grains are assumed to be of high angle type and isotropic. So, the effects of anisotropic grain boundary energy and mobility are avoided.

2. Solute drag force in the system is assumed to be comparatively lower than precipitate induced pinning. So, solute drag has a negligible influence in retarding the grain boundary movement. For this reason, solute drag is not considered in the present model.

3. The precipitates are finely distributed in the microstructure. Fluctuation in the pinning force due to solute segregation is not considered in the system.

Although there are some model assumptions introduced, the fundamental mechanism of grain growth remain unchanged. Moreover, the assumptions allowed us to formulate an AGG model with a reduced number of variables and fitting parameters. 


\subsubsection{Abnormal and normal grain growth pressure expres- sions}

The theory for grain growth is based on the decrease in grain boundary interfacial energy being the driving force. In the case of AGG, the driving pressure of the abnormal grain is larger than the neighboring grains and finally consumes them. In this study, grains are represented as sphere (see Figure 2.1). A large grain of diameter $D_{a b}$ (the abnormal grain) is surrounded by smaller (normal) grains of diameter $D_{n}$ (state (1). The abnormal grain can increase its diameter at the expense of surrounding normal grains and attain a new diameter of $\left(D_{a b}+2 D_{n}\right)$ (state (2)).

The total work $\delta W=E_{1}-E_{2}$ required to move the system from state (1) to state (2) determines the driving pressure $P_{a b}$ of abnormal grain to grow (as shown in figure 2.1), such as $\delta W=P_{a b} \delta V$. Here, $\delta V$ is the volume change of abnormal grain from state (1) to state (2). It is given by: $\delta V=\lambda_{a b} D_{a b}^{2} \delta D_{a b} / 2$, where $\lambda_{a b}$ is a geometric factor $\left(\lambda_{a b}=\pi\right.$ for a sphere).

The total number of normal grains $n_{g}$, surrounding the abnormal grain depends on $D_{n}$ and $D_{a b}$. It can be estimated as the ratio between the total available surface around the abnormal grain over projected surface of the normal grains:

$$
n_{g}=\rho \frac{\lambda_{a b}\left(D_{a b}+D_{n}\right)^{2}}{\lambda_{p} \lambda_{n} D_{n}^{2}}=4 \mu \frac{\lambda_{a b}}{\lambda_{n}} \frac{\left(D_{n}+D_{a b}\right)^{2}}{D_{n}^{2}}
$$

Here, $\rho$ is the compact efficiency factor, $\lambda_{n}$ is a geometric factor $\left(\lambda_{n}=\pi\right.$ for a sphere), $\lambda_{p}$ is the ratio between projected surface over actual surface of the normal grains $\left(\lambda_{p}=1 / 4\right.$ for a plane) and $\mu=\rho /\left(4 \lambda_{p}\right)$. A Monte-Carlo (MC) study of the possible value of $\mu$ in a normal grain size distribution is given in appendix B. It is found from the MC study that $\mu$ has a value ranging between 0.54 and 0.56 . The total interfacial energy of state (1) (see figure 2.1) is given by:

$$
E_{1}=\frac{\gamma_{g}}{2}\left[\lambda_{a b} D_{a b}^{2}+n_{g} \lambda_{n} D_{n}^{2}\right]=\frac{\gamma_{g} \lambda_{a b}}{2}\left[D_{a b}^{2}+4 \mu\left(D_{a b}+D_{n}\right)^{2}\right]
$$

Here, $\gamma_{g}$ is the grain boundary interfacial energy. Similarly, total interfacial energy of the state (2) (see figure 2.1) is:

$$
E_{2}=\frac{\gamma_{g} \lambda_{a b}}{2}\left[D_{a b}+2 D_{n}\right]^{2}
$$

The driving pressure of an abnormal grain growth is determined by the work $\delta W$ done to increase its volume by $\delta V$ :

$$
P_{D_{a b}}=\frac{\delta W}{\delta V}=\frac{E_{1}-E_{2}}{\delta D_{a b}} \frac{\delta D_{a b}}{\delta V}=\frac{E_{1}-E_{2}}{2 D_{n}} \frac{2}{\lambda_{a b} D_{a b}^{2}}
$$


The abnormal grain driving pressure can be expressed as:

$$
P_{D_{a b}}=\frac{2 \gamma_{g}}{D_{n}}\left[\mu-(1-\mu)\left(\frac{D_{n}}{D_{a b}}\right)^{2}+(2 \mu-1) \frac{D_{n}}{D_{a b}}\right]
$$

The grain coarsening phenomenon implies that grain boundaries move through the matrix. In the presence of second phase particles, there is always a possibility that any grain boundary will eventually meet second phase particles and experience pinning.

Zener showed that second phase particles produce a back stress which can hinder the boundary movement. A particle of radius $r$ exerts a force $f_{z}=k_{s} r \gamma_{g}$ on grain boundary, where $k_{s}$ is a geometrical factor, having a value of $\pi$ for spherical particles. Assuming a random distribution, the number density of second phase particles $(N)$ is related to the precipitate volume fraction $(f)$ by $f=N k_{v} \bar{r}^{3}$, where $k_{v}$ is another geometrical factor, having a value of $4 \pi / 3$ for spherical particles and $\bar{r}$ is the mean radius of precipitates. Finally, the number $n_{s}$ of particles interacting with $1 \mathrm{~m}^{2}$ of grain boundary is $n_{s}=2 \bar{r} N$ and the pinning pressure can be expressed as:

$$
\bar{P}_{Z}=n_{s} f_{z}=\frac{2 k_{s} \gamma_{g} f}{k_{v} \bar{r}}
$$

Now, if we consider a distribution of second phase particles, the precipitate size distribution is described by $n$ number of classes with radii $r_{i}$ populated with $n_{i}$ precipitates per unit volume. From the knowledge of precipitate size distribution (i.e. the function $n_{i}\left(r_{i}\right)$ ), the total pinning pressure is simply a sum over pinning pressure from each precipitate size class $(i)$ :

$$
P_{Z}=\sum_{i}^{n} 2 r_{i} n_{i} k_{s} r_{i} \gamma_{g}=2 k_{s} \gamma_{g} N_{r}, \text { where } N_{r}=\sum_{i}^{n} n_{i} r_{i}^{2}
$$

Note that classical Zener pinning pressure does not depend on the grain size distribution. However, driving pressure of normal grain growth does depend on the grain size. It is usually given by $P_{D_{n}}=\lambda \gamma_{g} / D_{n}$, where $\lambda$ is a geometrical constant. Zener and Gladmann [21] proposed a value of 4 for $\lambda$. More recently, based on model results, Rios [54] proved a more accurate expression to be 8 times smaller than the Zener pinning. The grains are suppose to grow until they reach a limiting size of $D_{n}^{l}$, for which the driving pressure equals the pinning pressure, leading to:

$$
P_{Z}=\frac{\lambda \gamma_{g}}{D_{n}^{l}} \text { with } D_{n}^{l}=\frac{\lambda k_{v}}{2 k_{s} N_{r}}
$$

The limiting grain size $D_{n}^{l}$ is often used as a parameter to quantify pinning efficiency of a second phase particle distribution. 
Considering Zener pinning pressure over all the grains, a simple expression for abnormal grain growth rate $[30,20,24]$ can be written as:

$$
\frac{d D_{a b}}{d t}=M\left(P_{D_{a b}}-P_{Z}\right)
$$

Here, $M=M_{0} \exp \left(-\frac{Q}{R T}\right) . M$ is mobility of the grain boundary, $Q$ is activation energy, $R$ is molar gas constant, $T$ is temperature and $M_{0}$ is a pre-exponential factor. The same expression holds for normal grain growth rate [30, 20, 24]:

$$
\frac{d D_{n}}{d t}=M\left(P_{D_{n}}-P_{Z}\right)
$$

Two conditions of AGG are classically stated as follows:

$$
\frac{d D_{a b}}{d t}>0 \text { and } \frac{d}{d t}\left(\frac{D_{a b}}{D_{n}}\right)>0
$$

Equation 2.11 states that in order for AGG to occur, first of all larger grains $\left(>D_{n}\right)$ should have the ability to growth $\left(d D_{a b} / d t>0\right)$. Besides that, larger grains in the system should have suitable conditions to grow comparatively faster than the mean sized grains $\left(d / d t\left(D_{a b} / D_{n}\right)>0\right)$. Only fulfilling both conditions will allow a system to experience AGG phenomenon.

From equations 2.5, 2.8, 2.9 and 2.10, and assuming $x=D_{n} / D_{n}^{l}$ and $y=$ $D_{a b} / D_{n}$, the two conditions for AGG can be expressed as:

$$
\begin{gathered}
\mu-(1-\mu) y^{-2}+(2 \mu-1) y^{-1}-x \lambda / 2>0 \\
\mu-(1-\mu) y^{-2}+(2 \mu-1) y^{-1}-\lambda / 2[x+y(1-x)]>0
\end{gathered}
$$

Equations 2.12 and 2.13 can be solved numerically. The results obtained over a range of $x$ and $y$ yield tentative conditions of NGG and AGG.

\subsection{Mechanism map with Zener pinning}

Solutions of the equations 2.12 and 2.13 are plotted in figure 2.2. Figure 2.2 represents a mechanism map of AGG for $\mu=0.54$ as a function of $x=D_{n} / D_{n}^{l}$ (pinning pressure) and $y=D_{a b} / D_{n}$ (initial grain size of the instability). As expected, abnormal grain growth $\left(d D_{a b} / d t\right)$ is hindered by second phase particles pinning and favored when the difference between normal and abnormal grain is large (higher driving force). However, as abnormal grain becomes larger, the relative driving force of normal grains to grow also increases. The relative growth of abnormal grains $\left(d\left(D_{a b} / D_{n}\right) / d t\right)$ is then favored when (i) pinning pressure is high 


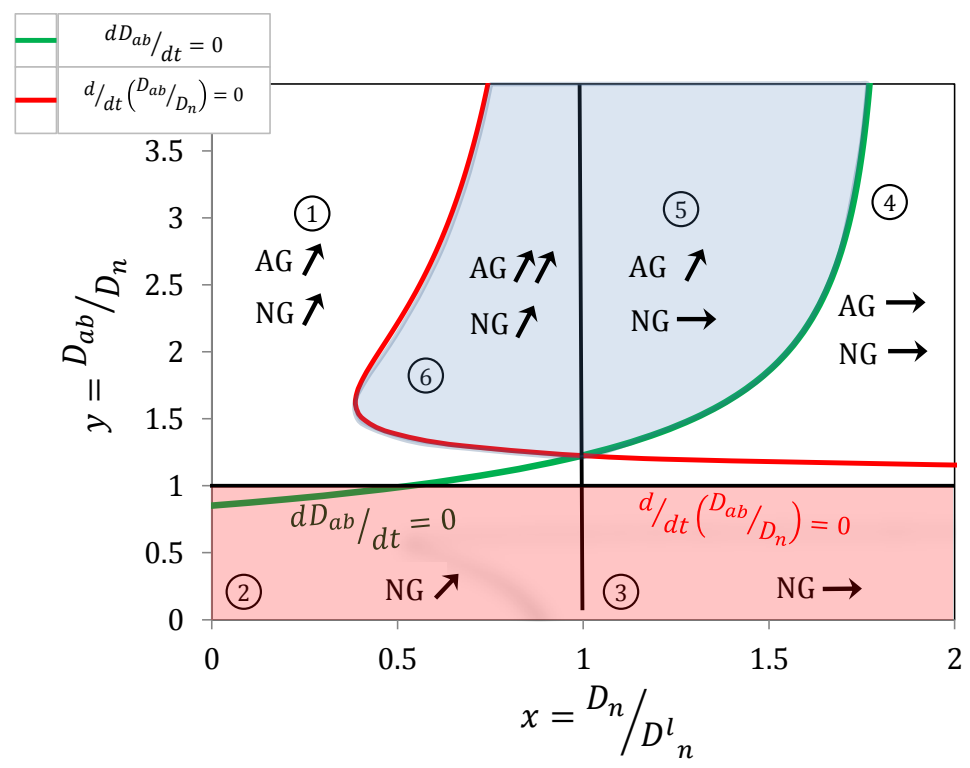

Figure 2.2: A mechanism map of AGG considering Zener pinning with $\mu=0.54$ and $\lambda=0.6$. The map exhibits 6 domains: (1): normal and abnormal grains grow in a stable manner, (2): normal grains can grow, (3): normal grains are pinned, (4): both abnormal and normal grains are pinned, (5): normal grains are pinned, whereas abnormal grain grows (unstable AGG region) and (6): normal and abnormal grain grow in an unstable manner (unstable AGG region). $y$ scale is valid above the red shaded area from $y=1$.

and (ii) size of abnormal grain relative to normal grains is moderately large. The ideal conditions of AGG seem to be the same as the ideal conditions to have small grains. In other words, the price to pay to keep a fine microstructure potentially leads to an unstable situation.

It is remarkable how similar the present mechanism map looks like, compared to Andersen's et al. [5]. In the limiting case where $D_{n} / D_{n}^{l}$ approaches unity, we found that an abnormal grain with a size advantage greater than about $1.4 D_{n}(\mu=0.54)$ to $2 D_{n}$ will continue to grow abnormally. Anderson et al. also found a value of $1.4 D_{n}$.

In figure 2.3, the effects of compact efficiency factor $(\mu)$ are shown on AGG mechanism map. An increasing value of $\mu$, increases the AGG region and shifts the AGG initiation point to the left in the map. A highly compact microstructure has a larger difference in diameter between pre-existing larger grains and smaller ones. This increases the driving energy of AGG, hence increasing its probability to occur even at higher pinning condition. In the presented model, $\mu$ is a fitting parameter. For a particular alloy, the value of $\mu$ is chosen which describes the evolution of grain growth condition in the system accurately. So, it is understandable that $\mu$ might have different values ranging between 0.54-0.56 (see appendix B), in different alloy 


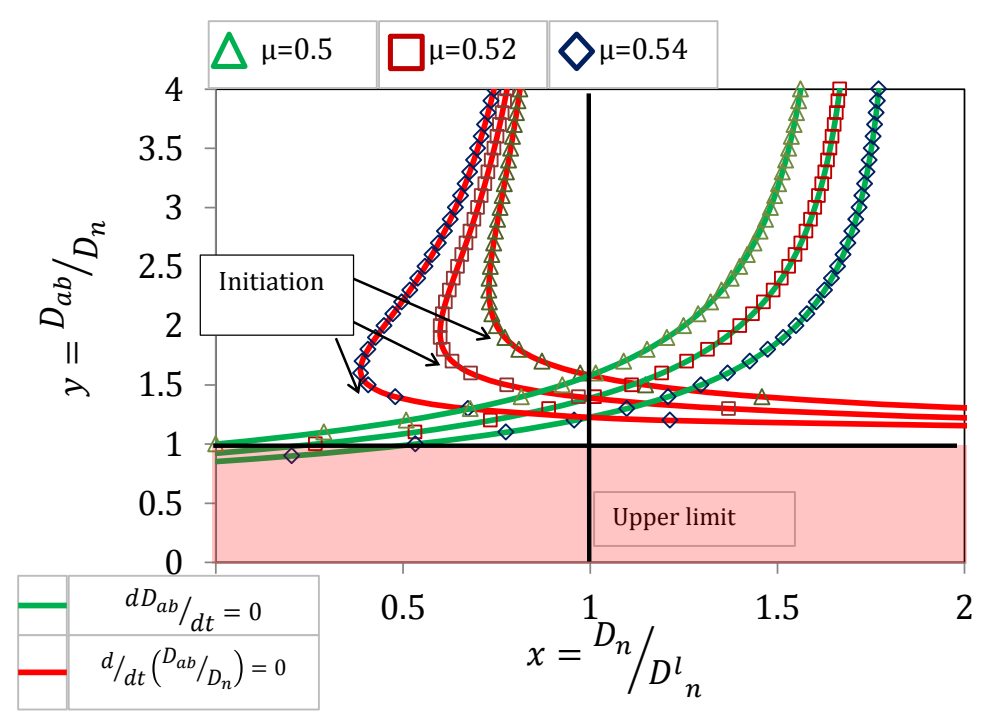

Figure 2.3: Mechanism map of AGG considering Zener pinning with $\lambda=0.6$ and $\mu=0.5,0.52$ and 0.54 . It can be observed that with an increasing value of $\mu$, enclosed AGG region also increases.

systems.

\subsection{Zener and corner pinning by second phase par- ticles}

Bréchet et al. . [9] proposed that particles lying on triple junctions or quadruple points (special location) are more effective in pinning the boundary movement than particles lying on the boundary. Their proposed corner pinning force being $f_{c}=\alpha_{0} f_{z}$, where $\alpha_{0}$ is a constant larger than 1 . They considered that quadruple points are preferential locations for precipitates: i.e. if the number of precipitates is large enough, all the corner points will be occupied, whereas remaining particles participate in classical Zener pinning. They estimated the number of quadruple points and proposed an expression for the net pinning force being the addition of corner point pinning and Zener pinning.

It has been shown [39] that in some cases, the precipitate population is poorly described by taking only average quantities, i.e. the mean radius instead of the whole distribution. In this work, Zener pinning and corner point pinning are considered taking into account the whole precipitate size distribution.

From equation 2.7, it is apparent that larger precipitates create higher pinning force. It can be assumed, austenite grain boundaries which previously got pinned on the larger particles subsequently become triple junction or corner point. The assumption that a special location is created at larger particles creates a guideline 
for further evolution of modeling concept using particles size distribution. In this model, it is assumed that larger particles occupy the special location preferentially. After filling all the special location in descending radius, rest of the precipitate classes participate in conventional Zener pinning.

In a matrix, where an abnormal grain of diameter $D_{a b}$ is surrounded by normal grains of diameter $D_{n}$, the number of corner points per unit volume is shown by expression 2.14, where $K_{0}$ is the number of corner points per grain and $K_{V}$ is a geometrical factor.

$$
N^{*}=\frac{K_{0}}{K_{V} D_{n}^{3}}[9]
$$

If we consider a mono disperse distribution (mean radius $(\bar{r})$ ) with total number of precipitate $N$, then the corner point pinning $\left(\bar{P}_{C}\right)$ can be expressed as follows:

where $N>N^{*}$

$$
\bar{P}_{C}=K_{0}\left(\alpha_{0} k_{s} \gamma_{g} \bar{r}\right) \frac{1}{K_{A} D_{n}^{2}}
$$

where $N<N^{*}$

$$
\bar{P}_{C}=\frac{N}{N^{*}} K_{0}\left(\alpha_{0} k_{s} \gamma_{g} \bar{r}\right) \frac{1}{K_{A} D_{n}^{2}}
$$

Let us consider a particles size distribution $n_{i}\left(r_{i}\right)$ with a total number of precipitates $n_{n}=\sum_{i} n_{i}$. As stated before larger particles reside in the special locations (quadruple or corner point), smaller ones participate in conventional Zener pinning. There should be a critical radius $r_{c}$ of the particle classes where partitioning between the particle classes occurs for Zener and corner point pinning. As reported by Bréchet et al.. pinning force is dependent on the number of the corner points available in the system. If $n_{n}<N^{*}$ :

$$
P_{C}=\sum_{i} \frac{n_{i}}{N^{*}} \frac{K_{0} \alpha k_{s} \gamma_{g} r_{i}}{K_{A} D_{n}^{2}}=\frac{K_{V} \alpha k_{s} \gamma_{g}}{K_{A}} D_{n} \sum_{i=1}^{n} n_{i} r_{i}
$$

If $n_{n}>N^{*}$, the total pinning force is given by $P_{C}$ and $P_{Z}$ :

$$
P_{C}=\frac{K_{V} \alpha k_{s} \gamma_{g}}{K_{A}} D_{n} \sum_{i=i^{*}}^{n} n_{i} r_{i} \text { and } P_{Z}=2 k_{s} \gamma_{g} \sum_{i=1}^{i^{*}} n_{i} r_{i}^{2}
$$

Where, $i^{*}$ is the index of the precipitate class from which the precipitates are either lying in the corner points or involved in classical Zener pinning. As the pinning force of precipitate in the corner points is $f_{C}=\alpha_{0} k_{s} \gamma_{g} r_{i}$, larger precipitates have a higher probability of lying at the corner points (corner points being created at the largest precipitate). Therefore, the smaller precipitates of class index 1 to 


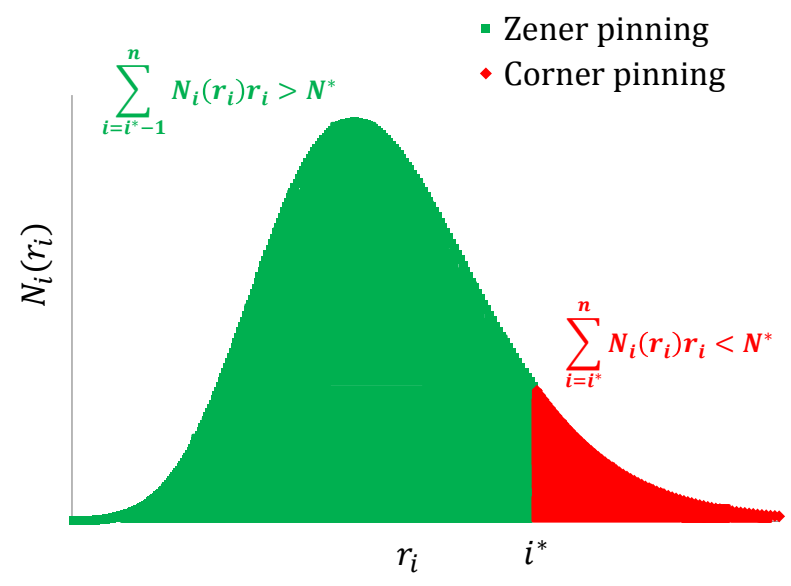

Figure 2.4: Precipitate class starting from the largest radius contributing to corner pinning (red) and rest of the classes contributing to classical Zener pinning.

$i^{*}$ are involved in classical Zener pinning whereas larger precipitates of class index $i^{*}$ to $n$ are lying in the corner points :

$$
\sum_{i=i^{*}}^{n} n_{i}<N^{*}<\sum_{i=i^{*}-1}^{n} n_{i}
$$

In figure 2.4, the expression 2.19 is represented graphically. Here, precipitate class with largest radius fill the corner points till $i^{*}$ (classes with red color) and starting from $\leq i^{*}$, rest of the classes of precipitate participate in Zener pinning.

Note that, $i^{*}$ depends on $D_{n}$ : the smaller is $D_{n}$, the larger is $N^{*}$ and consequently, the smaller is the index $i^{*}$. Considering both corner and Zener pinning simultaneously, the abnormal grain growth rate equations 2.9 and the normal grain growth rate equation 2.10 can be modified as follows:

$$
\begin{aligned}
& \frac{d D_{a b}}{d t}=M\left[P_{D_{a b}}-P_{C}-P_{Z}\right] \\
& \frac{d D_{n}}{d t}=M\left[P_{D_{n}}-P_{C}-P_{Z}\right]
\end{aligned}
$$

The second condition, $d / d t\left(D_{a b} / D_{n}\right)$ of abnormal grain growth can be expressed by combining the equation 2.20 and 2.21 as follows:

$$
\frac{d}{d t}\left(\frac{D_{a b}}{D_{n}}\right)=\frac{1}{D_{n}} \frac{d D_{a b}}{d t}-\frac{D_{a b}}{D_{n}^{2}} \frac{d D_{n}}{d t}
$$

The addition of corner pinning with Zener pinning pressure, increases the total pinning pressure in the system. The abnormal grain critical diameter is determined by equilibrium between the growth pressure and the pinning pressure. An increase in pinning pressure eventually leads to a larger critical diameter than the first case, 
where only Zener pinning is considered.

\subsection{AGG condition}

In this section, application of the AGG conditions (see expression 2.11) in identifying grain growth condition in a real microstructure is discussed. The delimitating factors of the AGG condition that determine the grain sizes in a distribution susceptible to abnormal growth is defined. The objective is to further simplify the grain growth condition representation in an evolving (with time) system (evolution of precipitation size distribution $\left(N_{i} r_{i}\right)$ and mean grain $\left.\operatorname{size}\left(D_{n}\right)\right)$.

We can recall the AGG conditions:

$$
\frac{d D_{a b}}{d t}>0 \text { and } \frac{d}{d t}\left(\frac{D_{a b}}{D_{n}}\right)>0
$$

Expression 2.22 suggest that in order for the second condition $\left(d / d t\left(D_{a b} / D_{n}\right)>\right.$ $0)$ to be true, the first condition $\left(d D_{a b} / d t>0\right)$ has to be true. So, AGG condition can be described only by the second condition. The abbreviated form of the second condition considering both Zener and corner pinning is given below:

$$
\begin{aligned}
\frac{d}{d t}\left(\frac{D_{a b}}{D_{n}}\right) & =\frac{1}{D_{n}} \frac{d D_{a b}}{d t}-\frac{D_{a b}}{D_{n}^{2}} \frac{d D_{n}}{d t} \\
& =\frac{2 \gamma_{g} M}{D_{n}^{2}}\left[\mu-(1-\mu)\left(\frac{D_{n}}{D_{a b}}\right)^{2}+(2 \mu-1) \frac{D_{n}}{D_{a b}}-\frac{\lambda D_{a b}}{2 D_{n}}\right] \\
& -\frac{M}{D_{n}}\left(\frac{D_{a b}}{D_{n}}+1\right)\left(P_{C}+P_{Z}\right)
\end{aligned}
$$

Here, $D_{a b}$ can vary between $D_{n}$ and $\infty$ and it is only true when $D_{n} / D_{n}^{l}>1$.

The second condition 2.24 expression of AGG is in quadratic form which is quite similar to reported by Mullin et al. [56] and Humphreys [30]. There are two values of $D_{a b}$ delimiting a domain within which the AGG condition is fulfilled. The smallest value of $D_{a b}$ can be denoted as $D_{a b}^{\min }$ and the largest as $D_{a b}^{\max }$ (see figure 2.5). In fact, in a no pinning condition, for $\mu=0.56$ and $\lambda=0.6$, it can be found that AGG condition is valid between, $D_{a b}^{\min } / D_{n} \approx 1.26$ and $D_{a b}^{\max } / D_{n} \approx 1.43$. In figure 2.5, $d / d t\left(D_{a b} / D_{n}\right)$ obtained for increasing pinning conditions are superimposed on a normal grain size distribution. As shown in figure $2.5, D_{a b}^{\max }$ can be within the grain size distribution and very close to the mean sized grain (1) or close but smaller than the largest grain size (2) or larger than the maximum grain size in the distribution (3). Among these three situation, only (3) bears the probability 


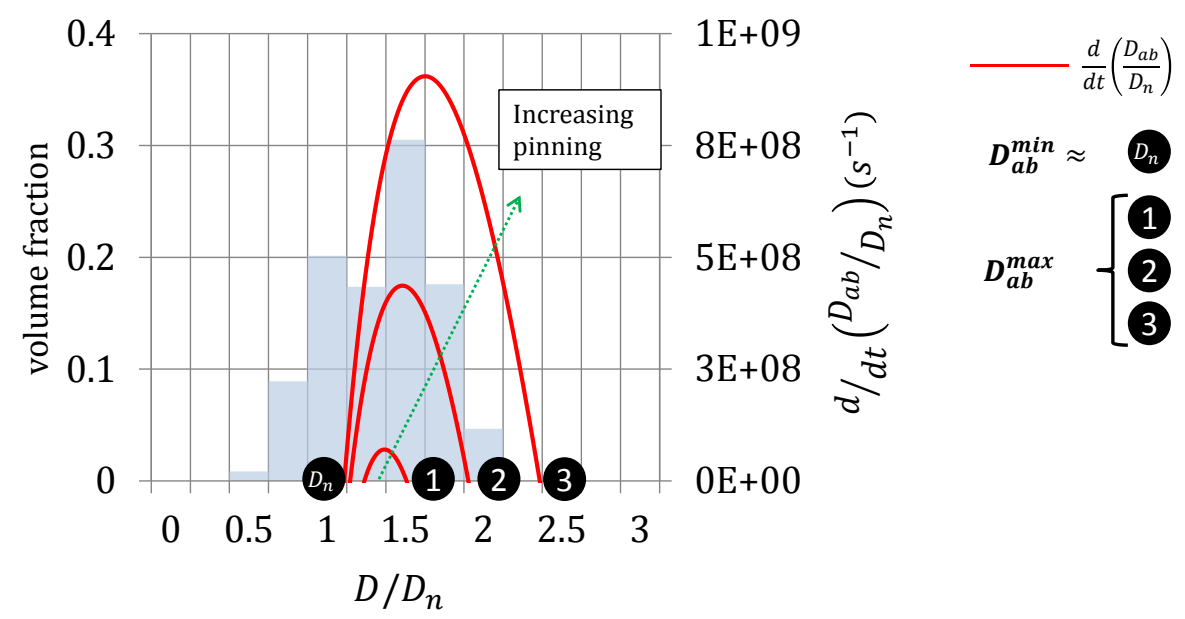

Figure 2.5: Superimposition of AGG condition $\left(d / d t\left(D_{a b} / D_{n}\right)>0\right)$ considering different pinning condition on a grain size distribution. $d / d t\left(D_{a b} / D_{n}\right)$ can range between $D_{n}$ and (1) or (2) or (3). Here, it is assumed that $M=1$.

of AGG initiation. In case (3), the largest grains in the microstructure can grow relatively faster than the mean sized ones. In cases (1) and (2) grain sizes very close to the mean sized one grow faster which in fact facilitates a quasi-stationary grain size distribution evolution (normal grain growth). From figure 2.5, it can be concluded that $D_{a b}^{\max }$ must be at least 2 times of $D_{n}$ in order for AGG to occur. So, a realistic qualitative identifier of AGG and NGG can be expressed as follows:

$$
\begin{aligned}
& D_{a b}^{\max } / D_{n}>2 \text { Probable grain growth condition: AGG } \\
& D_{a b}^{\max } / D_{n}<2 \text { Probable grain growth condition: NGG }
\end{aligned}
$$

The AGG conditions described above are somewhat arbitrary in nature and may vary for systems with very wide grain size distribution. In a static system with stationary mean grain $\left(D_{n}\right)$ size and pinning condition, it is sufficient to present $d / d t\left(D_{a b} / D_{n}\right)$ as a prediction of AGG. On the other hand, system with evolving mean grain size and pinning conditions, $D_{a b}^{\max } / D_{n}$ provides a much simpler representation. In order to present the evolution of AGG condition with time, $D_{a b}^{\max } / D_{n}$ is presented as the parameter presenting AGG probability in the studied system.

\subsection{Effects of precipitate size distribution on pin- ning}

In this section, a comparative study is presented in order to depict the effects of precipitate size distribution on Zener $\left(P_{Z}\right.$ or $\left.\bar{P}_{C}\right)$ and corner $\left(P_{C}\right.$ or $\left.\bar{P}_{C}\right)$ pinning. 

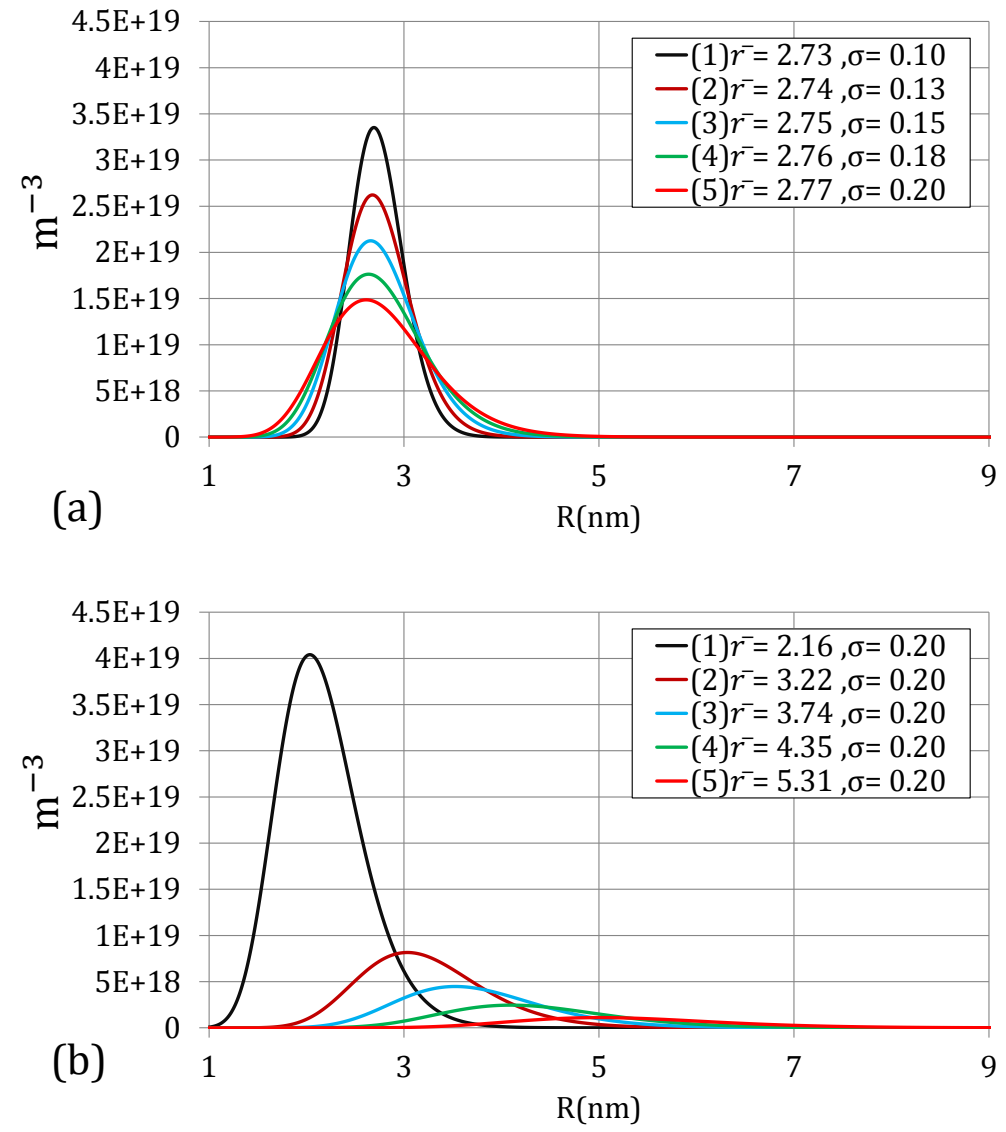

Figure 2.6: (a) Different shapes of precipitate size distribution varying in standard deviation $(\sigma)$ having the same mean radius $(\bar{r}=2.7 \mathrm{~nm})$ and total volume fraction $\left(f_{v}=0.0001\right)$. (b) Different shapes of precipitate size distribution with same standard deviation $(\sigma=0.2)$ and volume fraction $\left(f_{v}=0.0001\right)$ but varying in mean radius $(\bar{r})$. Here, $\mathrm{m}^{-3}$ refers to the number of precipitate per cubic meter and the mean grain diameter, $D_{n}=10 \mu \mathrm{m}$. 
Two hypothetical conditions of precipitate size distribution are assumed: (1) The precipitate volume fraction $\left(f_{v}=0.0001\right)$ and mean radius $(\bar{r}=2.7 \mathrm{~nm})$ are kept constant, but the distribution is increasingly wider varying in standard deviation $(\sigma)$ (see figure 2.6(a) from curve 1 to 5 ) and (2) the precipitate volume fraction $\left(f_{v}=0.0001\right)$ and standard deviation $(\sigma=0.2)$ are kept constant, but the mean radius is gradually increased (see figure 2.6(b) from curve 1 to 5).

The Zener pinning pressure $\left(\bar{P}_{Z}\right)$ considering the precipitate mean radius and volume fraction is calculated using expression 2.6 and $\left(P_{Z}\right)$ considering precipitate size distribution is calculated using the expression 2.7. The corner pinning $\left(\bar{P}_{C}\right)$ considering precipitate mean radius $(\bar{r})$ is calculated using the equations 2.15 or 2.16 and $\left(P_{C}\right)$ considering precipitate size distribution is calculated by expression 2.17 or 2.18. Here for every case, the mean grain diameter is taken as $D_{n}=10 \mu \mathrm{m}$ and the corner pinning amplification factor $\alpha=2.0$.

All the calculated pinning pressure (both Zener and corner) using the precipitate size distributions presented in figure 2.6 (a) are shown in figure 2.7 (a) and (b). Similarly, calculated pinning pressure using the precipitate size distributions presented in figure 2.6 (b) are given in figure 2.7 (c) and (d).

In figure 2.7 (a) and (b), effects of precipitate size distribution's standard deviation $(\sigma)$ on both Zener and corner pinning are presented. From figure 2.7 (a), it can be observed that Zener pinning pressure calculated utilizing the mean radius and size distribution show a gradual decrease with increasing standard deviation. In comparison with the mean radius consideration Zener pinning pressure calculated using whole size distribution show rather an accelerated decrease. The decreases in Zener pinning calculated using mean radius approach is due to the slight increases in mean radius (theoretically it should remain constant). Between standard deviation $(\sigma)$ of 0.1 and 0.2 the difference increases from $\sim 1000$ to $\sim 4000$ Pascal.

The reason for lower Zener pinning with size distribution consideration can be traced back to the increase in precipitate number density with a mono-disperse (mean radius) precipitate assumption. In a multi class precipitate size distribution, the total number of precipitate is distributed over a range of precipitate radius classes. A constant precipitate volume fraction impose restriction on the number of precipitates that can be allocated in a particular radius class. Due to the presence of larger precipitate radius class than the mean radius approach, a overall decrease in number density occurs. As a consequence, the decrease in precipitate number density reflects on the decrease in Zener pinning calculated using precipitate size distribution.

Corner pinning pressure taking into account the mean radius $\left(\bar{P}_{C}\right)$ (see figure 2.7 (b)) shows insensitivity (quite constant value) with the change in standard deviation $(\sigma)$ of size distribution. On the other hand, corner pinning pressure calculated 

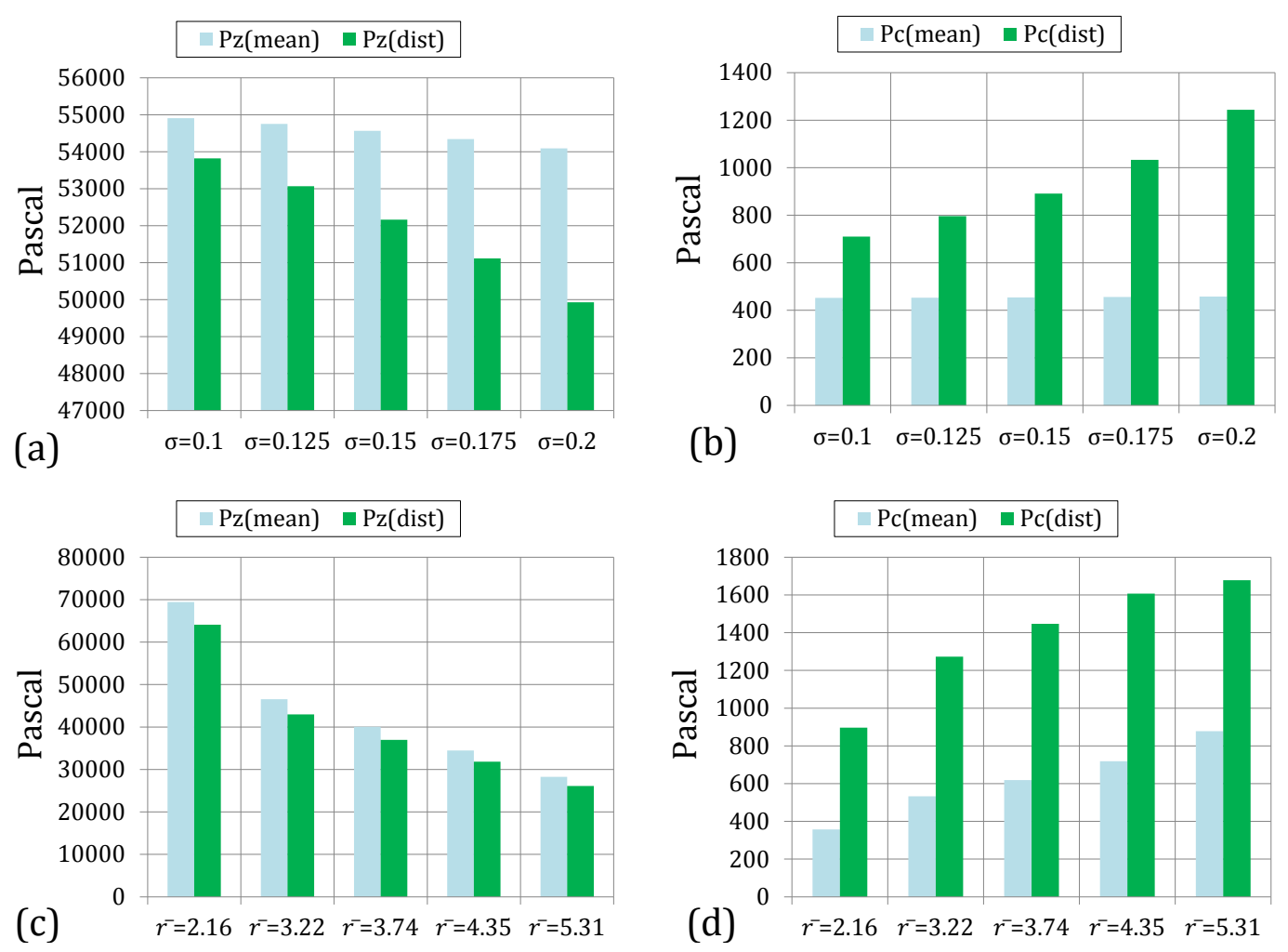

Figure 2.7: Comparison between Zener and corner pinning calculated using precipitate mean radius and size distribution for different characteristics of precipitate size distribution. Increasing standard deviation $(\sigma=0.1$ to 0.2$)$ of the distribution: (a) Zener pinning: $\bar{P}_{Z}$ and $P_{Z}$ considering precipitate mean radius and size distribution, (b) Corner pinning: $\bar{P}_{C}$ and $P_{C}$ considering precipitate mean radius and size distribution. Increasing mean radius $(\bar{r}=2.16 \mathrm{~nm}$ to $5.31 \mathrm{~nm})$ of the distribution: (c) Zener pinning: $\bar{P}_{Z}$ and $P_{Z}$ considering precipitate mean radius and size distribution, (d) Corner pinning: $\bar{P}_{C}$ and $P_{C}$ considering precipitate mean radius and size distribution. 
using precipitate size distribution $\left(P_{C}\right)$ shows an increase in value with increasing $\sigma$. As it is assumed that largest precipitate preferentially reside in the corner points, with an increase larger radii class number density (increase in $\sigma$ ), increases the overall corner pinning pressure (distribution consideration).

In figure $2.7(\mathrm{c})$ and $(\mathrm{d})$, effects of precipitate mean radius $(\bar{r})$ having same standard deviation is presented. Zener pinning $\left(\bar{P}_{Z}\right)$ considering precipitate mean radius and size distribution $\left(P_{Z}\right)$ (see figure $2.7(\mathrm{c})$ ) shows a decreasing trend with increasing mean radius $(\bar{r}) . \bar{P}_{Z}$ and $P_{Z}$ shows a small difference for all the studied conditions. This shows that for increasing precipitate mean radius, quite similar quantity of Zener pinning is obtained from both mean radius and size distribution considerations. As the mean radius increases, in order to have same volume fraction, the overall number density becomes smaller. The decrease in Zener pinning (both mean and size distribution consideration) is due to the decrease in precipitate number density in both overall size distribution and mean radius approach.

Increasing precipitate mean radius, increases both the corner pinning pressures for precipitate size distribution $\left(P_{C}\right)$ and mean radius consideration $\left(\bar{P}_{C}\right)$ (see figure $2.7(\mathrm{~d})$ ). In size distribution consideration, comparatively (in comparison with mean radius approach) larger precipitate participate in the corner pinning. As the number of corner points remain unchanged, increase in corner pinning pressure in size distribution consideration is solely due to the increases in precipitate radius.

From the above discussion, it can be said that the precipitate mean radius consideration makes both Zener $\left(\bar{P}_{Z}\right)$ and corner pinning $\left(\bar{P}_{C}\right)$ insensitive to the distribution width (increasing or decreasing standard deviation). The pinning pressure is then only dependent on precipitate number density (dependent on volume fraction) in the system. Zener pinning $\left(P_{Z}\right)$ decreases for precipitate size distribution consideration and also with an increasing distribution width $(\sigma)$. On the other hand, corner pinning pressure $\left(P_{C}\right)$ shows an increase in value. Increase in precipitate mean radius (having same standard deviation) shows a similar trend in Zener pinning calculated using mean radius and size distribution approach. It can be seen that the difference between Zener pinning for both approach rather decreases with increasing mean radius. Quite opposite trend can be seen for the corner pinning pressures $\left(\bar{P}_{C}\right.$ and $\left.P_{C}\right) . \bar{P}_{C}$ and $P_{C}$ both increases with increasing mean radius of the size distribution. It is also noticeable that the difference between corner pinnings $\left(\bar{P}_{C}\right.$ and $\left.P_{C}\right)$ also increases with increasing mean radius. It can be concluded from the observation of figure 2.7 that precipitate size distribution consideration decreases the calculated Zener pinning in comparison with the mean radius approach. In the mean time, corner pinning calculated using distribution rather shows an increase for all the presented cases. 

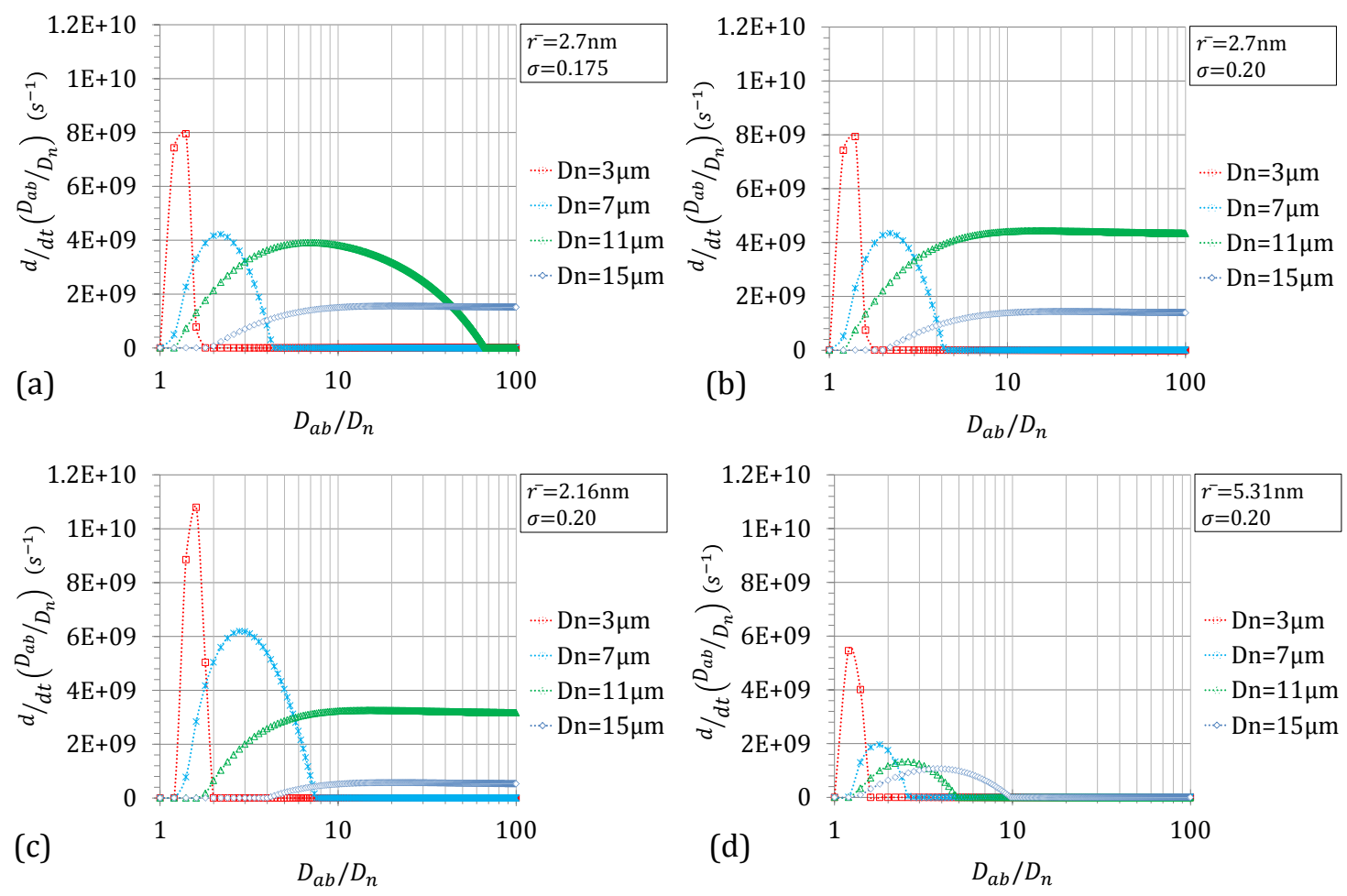

Figure 2.8: Effects of precipitate size distribution's standard deviation $(\sigma)$ and mean grain size $\left(D_{n}\right)$ on abnormal grain growth: (a) standard deviation, $\sigma=0.175$ and (b) standard deviation $\sigma=0.2$. Effects of precipitate mean radius $(\bar{r})$ and mean grain size $\left(D_{n}\right)$ on abnormal grain growth: (c) mean radius, $\bar{r}=2.16 \mathrm{~nm}$ and (d)mean radius, $\bar{r}=5.31 \mathrm{~nm}$. Here, the precipitate volume fraction $f_{v}=0.0001$, the AGG model parameter $\mu=0.56, \lambda=0.6$ and the grain boundary mobility assumed to be $M=1$.

\subsection{Effects of precipitate size distribution on AGG}

In the following discussion, emphasis is given to understand the effects of different precipitate size distribution (constant volume fraction) in terms of standard deviation $(\sigma)$ and arithmetic mean $(\bar{r})$ on AGG condition. A system with preexisting AGG condition is chosen. This gives us opportunity to realize how the precipitation size distribution characteristics impact on the grain growth condition. The mean grain size is also varied to study the effects of initial austenite grain size on AGG.

It can be observed from figure 2.8 (a) and (b), that with an increase in mean grain size there is an increase in maximum grain size that is susceptible to abnormal growth. There is no possibility of AGG in the microstructure where $D_{n}=1 \mu \mathrm{m}$ because $d / d t\left(D_{a b} / D_{n}\right)$ is positive within $D_{a b}^{\max } / D_{n}=1$ to $<2$ for both $\sigma=0.175$ and 0.2. As the grain sizes close to the mean grain size have the highest relative growth rate, microstructure has lowest probability to experience AGG. Although the grain sizes larger than $1 \mu \mathrm{m}$ showing an increase in $D_{a b}^{\max }$, a decrease in relative 
growth rate can be observed. Figure 2.8 (a) and (b) suggest, $D_{n}=3,7$ and $15 \mu \mathrm{m}$ showing quite similar larger grains relative growth rate $\left(d / d t\left(D_{a b} / D_{n}\right)\right)$ but for $D_{n}=11 \mu \mathrm{m}$ we can observe an increase in $D_{a b}^{\max }$. Finally, it can be stated that an increase in the precipitate size distribution's standard deviation $(\sigma)$, enlarge $D_{a b}^{\min }$ to $D_{a b}^{\max }$ range for a particular mean grain size.

An increase in precipitate size distribution's standard deviation effectively increases the distribution width. It is conceivable that wider size distribution will have higher number of precipitates in the largest radii classes. Number of precipitates increasing in the larger radii classes contribute in increasing the corner point pinning. Besides that with increasing distribution width, comparatively larger precipitates also participate in the Zener pinning and consequently enhancing it (see figure 2.7 (a) and (b)). A total increase in pinning pressure $\left(P_{Z}+P_{C}\right)$, leads to a decrease in the normal grain growth rate $\left(d D_{n} / d t\right)$. As a result, the $D_{a b}^{\min }$ to $D_{a b}^{\max }$ range of AGG susceptible grain size enlarges.

In figure 2.8 (c), (b) and (d), the effects of precipitate mean radius (associated with increase in number of larger precipitates) for four different mean grain sizes ( $D_{n}=3,7,11$ and $15 \mu \mathrm{m}$ ) are presented. The change in AGG condition with an increasing $D_{n}$ is similar to the one observed for standard deviation. It can be seen that an increase in the precipitate mean radius $(\bar{r})$, decreases the relative growth rate $\left(d / d t\left(D_{a b} / D_{n}\right)\right)$ of the larger grains. As the precipitate volume fraction $\left(f_{v}=\right.$ $0.001)$ is kept constant for both cases, the reason for increase in the $d / d t\left(D_{a b} / D_{n}\right)$ can be associated with the decrease in total pinning pressure in the system (see figure $2.7(\mathrm{c})$ and $(\mathrm{d})$ ). Besides the decrease in larger grain's relative growth rate, a reduction in $D_{a b}^{\min }$ to $D_{a b}^{\max }$ range can also be seen. From this comparative study, it can be said that in a pre-existing AGG condition, increase in the precipitate mean radius $(\bar{r})$, reduces the relative growth rate and also $D_{a b}^{\max }$ of grains having size advantage over the mean. Thus, a reduction in AGG probability can be expected.

\subsection{Effects of mean grain size on abnormal grain growth condition}

Effects of mean austenite grain size $\left(D_{n}\right)$ on AGG conditions is presented for a constant precipitation condition in figure 2.9. In this study, the precipitate volume fraction and size distribution is chosen in such a way that an AGG condition always exits. In the 3-D plot, $d / d t\left(D_{a b} / D_{n}\right)$ is plotted on the z-axis, mean grain diameter $D_{n}$ on $\mathrm{x}$-axis and $D_{a b} / D_{n}$ on y-axis. Here, $D_{a b}$ refers to the pre-existing larger grains in the microstructure.

It can be seen from figure 2.9, that is a pre-existing AGG favorable precipitation 


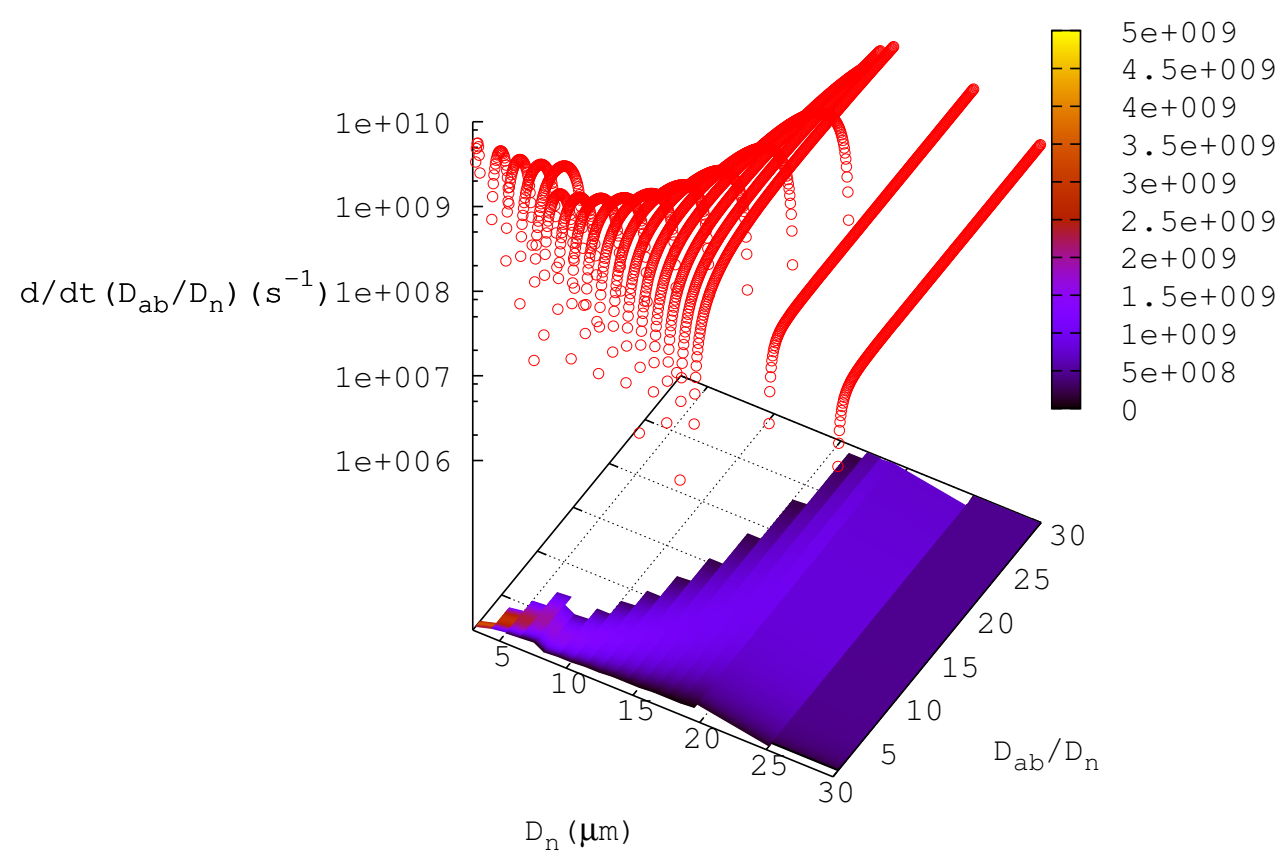

Figure 2.9: Effects of mean grain size $\left(D_{n}\right)$ on abnormal grain growth condition $\left(d / d t\left(D_{a b} / D_{n}\right)\right)$. Here, the precipitate volume fraction $f_{v}=0.00033$ and the mean radius $\bar{r}=26 \mathrm{~nm}$. AGG model parameter $\mu=0.56$ and $\lambda=0.6 . d / d t\left(D_{a b} / D_{n}\right)$ value is shown on the $\mathrm{x}-\mathrm{y}$ plane marked by the right hand side scale.

state, an increase in the mean grain diameter increases the maximum range of grain sizes $\left(D_{a b}^{\min }\right.$ to $\left.D_{a b}^{\max }\right)$. In every case, $D_{a b}^{\min }$ retain a value close to $D_{n}$ and $D_{a b}^{\max }$ increases with increasing mean grain size $\left(D_{n}\right) . D_{a b}^{\max }$ remains below 2 (no AGG) for mean grain sizes $1-3 \mu \mathrm{m}$. A gradual increase in the $D_{a b}^{\max }$ can be observed up to $D_{n}=19 \mu \mathrm{m}$ and above this $D_{a b}^{\max }$ goes to infinity. Equation 2.21 suggests that an increase in the mean grain size decreases the mean grain growth pressure. A decrease in mean grain growth rate $\left(d D_{n} / d t\right)$ increases the relative growth rate $\left(d / d t\left(D_{a b} / D n\right)\right)$. As a result $D_{a b}^{\max }$ get elevated. In fact, the mean grain growth rate has to reach zero $\left(P_{D_{n}}<P_{Z}+P_{C}\right)$ for the relative growth rate to reach a relatively constant value $\left(D_{a b}^{\max } \rightarrow \infty\right)$. In this particular state of a microstructure, the smaller grains (diameter close to the mean) remain immobile and the larger grains in the system are free to grow and consume its neighbors.

It can be concluded that an increase in mean grain size increase the maximum grain size $\left(D_{a b}^{\max }\right)$ with probability to experience AGG. When grains in a system with constant precipitation state experience significant coarsening (increase in mean diameter), probability of AGG occurrence increases. System having particular density and characteristics of precipitate size distribution, have a critical mean grain diameter for which probability of AGG occurrence is maximum. 
Table 2.1: FeVNbCN alloy composition is atomic \%.

\begin{tabular}{ccccccc}
\hline Element & $\mathrm{C}$ & $\mathrm{Si}$ & $\mathrm{Mn}$ & $\mathrm{S}$ & $\mathrm{Ni}$ & $\mathrm{Cr}$ \\
at\% & 2.2 & 0.055 & 0.088 & 0.029 & 0.021 & 0.052 \\
\hline & & & & & & \\
\hline Element & $\mathrm{Mo}$ & $\mathrm{Ti}$ & $\mathrm{Nb}$ & $\mathrm{V}$ & $\mathrm{N}$ & \\
at\% & 0.004 & 0.0011 & 0.0071 & 0.217 & 0.025 & \\
\hline
\end{tabular}

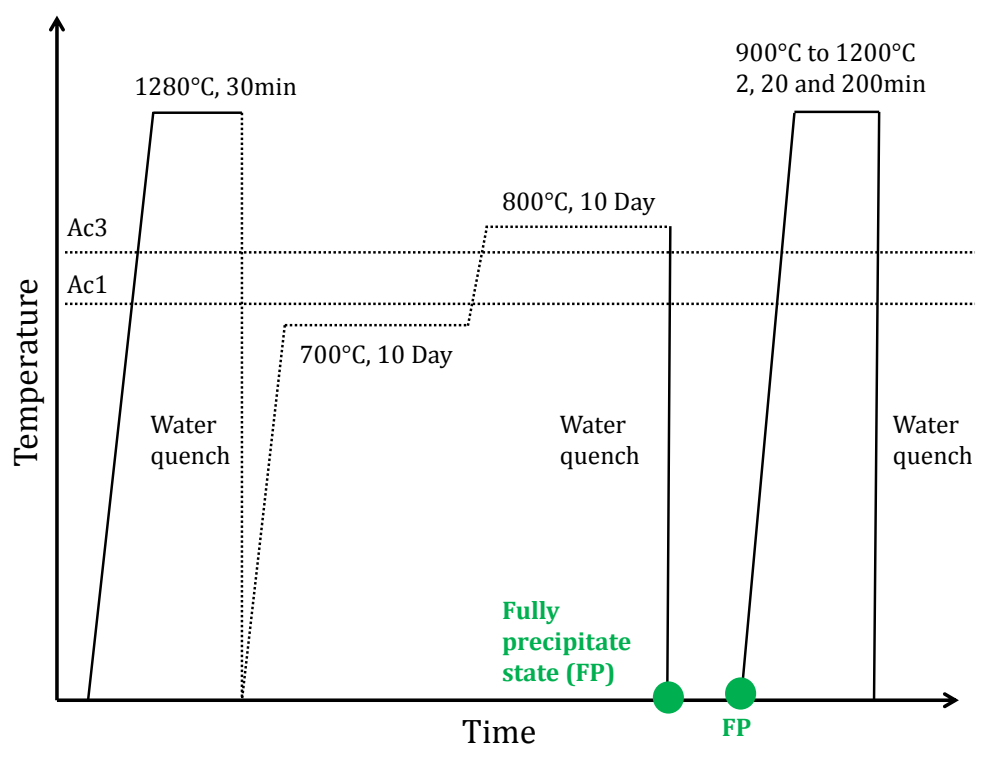

Figure 2.10: Heat treatment cycle performed on the FeVNbCN model alloy.

\subsection{Application}

In this section, validity of the presented abnormal grain growth model is tested by comparing model predictions with experimental observations. A model alloy is chosen and the AGG model is applied in order to predict grain growth condition (Normal/Abnormal) in the alloy. The following discussion consists of alloy description and model application methodology in details.

\subsubsection{Materials and heat treatment}

In table 2.1, FeVNbCN alloy composition in atomic $\%$ is given. The model alloy is first subjected to a solutionizing treatment at $1280^{\circ} \mathrm{C}$ for 30 min followed by a rapid quench in water. After the solutionizing treatment, samples of $8 \mathrm{~mm}$ in size are heat treated below the $A C_{1}$ at $700^{\circ} \mathrm{C}$ for 10 days, followed by 10 days of heat treatment at $800^{\circ} \mathrm{C}$ above $A C_{3}$ and finally water quenched. The $\mathrm{VCN}$ and $\mathrm{NbCN}$ precipitate nucleation and growth occurred in the ferrite $(\alpha)$ phase during the heat treatment below $A C_{1}$. The heat treatment above $A C_{3}$ allowed coarsening of the precipitates in the austenite $(\gamma)$ phase. In FeVNbCN model alloy a long heat 
Table 2.2: A list of all the model parameters used in calculation.

\begin{tabular}{cccccccc}
\hline$k_{s}[9]$ & $k_{v}[9]$ & $\lambda_{n}$ & $\gamma_{g}$ & $\alpha$ & $K_{V}[9]$ & $K_{A}[9]$ & $\mu$ \\
$\pi$ & $4 \pi / 3$ & 0.6 & 1 & 2 & $(1.1)^{3} \pi / 6$ & $(1.1)^{2} \pi / 2$ & 0.54 \\
\hline
\end{tabular}

treatment time helped to achieve a "Fully Precipitated (FP)" state with a relatively large precipitates $(>100 \mathrm{~nm})$. The $\mathrm{FP}$ state is then heat treated above the $A C_{3}$ at different temperatures and times, in order to study the effects of dissolving precipitates on the grain growth behavior.

\subsubsection{Results and discussion}

\subsubsection{Experimental observation}

In figure 2.11 and 2.12 (a), (c) and (e), the VCN and NbCN precipitates distribution densities normalized with volume fraction are presented. Here, the number of precipitates in a class $i\left(m^{-3}\right)$ is divided by the class width $\left(\Delta r_{i}\right)$. The corresponding grain size distribution is presented in the figure 2.11 and 2.12 (b), (d) and (f). The grain size distribution is presented as volume fraction $\left(v_{f_{i}}=N_{i} D_{i}^{3} / \sum_{i}^{n} N_{i} D_{i}^{3}\right)$ of individual class in a grain size distribution.

Heat treatment done at $900^{\circ} \mathrm{C}$ for 2,20 and 200 min showed normal $(\gamma)$ grain growth. It can be confirmed from the grain size distribution, as in figure 2.11 (a), (c) and (e) and also from micrograph presented in figure $2.13 A_{1}, A_{2}$ and $A_{3}$ that the largest diameter classes are occupying less or equal volume fraction than the smaller classes. On the other hand, heat treatment done at $1050^{\circ} \mathrm{C}$ for 2,20 and 200 min showed abnormal grain $(\gamma)$ growth. It can be seen from the grain size distribution in figure 2.12 (a), (c) and (e) and also from micrograph presented in figure $2.13 B_{1}, B_{2}$ and $B_{3}$ that the largest diameter classes are occupying higher volume fraction in comparison with the smaller ones (presence of AGG).

\subsubsection{Calculation procedure}

The AGG model is applied to all the states (described in the previous section) of FeVNbCN model alloy, having different types of grain growth conditions. Precipitates distribution and mean grain size of the specimens heat treated at $900^{\circ} \mathrm{C}$ and $1050^{\circ} \mathrm{C}$ for 2,20 and $200 \mathrm{~min}$ are taken as input parameters. The model and experimental results are compared to verify the model predictions and estimate its effectiveness in predicting AGG or NGG occurrence.

In the studied FeVNbCN model alloy, Zener pinning and corner pinning are taken into account to estimate the total pinning pressure in the system. Pinning pressures are calculated using expressions 2.18. The pinning induced grain growth 

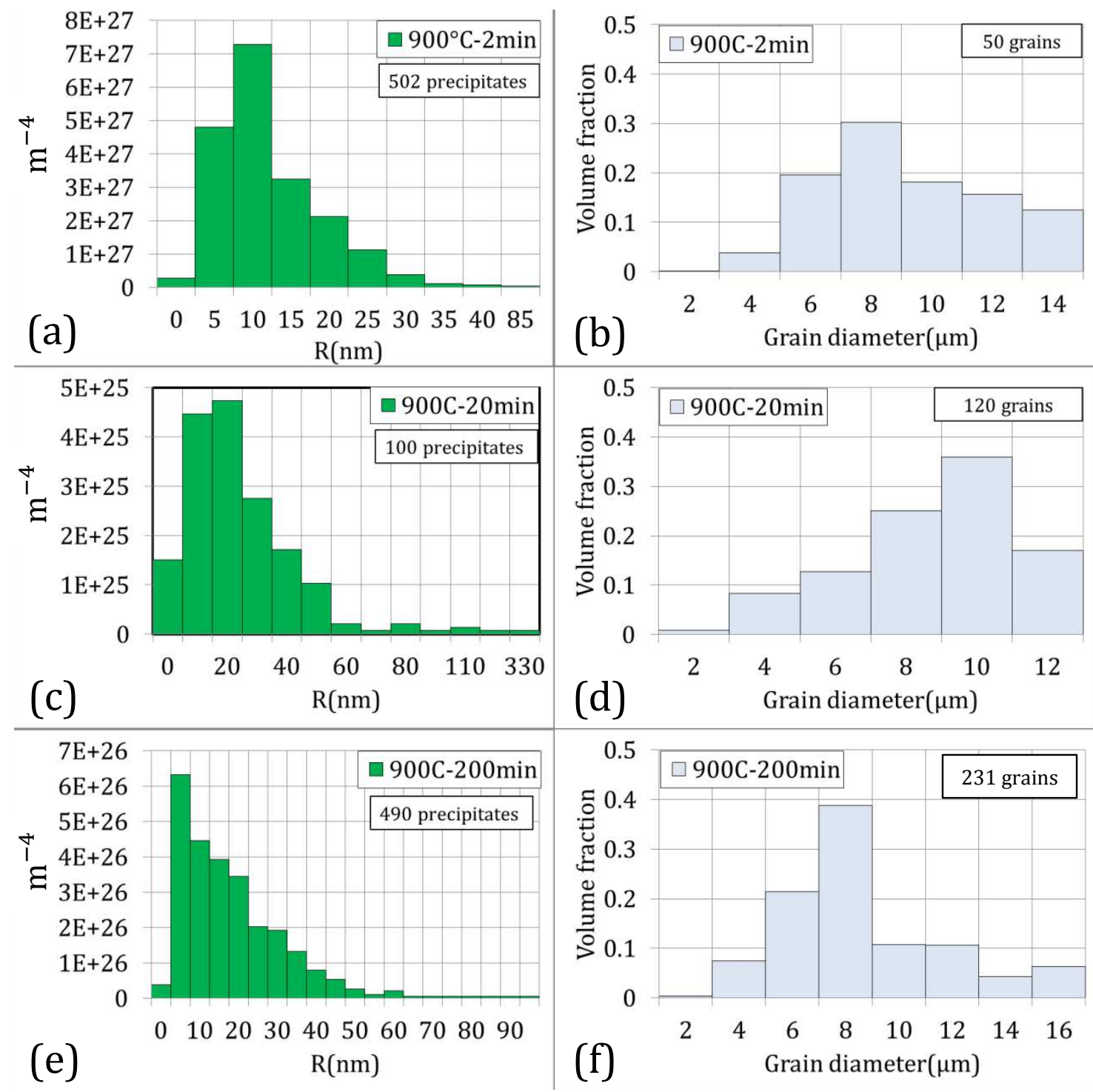

Figure 2.11: Precipitate and grain size distribution of the FP state heat treated at $900^{\circ} \mathrm{C}$ for different holding times. Precipitate size distribution of the FP state heat treated at $900^{\circ} \mathrm{C}$ for (a) $2 \mathrm{~min}$, (c) $20 \mathrm{~min}$ and (e) $200 \mathrm{~min}$. Grain size distribution of the FP state heat treated at $900^{\circ} \mathrm{C}$ for (b) $2 \mathrm{~min}$, (d) $20 \mathrm{~min}$ and (f) $200 \mathrm{~min}$. 

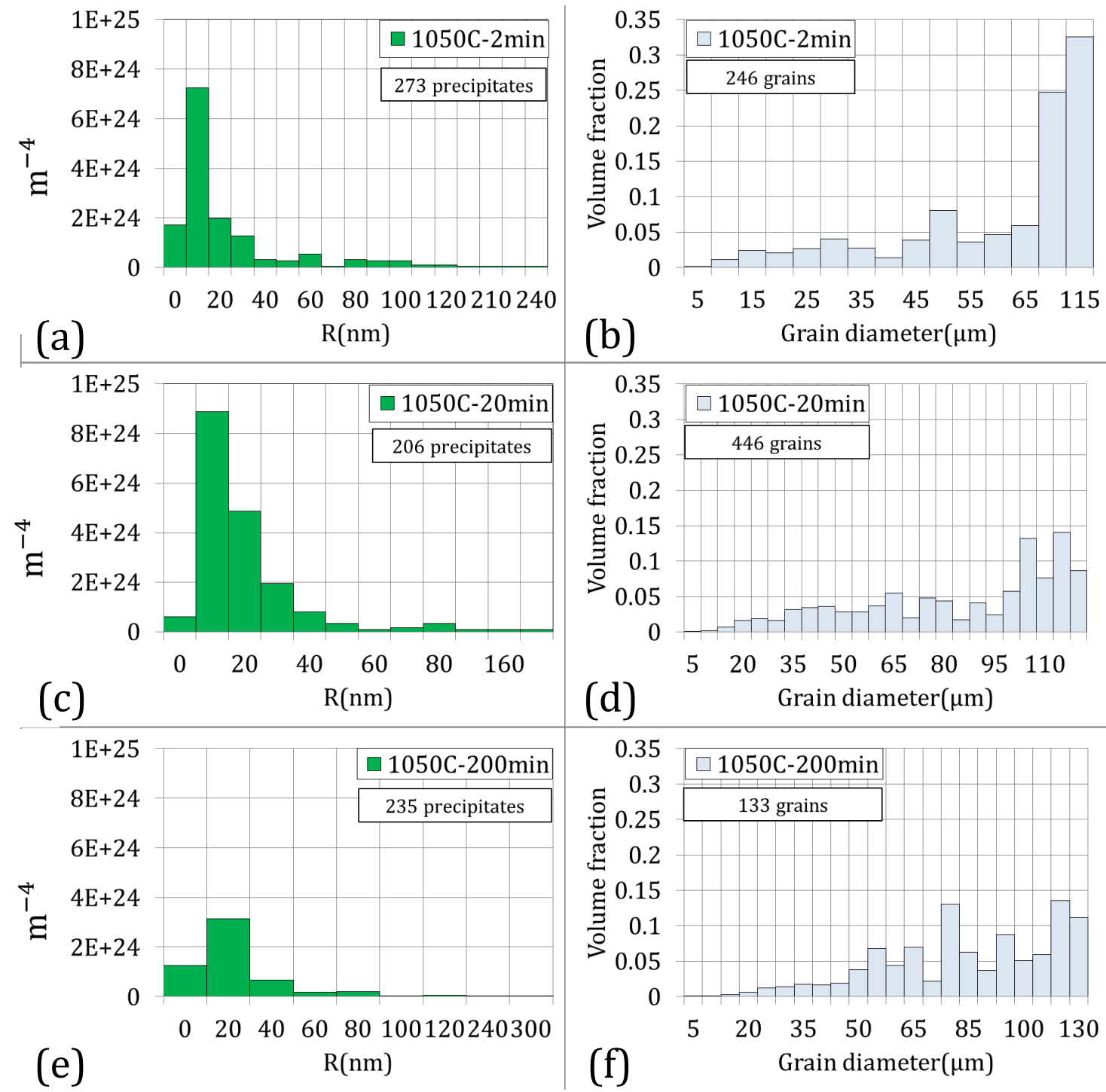

Figure 2.12: Precipitate and grain size distribution of the FP state heat treated at $1050^{\circ} \mathrm{C}$ for different holding times. Precipitate size distribution of the FP state heat treated at $1050^{\circ} \mathrm{C}$ for (a) $2 \mathrm{~min}$, (c) $20 \mathrm{~min}$ and (e) $200 \mathrm{~min}$. Grain size distribution of the FP state heat treated at $1050^{\circ} \mathrm{C}$ for (b) 2 min ,(d) 20 min and (f) $200 \mathrm{~min}$. 

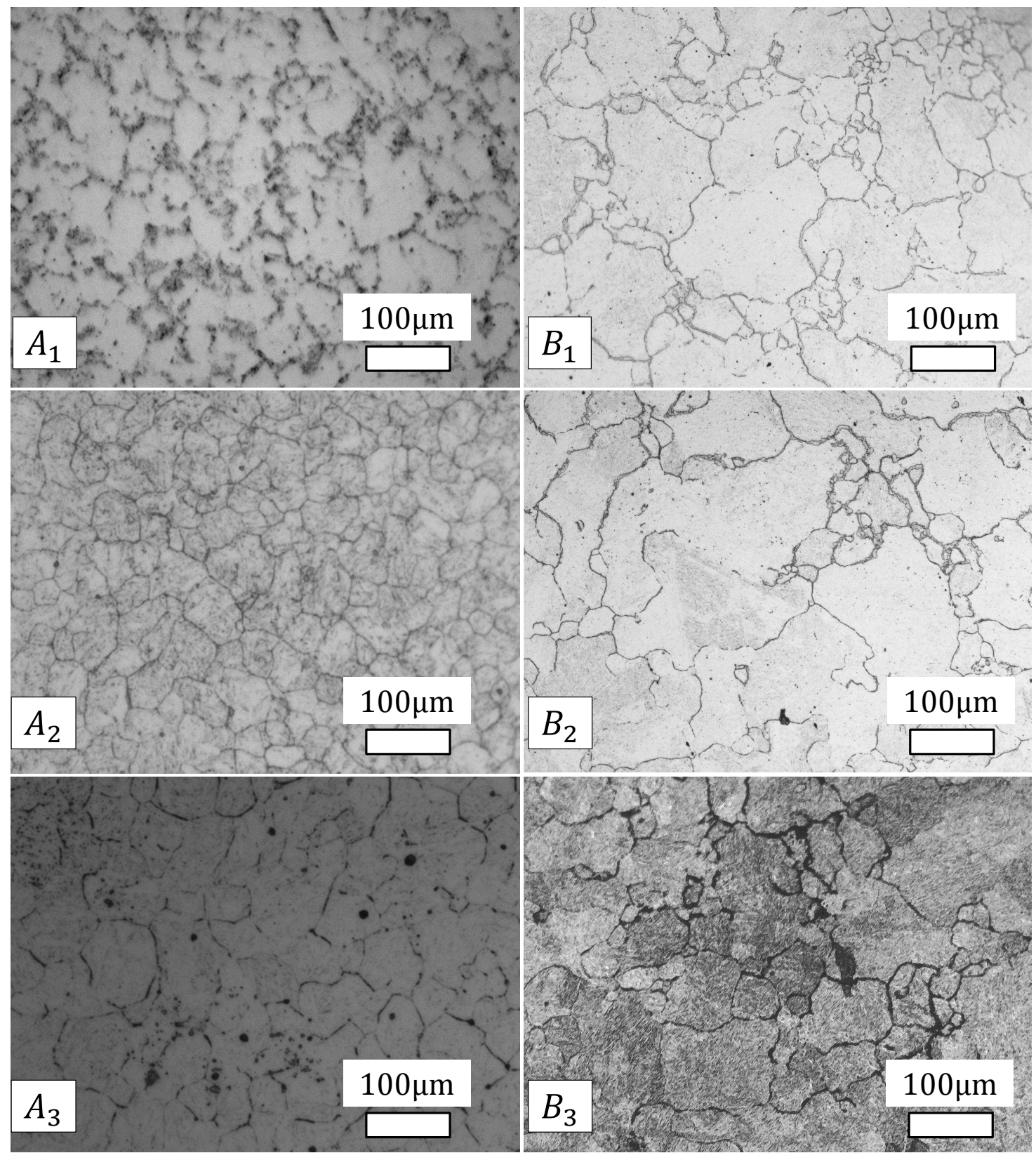

Figure 2.13: Micrograph of austenite grains in FeVNbCN model alloy heat treated at $900^{\circ} \mathrm{C}$ for $\left(A_{1}\right): 2 \mathrm{~min},\left(A_{2}\right): 20 \mathrm{~min}$ and $\left(A_{3}\right): 200 \mathrm{~min}$ and heat treated at $1050^{\circ} \mathrm{C}$ for $\left(B_{1}\right): 2 \mathrm{~min},\left(B_{2}\right): 20 \mathrm{~min}$ and $\left(B_{3}\right): 200 \mathrm{~min}$. 


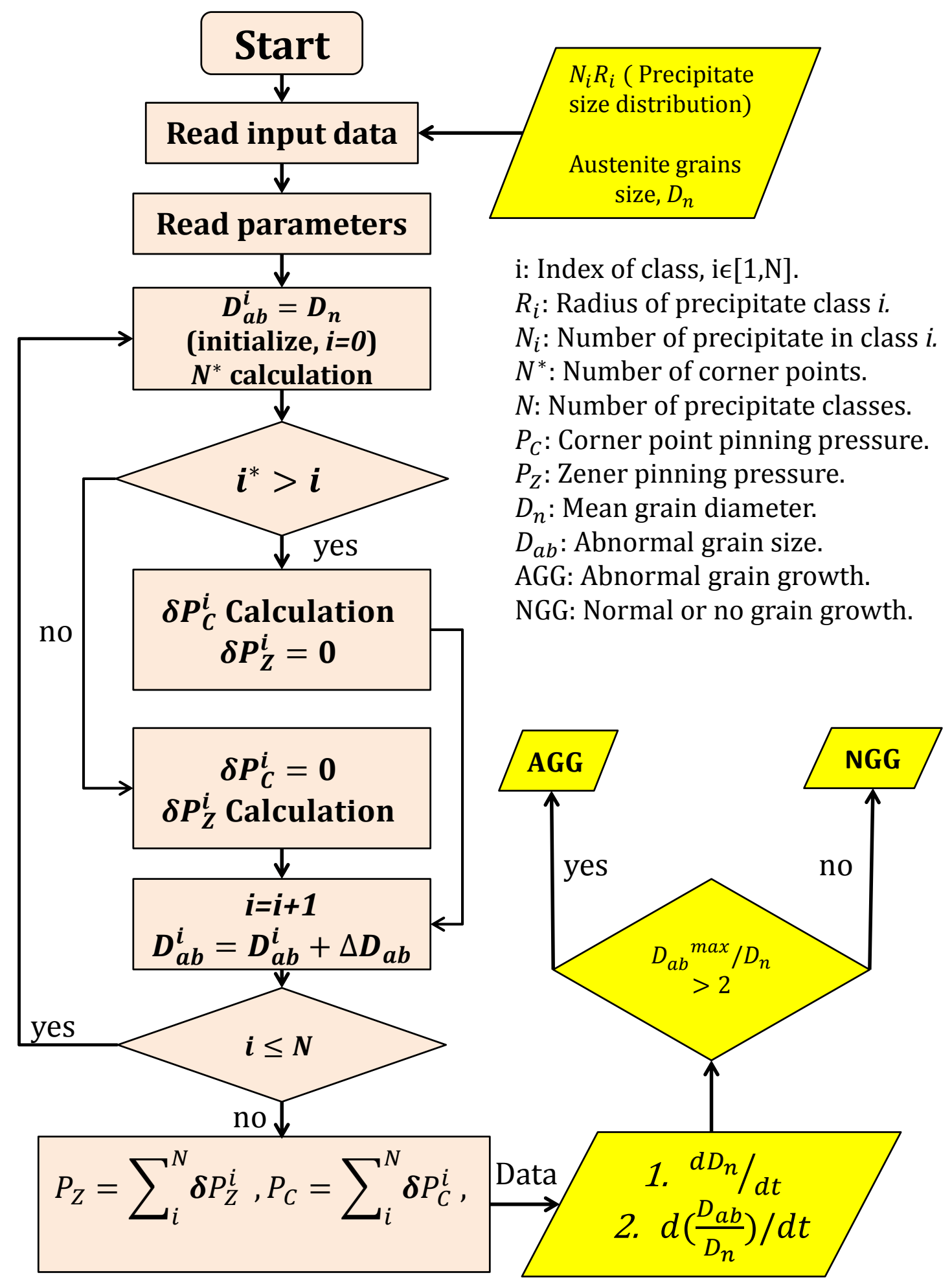

Figure 2.14: A schematic representation of the AGG model calculation procedure. 
Table 2.3: A Comparison between the AGG model predictions and the experimental observations.

\begin{tabular}{|c|c|c|c|}
\hline Heat treatment & $D_{a b}^{\max } / D_{n}$ & Model prediction & Experimental observation \\
\hline $900^{\circ} \mathrm{C} 2 \mathrm{~min}$ & 0 & NGG & NGG \\
\hline $900^{\circ} \mathrm{C} 20 \mathrm{~min}$ & 0 & NGG & NGG \\
\hline $900^{\circ} \mathrm{C} 200 \mathrm{~min}$ & 0 & NGG & NGG \\
\hline $1050^{\circ} \mathrm{C} 2 \mathrm{~min}$ & 2.245 & AGG & AGG \\
\hline $1050^{\circ} \mathrm{C} 20 \mathrm{~min}$ & $\infty$ & AGG & AGG \\
\hline $1050^{\circ} \mathrm{C} 200 \mathrm{~min}$ & $\infty$ & $\mathrm{AGG}$ & \\
\hline
\end{tabular}

instability in the system is then calculated using expressions 2.20 and 2.21. A step by step procedure (figure 2.14) can be described as follows. The model parameters used are listed in table 2.2:

1. The number of corner points in the system is calculated using, $N^{*}=K_{0} /\left(K_{V} D_{n}^{3}\right)$ relation where $D_{n}$ is the mean grain diameter.

2. Starting from the precipitate class with largest radius (furthest right of the distribution), the number of corner points available is filled with corresponding and also subsequent classes of precipitates. The corner point pinning pressure for the individual class is calculated using expression 2.17.

3. After filling all the corner points with the available largest radii precipitate classes, the rest of the classes (if available after filling the corner points) of precipitate are taken into account for Zener pinning. Zener pinning pressure for individual class is calculated using expression 2.18.

4. Growth pressure of the individual $D_{a b}^{i}$ starting from $D_{a b}^{1}=D_{n}$ to $D_{a b}^{e n d}=$ $100 \times D_{n}$ is calculated following the expression 2.20. In parallel, growth pressure of the mean size grain is calculated using the expression 2.21. Growth rate of the large grains $d D_{a b} / d t$ and the normal grains $d D_{n} / d t$ are used in the expression 2.22 to measure instability of grain growth condition (AGG or NGG) in the system. Finally, the minimum $D_{a b}^{\min }$ and the maximum $D_{a b}^{\max }$ grains are identified, within which the second condition of AGG $\left(d / d t\left(D_{a b} / D_{n}\right)>\right.$ $0)$ is true. If $D_{a b}^{\max } / D_{n}>2$, the microstructure is identified with probability of experiencing AGG.

\subsubsection{Model prediction}

The AGG model results are presented in figure 2.15. Model predictions of the heat treatment done at $900^{\circ} \mathrm{C}$ for 2,20 and $200 \mathrm{~min}$ are shown in the figure 2.15 (a) to (c). In these states both the AGG conditions $d D_{a b} / d t=0$ and $d\left(D_{a b} / D_{n}\right) / d t=0$ are not met and as a results $D_{a b}^{\max } / D_{n}=0$, thus no probability of AGG. These 



Figure 2.15: AGG model prediction for different heat treated states of FeVNbCN model alloy. AGG model results for the heat treatment done at $900^{\circ} \mathrm{C}$ for (a) 2 $\min \left(\right.$ b) $20 \mathrm{~min}$ (c) $200 \mathrm{~min}$ and at $1050^{\circ} \mathrm{C}$ for (d) $2 \mathrm{~min}$ (e) $20 \mathrm{~min}$ and (f) 200 min. Here, the model parameters $\lambda=0.6$ and $\mu=0.54$. 
results are in agreement with the experimental observations presented in the figure 2.11. Model predictions of the heat treatment done at $1050^{\circ} \mathrm{C}$ for 2,20 and 200 min are presented in figure 2.15 (d) to (f). Here, the model results suggest that both the AGG conditions $d D_{a b} / d t>0$ and $d\left(D_{a b} / D_{n}\right) / d t>0$ are fulfilled and for all the heat treatments at $1050^{\circ} \mathrm{C}, D_{a b}^{\max } / D_{n}>2$. So, the model predicts that there is a probability of $\mathrm{AGG}$ occurrence in heat treatment done at $1050^{\circ} \mathrm{C}$ for different times. Again in comparison with the experimental results presented in figure 2.12, model predictions are fully in agreement. A summary of model predictions and experimental observations of all the heat treated states are presented in table 2.3.

\subsection{Conclusions from this chapter}

In the present model, AGG is handled with simple geometrical assumptions: one abnormal grain is surrounded by normal grains. If only Zener pinning occurs, a mechanism map for normal and abnormal grain growth is presented. It shows that AGG occurs in a moderate pinning condition. The proposed model is improved in order to take into account corner pinning (pinning at the quadruple or the triple point). The different parameters influencing model characteristics are discussed in details. A clear picture of Zener and corner pinning sensitivity to precipitate size distribution is also depicted. It is found that, the precipitate size distribution consideration decreases the calculated Zener pinning in comparison with the mean radius consideration. Besides that, the corner pinning contribution to the total pinning force is found to be much lower than Zener pinning. Finally, the model is applied to a low alloy steel (FeVNbCN), heat treated at different temperatures and having varying precipitate size distributions. Taking into account only one set of parameters, the model is able to predict AGG and NGG for all the studied heat treatments. This is an indication of the versatility of the AGG model in predicting grain growth condition in low alloy steel. 
Cette thèse est accessible à l'adresse : http://theses.insa-lyon.fr/publication/2013ISAL0094/these.pdf () [M.A. Razzak], [2013], INSA de Lyon, tous droits réservés 


\section{Chapter 3}

\section{Precipitation modeling}

In this chapter, a brief description of a precipitation model is presented. The objective of the model is to predict the precipitation state evolution with time and temperature in low alloy steels. In the present approach, precipitation state evolution with time and temperature is predicted in terms of volume fraction, precipitate size and distribution density.

There are several models available in the literatures. In this study, we are presenting a precipitation model based on Wagner and Kampmann [68] proposition. This model is based on Classical Nucleation and Growth Theories (CNGTs), capable of predicting precipitation kinetics in isothermal and also non-isothermal condition. CNGTs provide a complete framework where precipitate nucleation and growth rate are predicted provided with accurate thermodynamic description. In the present study, accurate thermodynamic description of the precipitates is determined experimentally.

Both homogeneous (in matrix) and heterogeneous precipitate growth (in grain boundary and triple point) are explored in the present study. A comparative picture of homogeneous and heterogenous precipitate growth kinetics is presented. Precipitates lying at the grain boundary and triple points are treated separately than the one lying in the matrix. The mean austenite grain diameter determines the number of precipitates that can lie at the grain boundaries. So, mean austenite grain diameter effect on heterogeneous precipitate growth kinetics is also presented.

Finally, the precipitation model is applied to two different industrial alloys. The model predictions are compared with experimental measurements. Agreements and disagreements with experimental observations are discussed to evaluate the model's effectiveness in predicting precipitation state in different isothermal heat treatments. 


\subsection{Model hypothesis}

The CNGTs based model presented in this work, is based on the following assumptions.

1. Precipitates are assumed to be spherical in shape. This ensures simplicity by presenting only one dimensional parameter (radius) to be handled in the calculation.

2. Interface equilibrium solute concentration is governed by the local equilibrium. The solute concentration is defined by phase equilibrium at the interface which is corrected by the Gibbs-Thompson effect.

3. Solute concentration is uniform far away from the precipitate-matrix interface.

4. Precipitate-matrix interface interaction with the solute atoms does not effect growth or dissolution. Precipitate growth is solely controlled by solute diffusion in the matrix.

5. Precipitate interfacial energy is assumed to be constant for precipitate of all radiuses.

6. Solute concentration profile around a precipitate is assumed to be stationary.

In the subsequent sections precipitation model is described in more details.

\subsection{Thermodynamic equilibrium}

In this section, thermodynamic equilibrium of precipitation is austenitic $(\gamma)$ state is discussed. All the expressions regarding thermodynamic driving force of a binary precipitate are detailed.

In order to generalized the expressions, a stoichiometric precipitate $A_{x} B_{y}$ can be assumed and it ( $A$ is substitutional and $B$ is interstitial atomic species) is expressed as $\theta$ phase. If $(x+y) n$ number of atoms are transferred from the solid solution to the $A_{x} B_{y}$ precipitate then the change in chemical energy can be expressed in terms of Gibbs energy at a constant Pressure $(P)$ and Temperature $(T)$ as follows:

$$
\begin{array}{rl}
d G_{n}^{\gamma}=x & d n \frac{\partial G_{n}^{\gamma}}{\partial n_{A}}+y d n \frac{\partial G_{n}^{\gamma}}{\partial n_{B}} \\
= & d n\left(x \mu_{A}^{\gamma}+y \mu_{B}^{\gamma}\right)
\end{array}
$$

Here, $\mu_{A}=\frac{\partial G_{n}^{\gamma}}{\partial n_{A}}$ and $\mu_{B}=\frac{\partial G_{n}^{\gamma}}{\partial n_{B}}$ are defined as the chemical potentials of element $A$ and $B$ in the solid solution. 
Assuming a regular solution, Gibbs energy of the solid solution can be expressed as follows:

$$
G_{0}^{\gamma}=n_{A}\left[G_{A}^{0}+k_{b} T \ln X_{A}\right]+n_{B}\left[G_{B}^{0}+k_{b} T \ln X_{B}\right]+\Omega \frac{n_{A} n_{B}}{n_{A}+n_{B}}
$$

Here, $X_{A}$ and $X_{B}$ are the mole fraction of element $A$ and $B$ and $\Omega$ is the atomic interaction parameter between element $A$ and $B$ in solid solution. Gibbs energy expression for the precipitating phase $\theta$ can be written as follows:

$$
d G_{n}^{\theta}=d n\left(x G_{A}^{0}+y G_{B}^{0}+x y \Omega_{B}^{A}+k_{b} T\left(x \ln X_{A}+y \ln X_{B}\right)\right)
$$

Here, $\Omega_{B}^{A}$ is the atomic interaction parameter between element $A$ and $B$ in the precipitate ( $\theta$ phase). In a thermodynamic equilibrium, global energy in the system remains unchanged.

$$
d G_{n}^{\gamma}+d G_{n}^{\theta}=0
$$

Using equation 3.1 to 3.5 , following expression can be written for driving force of the $A_{x} B_{y}$ precipitate.

$$
\Delta G^{\theta}=k_{b} T \ln \left(\left[X_{A}\right]^{x}\left[X_{B}\right]^{y}\right)
$$

The right hand side of equation 3.6 is known as the solubility product of $A_{x} B_{y}$ precipitate in a dilute solid solution. Considering the assumption that equilibrium exits between the precipitate and matrix interface (equilibrium compositions at the interface is $X_{A}^{I}$ and $X_{B}^{I}$ ), solubility product can be expressed in terms two parameters $A^{a t \%}$ and $B^{a t \%}$ as follows:

$$
\ln \left(K_{s}\right)=\ln \left(\left[X_{A}\right]^{x}\left[X_{B}\right]^{y}\right)=-\frac{A^{a t \%}}{T}+B^{a t \%}
$$

\subsection{Nucleation}

Precipitation in a supersaturated solid solution is associated with the change in molar volume energy and interfacial energy. For a spherical precipitate, it can be expressed by equation 3.8 .

$$
\Delta G^{\theta}(R)=\frac{4}{3} \pi R^{3} \Delta g+4 \pi R^{2} \gamma_{p}
$$

Here, $\Delta g$ is the driving force per mole and $\gamma_{p}$ is the interfacial energy per unit area of the precipitates (see figure 3.1). Precipitation in a system can occur when 


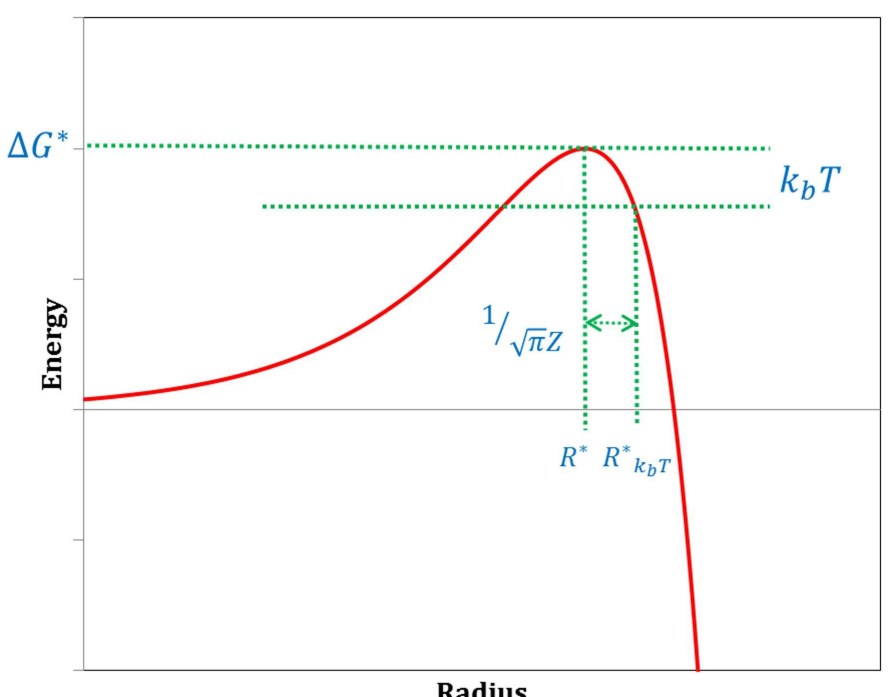

Figure 3.1: A schematic representation of the change in Gibbs energy with precipitate's nucleus radius where the x-axis is in log-scale. $R^{*}$ is the critical radius above which the nucleus is stable. The nucleation energy barrier is $\Delta G^{*}$ and $\mathrm{Z}$ is the Zeldovich factor.

the change in energy $\Delta G$ overcomes a critical value $\Delta G^{*}$. This assumption leads to the minimum stable precipitate nuclei $\left(R>R^{*}\right)$ ( see figure 3.1). The critical radius $R^{*}$ can be expressed as equation 3.9.

$$
R^{*}=-\frac{2 \gamma_{p}}{\Delta G^{*}}=-\frac{R_{\circ}}{S^{P}}
$$

Here, $R_{0}$ is the stable capillary length of the precipitate and expressed as equation 3.10 .

$$
\begin{array}{r}
R_{0}=\frac{2 \gamma_{p} \nu_{a t}^{p}}{k_{b} t} \\
S^{P}=\log \left(\left(\left[X_{A}\right]^{x}\left[X_{B}\right]^{y}\right) / K_{s}\right)
\end{array}
$$

Here, $\nu_{a t}^{p}$ is the mean atomic volume of the precipitate and $S^{P}$ is the supersaturation defined by the solubility product of precipitate.

Becker and Döring [8] and Zeldovich [70] proposed valuable insight into the kinetic features in the expression previously proposed by Volmer and Weber [67]. Considering Becker-Döring and Zeldovich proposition, the nucleation rate can be expressed as equation 3.12 .

$$
J=\beta^{*} N_{0} Z \exp \left[-\frac{\Delta G^{*}}{k_{B} T}\right]
$$


Here, $\beta^{*}$ is the condensation rate of solute atoms in a cluster of radius $R^{*}, N_{0}$ is the number of available nucleation site per unit volume and $Z$ is the Zeldovich factor.

$$
Z=\frac{\nu_{a t}^{p}}{2 \pi R^{* 2}} \sqrt{\frac{\gamma_{p}}{k_{B} T}}
$$

Zeldovich factor can be expressed as equation 3.13. Russel [58] proposition for $\beta^{*}$ takes into account the diffusion coefficient of solute atoms $D_{i}^{m}$, solute atomic fraction in matrix $X_{i}^{m}$, precipitate's constituent atomic fraction $X_{i}^{P}$ and lattice parameter $a$. The expression for $\beta^{*}$ is given in equation 3.14 .

$$
\beta^{*}=\frac{4 \pi R^{* 2}}{a^{4}}\left(\sum_{i} \frac{X_{i}^{P}}{D_{i}^{m} X_{i}^{m}}\right)^{-1}
$$

Kampmann and Wagner [34] proposed expression 3.15 for nucleation rate by introducing incubation time $\tau=4 /\left(2 \pi \beta^{*} Z^{2}\right)$. In their expression, the stable nucleus size $R_{k_{B} T}^{*}$ is defined by equation 3.16 .

$$
\begin{gathered}
\frac{d N}{d t}=N_{0} Z \beta^{*} \exp \left(-\frac{\Delta G^{*}}{k_{b} T}\right) \exp \left(-\frac{\tau}{t}\right) \\
R_{k_{B} T}^{*}=R^{*}+\frac{1}{\sqrt{\pi} Z}
\end{gathered}
$$

Equation 3.15 is true for nucleation at constant temperature and for small precipitate volume fraction $\left(f_{v} \ll 0.1\right)$ that does not affect the available nucleation sites significantly. In a situation, where heterogeneous nucleation is occurring or precipitate volume fraction is large enough $\left(f_{v} \gg 0.1\right)$, nucleation sites are effectively being reduced. In order to take into account the changing number of nucleation sites with volume fraction, a modification of equation 3.15 is required. In the present work, the number of nucleation site is expressed as follows:

$$
N_{0}^{\prime}=N_{0}\left(1-\psi f_{v}\right)
$$

Here, $\psi=\nu_{a t}^{M} / \nu_{a t}^{P}$ is the ratio between the mean atomic volume of matrix and mean atomic volume of precipitate.

\subsection{Gibbs-Thomson effects}

The presence of an interface between the precipitate and the matrix shifts the solubility limit of precipitate. Thus, the interface effect has to be taken into account and it is generally known as Gibbs-Thomson effects [66, 19]. Generalized 
expression [51] of Gibbs-Thomson for the corrected solubility limit of the $A_{x} B_{y}$ precipitate with radius $R$ is given in equation 3.18 .

$$
\frac{2 \gamma_{p} \nu_{a t}^{P}}{R k_{b} T}=\log \left[\frac{X_{A}^{I}(R)}{X_{A}(\infty)}\right]+\log \left[\frac{X_{B}^{I}(R)}{X_{B}(\infty)}\right]
$$

Here, $X_{A}^{I}(R)$ and $X_{B}^{I}(R)$ are the solute $A$ and $B$ mole fractions at the precipitate interface, $X_{A}(\infty)$ and $X_{B}(\infty)$ are the solute $A$ and $B$ mole fractions in the matrix.

Combining the equations $3.7,3.10$ and 3.18 , the solute concentration at the precipitate interface can be expressed as the following equation 3.19.

$$
K_{s} \exp \left[\frac{R_{\circ}}{R}\right]=X_{A}^{I} X_{B}^{I}
$$

\subsection{Precipitate growth or dissolution}

Precipitation can occur both homogeneously and heterogeneously. Depending on the location of the precipitate, it can experience either homogeneous or heterogenous growth. Microstructural properties such as dislocation density, grain size and presence of twin boundaries dictate that one mechanism can dominate the other. Heterogenous precipitation refers to the situation where precipitates nucleate and grow on dislocation or grain boundary. In the present study, precipitate growth lying on dislocation can be assumed negligible as the materials is fully recovered and recrystallized. Therefore, precipitate located on grain boundary are further discussed. In the following, precipitate growth or dissolution in the matrix and grain boundary is discussed in details.

\subsubsection{Growth or dissolution: Precipitate in the matrix}

Precipitate growth rate is controlled by the equilibrium solute concentration at the precipitate-matrix interface. This is defined as the local equilibrium. It is assumed that the solute atoms interaction at the precipitate-matrix interface does not affect the growth. The growth rate can be described by Fick's second law of diffusion (equation 3.20).

$$
\frac{d R}{d t}=\left.\frac{D^{m}}{\psi X^{P}-X^{I}} \frac{\partial X}{\partial r}\right|_{r=R}
$$

Zener proposed a growth rate expression with the assumption of small supersaturation $\left(X^{m}-X^{I}(R) \ll \psi X^{P}-X^{I}\right)$ [71]. Using Zener's hypothesis and introducing Gibbs-Thomson effects, the growth rate equation can be expressed as 
following:

$$
\left\{\begin{array}{l}
\left.\frac{d R}{d t}\right|_{A}=\frac{D_{A}^{m}}{R} \frac{X_{A}^{m}-X_{A}^{I}(R)}{\psi X^{P}-X_{A}^{I}(R)} \\
\left.\frac{d R}{d t}\right|_{B}=\frac{D_{B}^{m}}{R} \frac{X_{B}^{m}-X_{B}^{I}(R)}{\psi X^{P}-X_{B}^{I}(R)} \\
X_{A}^{I} X_{B}^{I}=K_{s} \exp \left[\frac{R_{0}}{R}\right]
\end{array}\right.
$$

Here, $X^{I}$ is the equilibrium atomic concentration of solute atoms at the precipitatematrix interface and $D_{i}^{m}$ refers to solute specie $i$ diffusion rate in the matrix.

In a solid solution, interstitial solute atoms diffusion rate is much faster than the substitutional one $\left(D_{B}^{m} \gg D_{A}^{m}\right)$. Thus the precipitate growth rate is controlled by the substitutional atom's diffusion in the matrix. Considering the fact that the precipitate $\left(A_{x} B_{y}\right)$ growth rate is solely controlled by the diffusion of $A$ solute atoms, the following simplification can be introduced.

$$
X_{B}^{I}(R)=X_{B}^{m} \text { where, } X_{B}^{m} \text { is B interstitial solute atoms in the matrix }
$$

Using the approximation in equation 3.22, precipitate growth rate equation can be solved as there are two equations and two variables $\left(d R / d t\right.$ and $\left.X_{A}^{I}\right)$.

\subsubsection{Growth or dissolution: Precipitate on grain boundary}

Spherical precipitate growing at the grain boundary experiences bulk diffusion of solute atoms and as well as pipe diffusion through the grain boundary. The growth rate depends on the total number of atoms arriving and leaving at the precipitate-matrix interface from the bulk and grain boundary. It is well known that solute atoms have higher diffusivity in the grain boundary than the bulk. Although precipitate's grain boundary intersecting surface area fraction is smaller in comparison with the bulk, it can affect the growth rate considerably. Temperature also determines the dominating mechanism of precipitate growth. Increasing temperature reduces the difference in solute atoms diffusivity in the bulk and grain boundary, thus both the mechanism in parallel can exerts comparable contribution in growth.

Fisher [17] first proposed the thin slab model for grain boundary diffusion. In his hypothesis, grain boundary is assumed to be a thin slab embedded in the matrix having different diffusivity for solute atoms. Fisher's calculation showed that the average grain boundary width, $\delta=0.5 \mathrm{~nm}$, which was indeed confirmed 


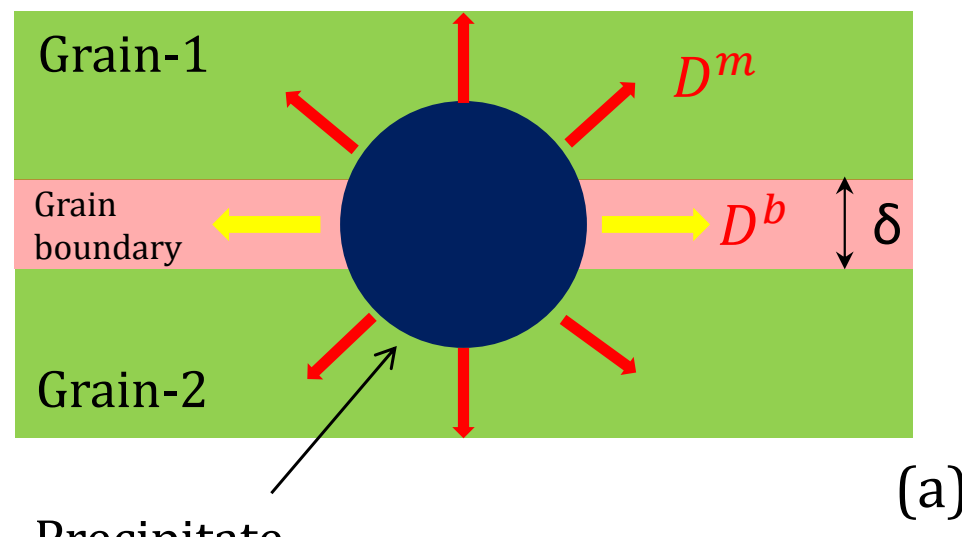

\section{Precipitate}

(a)
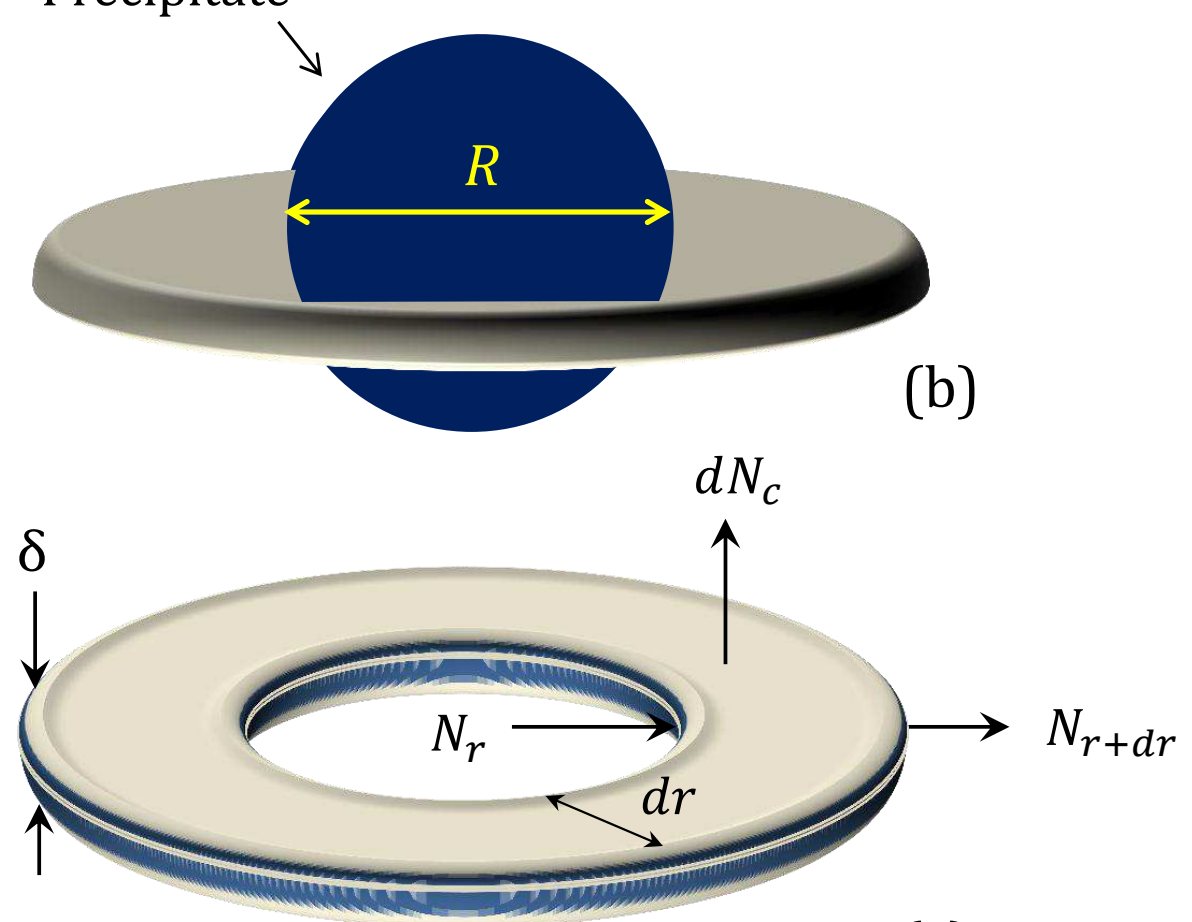

(c)

Figure 3.2: (a) Schematic representation of a precipitate lying at the grain boundary with width, $\delta$. (b) Precipitate lying in a thin slab of grain boundary. (c) A volume element within the grain boundary $[28,10]$. 
by experiments. Following this hypothesis, Hoyt [28] proposed a model where simultaneous effects of bulk and grain boundary solute diffusion are taken into account, assuming zero solute atoms in the grain boundary at the beginning. L. M. Cheng [10] proposed some improvement by considering the pre-existing solute concentration in the grain boundary. In this model, a grain boundary precipitate is considered to be spherical with a thin slab of material attached along its equator [28, 10], as illustrated in figure 3.2(b). In the present situation where both bulk and grain boundary diffusion is occurring simultaneously, concentration profile from the precipitate interface to the boundary can be approximated as a summation of the two coexisting profiles. One solution consists of the bulk diffusion and the other one for the grain boundary only.

Concentration profile within the grain boundary can be determined by analyzing the volume element presented in figure 3.2(c). In the figure, the number of solute atoms entering and leaving the volume element per unit time and unit area at points $r$ and $r+d r$ are $N_{r}$ and $N_{r+d r} . d N_{c}$ is the number of atoms diffusing to the grain boundary from the bulk per unit time. $d N_{b}$ is the change in number of solute atoms in the grain boundary due to diffusion. The conservation of solute atoms within the volume element can be expressed as expression 3.23.

$$
N_{r}-N_{r+d r}-d N_{c}=d N_{b}
$$

Let us assume, that the solute concentration within the grain boundary is $C_{b}=$ $C_{b}(r, t), C_{m}$ is the mean solute concentration in the bulk, $D^{m}$ is the diffusion coefficient of solute atom in the matrix, $D^{b}$ is the diffusion coefficient in the grain boundary and $D_{n}$ is the mean grain diameter. Here, $r$ is the radius of the ring of grain boundary around the precipitate.

$$
\begin{gathered}
N_{r}=-\left.D^{b}(2 \pi r \delta) \frac{\partial C_{b}}{\partial r}\right|_{r} \\
d N_{b}=2 \pi r d r \delta \frac{\partial C_{b}}{\partial t} \\
d N_{c}=h 2 \pi r d r\left(C_{b}-C_{m}\right)
\end{gathered}
$$

Here, $d N_{c}$ is an approximation over the number of solute atoms convecting between grain boundary and bulk. The convection coefficient $h$ can be approximated for grain boundary as $h=4 D^{m} / D_{n}$ [31].

Using equation 3.24 to 3.26 , the rate of change in solute concentration within 
the grain boundary can be obtained as equation 3.27.

$$
\frac{\partial C_{b}}{\partial t}=D^{b}\left(\frac{\partial^{2} C_{b}}{\partial r^{2}}+\frac{1}{r} \frac{\partial C_{b}}{\partial r}-\frac{h}{\delta D^{b}}\left(C_{b}-C_{m}\right)\right)
$$

For a stationary solute concentration in the grain boundary, $\partial C_{b} / \partial t=0$ and assuming that $C=C_{b}-C_{m}$ and $\beta^{2}=h /\left(\delta D^{b}\right)$ then the equation 3.27 transforms to:

$$
r^{2} \frac{\partial^{2} C}{\partial r^{2}}+r \frac{\partial C}{\partial r}-\beta^{2} r^{2} C=0
$$

The boundary conditions can be expressed as 3.29 and 3.30. Here, $R$ is the precipitate radius and $C^{I}$ is interfacial solute concentration.

$$
\begin{gathered}
C_{b}=C_{m} \text { when } r \gg R \\
\text { and } \\
C_{b}=C^{I} \text { when } r=R
\end{gathered}
$$

Equation 3.28 is the modified form of Bessel function [2] and the general form of solution can be written as 3.31.

$$
C_{b}(r)=A_{1} I_{0}(\beta r)+A_{2} K_{0}(\beta r)
$$

In equation 3.31, $A_{1}$ and $A_{2}$ are constants and $I_{0}$ and $K_{0}$ are modified bessel functions for the first and second kind of order zero. One of the boundary conditions suggest that $C_{b}$ will tend to zero when $r$ reaches infinity. As $I_{0}$ reaches infinity as $r$ reaches infinity [2], $A_{1}$ must be zero. Equation 3.31 can be reduced to much simpler form by applying the the boundary conditions 3.29 and 3.30 .

$$
C_{b}(r)=\frac{C^{I}-C_{m}}{K_{0}(\beta R)} K_{0}(\beta r)+C_{m}
$$

$K_{0}(\beta r)$ can be approximated as [2]:

$$
K_{0}(\beta r) \simeq \sqrt{\frac{\pi}{2(\beta r)}} e^{-(\beta r)}
$$

The growth rate of precipitate lying at the grain boundary can be found by a solute balance over the surface of the particle. The mass balance can be expressed as 3.34 .

$$
4 \pi R^{2} C^{I} d R+\left.D^{m} \frac{\partial C_{m}}{\partial r}\right|_{R} 4 \pi R^{2} d t+\left.D^{b} \frac{\partial C_{b}}{\partial r}\right|_{R} 2 \pi R \delta d t=4 \pi R^{2} C_{p} d R
$$


Combining equation 3.32 to 3.34 we can obtain the equation for precipitate growth rate of radius $R$. Here, it is assumed that the solute concentration $C$ is equal to the mole fraction $X / \nu_{a t}$ and $\psi=\nu_{a t}^{M} / \nu_{a t}^{P}$ is the ratio between the mean atomic volume of matrix and mean atomic volume of precipitate. Here, $\nu_{a t}$ is the mean atomic volume. The precipitate growth rate equation can finally be expressed as follows:

$$
\frac{d R_{b}}{d t}=\frac{X^{m}-X^{I}}{\psi X^{P}-X^{I}}\left(\frac{D^{m}}{R}+\frac{\delta D^{b}}{4 R^{2}}+\frac{\delta D^{b} \beta}{2 R}\right)
$$

The same expression as classical homogeneous growth rate expression can be deduced from equation 3.41, if we replace grain boundary width $\delta=0$.

\subsubsection{Number of precipitate lying on the grain boundary}

In this section, partitioning between the number of precipitate that lies at the grain boundary and in the matrix is discussed. Multi precipitate class consideration required assumption while allocating number of precipitates from a class $(i)$ to the bulk and grain boundaries. In this study, it is assumed that particular number of precipitate from each class $i$ of the distribution can be found both at the grain boundaries and the rest in the matrix. So, the assumption refers that all precipitate radii that is present in the distribution can be found in the grain boundaries. Following this assumption, number density of precipitate that can be found in the grain boundaries is calculated using Zener's approximation [71].

The total grain boundary area of a system having a mean grain size $D_{n}$ and the total number of grains $N_{g b}$ per unit volume can be expressed as follows:

$$
A_{g b}=\frac{1}{2} \lambda_{n} D_{n}^{2} N_{g b}
$$

Here, $\lambda_{n}$ is a geometrical factor describing the grain boundary shape, for spherical grain $\lambda_{n}$ is $4 \pi$.

The total number of grains $N_{g b}$ per unit volume:

$$
N_{g b}=\frac{1}{\lambda_{v} D_{n}^{3}}
$$

Here, $\lambda_{v}$ is another geometrical factor describing the volume of a grain in three dimension and for a spherical grain $\lambda_{v}$ is $4 \pi / 3$.

Zener [71] proposed that the number of precipitates from class $i$ interacting with per unit area of grain boundary can be expressed as follows:

$$
N_{i}^{b}=2 r_{i} N_{i}
$$




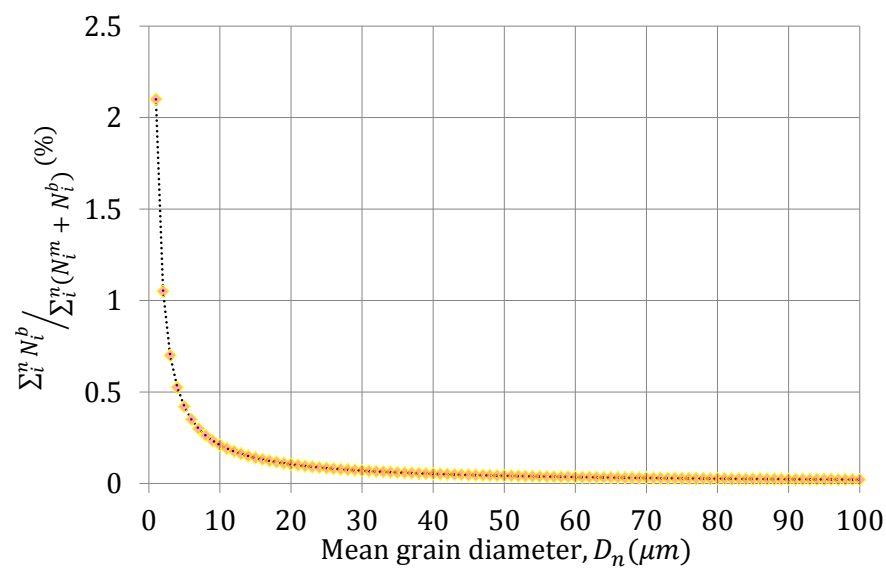

Figure 3.3: Comparison between number of precipitate lying in the matrix and grain boundaries in relation with mean grain size $\left(D_{n}\right)$.

From equation 3.36 to 3.38 , number of precipitate from class $i$ lying at the grain boundary can be found as follows:

$$
N_{i}^{b}=\frac{\lambda_{n}}{\lambda_{v} D_{n}} N_{i} r_{i}
$$

Similarly the total number of precipitates that are embedded in the matrix can be written as:

$$
\sum_{i}^{n} N_{i}^{m}=\sum_{i}^{n} N_{i}-\sum_{i}^{n} N_{i}^{b}
$$

In figure 3.3, the ratio between the total number of precipitates that can lie at the grain boundary $\left(\sum_{i}^{n} N_{i}^{b}\right)$ and the total number precipitates $\left(\sum_{i}^{n}\left(N_{i}^{m}+N_{i}^{b}\right)\right)$ is presented. It can be seen from the figure 3.3, that for mean grain sizes $D_{n}<4 \mu \mathrm{m}$, more than $0.5 \%$ of the total precipitate population can be found at the grain boundaries. On the other hand, mean mean sizes $D_{n}>4 \mu \mathrm{m}$ yield less than $0.5 \%$ of the total precipitate than can be found on the grain boundary. This small number fraction of the grain boundary precipitate suggests rather a negligible contribution to the overall precipitation kinetics when $D_{n}>4 \mu \mathrm{m}$.

\subsubsection{Comparison between grain boundary and bulk precip- itate growth or dissolution rate}

A comparison between precipitate growth or dissolution rate at the grain boundary $\left(d R_{b} / d t\right)$ and matrix $(d R / d t)$ is presented in the following discussion. The expressions for precipitate growth rate in grain boundaries can be summarized as 
Table 3.1: List of bulk and grain boundary diffusion data of $\mathrm{Al}$ in austenite.

\begin{tabular}{ccc}
\hline Data type & Value & Reference \\
\hline Al in Austenite, ${ }^{0} D_{A l}^{m}$ & $8.1 \times 10^{-3}$ & {$[59]$} \\
Al in Austenite, $Q_{A l}^{m}$ & $252 \mathrm{~kJ} / \mathrm{mol}$ & {$[59]$} \\
$\mathrm{Al}$ in grain boundary, ${ }^{0} D_{A l}^{b}$ & $3 \times 10^{-4}$ & {$[36]$} \\
$\mathrm{Al}$ in grain boundary, $Q_{A l}^{b}$ & $167.4 \mathrm{~kJ} / \mathrm{mol}$ & {$[36]$} \\
\hline
\end{tabular}

follows:

$$
\begin{gathered}
\frac{d R_{b}}{d t}=k\left(\frac{D^{m}}{R}+\frac{\delta D^{b}}{4 R^{2}}+\frac{\delta D^{b} \beta}{2 R}\right) \\
\text { where, } \\
\beta=\sqrt{\frac{h}{\delta D^{b}}} \\
h=\frac{4 D^{m}}{D_{n}} \\
k=\frac{X^{m}-X^{I}}{\psi X^{P}-X^{I}}
\end{gathered}
$$

The expression for precipitate growth rate in the bulk is as follow:

$$
\frac{d R}{d t}=k \frac{D^{m}}{R}
$$

Here, $D^{m}$ is the solute diffusion rate in the matrix, $D^{b}$ is the solute diffusion rate in the grain boundary, $\delta$ is the grain boundary width having a value of $0.5 \mathrm{~nm}$ [17], $X^{I}$ is the solute mole fraction at the precipitate-matrix interface, $X^{m}$ is the solute mole fraction in the matrix, $X^{P}$ is the mole fraction in the precipitate and $\psi=$ $\nu_{a t}^{M} / \nu_{a t}^{P}$ is the ratio between the mean atomic volume of matrix and the mean atomic volume of precipitate.

Bulk and grain boundary diffusion data of $\mathrm{Al}$ in austenite is given in table 3.1. In this calculation, mean austenite grain diameter $D_{n}$ is taken as $10 \mu \mathrm{m}$.

In figure 3.4, a comparison between AlN precipitate growth rate in grain boundary and in the matrix is presented. From the figure, it can be seen that with increasing precipitate radius, difference between the grain boundary and the bulk growth rate decreases. Precipitate radius larger than $80 \mathrm{~nm}$ has 8 times growth (dissolution) rate at the grain boundary than the bulk. Besides that, increase in temperature also decreases in the ratio $\left(d R_{b} / d R\right)$. So, it can be said from figure 3.4, that at higher temperatures $(>1100 K)$ precipitate growth (dissolution) due to grain boundary diffusion and bulk diffusion of solute atoms is equally contributing factor. In the case of, smaller precipitate $(<5 \mathrm{~nm})$ lying at the grain boundary, solute 


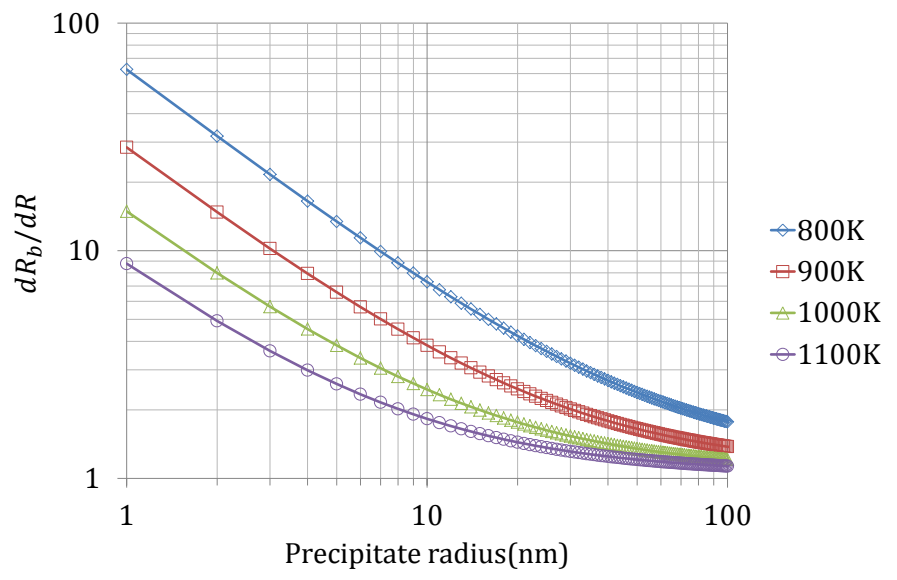

Figure 3.4: Comparison between $\mathrm{AlN}$ precipitate growth rate in grain boundary $\left(\frac{d R_{b}}{d t}\right)$ and matrix $\left(\frac{d R}{d t}\right)$ for different precipitate radiuses and temperatures. Here, mean grain diameter $D_{n}$ is $10 \mu \mathrm{m}$.

diffusion in the grain boundary is the dominating factor controlling the growth or dissolution.

\subsection{Mass balance}

Precipitate growth or dissolution changes the solute concentration in the matrix. So, it is essential to calculate the amount of solute atoms remaining after each time step of precipitate growth or dissolution. This is generally done by mass balance of solute atoms in the matrix and in the precipitate.

Let us consider a matrix with different precipitate species $\left(f_{j}\right.$ is volume fraction of species $j$ ). The mean atomic volume of the matrix and mean atomic volume of the precipitate species are expressed as $\nu_{m}^{a t}$ and $\nu_{p_{j}}^{a t}$. Parameter $\psi_{j}$ is defined as $\psi_{j}=\nu_{m}^{a t} / \nu_{p_{j}}^{a t}$. The atomic fraction of each chemical species $i$ is known and expressed as $X_{0}^{i}$.

The conservation of mass states that element $i$ is either in the matrix or in the precipitate:

$$
n_{0}^{i}=n_{m}^{i}+\sum_{j} n_{p_{j}}^{i}
$$

Here, $n_{0}^{i}$ is the total number of atoms, $n_{m}^{i}$ is the number of solute atoms in the matrix and $n_{p_{j}}^{i}$ is the number of solute atoms in the precipitate $j$. Dividing the equation 3.46 by the total number of atoms $n_{T}$ leads to:

$$
X_{0}^{i}=\frac{n_{m}^{i}}{n_{m}} \frac{n_{m}}{n_{T}}+\sum_{j} \frac{n_{p_{j}}^{i}}{n_{p_{j}}} \frac{n_{p_{j}}}{n_{T}}
$$


The number of atoms $n_{p_{j}}$ in the precipitate $j$ can be expressed as a function of $\nu_{p_{j}}^{a t}:$

$$
n_{p_{j}}=\frac{\nu_{p_{j}}}{\nu_{p_{j}}^{a t}}=\nu_{T} \frac{\nu_{p_{j}}}{\nu_{T} \nu_{p_{j}}^{a t}}=\nu_{T} \frac{f_{j}}{\nu_{p_{j}}^{a t}}=\psi_{j} f_{j} \frac{\nu_{T}}{\nu_{m}^{a t}}
$$

Similarly, the number of atoms in the matrix can be expressed as follows:

$$
n_{m}=\frac{\nu_{m}}{\nu_{m}^{a t}}=\frac{\nu_{T}-\sum_{j} \nu_{p_{j}}}{\nu_{m}^{a t}}=\frac{\nu_{T}}{\nu_{m}^{a t}}\left[1-\sum_{j} f_{j}\right]
$$

The combination of equation 3.46 to 3.49 , leads to the expression of mass balance:

$$
X^{i}=\frac{X_{0}^{i}\left[1+\sum_{j} \psi_{j} f_{j}-\sum_{j} f_{j}\right]-\sum_{i} X_{p}^{i} \psi_{j} f_{j}}{1-\sum_{j} f_{j}}
$$

\subsection{Implementation}

In this work, precipitation modeling is done by the implementation of classical nucleation and growth theories (CNGTs). "Lagrange-like" multi-class approach is chosen [43]. Precipitate size distribution (PSD) is discretely divided into several classes, the mean radius and volume fraction evolution is calculated as a function of time [52]. Each class of precipitate is treated separately and individual class radii evolution with time, collectively gives the overall size distribution evolution.

The corner point pinning concept is presented in the abnormal grain growth modeling (see chapter 2). Here the largest precipitates lie in the corner points of the austenite grain boundary. Besides that, as already discussed, particular number fraction (see section 3.5.2.1) of all precipitate classes as a function of their radii reside at the grain boundary. So, the corner point precipitates and precipitate contributing to Zener pinning will experience heterogeneous growth or dissolution. For simplicity in the current approach, the austenite grain boundary is assumed immobile. Thus precipitates that lie at the grain boundary or corner point will experience heterogenous growth or dissolution throughout the simulation time. The same is true for precipitate lying in the matrix. It is also assumed for simplicity that the precipitate nucleation occurs only homogeneously.

The calculation procedure for homogeneous and heterogenous precipitate nucleation and growth is presented in figure 3.5. Precipitate with largest radius will fill up the number of corner points $\left(N^{*}\right)$ available and the rest will lie in the matrix or grain boundaries. In a system with more than one precipitate type, the probability of individual precipitate type to lie in the corner points is solely depends on the 


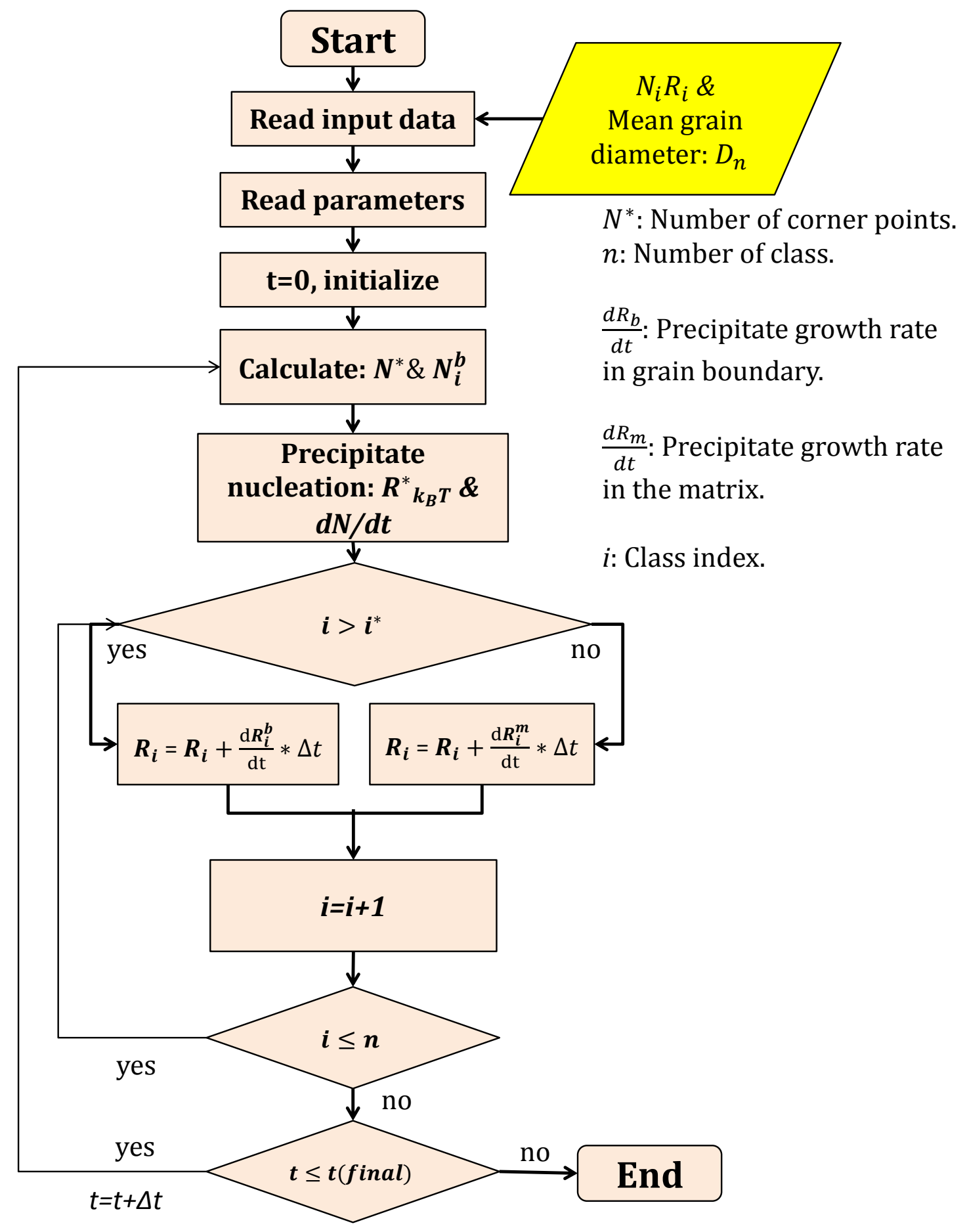

Figure 3.5: A schematic representation of the precipitation model implementation including both homogeneous and heterogeneous growth of precipitates. 
radii of the class and total number density of all precipitate type.

At each time step, the precipitate radius evolution and number of precipitate in a new class is calculated using following the equations 3.51, 3.52 and 3.53.

$$
\begin{array}{r}
R_{i}(t+\Delta t)=R_{i}(t)+\frac{d R_{i}^{m}}{d t} \Delta t, \text { Precipitate in matrix } \\
R_{i}(t+\Delta t)=R_{i}(t)+\frac{d R_{i}^{b}}{d t} \Delta t, \text { Precipitate in grain boundary }
\end{array}
$$

New class is added to the pre-existing $n$ number of classes, if the nucleation rate is positive.

$$
\begin{gathered}
N_{n+1}=\frac{d N}{d t} \times \Delta t \\
R_{n+1}=R_{k_{b} T}^{*}
\end{gathered}
$$

\subsection{Effects of model parameters}

In this section, the effects of different model parameters on possible outcome of the precipitation model prediction are discussed. The present precipitation model takes into account couple of physical parameters. Among them, precipitate solubility product, diffusion coefficient and precipitate interfacial energy effects are detailed below for homogeneous precipitate nucleation and growth.

Mean austenite grain size plays an important role in determining the number of precipitate that experience heterogeneous (grain boundary and triple point precipitate) and homogeneous growth. Efforts are given in understanding the effects of mixed growth (homogeneous and heterogeneous) mechanism on precipitation kinetics for different mean austenite grain sized system.

\subsubsection{Effects of solubility product}

The precipitate solubility product is expressed in terms of two constants $A$ and $B$ in the form $\log \left(K_{s}\right)=-A / T+B$. In figure 3.6, effects of precipitate solubility product is studied by varying $B$. Figure 3.6 suggest that with decreasing value of $B$, unstable AlN precipitate transforms to a stable one. This is evident as for $B=-3.5$, as AlN volume fraction increased at $900 K$. Thus decrease in the value of $B$ can be associated with the decrease in solubility of precipitate in the matrix and vise versa. 

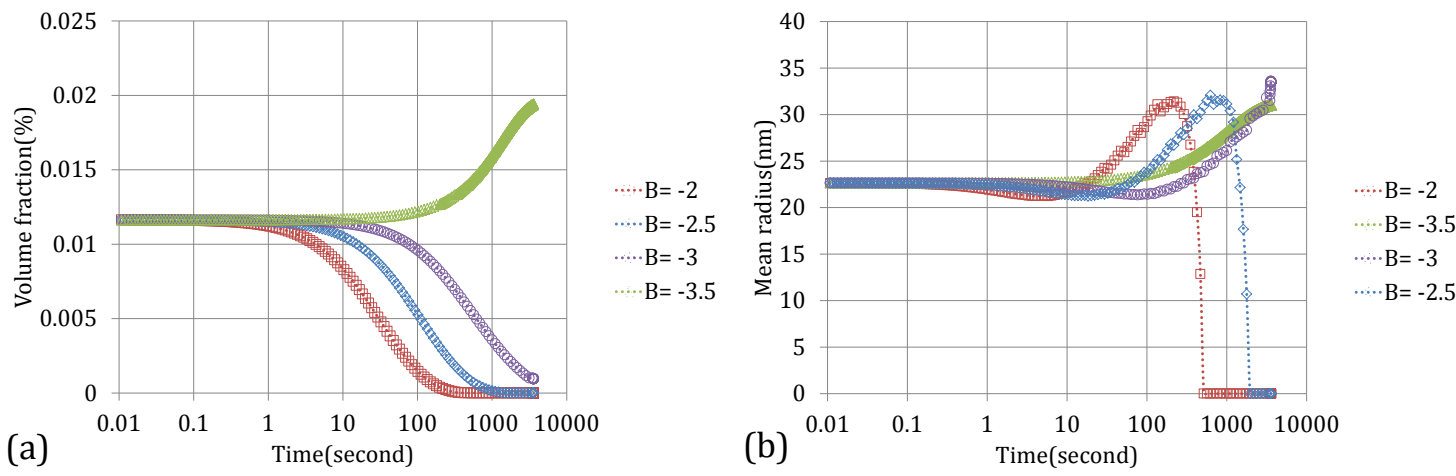

Figure 3.6: Effects of solubility product $(B)$ on AlN volume fraction and mean radius evolution. Here, $\log \left(K_{s}\right)=-3793.6 / T+B$ and temperature is $\mathrm{T}=900^{\circ} \mathrm{C}$.
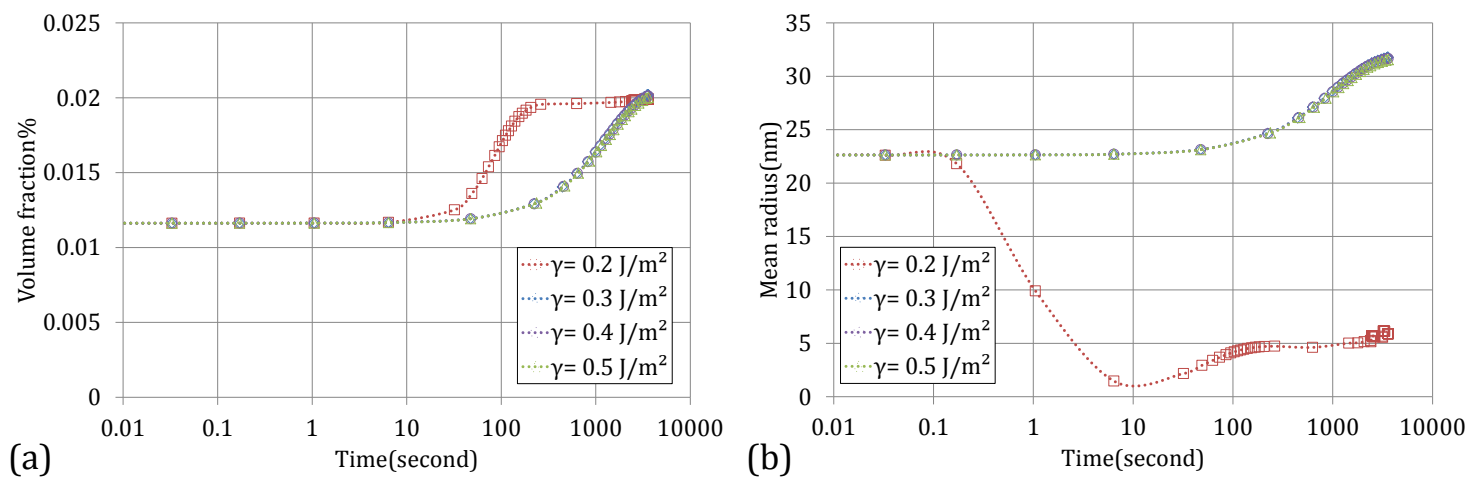

Figure 3.7: Effects of precipitate-matrix interfacial energy $\left(\gamma_{p}\right)$ on AlN volume fraction and mean radius evolution. Here, solubility product is $\log \left(K_{s}\right)=$ $-3793.6 / T-3.5137$ and temperature is $\mathrm{T}=900^{\circ} \mathrm{C}$. 

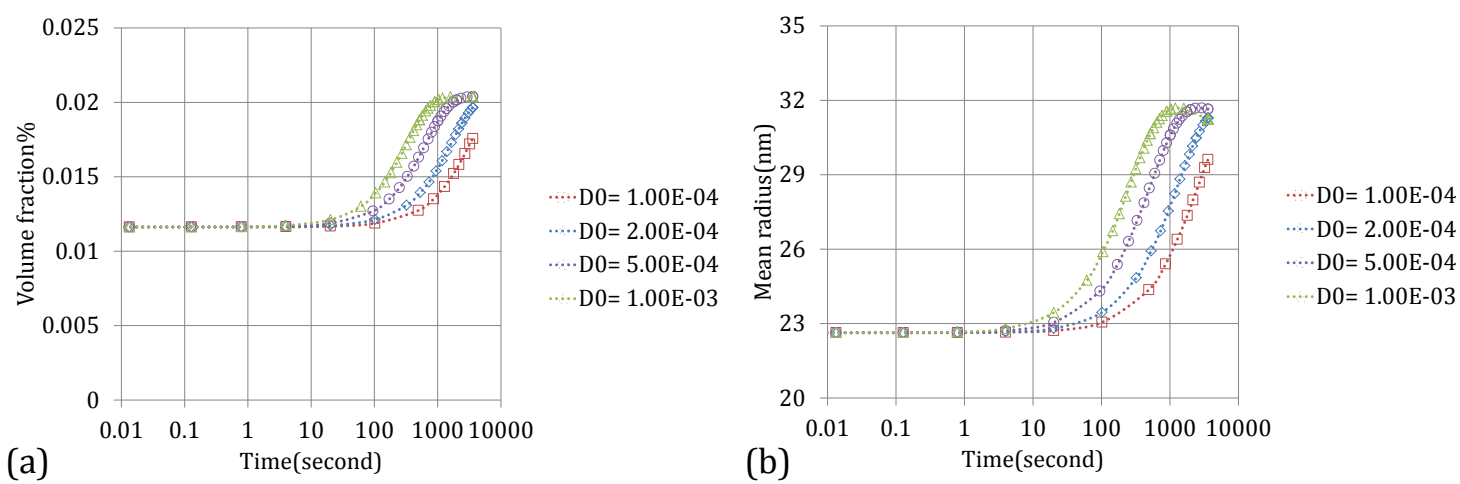

Figure 3.8: Effects of diffusion coefficient $\left(D_{0}\right)$ on AlN volume fraction and mean radius evolution. Here, solubility product is $\log \left(K_{s}\right)=-3793.6 / T-3.5137, \mathrm{Al}$ diffusion activation energy $Q=253.4 \mathrm{kj}$ and temperature is $\mathrm{T}={ }^{\circ} \mathrm{C}$.

\subsubsection{Effects of precipitate interfacial energy}

In figure 3.7, the effects of precipitate interfacial energy $\left(\gamma_{p}\right)$ on AlN volume fraction and mean radius evolution are presented. From figure 3.7, it can be seen that for $\gamma_{p}=0.2$ the volume fraction evolution shows faster kinetics and the equilibrium reached at $\sim 130 \mathrm{sec}$. AlN mean radius rather shows a decrease because nucleation of stable smaller precipitates are possible with lower interfacial energy. On the other hand, for all the other values $\left(\gamma_{p}>0.2\right)$ of interfacial energy both volume fraction and mean radius show quite similar kinetics. Therefore, interfacial energy does not affect precipitation equilibrium, it changes AlN mean radius evolution kinetics when $\gamma_{p} \leq 0.2$.

\subsubsection{Effects of diffusion coefficient}

In figure 3.8, $\mathrm{Al}$ diffusion coefficient $\left(D_{0}\right)$ effects on the $\mathrm{AlN}$ precipitate kinetics is presented. An increase in diffusion coefficient effectively increases the diffusion rate of $\mathrm{Al}$ in the matrix. This is why, the precipitate volume fraction reaches the equilibrium quantity faster for higher values of diffusion coefficient. A similar effect can be seen in the AlN mean radius evolution. Thus, the choice of diffusion coefficient can affect the precipitation kinetics prediction.

\subsubsection{Effects of mean austenite grain size $\left(D_{n}\right)$ on precipitate growth}

In figure 3.9, effects of mean austenite grain size $\left(D_{n}\right)$ on AlN precipitate kinetics is presented in the case of dissolution and also growth. Both homogeneous and heterogeneous precipitate growth mechanisms are considered in the calculation. 

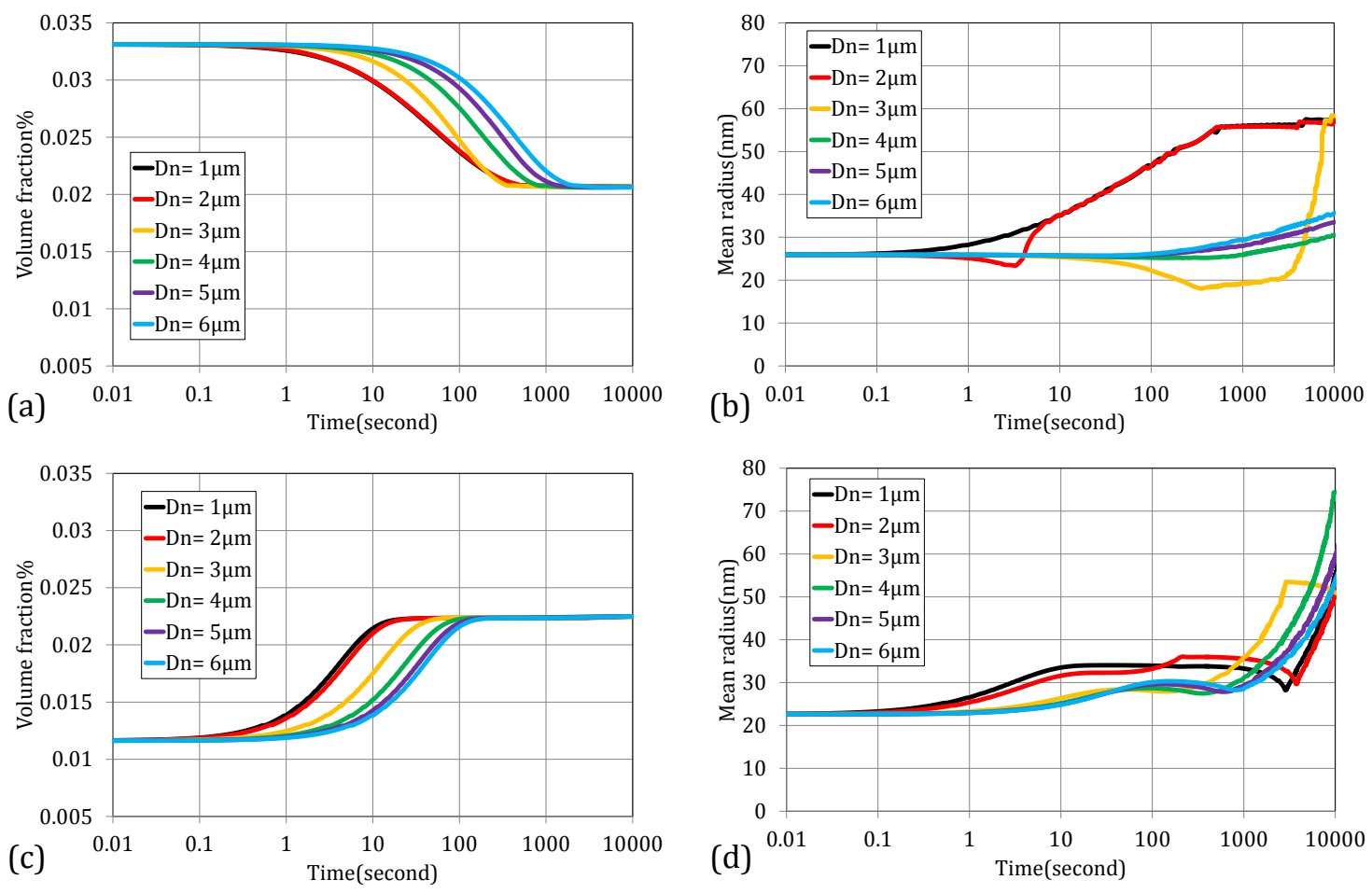

Figure 3.9: Effects of mean austenite grain diameter on AlN. Evolution of a dissolving state: (a) volume fraction and (b) mean radius evolution. Evolution of a growing state: (c) volume fraction and (d) mean radius evolution. Here, solubility product is $\log \left(K_{s}\right)=-3793.6 / T-3.5137$ and temperature is $\mathrm{T}=1050^{\circ} \mathrm{C}$. 
Precipitate experiencing homogenous and heterogenous growth is determine using the assumptions presented in section 3.5.2.1. Figure 3.9 (a) and (c) suggests that mean austenite grain size has no effect on the equilibrium volume fraction. Systems with smaller $D_{n} \leq 3 \mu \mathrm{m}$ show faster dissolution and growth in comparison with the larger $D_{n}>3 \mu \mathrm{m}$.

From figure 3.9 (b) and (d) it can be observed that system with smaller mean austenite grain size yield larger AlN mean radius. According to equation 3.39, the number of precipitates lying at the grain boundary and corner point per unit volume is depends on the mean grain diameter $\left(D_{n}\right)$. So, in a system with comparatively smaller mean grain sizes, higher number of precipitates can be situated at the grain boundaries and corner points. Precipitates located in those location experience accelerated growth or dissolution due to higher diffusivity of solute atoms. Higher number fraction of AlN precipitate experiencing accelerated growth results in larger AlN mean radius.

It is also noticeable from figure 3.9 (b) and (d), that the evolution of AlN precipitate's mean radius is considerably faster when $D_{n}=1$ to $3 \mu \mathrm{m}$. Grain sizes larger than $3 \mu \mathrm{m}$ show quite similar kinetics of both AlN mean radius and volume fraction. Thus systems with larger than $3 \mu \mathrm{m}$ of mean austenite grain size has relatively small effects of heterogeneous precipitate growth. So, in a system having mean austenite grain size larger than $3 \mu \mathrm{m}$ will yield similar outcome in precipitation kinetics where only homogeneous or mixed (homogeneous and heterogeneous) growth or dissolution mechanism is considered.

\subsection{Model Applications}

In this section, the precipitation model is applied to two industrial alloys. Discussion is presented by comparing the experimentally determined precipitation states and the model predictions.

\subsubsection{Materials and heat treatment}

The precipitation state evolution at different temperatures is studied in two industrial low alloy steels: (1) steel-A and (2) steel-B. The compositions of these alloys are presented in table 3.2 and 3.3, where the major precipitating elements are highlighted.

In steel-A, the major precipitating element is $\mathrm{Al}$. On the other hand, steel-B has both $\mathrm{Nb}$ and $\mathrm{Al}$ as precipitating elements but $\mathrm{Nb}$ has 3 times less atomic $\%$ in comparison with the $\mathrm{Al}$.

Two initial states of steel-A are chosen to study the precipitation state. The heat 
Table 3.2: Composition of industrial steel-A alloy in atomic \%

\begin{tabular}{cccccccccc}
\hline $\mathrm{C}$ & $\mathrm{Si}$ & $\mathrm{Mn}$ & $\mathrm{Ni}$ & $\mathrm{Cr}$ & $\mathrm{V}$ & $\mathrm{Ti}$ & $\mathrm{Al}$ & $\mathrm{Nb}$ & $\mathrm{N}$ \\
0.702 & 0.407 & 0.455 & 1.22 & 1.47 & 0.00696 & 0.00194 & 0.0549 & 0.00171 & 0.0598 \\
\hline
\end{tabular}

Table 3.3: Composition of steel-B in atomic \%

\begin{tabular}{cccccccc}
\hline $\mathrm{C}$ & $\mathrm{Si}$ & $\mathrm{Mn}$ & $\mathrm{Ni}$ & $\mathrm{Cr}$ & $\mathrm{Al}$ & $\mathrm{Nb}$ & $\mathrm{N}$ \\
0.0963 & 0.352 & 1.22 & 0.133 & 1.23 & 0.0701 & 0.0221 & 0.0929 \\
\hline
\end{tabular}
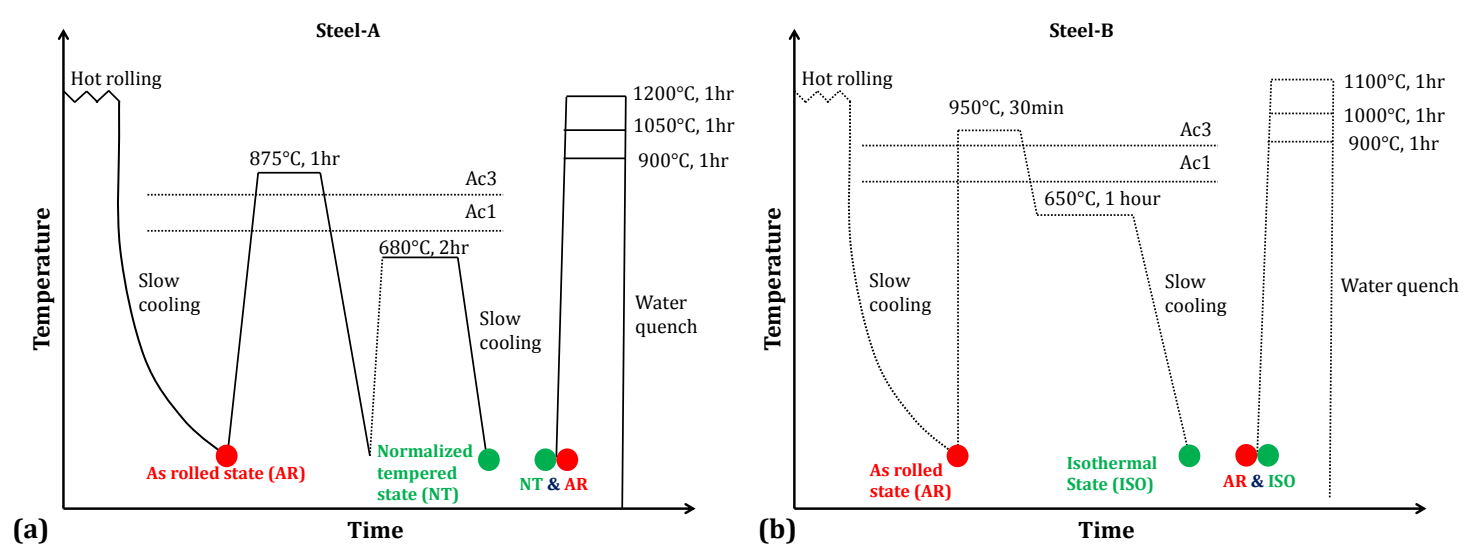

Figure 3.10: (a) Heat treatment cycle of steel-A to obtain As Rolled (AR) and Normalized Tempered (NT). (b) Heat treatment cycle of steel-B to obtain As Rolled (AR) and Isothermally heat treated State (ISO). In both cases, heat treatment cycles to obtain the initial state and the subsequent heat treatment temperatures are shown. 


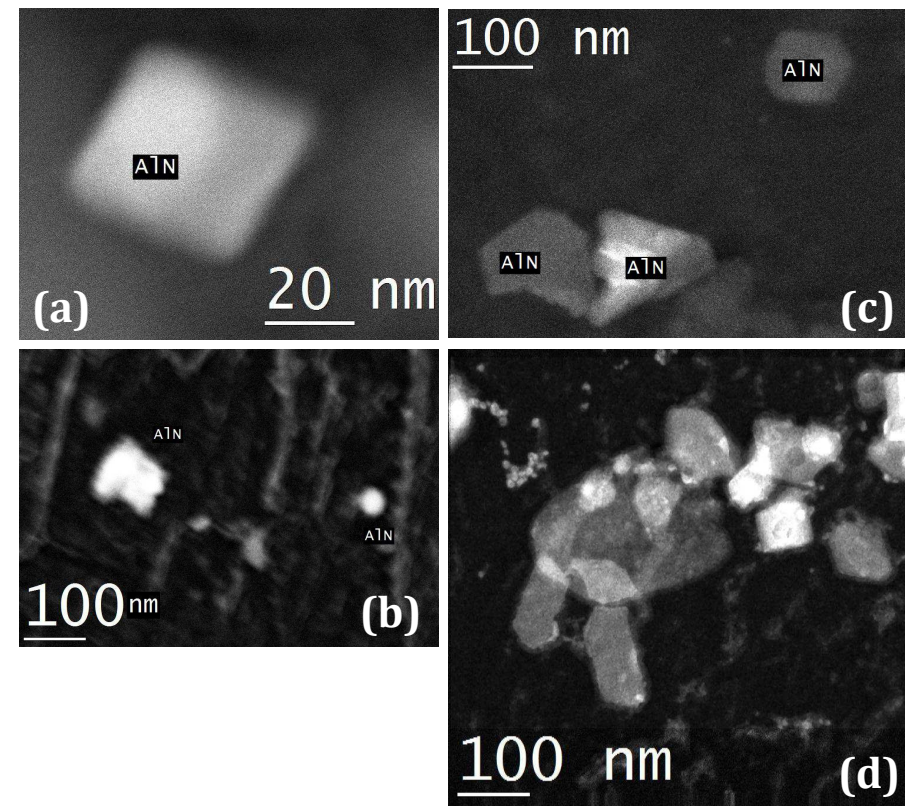

Figure 3.11: High Angle Annular Dark Filed (HAADF) images of precipitate in steel-A: (a) AR state heat treated at $875^{\circ} \mathrm{C}$ for 20 min including ramp time, (b) AR state heat treated at $1050^{\circ} \mathrm{C}$ for $1 \mathrm{hr}$, (c) $\mathrm{NT}$ state heat treated at $875^{\circ} \mathrm{C}$ for $20 \mathrm{~min}$ and (d) $\mathrm{NT}$ state heat treated at $1050^{\circ} \mathrm{C}$ for $1 \mathrm{hr}$.

treatment cycles are shown in figure 3.10(a). The as rolled state (AR) which has a bainitic microstructure is obtained by rolling the cast section from the billet. The rolling cycle was terminated at $>800^{\circ} \mathrm{C}$ and then air cooled. Normalized Tempered state $(\mathrm{NT})$ is obtained by isothermal heat treatment of the AR state above $\left(A C_{3}\right)$ and below $\left(A C_{1}\right)$ the austenite transformation temperatures. It should be mentioned that the NT state is produced to maximum the precipitate volume fraction. Both AR and NT states are subsequently heat treated at different temperatures to study the effects of different austenitizing temperatures on precipitation. In figure 3.11, AlN precipitate's Transmission Electron Microscope (TEM) micrographs of both the AR and NT state heat treated at 875 and $1050^{\circ} \mathrm{C}$ are presented. It can be seen from figure 3.11 that in all states, the dominating precipitate is AlN.

As in steel-A, two initial states are chosen from the steel-B. The as rolled (AR) state is obtained by rolling followed by slow air cooling, while another one is obtained by isothermal heat treatment above $\left(A C_{3}\right)$ and below $\left(A C_{1}\right)$ the austenite transformation temperatures (see figure 3.10(b)). Slow cooling followed by the rolling ensured a fully recrystallized microstructure. Isothermal heat treatment of the AR state is done in order to obtain a fully precipitated state. Subsequent heat treatments are performed on the AR and ISO states to study precipitation behavior at different austenitizing temperatures (shown in figure 3.10(b)). 


\subsubsection{Precipitate solubility products}

In the present study, solubility product of precipitates in steel-A and steel$\mathrm{B}$ is experimentally measured. Precipitates chemistry are confirmed using ICP measurement and Transmission Electron Microscope (TEM) on carbon extracted replica. ICP analysis gives the precipitates volume fraction in samples heat treated at different temperatures in terms of constitutive substitutional element's in Parts Per Million (PPM) (for details see appendix A). The PPM value can be converted to precipitate volume fraction using expression 3.55.

$$
f_{v}^{A_{x} B_{y}}(T, t)=f_{m}^{A} \frac{\rho_{F e}}{\rho_{A_{x} B_{y}}}\left(\frac{y}{x} \frac{M_{B}}{M_{A}}+1\right)
$$

Here, $f_{v}^{A_{x} B_{y}}(T, t)$ is volume fraction of $A_{x} B_{y}$ precipitate, $f_{m}^{A}$ is the mass fraction of element $A$ measured using ICP, $\rho_{F e}$ and $\rho_{A_{x} B_{y}}$ are molecular density of the matrix and the precipitate and $M_{A}$ and $M_{B}$ are molar mass of the substitutional and interstitial elements.

$$
X^{i}=\frac{X_{0}^{i}\left[1+\sum_{j} \psi_{j} f_{j}-\sum_{j} f_{j}\right]-\sum_{i} X_{p}^{i} \psi_{j} f_{j}}{1-\sum_{j} f_{j}}
$$

At a particular temperature, the solute concentration in the solid solution can be calculated using expression 3.56. The calculated solute concentration in solid solution is used in equation 3.7 to obtain the solubility product $\left(K_{S}\right)$ of precipitate in the alloys.

\subsubsection{AlN solubility}

In figure 3.12, AlN solubility products from literatures [41, 16, 50, 22, 26, 25, $15,61,11,35]$ are presented together with the one measured in the present work. It can be seen that, there is a considerable scatter in the literature data range. This is understandable from the fact that the AlN solubility is measured in different alloy compositions. Furthermore, the solubility products are measured using different heat treatment cycles varying in both holding time and previous thermal history. There is also a difference between AlN solubility product in the steel-A and steel-B. The reason can be again correlated to the difference in the alloys composition.

\subsubsection{NbC solubility}

In figure 3.13, NbC solubility product in steel-B is presented in comparison with the one that can be found in the literatures $[32,38,46,48,29,27,47,7,7]$. It is assumed that in steel-B, Nb is precipitated with $\mathrm{C}$ as stoichiometric NbC. The stoichiometric composition assumption simplified the model calculation. It can be 


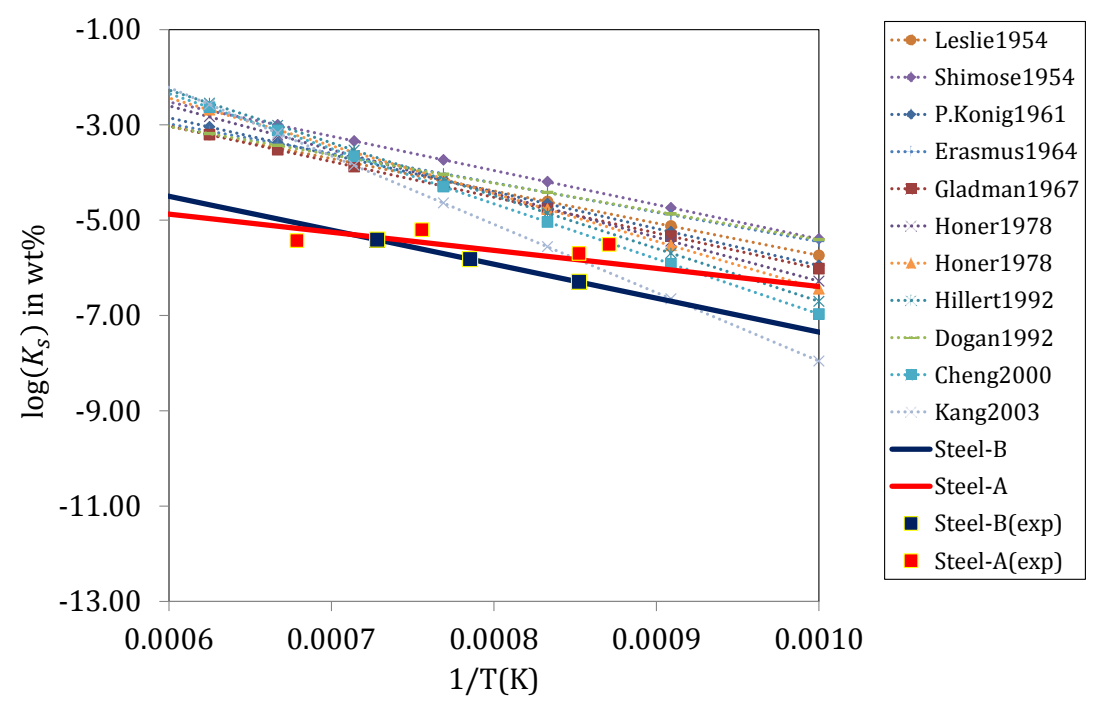

Figure 3.12: Comparison between AlN solubility product in steel-A and steel-B with the literatures in austenite $(\gamma)$.

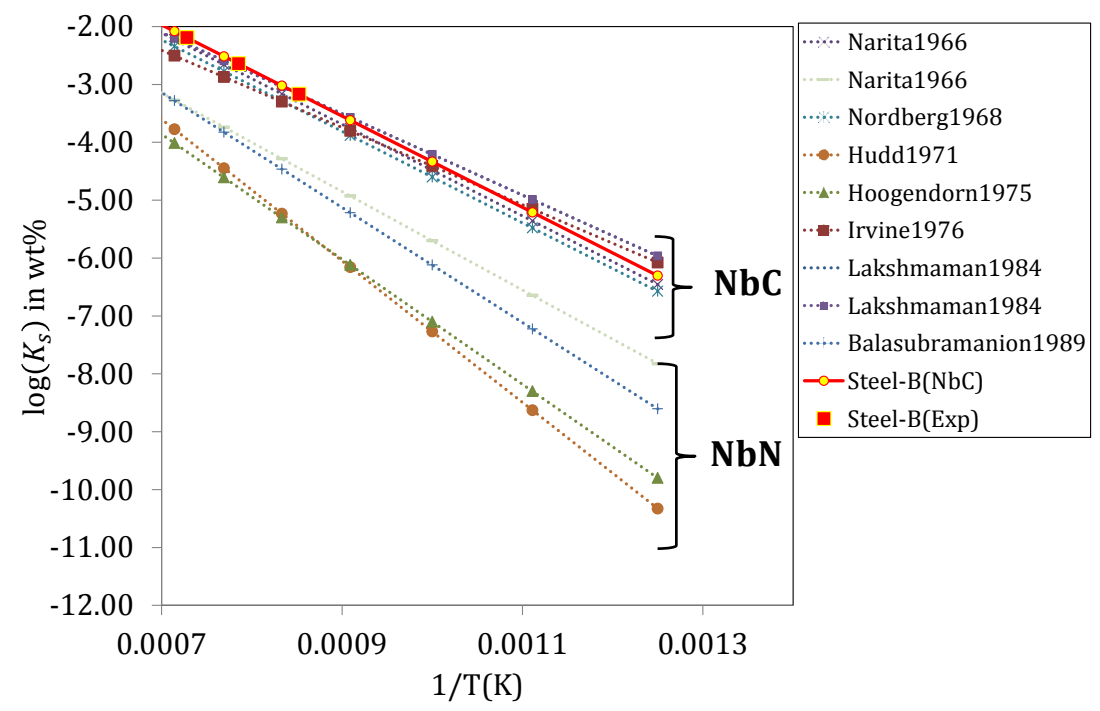

Figure 3.13: Solubility product of $\mathrm{NbC} / \mathrm{NbN}$ in austenite $(\gamma)$ Iron. Comparison between $\mathrm{NbC}$ solubility product in steel-B with published data. 

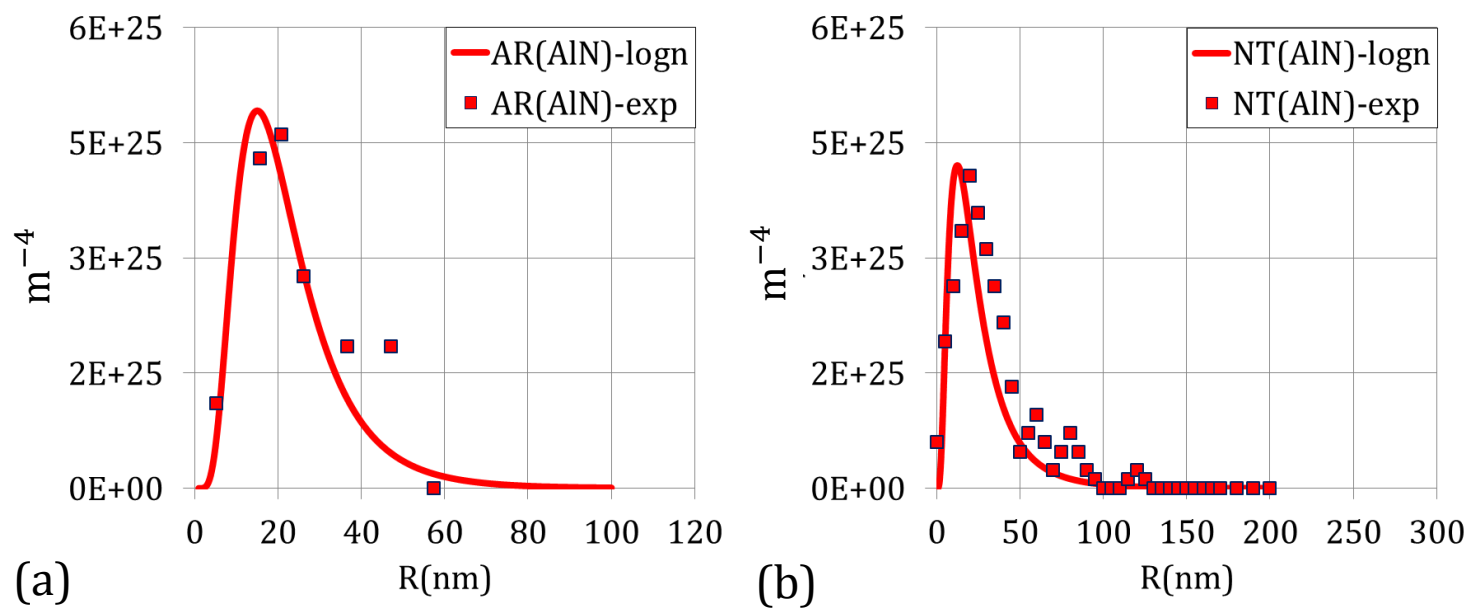

Figure 3.14: Steel-A's initial AlN precipitate size distribution (a) As Rolled (AR) state and (b) Normalized Tempered (NT) state. Here, $\mathrm{m}^{-4}$ is determined by dividing the precipitate number density $\mathrm{m}^{-3}$ by the class width $\Delta R$.

observed that the measured solubility product is quite similar to the NbC solubility product reported in literatures.

\subsubsection{Precipitate size distribution}

Precipitate size distribution is experimentally measured using carbon extraction replica. The replica is analyzed in Transmission Electron Microscope (TEM) using the High Angled Annular Dark Field (HAADF) mode and EDS analysis. Image processing software is used to measure the equivalent precipitate radius (see appendix A). The measured radiuses of precipitate are used to build the precipitate size distribution by classifying them into several classes. Number density of the precipitate size distribution is obtained by normalizing the size distribution's frequency to the experimentally determined volume fraction $\left(f_{v}\right)$.

Figure 3.14 representing the AlN precipitate size distribution in AR and NT state of steel-A. AR and NT state's AlN size distribution of 1000 particles assembly is taken as input parameter in the precipitation model to simulate the subsequent heat treatment effect. Here, the 1000 particles class assembly are determined from the lognormal fit of the experimentally measured distribution.

In figure 3.15, AlN and NbC precipitates size distribution in AR and ISO states of steel-B is presented. In order to simulate the subsequent heat treatments of both AR and ISO state, similarly initial size distribution of 1000 particles class assemble is taken as input parameter in the precipitation model. 

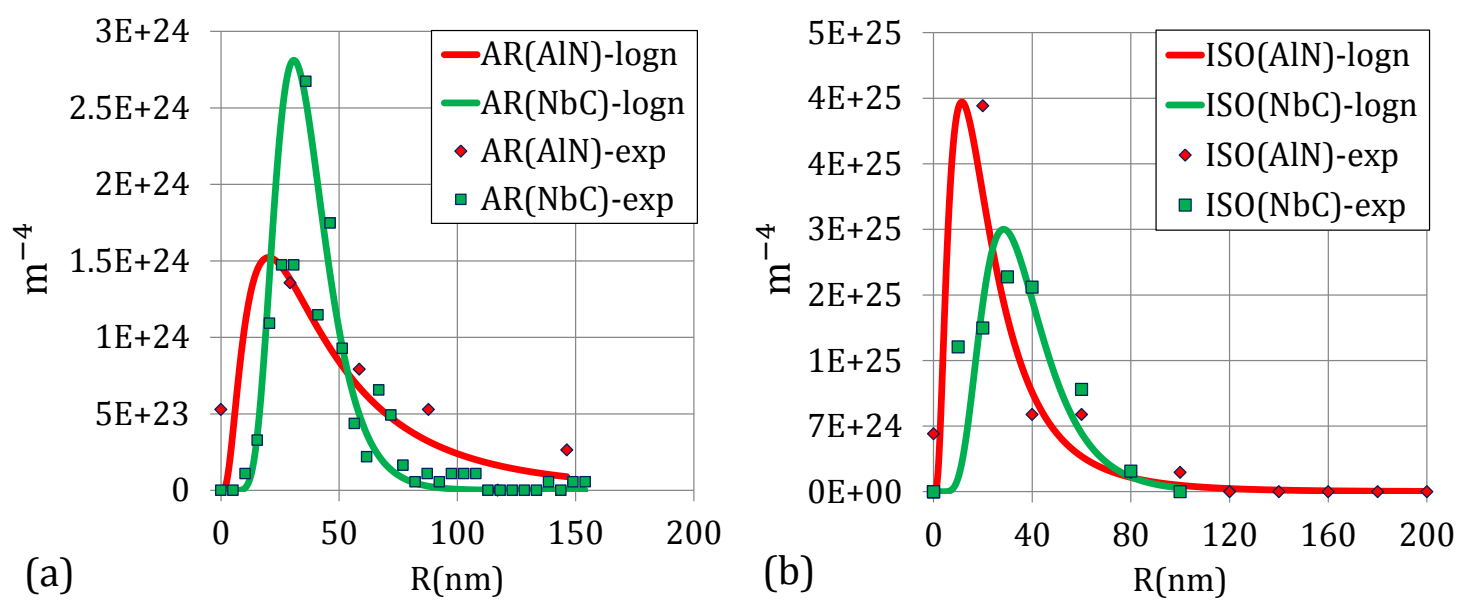

Figure 3.15: Steel-B's initial AlN and NbC precipitates size distribution (a) As Rolled (AR) state and (b) Isothermally heat treated (ISO) state. Here, $\mathrm{m}^{-4}$ is determined by dividing the precipitate number density $\mathrm{m}^{-3}$ by the class width $\Delta R$.

\subsubsection{Model parameters}

The model parameters are listed in table 3.4. Diffusion coefficients found in the literatures consist of a large scatter. Among all the available data, diffusion coefficients of $\mathrm{Al}, \mathrm{Nb}, \mathrm{C}$ and $\mathrm{N}$ are chosen which represent the currently studied systems in comparison with the experimental observations. The Nb grain boundary diffusion coefficient can not be found in the literature. So, from Fridberg's [18] work it is approximated that the $\mathrm{Nb}$ diffusion activation energy in grain boundary, $Q_{N b}^{b}=Q_{N b}^{m} / 1.8$.

The solubility products of both $\mathrm{AlN}$ and $\mathrm{NbC}$ are determined using regular solution model presented in the section 3.2. Precipitates interfacial energy data are approximated using experimentally determined precipitates mean radius evolution with time and temperature. Moreover it has already been shown that precipitate interfacial energy has negligible effects on the thermodynamic equilibrium and kinetics of evolution of precipitate.

In table 3.5, experimentally determined initial austenite grain diameter of steelA and steel-B is listed. It can be seen that for both steel-A and steel-B, the fully precipitated (NT and ISO) state has smaller mean austenite grain diameter than the As Rolled (AR) state. Moreover, the mean grain sizes are larger than $3 \mu \mathrm{m}$ and as stated earlier, heterogeneous precipitate growth or dissolution will have negligible effects on the overall precipitation kinetics. 
Table 3.4: List of input parameters for precipitation model.

\begin{tabular}{ccc}
\hline Data type & Value & Reference \\
\hline AlN crystal structure & Cubic & {$[60]$} \\
AlN lattice parameter & $0.407 \mathrm{~nm}$ & {$[60]$} \\
AlN crystal structure & Wurtzite & {$[60]$} \\
AlN lattice parameter & $a=0.311 \mathrm{~nm}$ & {$[60]$} \\
& $\& c / a=1.6$ & \\
AlN interfacial energy & $0.5 \mathrm{~J} / \mathrm{m}^{2}$ & Present work \\
Al in Austenite, ${ }^{0} D_{A l}^{m}$ & $8.1 \times 10^{-3}$ & {$[59]$} \\
Al in Austenite, $Q_{A l}^{m}$ & $252 \mathrm{~kJ} / \mathrm{mol}$ & {$[59]$} \\
Al in grain boundary, ${ }^{0} D_{A l}^{b}$ & $3 \times 10^{-4}$ & {$[36]$} \\
Al in grain boundary, $Q_{A l}^{b}$ & $167.4 \mathrm{~kJ} / \mathrm{mol}$ & {$[36]$} \\
$\mathrm{N}$ in Austenite, ${ }^{0} D_{N}^{m}$ & $9.1 \times 10^{-5}$ & {$[21]$} \\
$\mathrm{N}$ in Austenite, $Q_{N}^{m}$ & $168.6 \mathrm{~kJ} / \mathrm{mol}$ & {$[21]$} \\
\hline $\mathrm{NbC}$ crystal structure & $\mathrm{Cubic}$ & {$[55]$} \\
$\mathrm{NbC}$ lattice parameter & $0.44686 \mathrm{~nm}$ & {$[55]$} \\
$\mathrm{NbC}$ interfacial energy & $0.5 \mathrm{~J} / \mathrm{m}^{2}$ & Present work \\
$\mathrm{Nb}$ in Austenite, ${ }^{0} D_{N b}^{m}$ & $8.3 \times 10^{-5}$ & {$[21]$} \\
$\mathrm{Nb}$ in Austenite, $Q_{N b}^{m}$ & $266.5 \mathrm{~kJ} / \mathrm{mol}$ & {$[21]$} \\
$\mathrm{Nb}$ in grain boundary, ${ }^{0} D_{N b}^{b}$ & $8.3 \times 10^{-5}$ & {$[18]$} \\
$\mathrm{Nb}$ in grain boundary, $Q_{N b}^{b}$ & $Q_{N b}^{m} / 1.8 \mathrm{~kJ} / \mathrm{mol}$ & {$[18]$} \\
$\mathrm{C}$ in Austenite, ${ }^{0} D_{C}^{m}$ & $0.1 \times 10^{-4}$ & {$[21]$} \\
$\mathrm{C}$ in Austenite, $Q_{C}^{m}$ & $135.7 \mathrm{~kJ} / \mathrm{mol}$ & {$[21]$} \\
\hline steel-B: $\log \left(K_{s}^{\gamma}(A l N)\right)($ at fraction $)$ & $-7130.7 / \mathrm{T}-1.1352$ & Present work \\
steel-B:log $\left(K_{s}^{\gamma}(N b C)\right)($ at fraction $)$ & $-7880.1 / \mathrm{T}-0.9003$ & Present work \\
\hline steel-A: $\log \left(K_{s}^{\gamma}(A l N)\right)($ at fraction $)$ & $-3793.6 / \mathrm{T}-3.5137$ & Present work \\
\hline
\end{tabular}

Table 3.5: Initial austenite mean grain diameter of steel-A and steel-B.

\begin{tabular}{c|c|c}
\hline Steel-A & AR state, $D_{n}=16 \mu \mathrm{m}$ & NT state, $D_{n}=10 \mu \mathrm{m}$ \\
\hline Steel-B & AR state, $D_{n}=7 \mu \mathrm{m}$ & ISO state, $D_{n}=6 \mu \mathrm{m}$ \\
\hline
\end{tabular}




\subsubsection{Precipitate volume fraction evolution}

In this section, a comparison between precipitation model predictions considering homogeneous precipitate growth and experimental measurements is presented. It is worth mentioning here that in both steel-A and steel-B, austenite mean grain diameter is $\geq 5 \mu \mathrm{m}$ (see table 3.5). In section 3.8.4, it is already shown that when mean grain diameter is larger than $3 \mu \mathrm{m}$, heterogeneous precipitate growth or dissolution has negligible effect on precipitation kinetics. For this reason, it is reasonable to assume that the precipitate nucleation and growth or dissolution is occurring homogeneously.

Precipitate volume fraction evolution at different temperatures are modeled for different states of the alloys. For example, in steel-A both the AR (As Rolled) and NT (Normalize Tempered) and in steel-B both the AR (As Rolled) and ISO (Isothermally Treated) states are modeled. The model and experimental results are comparatively presented and arguments are offered to explain agreement and disagreement between them.

\subsubsection{Industrial alloy: steel-A}

In figure 3.16(a), AlN volume fraction evolution at different temperatures in the steel-A's AR state is shown. It can be seen that in the AR state, at $875^{\circ} \mathrm{C}$ and $900^{\circ} \mathrm{C}$ the AlN volume fraction increases. Model predictions for $875^{\circ} \mathrm{C}$ and $900^{\circ} \mathrm{C}$ heat treatment shows, that the equilibrium AlN volume fraction is not reached after 3600 sec. At $1200^{\circ} \mathrm{C}$, complete dissolution of AlN occurs after 20 sec into the heat treatment. A comparison between the experimental measurements and model predictions rather shows a reasonable agreement considering the experimental errors. It should be mentioned here that the AlN solubility product is calculated using the volume fraction measured in NT state, heat treated at different temperatures for $1 \mathrm{hr}$.

In figure 3.16(b), AlN volume fraction evolution at different temperatures in the steel-A's NT state is shown. At $875^{\circ} \mathrm{C}$ and $900^{\circ} \mathrm{C}$ there is a small decrease in precipitate volume fraction. Model predictions suggest that at $1200^{\circ} \mathrm{C}$, complete AlN dissolution occurs in $200 \mathrm{sec}$. Comparison between the experimental measurements and model predictions show quite good agreement for the three heat treatment temperatures presented.

Figure 3.17 represents AlN volume fraction evolution in both the AR and NT state at $1050^{\circ} \mathrm{C}$. In the AR state, we can observed an increase in AlN volume fraction while in the NT state it decreases. In both states, the model predicts that the equilibrium reached in $\sim 200 \mathrm{sec}$. The NT state before heat treatment at $1050^{\circ} \mathrm{C}$ is fully precipitated by heat treatment at $875^{\circ} \mathrm{C}$ for $2 \mathrm{hr}$. At $1050^{\circ} \mathrm{C}$ temperature, 

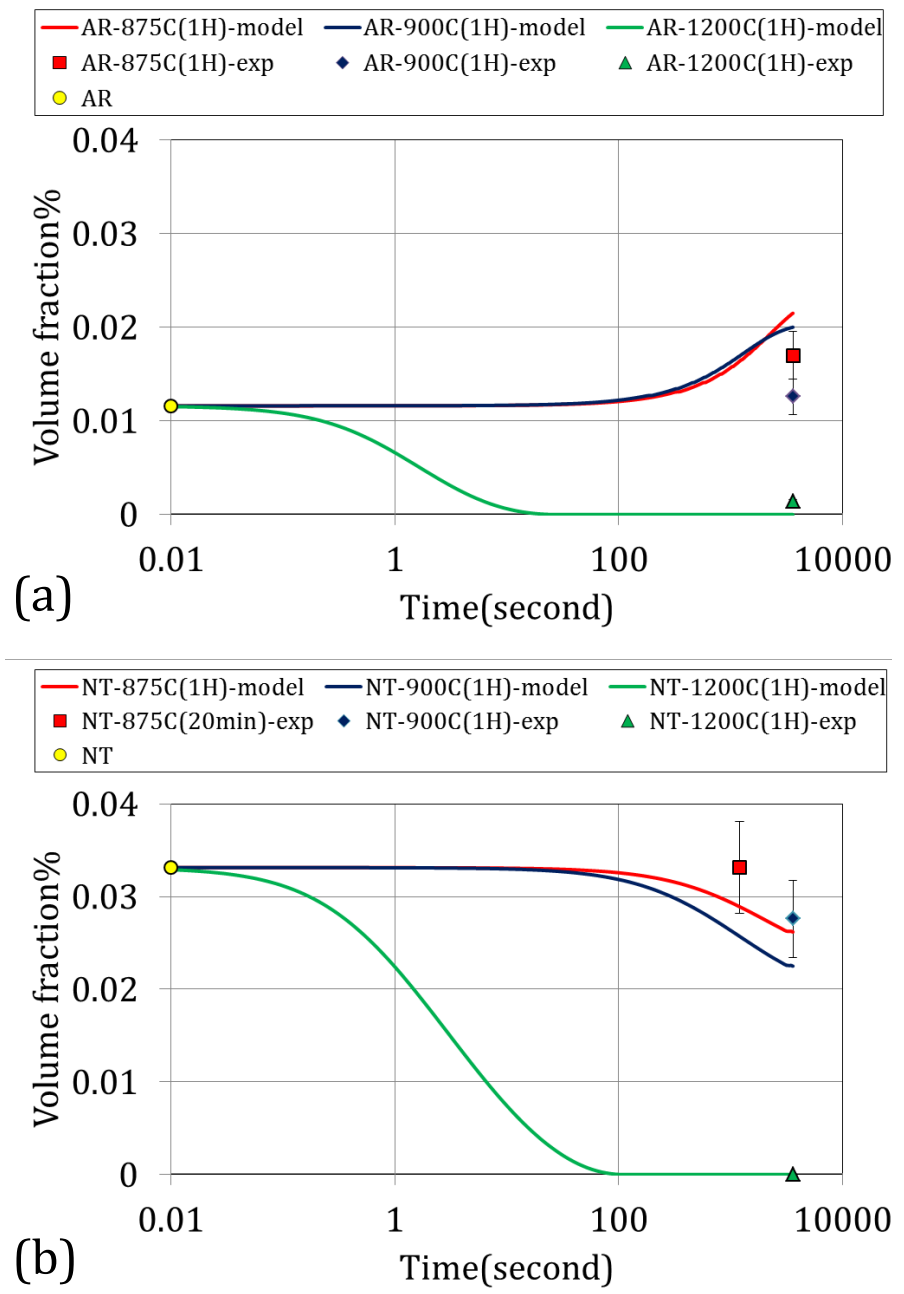

Figure 3.16: Precipitate volume fraction evolution in steel-A (a) AR state at $875^{\circ} \mathrm{C}$, $900^{\circ} \mathrm{C}$ and $1200^{\circ} \mathrm{C}(\mathrm{b}) \mathrm{NT}$ state at $875^{\circ} \mathrm{C}, 900^{\circ} \mathrm{C}$ and $1200^{\circ} \mathrm{C}$.

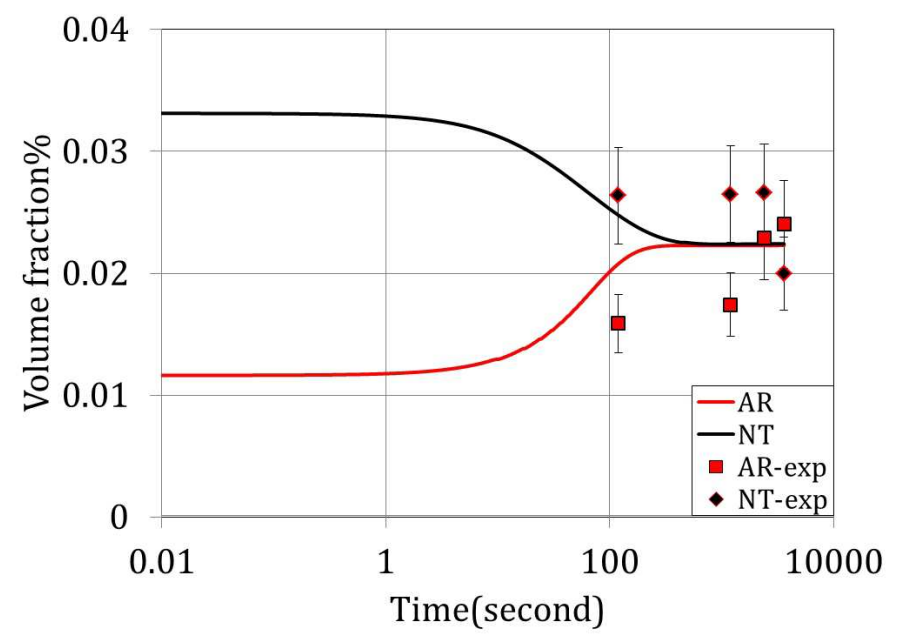

Figure 3.17: Precipitate volume fraction evolution in steel-A's AR and NT state at $1050^{\circ} \mathrm{C}$. 

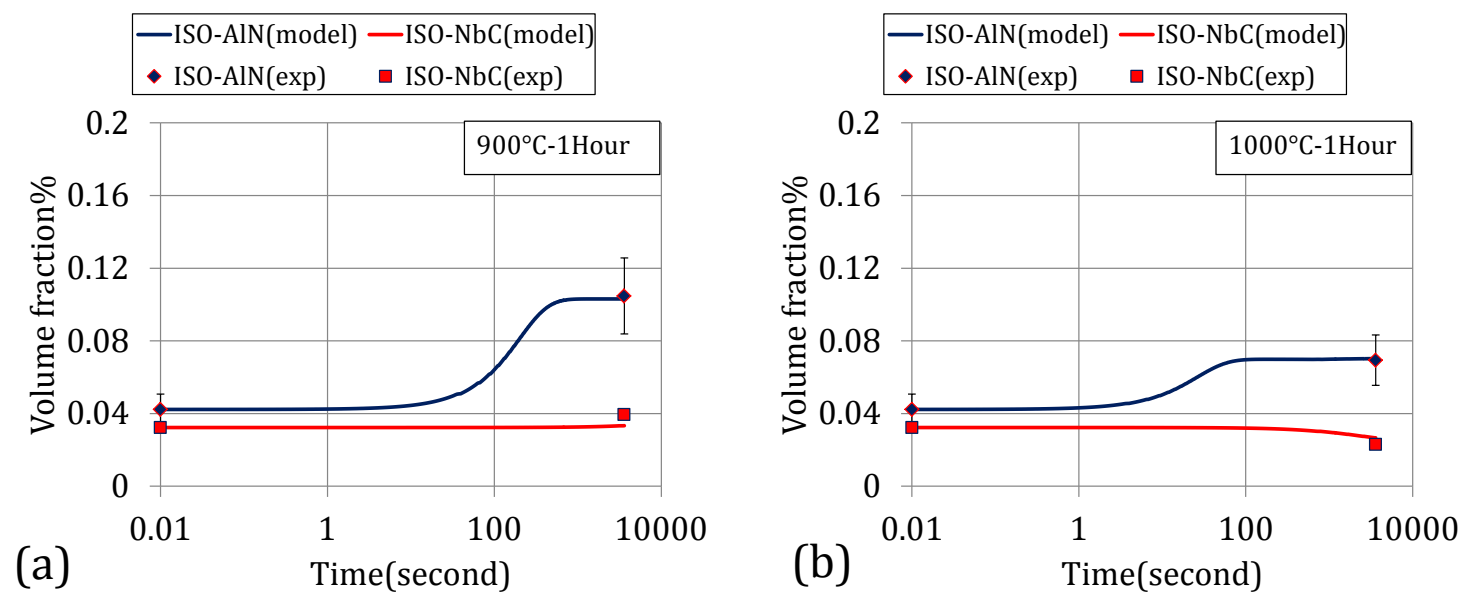

Figure 3.18: Precipitate volume fraction evolution in steel-B's ISO state (a) $900^{\circ} \mathrm{C}$ for $1 \mathrm{hr}$ and (b) $1000^{\circ} \mathrm{C}$ for $1 \mathrm{hr}$.

AlN has lower equilibrium volume fraction in the austenite matrix. Due to lower equilibrium volume fraction at $1050^{\circ} \mathrm{C}$ in $\mathrm{NT}$ state, the volume fraction decreases. On the other hand, the AR state has free solute $\mathrm{Al}$ and $\mathrm{N}$ atoms in the matrix. The solute atoms can contribute to the AlN precipitates coarsening at $1050^{\circ} \mathrm{C}$. Thus, there is an increase in volume fraction in the AR state.

\subsubsection{Industrial alloy: steel-B}

In figure 3.18, a comparison between model predictions and experimental measurements are shown, where concurrent evolution of AlN and NbC precipitate in steel-B's ISO state are presented. At $900^{\circ} \mathrm{C}$ (see Figure $3.18(\mathrm{a})$ ) AlN volume fraction increases while $\mathrm{NbC}$ volume fraction remains quite unchanged. On the other hand, heat treatment at $1000^{\circ} \mathrm{C}$ shows a smaller increase in AlN volume fraction in comparison with $900^{\circ} \mathrm{C}$. At this temperature, the $\mathrm{NbC}$ volume fraction shows a slight decrease.

The extreme right hand side volume fraction value in figure 3.18 is correlated to the solubility product of the precipitates at that particular temperature. At $900^{\circ} \mathrm{C}$, the equilibrium AlN volume fraction is reached at $580 \mathrm{sec}$ and after that the volume fraction remains unchanged. At $1000^{\circ} \mathrm{C}$, the model predicts that the equilibrium AlN volume fraction is reached at 80 sec. Higher temperature offers faster diffusion of $\mathrm{Al}$ solute atoms. Because of the faster diffusion, the equilibrium at $1000^{\circ} \mathrm{C}$ is reached earlier than $900^{\circ} \mathrm{C}$. Finally it can be said, from the presented comparison that the model prediction is quite in agreement with the experimental data.

In figure 3.19, comparison between model predictions and experimental results are shown for both AR and ISO states. At $1100^{\circ} \mathrm{C}$ (see figure $3.19(\mathrm{a})$ ) in the 

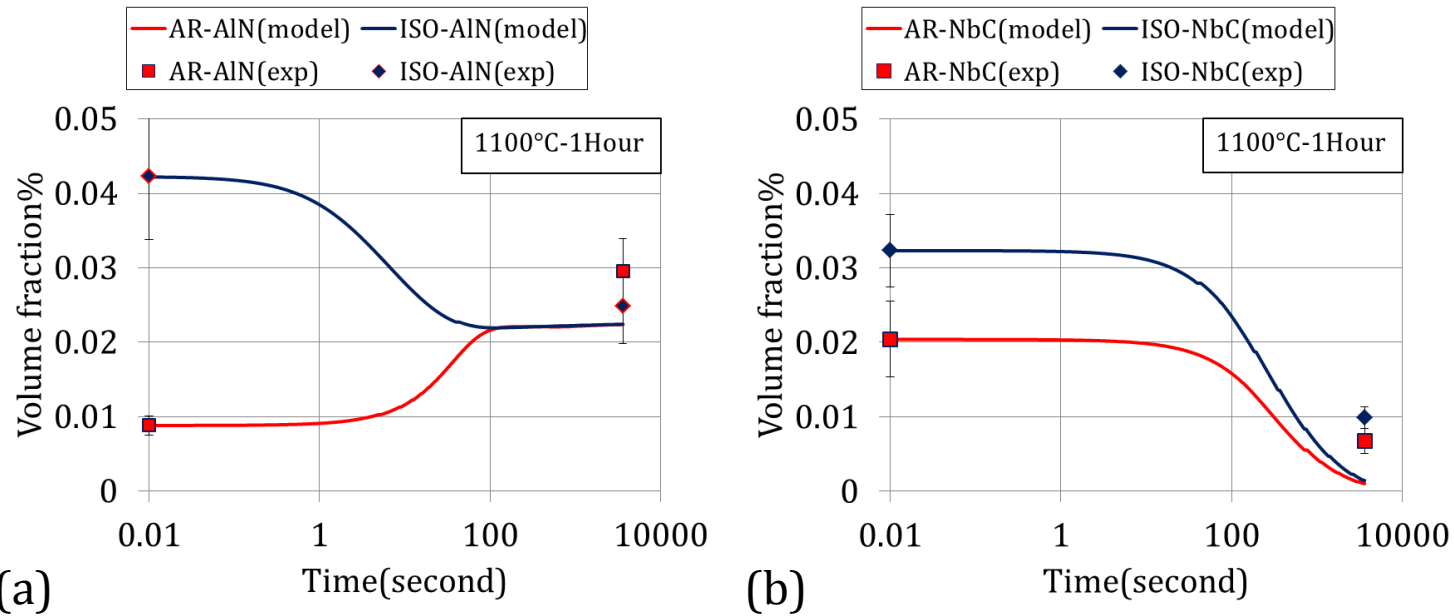

Figure 3.19: Precipitate volume fraction evolution in steel-B's ISO and AR state (a) $\mathrm{AlN}$ at $1100^{\circ} \mathrm{C}$ for $1 \mathrm{hr}$ and (b) $\mathrm{NbC} 1100^{\circ} \mathrm{C}$ for $1 \mathrm{hr}$.

ISO state a decrease in AlN volume fraction can be seen while in the AR state it increases. Both the states attain an equilibrium AlN volume fraction with same quantity approximately at $100 \mathrm{sec}$. NbC precipitate volume fraction evolution is shown for both the AR and ISO state in figure 3.19 (b). At $1100^{\circ} \mathrm{C}$ in both $\mathrm{AR}$ and ISO states NbC volume fraction decreases with time but according to the model prediction, equilibrium is not reached. Although $\mathrm{NbC}$ volume fraction in the AR and ISO states are different at the beginning, after $1 \mathrm{hr}$ of heat treatment at $1100^{\circ} \mathrm{C}$ it attains quite similar quantities. Finally, it can be said from figure 3.19, that model prediction is in agreement with the experimental observation for both $\mathrm{AR}$ and ISO states heat treated at $1100^{\circ} \mathrm{C}$ for $1 \mathrm{hr}$.

\subsubsection{Precipitate mean radius and size distribution evolution}

In this section, the model predictions of precipitate mean radius and size distribution are compared with the experimental measurements. In both steel-A and steel-B, austenite mean grain diameter is $\geq 5 \mu \mathrm{m}$ (see table 3.5 ). It is already shown (see section 3.8.4), when mean grain diameter is larger than $3 \mu \mathrm{m}$, heterogeneous precipitate growth or dissolution has negligible effect on precipitation kinetics. For this reason, it is assumed that the precipitate nucleation and growth or dissolution is occurring homogeneously.

Heat treatments carried out at different temperatures on different states of specimens are modeled taking into account the initial state's precipitate size distribution as input data. In the case of steel-B, heat treatment on both the AR and ISO states done at $900^{\circ} \mathrm{C}$ (just above the $A C_{3}$ ) for 2 min followed by water quench is considered to be initial state. Similarly for steel-A heat treatment at $875^{\circ} \mathrm{C}$ for 

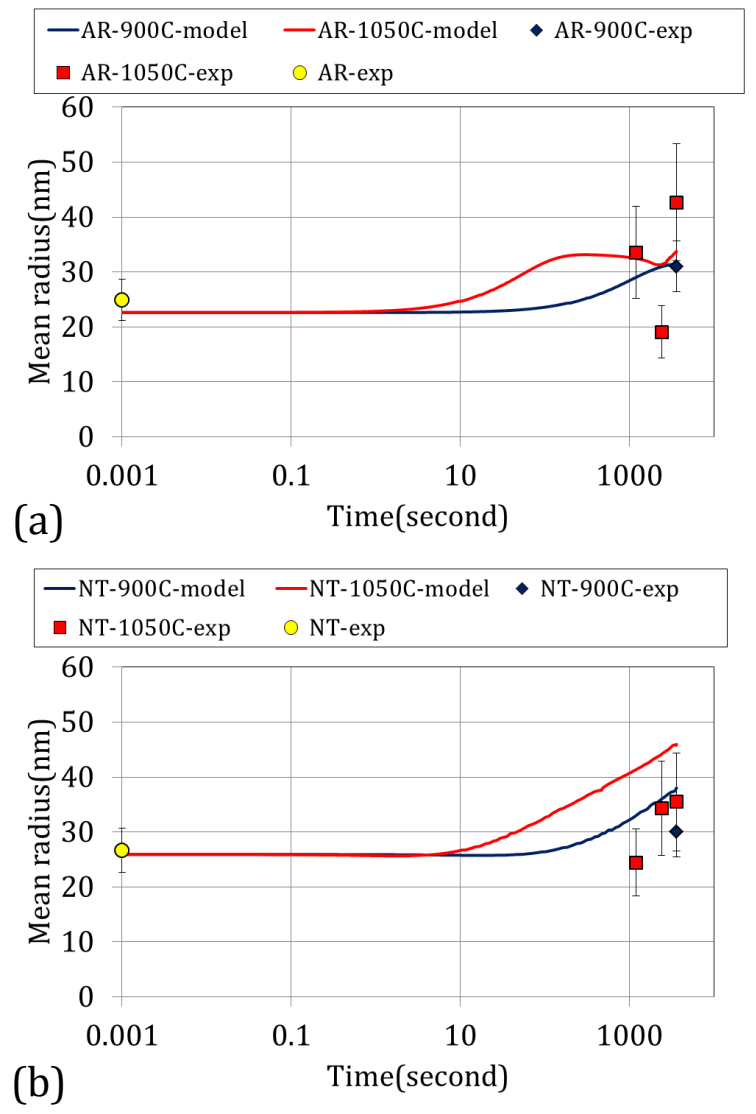

Figure 3.20: Precipitate mean radius evolution in steel-A's heat treated at different temperatures: (a) AR state and (b) NT state.

20 min including the ramping time is considered as the initial state. For all the initial states, an assembly of 1000 classes of precipitate size distribution is taken as input data to model the subsequent heat treatment's effects on evolution.

\subsubsection{Industrial alloy: steel-A}

In figure 3.20, AlN precipitate's mean radius evolution in steel-A is presented in the AR and NT state heat treated at different temperatures. Figure 3.20 (a) represents AlN precipitate's mean radius evolution in the $\mathrm{AR}$ state at $900^{\circ} \mathrm{C}$ and $1050^{\circ} \mathrm{C}$. The model prediction suggests that at $900^{\circ} \mathrm{C}$, AlN precipitate is in the growth stage, as the matrix is not depleted from the solute atoms. On the other hand, at $1050^{\circ} \mathrm{C}$ due to depletion of the solute atoms in the matrix, the precipitate entered the coarsening regime.

A comparison between model predicted precipitate size distribution and experimental measurement in the AR state heat treated at $900^{\circ} \mathrm{C}$ for 1 hour and $1050^{\circ} \mathrm{C}$ for 20, 40 and $60 \mathrm{~min}$ is presented in figure 3.21. Experimental measurement of precipitate size distribution is always limited by the number of representative precipitates measured. Even with a certain degree of error in the measurement, 

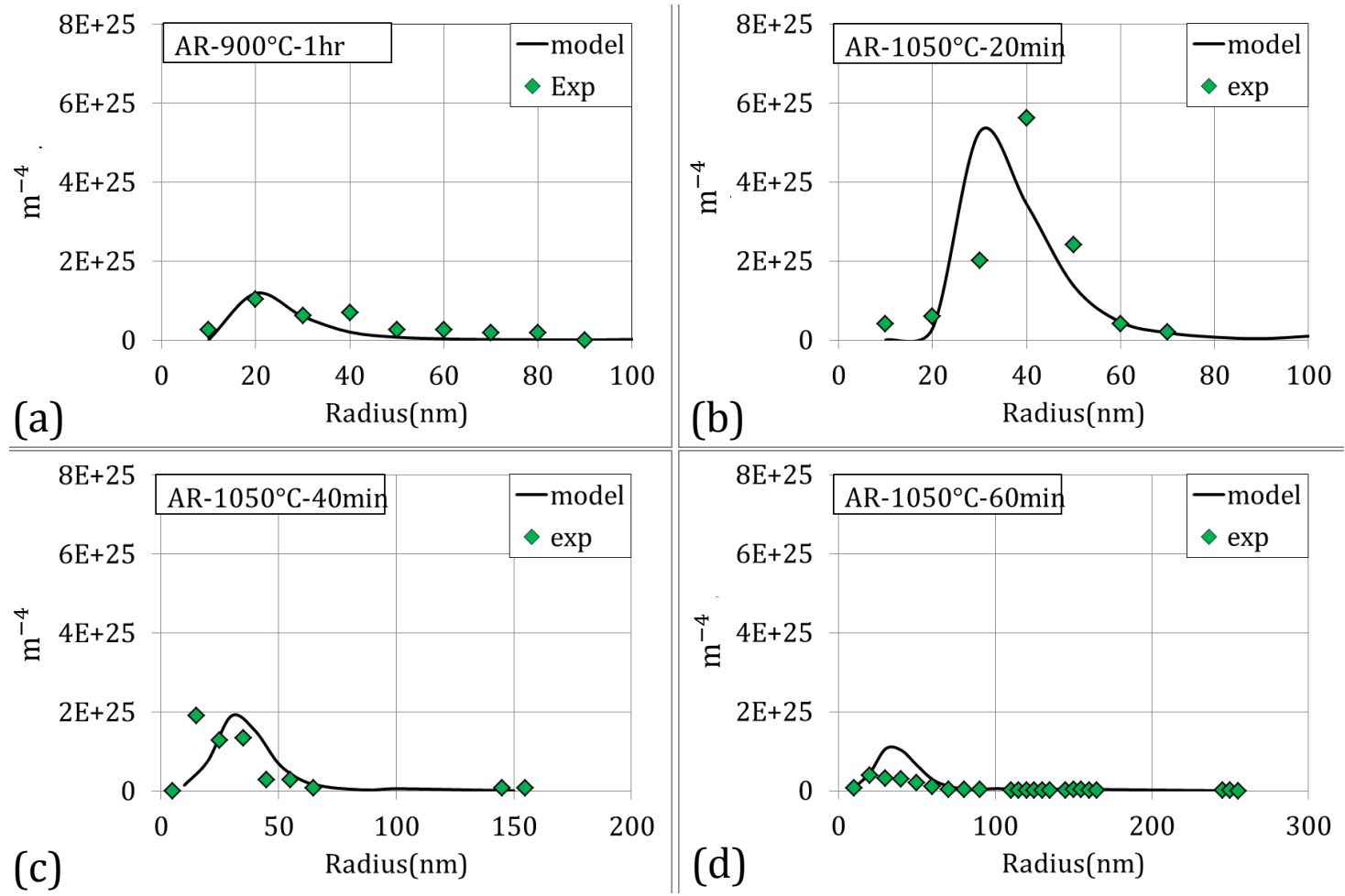

Figure 3.21: Precipitate size distribution evolution in steel-A's AR state heat treated at (a) $900^{\circ} \mathrm{C}$ for $1 \mathrm{hr}$, (b) $1050^{\circ} \mathrm{C}$ for $20 \mathrm{~min}$ (c) $1050^{\circ} \mathrm{C}$ for $40 \mathrm{~min}$ and (d) $1050^{\circ} \mathrm{C}$ for $60 \mathrm{~min}$.

figure 3.21 suggests a reasonable fit between the model prediction and experimental measurement.

Precipitate mean radius evolution in the NT state is presented in figure 3.20 (b). As the NT state itself is a fully precipitate state at the beginning of heat treatment, the more likely growth mechanism during the heat treatment is coarsening. The model prediction has a significant discrepancies with the experimental observation, but the trend in growth is quite similar.

Figure 3.22 is representing a comparison between the model predicted precipitate size distribution and experimental measurement in the NT state heat treated at $900^{\circ} \mathrm{C}$ for $1 \mathrm{hr}$ and $1050^{\circ} \mathrm{C}$ for 20,40 and 60 min. Figure 3.22 suggests a considerable difference between model prediction and experimental measurement of precipitate size distribution in the NT state. The reason can be correlated to the degree of error arises from the statistics of measurement. Besides that precipitate mean radius measurement using carbon extraction replica has its own limitations. It has been observed that larger precipitates are difficult to extract in the replica which contributes to the underestimation of mean radius. 

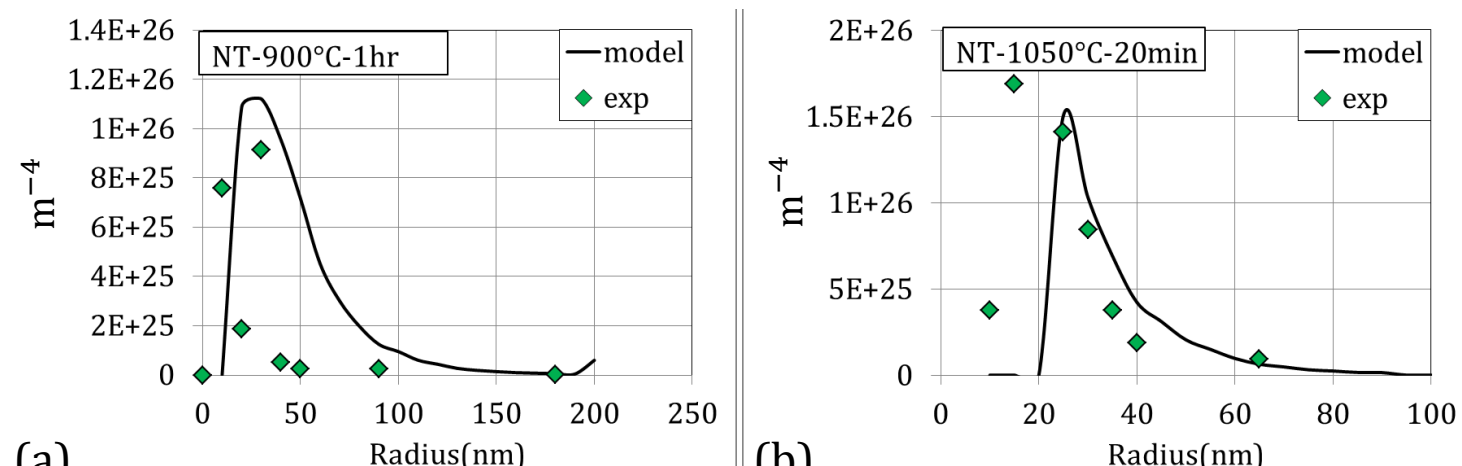

(a)

(b)
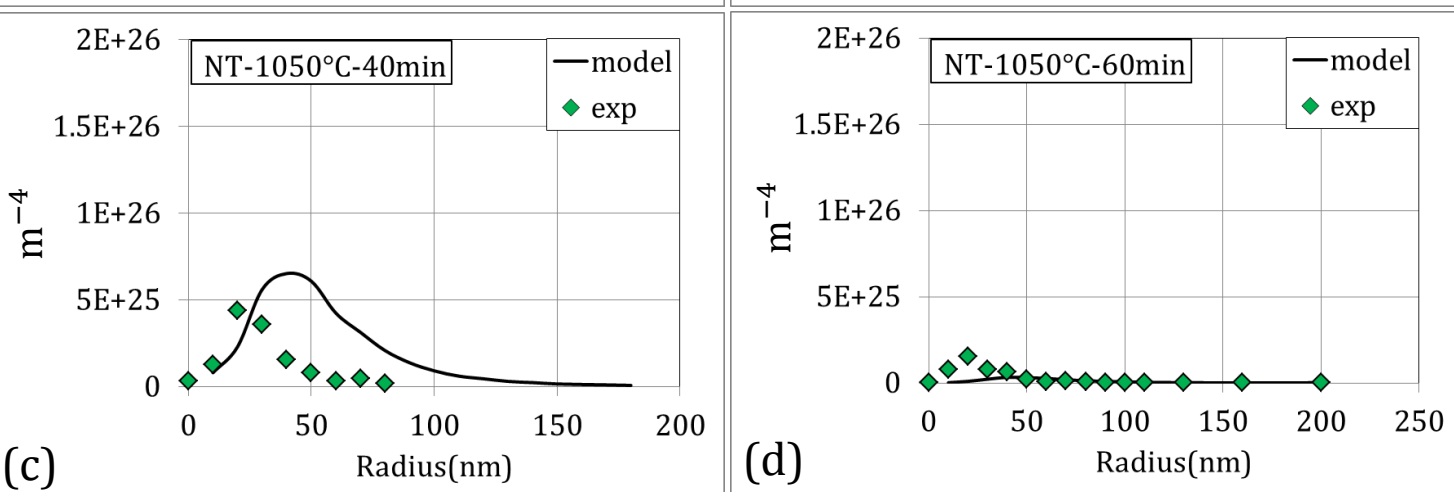

Figure 3.22: Precipitate size distribution evolution in steel-A's NT state heat treated at (a) $900^{\circ} \mathrm{C}$ for $1 \mathrm{hr}$, (b) $1050^{\circ} \mathrm{C}$ for $20 \mathrm{~min}$ (c) $1050^{\circ} \mathrm{C}$ for $40 \mathrm{~min}$ and (d) $1050^{\circ} \mathrm{C}$ for $60 \mathrm{~min}$.
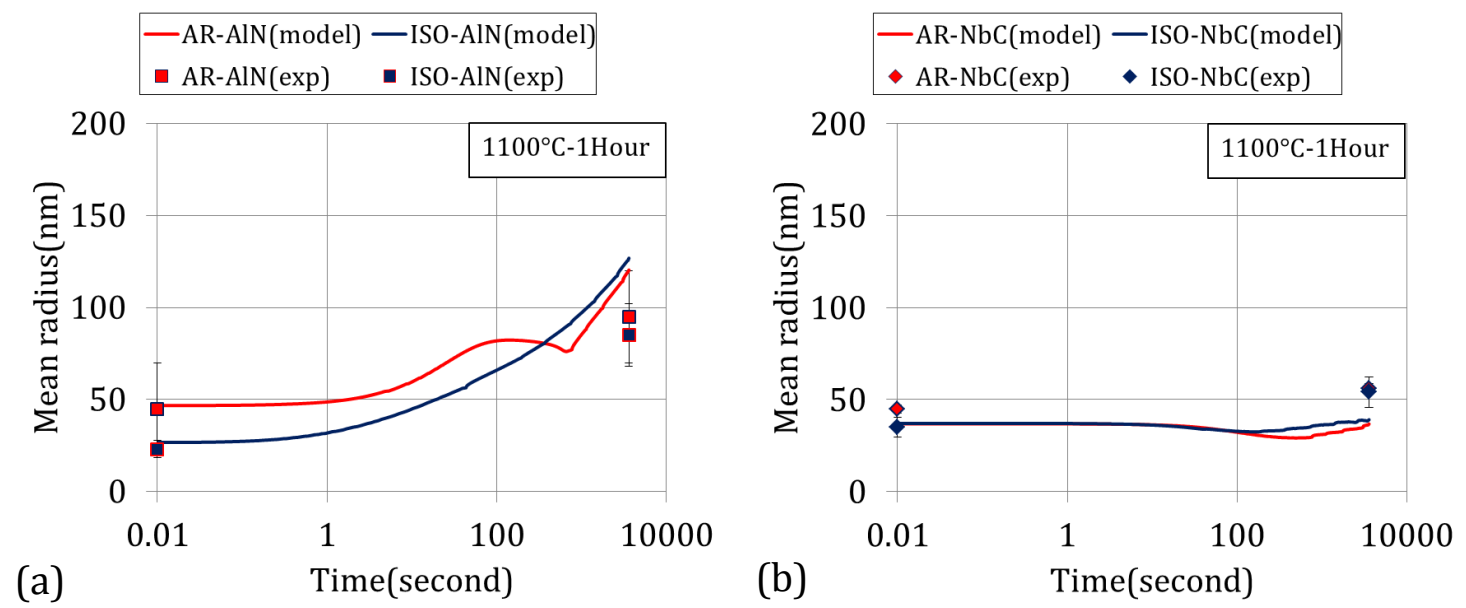

Figure 3.23: Precipitate mean radius evolution in steel-B's AR and ISO state at $1100^{\circ} \mathrm{C}$. (a) AlN mean radius evolution and (b) NbC mean radius evolution. 


\subsubsection{Industrial alloy: steel-B}

In figure 3.23, AlN and NbC precipitate's mean radius evolution in steel-B is presented for $1100^{\circ} \mathrm{C}$ heat treatment. Figure 3.23(a) is representing the AlN mean radius evolution in both the AR and ISO state. The model results suggest that in the AR state AlN grows until $\sim 76$ sec and then coarsening take place. The ISO state shows a linear growth in AlN precipitate. Although the AR and ISO state show quite similar mean radius of AlN precipitate, the growth mechanism is quite different according to the model prediction. Figure 3.23(b) presenting the NbC precipitate evolution in the $\mathrm{AR}$ and ISO state. NbC precipitate mean radius in both the states remains quite unchanged throughout the heat treatment time.

\subsection{Conclusions from this chapter}

In this chapter, a precipitation model is presented with applicability to any industrial alloys. The model is effective in predicting precipitation state in alloy with simple assumptions. The required parameters for modeling are either available is in the literature or experimentally measurable. The experimentally measurable model parameters category includes: (I) precipitate volume fraction by ICP analysis, (II) precipitate chemistry by EDX and III mean radius by HAADF mode in the TEM.

The main conclusion from this chapter can be listed as below:

- Homogeneous and heterogenous precipitate growth is investigated with assumption that precipitate lying in the corner point and grain boundary experience accelerated or heterogenous growth or dissolution.

- Significant change in precipitate growth kinetics is observed when the austenite mean grain size $D_{n}$ is smaller than $3 \mu \mathrm{m}$. In a system with larger than $3 \mu \mathrm{m}$ austenite mean grain size, the heterogeneous growth effects on precipitation kinetics is negligible.

- Precipitation model presented in this chapter is applied to two industrial alloys: steel-A and steel-B. It is observed that the model prediction is quite in agreement with the experimental measurements considering experimental errors. The experimental errors are either arise from statistics of measurement or the technique itself. 
Cette thèse est accessible à l'adresse : http://theses.insa-lyon.fr/publication/2013ISAL0094/these.pdf () [M.A. Razzak], [2013], INSA de Lyon, tous droits réservés 


\section{Chapter 4}

\section{Soft coupled modeling of precipitation and grain growth}

In this chapter, influences of precipitation state evolution on the abnormal and normal grain growth condition is presented by coupling the precipitation (see chapter 3) and Abnormal Grain Growth (AGG) (see chapter 2) model in a soft manner. Here, the term 'Soft Coupled Model (SCM)' is used to emphasize that the precipitation model and the AGG model are executed separately while keeping the time scale similar on both models. So, the precipitation model calculation is completed and the results are then incorporated in the AGG model. Besides the separate execution of the two models, the mean austenite grain size is assumed to be constant throughout the calculation. Finally, prediction of normal and abnormal grain growth from the AGG model is compared with experimental observations.

Considering the complexity of full physical phenomenon, it is not possible to incorporate all the physical properties in the current scheme. A more simplified approach is thus chosen. Selection of physical parameters are incorporated in the current study. The objective is to depict a simplified picture of the precipitation state evolution as a function of time and temperature in relation with abnormal grain growth.

There are previous works regarding coupled modeling of precipitation and grain growth $[5,44,42,69]$ but very few of them dealt with the AGG prediction. Among the available works, Andersen et. al. [5] proposed a coupled modeling approach for the abnormal grain growth and precipitate evolution. In their approach, the precipitate stability is modeled using the solubility product and concurrently the precipitate growth or coarsening is calculated using two different expressions. The biggest limitation of this approach is that the precipitate nucleation is not considered, although it can change both the volume fraction and mean radius and consequently the pinning. 
Militzer et al [44] dealt with the effects of pinning on prior austenite grain growth condition by fitting the experimental grain size data to an empirical power law. They introduced a statistical grain growth model based on Abbruzzese and Luc̈ke [1] proposition. The precipitate induced pinning is expressed as a parameter (pinning parameter) which vary linearly with temperature. Their proposed method is applicable to known alloy where substantial experimental data is available. A fitted pinning parameter constricts the understanding of different aspects effecting the pinning conditions evolution and also grain growth condition itself.

San Martín et al [42] also proposed a coupled model of precipitation and grain growth, quite similar to Andersen et. al. [5]. In their work, prior austenite grain size is modeled considering Zener pinning in the case of normal grain growth. Like Andersen et. al. [5], this work is also limited by only incorporating precipitate mean radius evolution. Precipitate nucleation is not proposed in their coupled approach to model the effects of nucleating precipitate on grain growth. Besides that additional model parameters are required to model the non-isothermal heating rate effects on grain grain growth.

$\mathrm{Xu}$ K. et al [69] presented a model concerning austenite grain size evolution during continuous casting. In their approach, a CNGTs based multi class precipitation model is implemented to predict the precipitation state evolution. In the mean time, precipitation state prediction is utilized in a grain growth model to measure the pinning condition and mean grain size evolution. In the precipitate model the precipitate coarsening is calculated using the general population balance for particle collision (introduced by Smoluchowski [63]). The present modeling approach calculates coarsening by the relative stability of a particular radii class (respect to $R_{k_{b} T}^{*}$ ).

It should be mentioned that apart from the above discussed literatures, there are also other works about grain growth and AGG. In the present limited frame work, the literatures that the author found most relevant with the present work are discussed.

In the current approach of multi class precipitation model (see chapter 3), both the growth and coarsening is handled by consistent expressions applicable to any alloy system. The stability of a particular precipitate class is dependent on the critical precipitate radius $\left(R_{k_{b} T}^{*}\right)$, which depends on the thermodynamic stability. Most of the previous works have modeling parameters requiring optimization in order to produce a reasonable grain growth condition prediction for a particular alloy. In comparison, the current proposed soft coupling approach does not require parameter optimization to produce a consistent picture of the precipitation state evolution and the grain growth condition prediction. 


\subsection{Model assumptions}

In the soft coupled approach, two models: (1) precipitation model and (2) AGG model are coupled. The individual model assumption of (1) precipitation model is discussed in the chapter 3 and (2) AGG model in chapter 2. The soft coupled modeling is based on the following assumptions:

1. In the soft coupled model, it is assumed that the mean austenitic grain is constant throughout the heat treatment.

2. Precipitate nucleation and growth occur homogeneously.

3. Abnormal grain growth model states that the largest precipitates lie in the corner points. As it is assumed that the grain boundary remains stationary, stable precipitation state allows that precipitates that lie in the corner points remain there throughout the simulation.

4. The grain boundary and corner point precipitate dissolution allow the corner point to move enough (not changing the mean grain size significantly) to get pinned on the subsequent larger precipitate.

\subsection{Modeling strategy}

In the current modeling approach (see figure 4.1), a static coupling of two modeling scheme is chosen. Here, the precipitation modeling module give the measure of precipitation state evolution as a function of time and temperature. Considering the thermodynamic stability of the precipitate in the matrix, the precipitation model calculates: volume fraction $\left(f_{v}(T, t)\right)$, mean radius $(\bar{r}(T, t))$ and size distribution $\left(N_{i} r_{i}(T, t)\right)$ as a function of time and temperature. The precipitation model results are taken as input in the AGG model. The AGG model calculates the evolution of pinning condition as a function of time and temperature taking into account the initial austenite mean grain size $\left(D_{n}^{0}\right)$. The initial austenite mean grain size is referred to the mean grain size occurs just after the martensitic or bainitic microstructure transforms to austenite.

A schematic representation of the simulation setups is shown in figure 4.2. Isothermal heat treatment simulation (see figure 4.2 (a)) is executed starting from the target temperature at time $t_{0}=0$ sec. Here, the holding time is the difference between the end time $t_{1}$ and the starting time $t_{0}$. In the isothermal heat treatment simulation, the effects of heating from room temperature to the target is not considered. It is assumed that in a pre-heated furnace (to the target temperature), the heating rate is fast enough to have insignificant effects on precipitation state and austenite grain growth. Effects of heating rate is simulated (see figure 4.2 (b)) 


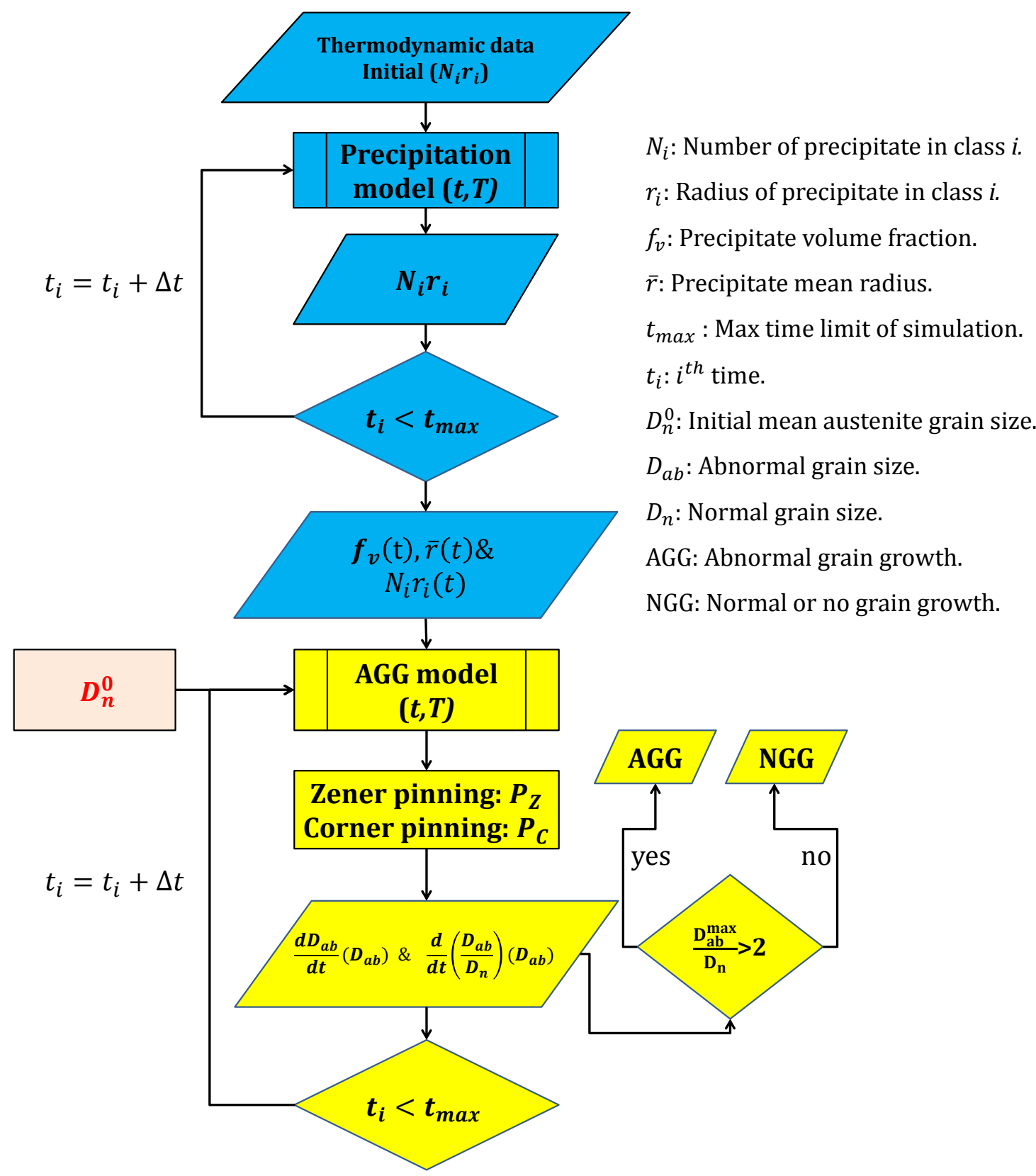

Figure 4.1: Schematic representation of soft coupling between precipitation and abnormal grain growth model. 


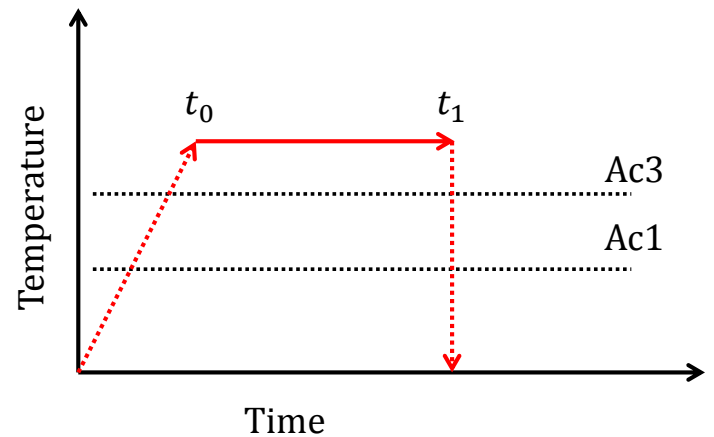

(a) Isothermal heat treatment setup

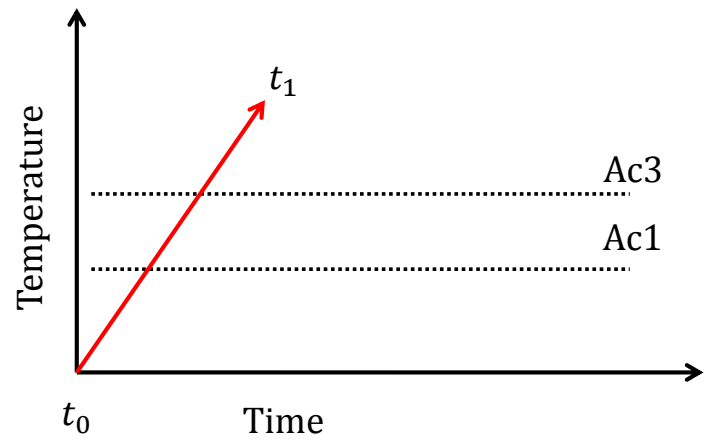

(b) Heating rate simulation setup

Figure 4.2: Schematic representation of simulation setup: (a) isothermal heat treatment setup and (b) heating rate simulation setup.

by assuming at initial time $t_{0}$ the specimen is at room temperature and at the final time $t_{1}$ it reaches target temperature. The total heating time is the difference between final time $t_{1}$ and initial $t_{0}$. In the following section, effects of heating rate is not simulated using the SCM approach. The simulation setup is presented to depict a difference between isothermal and heating rate simulation setup.

In this approach, the mutual interaction between mean grain growth and precipitation state is not taken into account. Moreover the grain growth conditions are modeled assuming that the material has a static mean grain size with evolving precipitation state. So in a sense, only the evolution of Zener and corner pinning conditions are modeled which in fact effects the grain growth conditions (Abnormal Grain Growth (AGG) or Normal/No Grain Growth (NGG)) in the material. Particularly the soft coupled modeling approach is chosen to simplify the complex system where the austenite grain growth takes place along with the precipitation state evolution. The increase in mean austenite grain size changes the total number of corner points in the system, thus changing the total pinning pressure (change in corner pinning pressure but the Zener pinning will remain constant). Besides the change in pinning pressure, an increase in mean grain size also decreases the mean grain growth pressure. In the case of pre-existing larger grains, larger mean grain size effectively decreases the number of possible neighbors thus lowers the AGG driving pressure. Concurrent change in three physical parameters with mean grain size in the system rather offers complexity in isolating the effects of precipitation state on AGG. By assuming static austenite grain size, efforts are given to depict a simplified picture of AGG and NGG in relation with pinning condition evolution. 


\subsubsection{Conditions for abnormal grain growth}

The abnormal grain growth model is presented in details in chapter 2 . In this section, the abnormal grain growth condition is recalled briefly. As presented in chapter 2, abnormal grain growth initiation and propagation is possible if the abnormal grain growth rate is positive and also comparatively larger than the mean grain growth rate in the system. The condition for AGG is given below:

$$
\frac{d}{d t}\left(\frac{D_{a b}}{D_{n}}\right)>0
$$

The abbreviated form of the AGG condition is given below:

$$
\begin{aligned}
\frac{d}{d t}\left(\frac{D_{a b}}{D_{n}}\right) & =\frac{1}{D_{n}} \frac{d D_{a b}}{d t}-\frac{D_{a b}}{D_{n}^{2}} \frac{d D_{n}}{d t} \\
& =\frac{2 \gamma_{g} M}{D_{n}^{2}}\left[\mu-(1-\mu)\left(\frac{D_{n}}{D_{a b}}\right)^{2}+(2 \mu-1) \frac{D_{n}}{D_{a b}}-\frac{\lambda D_{a b}}{2 D_{n}}\right] \\
& -\frac{M}{D_{n}}\left(\frac{D_{a b}}{D_{n}}+1\right)\left(P_{C}+P_{Z}\right)
\end{aligned}
$$

Here, $P_{Z}$ is the Zener pinning and $P_{C}$ is the corner pinning.

$$
M=M_{0} \exp \left(-\frac{Q}{R T}\right)
$$

Here, $D_{a b}$ can vary between $D_{n}$ and $\infty$. Austenite grain boundary mobility $M$ is expressed in equation 4.3 , where $M_{0}=3.16 \times 10^{-2} \mathrm{~m}^{2} / \mathrm{s}$ is the pre-exponential factor, $Q=300 \mathrm{~kJ} / \mathrm{mol}$ is the grain boundary activation energy [45], $R$ is the gas constant and $T$ is the temperature in Kelvin.

The expression of AGG condition 4.2 is in quadratic form. There are two values of $D_{a b}$ delimiting a domain within which the AGG condition is fulfilled. The smallest value of $D_{a b}$ can be denoted as $D_{a b}^{\min }$ and the largest as $D_{a b}^{\max }$ (see figure 4.3). $D_{a b}^{\min }$ and $D_{a b}^{\max }$ is representing the range of grains in a distribution which can grow comparatively faster than the mean size grains. In a zero pinning condition (no precipitate), for $\mu=0.56$ and $\lambda=0.6$, it can be found that AGG condition is valid between, $D_{a b}^{\min } / D_{n} \approx 1.26$ and $D_{a b}^{\max } / D_{n} \approx 1.43$.

In the figure $4.3, d / d t\left(D_{a b} / D_{n}\right)$ obtained for increasing pinning conditions are superimposed on a normal grain size distribution. As shown in figure $4.3, D_{a b}^{\max }$ can be within the grain size distribution and very close to the mean sized grain (1) or close but smaller than the largest grain size (2) or larger than the maximum grain size in the distribution (3). Among these three situations only (3) bearing the 


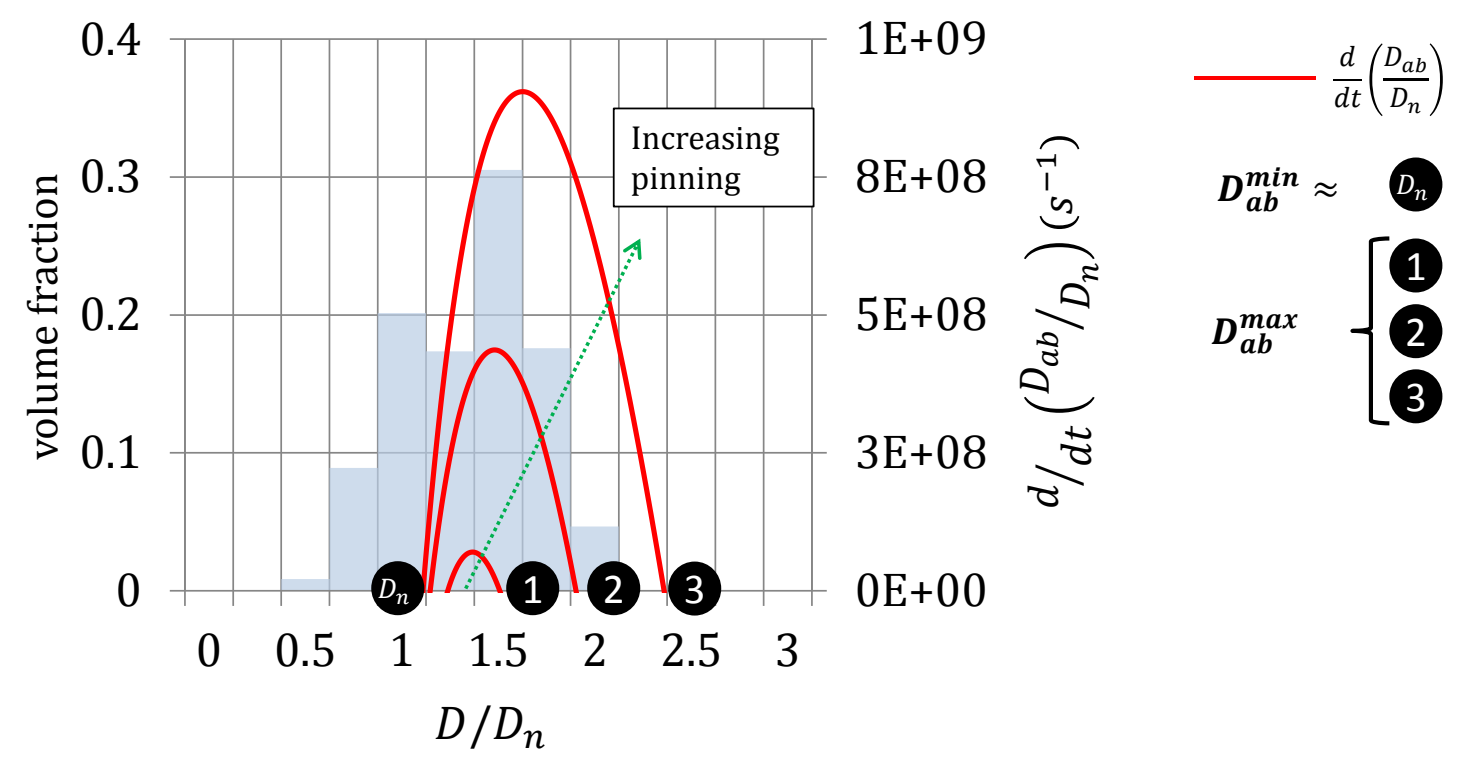

Figure 4.3: Superimposition AGG condition $\left(d / d t\left(D_{a b} / D_{n}\right)>0\right)$ on grain size distribution. $d / d t\left(D_{a b} / D_{n}\right)$ can range between $D_{n}$ and (1) or (2) or (3). Here, it is assumed that $M=1$.

probability of AGG initiation. In (3), the largest grains in the microstructure can grow relatively faster than the mean. In the case of, (1) and (2) grain sizes close to the mean sized one grow faster which facilitate a quasi-stationary grain size distribution evolution (normal grain growth). From figure 4.3, it can be concluded that $D_{a b}^{\max }$ must be at least 2 times of $D_{n}$ in order for AGG to occur. So, a realistic qualitative identifier of AGG and NGG can be expressed as follows:

$$
\begin{array}{ll}
D_{a b}^{\max } / D_{n}>2 \text { Probable grain growth condition: AGG } \\
D_{a b}^{\max } / D_{n}<2 \text { Probable grain growth condition: NGG }
\end{array}
$$

In a static system with stationary mean grain $\left(D_{n}\right)$ size and pinning condition, it is sufficient to present $d / d t\left(D_{a b} / D_{n}\right)$ as a prediction of AGG. On the other hand, system with evolving pinning conditions, $D_{a b}^{\max } / D_{n}$ provides a much simpler representation. In order to present the evolution of AGG condition with time, $D_{a b}^{\max } / D_{n}$ is presented as the parameter showing AGG probability in the studied system.

\subsection{Implications of soft coupled model}

The abnormal grain growth and the precipitation model are presented in detail in chapter 2 and chapter 3. In order to study different aspects of the soft coupled approach, it is quite important to emphasize on parameters controlling grain growth condition separately. From the point of view of possible precipitation states in low 

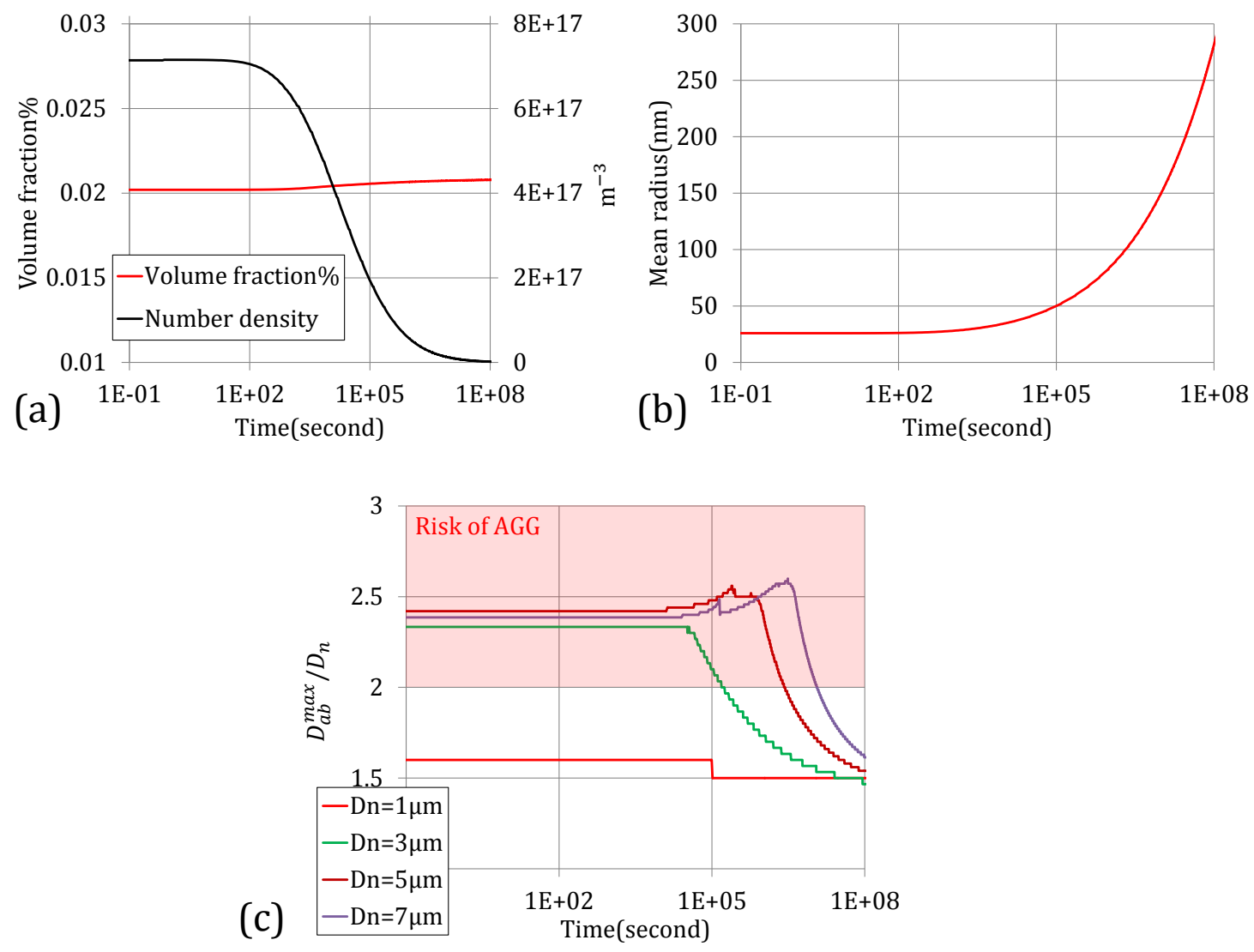

Figure 4.4: (a) Volume fraction and number density evolution of a precipitate, (b) precipitate mean radius evolution and (c) maximum grain size $\left(D_{a b}^{\max }\right)$ relative to the mean grain size $\left(D_{n}\right)$ which has the probability to grow abnormally. Here, temperature $T=900^{\circ} \mathrm{C}$, the model parameters $\mu=0.56$ and $\lambda=0.6$.

alloy steel, the criteria of study can be divided into three major classes:

1. Grain growth condition in presence of precipitate coarsening.

2. Grain growth condition in presence of precipitate nucleation.

3. Grain growth condition in presence of dissolving precipitate.

These three precipitate evolution conditions listed, cover almost all the precipitate evolution conditions that can be found in the industrial alloy during austenitizing treatment. In the following discussion, all the precipitation state is modeled assuming homogeneous growth and dissolution.

\subsubsection{Abnormal grain growth in presence of precipitate coars- ening}

In figure 4.4 (a) and (b), the evolution of a coarsening precipitation state are shown. Here, the precipitate volume fraction (see figure 4.4(a)) remains constant 
but the number density decreases due to coarsening. Precipitate coarsening can also be observed by the increase in mean radius (see figure 4.4(b)).

Figure 4.4(c) represents the ratio between the maximum grain size $\left(D_{a b}^{\max }\right)$ with the probability to grow abnormally $\left(d / d t\left(D_{a b} / D_{n}\right)>0\right)$ and the mean grain size $\left(D_{n}\right)$. In this precipitate coarsening condition, the results suggest that there is no possibility of abnormal grain growth for the mean grain size of $1 \mu \mathrm{m}\left(D_{a b}^{\max } / D_{n}<2\right)$. $1 \mu \mathrm{m}$ mean grain size has higher growth pressure $\left(d D_{n} / d t\right)$ than 3,5 and $7 \mu \mathrm{m}$. The pre-existing larger grains (at least 2 times larger than $D_{n}$ ) in the microstructure can not growth comparatively faster thus no probability of AGG.

On the other hand, figure 4.4(c) shows increase in $D_{a b}^{\max } / D_{n}$ with the increase in initial $D_{n}$. Besides that at larger precipitate mean radius ( $>60 \mathrm{~nm}$ ) there is a decrease in the maximum grain size that lies in the risk of AGG. It is understandable that a decrease in precipitate number density decreases the total pinning force. A decrease in pinning effectively increases the mean grain growth rate and reduces the risk of AGG. It is also noticeable from figure 4.4 that larger mean grain sizes take more time to shift from AGG to NGG condition. From kinetics point of view, this observation refers to the equilibrium persists between the mean grain growth rate and available pinning in the microstructure. It can be concluded that in a preexisting AGG condition, AGG probability decreases with precipitate coarsening (decreasing number density).

\subsubsection{Abnormal grain growth in presence of precipitate nu- cleation}

In figure 4.5 (a), evolution of nucleating precipitate volume fraction and number density are shown. Here, the precipitate volume fraction and number density starting from zero increases with time. Simultaneously, precipitate growth occurs which can be seen by the increase in mean radius (see figure $4.5(\mathrm{~b})$ ).

Figure 4.5 (c) represents the ratio between the maximum grain size $\left(D_{a b}^{\max }\right)$ with probability to grow abnormally $\left(d / d t\left(D_{a b} / D_{n}\right)>0\right)$ and the mean grain size $\left(D_{n}\right)$. It can be seen from figure 4.5 (c) that there is no probability of AGG for all the mean grain sizes as $D_{a b}^{\max } / D_{n}<2$ before $10000 \mathrm{sec}$. As the grain size with the probability to grow comparatively faster is very close to the mean grain size $\left(D_{n}\right)$, the system should remain quasi-stationary (NGG). Above $10000 \sec D_{a b}^{\max } / D_{n}>2$, the larger grains in the system start to grow faster than the mean sized grains $\left(D_{n}\right)$ and can experience AGG.

It is noticeable that $1 \mu \mathrm{m}$ mean grain size never enters the AGG risk region. $1 \mu \mathrm{m}$ grain size has higher growth pressure and the larger grains $\left(>1.5 D_{n}\right)$ can not grow comparatively faster. As a result, AGG is not probable. If we compare 

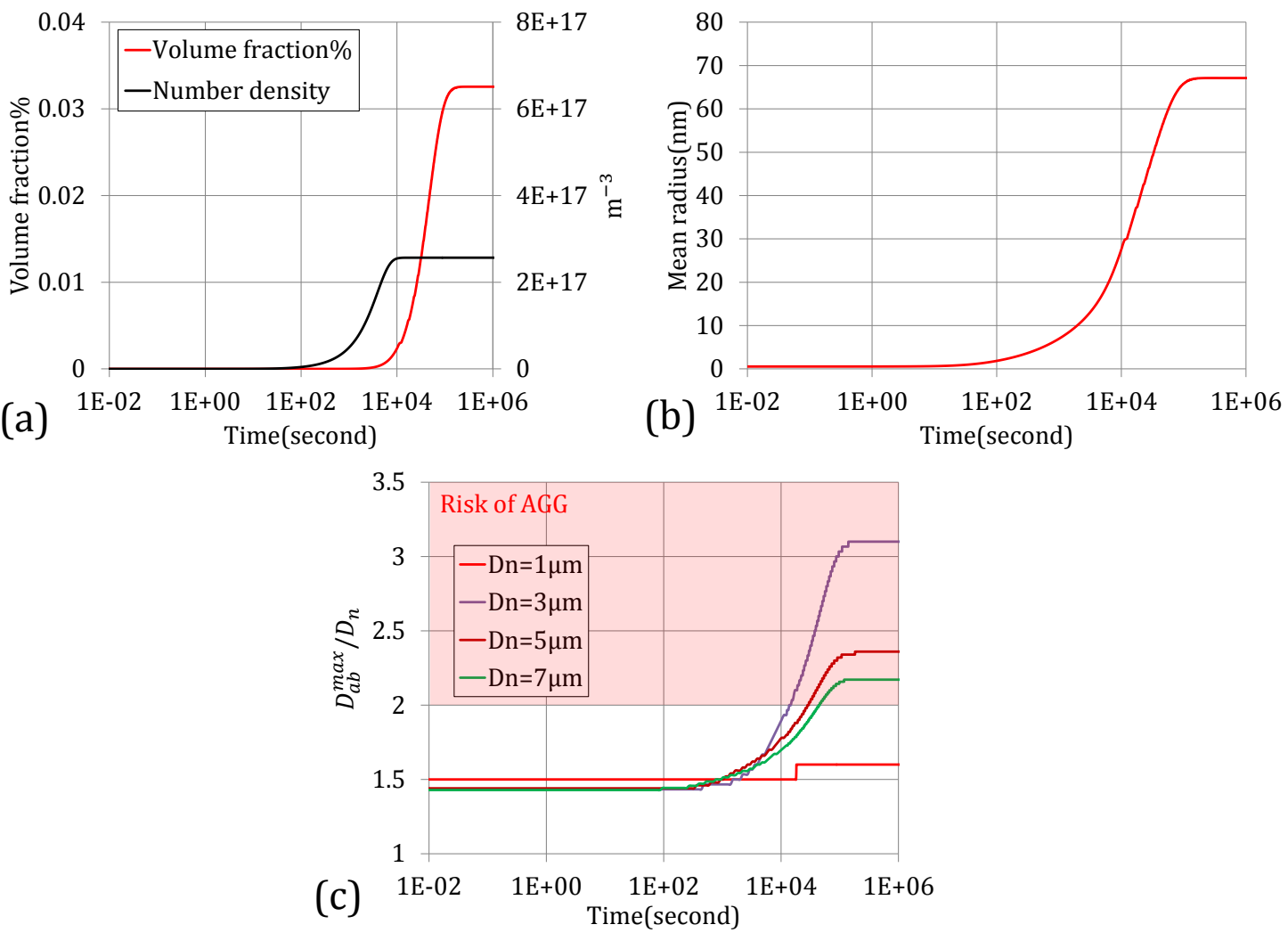

Figure 4.5: (a) Volume fraction and number density evolution of a nucleating precipitate, (b) precipitate mean radius evolution and (c) maximum grain size $\left(D_{a b}^{\max }\right)$ relative to the mean grain size $\left(D_{n}\right)$ which has the probability to grow abnormally. Here, temperature $T=800^{\circ} \mathrm{C}$, the model parameters $\mu=0.56$ and $\lambda=0.6$. 


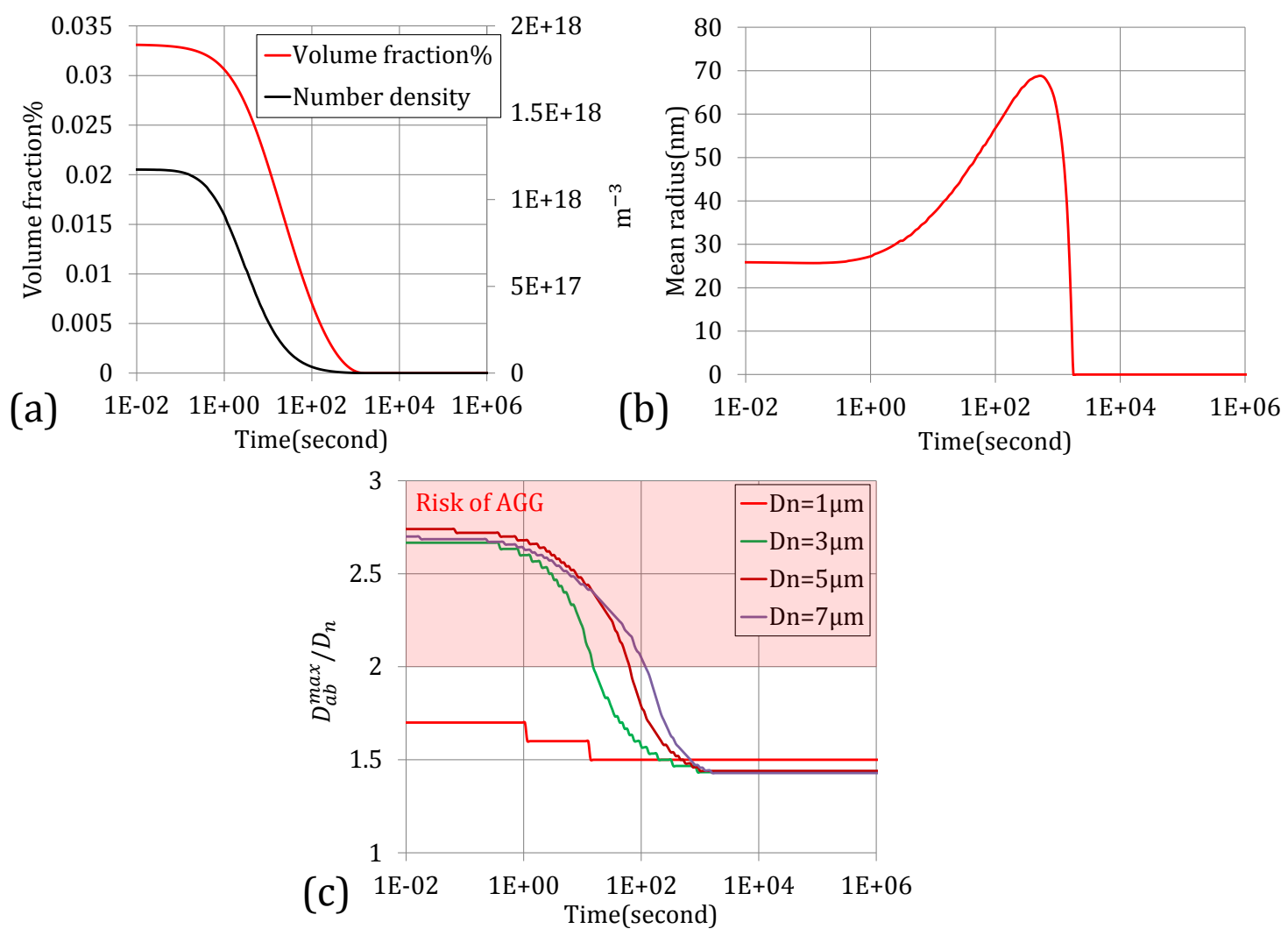

Figure 4.6: (a) Volume fraction and number density evolution of a dissolving precipitate, (b) precipitate mean radius evolution and (c) maximum grain size $\left(D_{a b}^{\max }\right)$ relative to the mean grain size $\left(D_{n}\right)$ which has the probability to grow abnormally. Here, temperature $T=1100^{\circ} \mathrm{C}$, model parameter $\mu=0.56$ and $\lambda=0.6$.

between mean grain size of 3 to $7 \mu \mathrm{m}$, smaller grain size enters the AGG risk zone earlier. In this mean grain size range, the larger $\left(D_{a b}\right)$ grains can overcome the pinning and also grow comparatively faster $\left(d / d t\left(D_{a b} / D_{n}\right)>0\right)$. The smaller mean grain size offers higher quantity of grain boundary surface area around the preexisting larger grains thus the driving pressure $d D_{a b} / d t$ is higher. Higher $d D_{a b} / d t$ consequently drive $D_{n}=3 \mu \mathrm{m}$ in to the AGG risk zone earlier which is followed by 5 and $7 \mu \mathrm{m}$.

Finally it can be concluded from figure 4.5 (a), (b) and (c), that abnormal grain growth probability increases in a system when there is a certain volume fraction and number density of precipitates. During precipitate nucleation microstructure with smaller mean grain size $(3 \mu m)$ experience AGG earlier than comparatively larger ones. 


\subsubsection{Abnormal grain growth in presence of dissolving pre- cipitate}

In figure 4.6 (a), the precipitate volume fraction and number density evolutions of a dissolving precipitate are shown. Here the precipitate volume fraction and number density (see figure 4.6(a)) decreases with time and finally completely complete dissolution occurs. Initially an increase in the precipitate mean radius can be seen (see figure 4.6(b)) as the smaller precipitate classes in the distribution dissolve faster than the large ones. When there are only the larger precipitate classes remain, the mean radius decreases rather quickly.

In figure $4.6(\mathrm{c})$, the ratio between maximum grain size $\left(D_{a b}^{\max }\right)$ with probability to grow abnormally $\left(d / d t\left(D_{a b} / D_{n}\right)>0\right)$ and the mean grain size $\left(D_{n}\right)$ is presented. It can be seen from figure 4.6 (c) that for all the investigated mean grain size $\left(D_{n}\right)$ except $1 \mu \mathrm{m}$, initially the system has a probability to undergo AGG $\left(D_{a b}^{\max } / D_{n}>2\right)$. $D_{a b}^{\max } / D_{n}$ shows a gradual decrease with decreasing precipitate density and finally reaches $D_{a b}^{\max } / D_{n} \approx 1.5$ above 10000 second. The probability of AGG occurrence indeed diminishes just above 100 second into the simulation as $D_{a b}^{\max } / D_{n}<2$. From the above discussion it can be stated that the possibility of AGG occurrence decreases with the decreasing precipitate volume fraction and number density. In absence of precipitate there is no probability of AGG in the system.

\subsection{Application to low alloy steel}

The soft coupled model application in two industrial alloys are discussed in this section. The SCM predictions are validated with experimental results. The modeling results agreements and disagreements are discussed in order to clarify the capabilities and limitations of the soft coupled approach.

In the following discussion, two studied alloys: (1) steel-A and (2) steel-B are presented in details. The compositions and heat treatment cycles are also discussed. Experimentally measured precipitation states and the austenite grain size distributions are listed for the different states of the alloys. A comparison between the different states of alloys are done to understand the relation between precipitation state and AGG.

\subsubsection{Industrial alloy: steel-A}

\subsubsection{Composition and heat treatment}

The composition of the industrial alloy steel-A is given in table 4.1. The main precipitating element in this alloy is Al. The Energy Dispersed Spectroscopy (EDS) 
Table 4.1: Composition of the industrial alloy steel-A in atomic \%

\begin{tabular}{cccccccccc}
\hline $\mathrm{C}$ & $\mathrm{Si}$ & $\mathrm{Mn}$ & $\mathrm{Ni}$ & $\mathrm{Cr}$ & $\mathrm{V}$ & $\mathrm{Ti}$ & $\mathrm{Al}$ & $\mathrm{Nb}$ & $\mathrm{N}$ \\
0.702 & 0.407 & 0.455 & 1.22 & 1.47 & 0.00696 & 0.00194 & 0.0549 & 0.00171 & 0.0598 \\
\hline
\end{tabular}

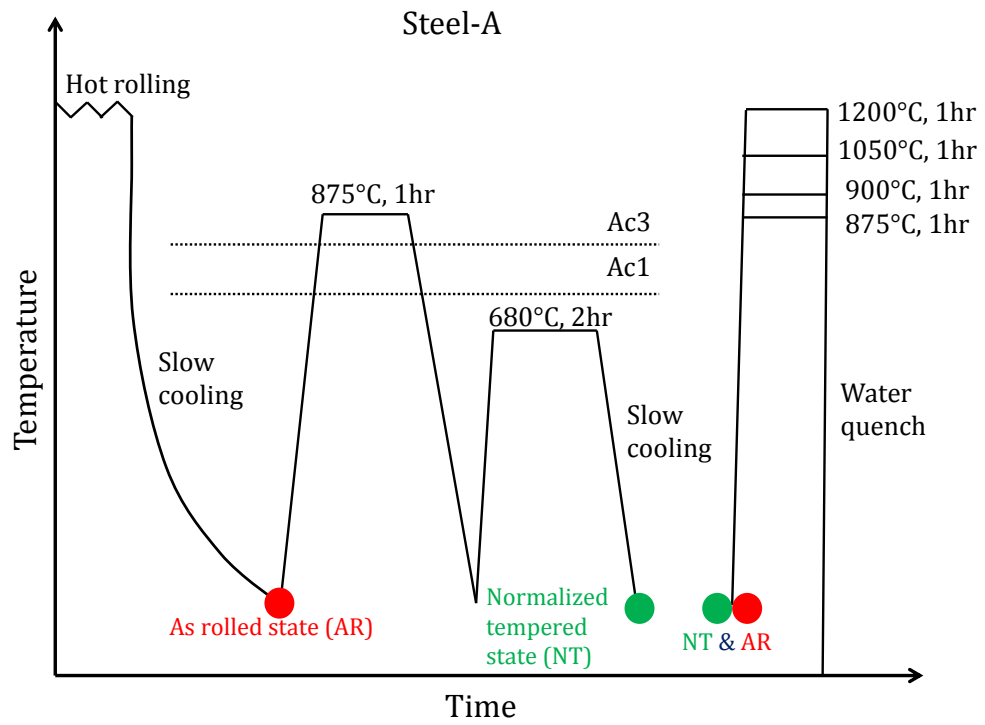

Figure 4.7: Heat treatment cycle of industrial alloy steel-A to obtain As Rolled (AR) and Normalized Tempered (NT) state.

analysis of the precipitate on carbon extraction replica in a Transmission Electron Microscope (TEM) confirmed that the precipitate's chemistry is AlN. Besides that Induction Coupled Plasma (ICP) analysis of the precipitate also showed that the AlN quantity is only large enough to impact on grain growth (experimental procedure given in appendix A).

Heat treatments done on the steel-A are shown in figure 4.7. The As Rolled (AR) state is obtained by hot rolling of the as cast section. The AR state is then heat treated above $\left(>A C_{3}\right)$ at $875^{\circ} \mathrm{C}$ for $1 \mathrm{hr}$ and $\left(<A C_{1}\right)$ at $680^{\circ} \mathrm{C}$ for $2 \mathrm{hr}$ followed by air cooling to obtain the Normalized Tempered (NT) state. Metallographic analysis suggested that both AR and NT state's microstructure is bainitic.

Austenitizing treatment is performed on both AR and NT states at $875^{\circ} \mathrm{C}$, $900^{\circ} \mathrm{C}, 1050^{\circ} \mathrm{C}$ and $1200^{\circ} \mathrm{C}$ for different holding time. The samples are quenched in order to retain austenite grain boundaries impression in the microstructure. The quenched sample's prior austenite grain boundaries are revealed by the hot picric acid solution modified with a surface reactant (see appendix A for details). The precipitate volume fraction in a heat treated state is measured by dissolving the matrix using a potentiostat in a solution of salicylic acid, lithium chloride and methanol. The filtered precipitates dissolved in a highly acidic solution $(H C L+$ 
$\left.\mathrm{H}_{2} \mathrm{SO}_{4}+\mathrm{HF}+\mathrm{HNO}_{3}\right)$ are then analyzed in the ICP to measure the precipitate quantity (see appendix A for details). Precipitate volume fraction is measured in terms of substitutional element mass fraction in PPM. The PPM can be converted to the precipitate volume fraction using the expression 4.5 .

$$
f_{v}^{A_{x} B_{y}}(T, t)=f_{m}^{A} \frac{\rho_{F e}}{\rho_{A_{x} B_{y}}}\left(\frac{y}{x} \frac{M_{B}}{M_{A}}+1\right)
$$

Here, $f_{v}^{A_{x} B_{y}}(T, t)$ is the volume fraction of $A_{x} B_{y}$ precipitate, $f_{m}^{A}$ is the mass fraction of element $A$ measured using ICP, $\rho_{F e}$ and $\rho_{A_{x} B_{y}}$ are the molecular density of the matrix and the precipitate and $M_{A}$ and $M_{B}$ are the molar mass of the substitutional and interstitial elements.

Precipitate size distribution is measure by analyzing carbon extraction replica in TEM using the High Angle Annular Dark Field (HAADF) mode. Images taken using the HAADF mode are analyzed by image processing software to obtain the equivalent circle radius of the precipitates. The measured precipitate radiuses are used to build the size distribution (see appendix A). Normalizing the size distribution with the volume fraction give us the number density (Number of precipitate $/ \mathrm{m}^{-3}$ ) of the precipitate classes. In the present study, precipitate size distribution is presented as $\mathrm{m}^{-4}$ where the number density per class $\left(\mathrm{m}^{-3}\right)$ is divided by the class width $(\Delta R)$.

\subsubsection{Precipitation state and grain size evolution}

In figure 4.8 and 4.9 , the AR state's precipitate size distribution with corresponding micrograph is presented. Figure $4.8\left(A_{2}\right)$ and $\left(B_{2}\right)$ represent the precipitate size distribution in $\mathrm{AR}$ and $\mathrm{AR}$ state heat treated at $900^{\circ} \mathrm{C}$ for $1 \mathrm{hr}$. Similarly figure 4.9, shows the precipitate size distributions of AR state heat treated at $1050^{\circ} \mathrm{C}$ for 20,40 and $60 \mathrm{~min}$. It can be observed from figure 4.9 that with increasing heat treatment time at $1050^{\circ} \mathrm{C}$, the size distribution becomes wider. Moreover in the $1050^{\circ} \mathrm{C}-60$ min heat treated state, a bimodal size distribution is obtained.

Prior austenite grain size distribution of the AR state heat treated at different temperatures are shown in figure 4.10 and 4.11. From figure 4.10, it can be seen that the prior austenite grain size distribution remained quasi-stationary (normal growth) for the heat treatment at $900^{\circ} \mathrm{C}$ and $1200^{\circ} \mathrm{C}$ for $1 \mathrm{hr}$. At $1200^{\circ} \mathrm{C}$ the grain sizes are comparatively larger as all the precipitates are dissolved in the matrix and the austenite grains could grow without any hinderance. In figure 4.11, prior austenite grain size distribution of the AR state heat treated at $1050^{\circ} \mathrm{C}$ for 20,40 and $60 \mathrm{~min}$ is presented. All the grain size distributions along with the adjacent micrographs show that the grain growth mode at this stage is Normal Grain Growth (NGG). From figure 4.10 and 4.11, it can be said that for all the presented heat 


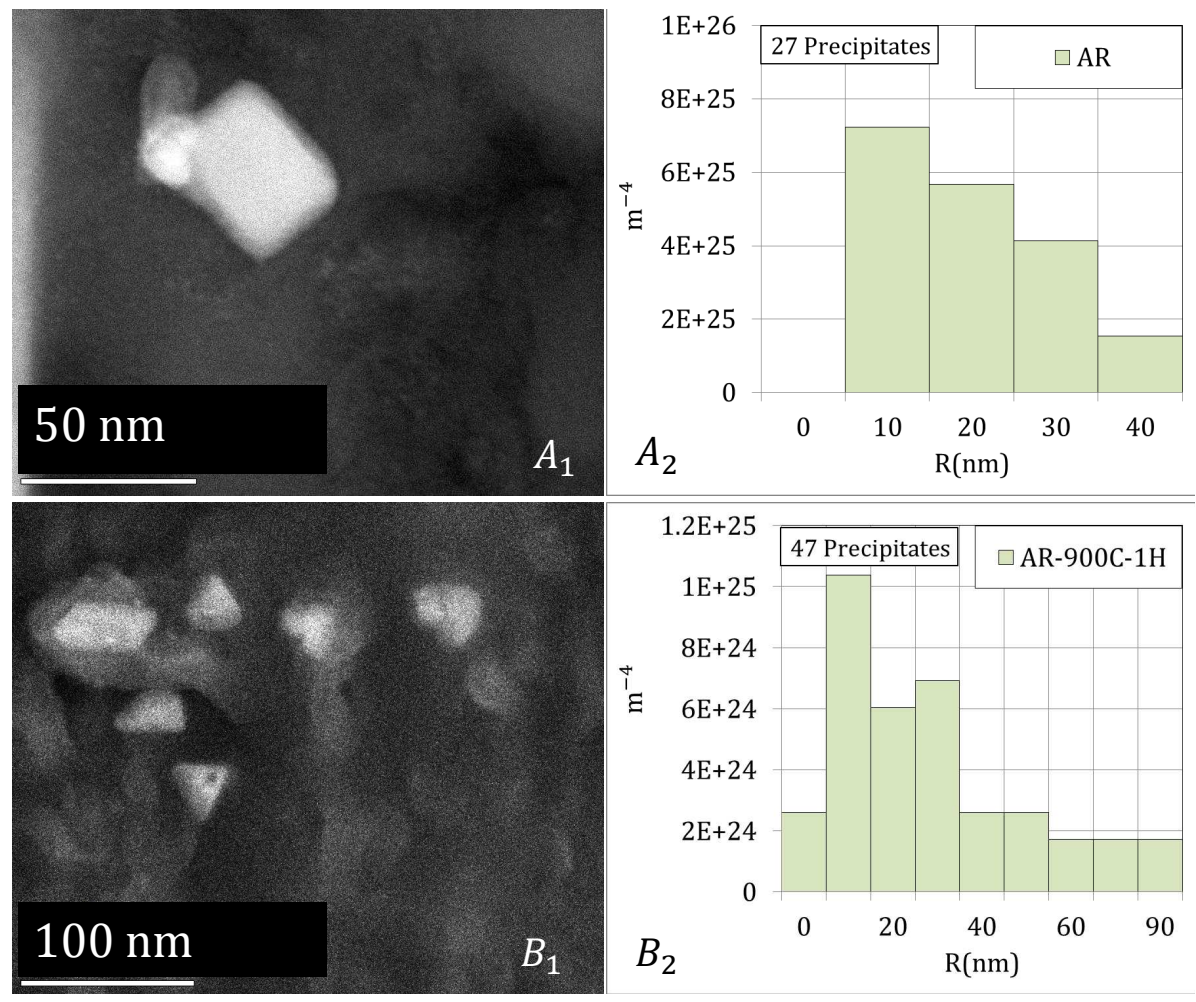

Figure 4.8: Precipitate size distribution in industrial alloy steel-A. $A_{2}$ : AR state's precipitate size distributions and $A_{1}$ : corresponding micrograph, $B_{2}$ : precipitate size distribution of $\mathrm{AR}$ state heat treated at $900^{\circ} \mathrm{C}$ for $1 \mathrm{hr}$ and $B_{1}$ : corresponding micrograph. 

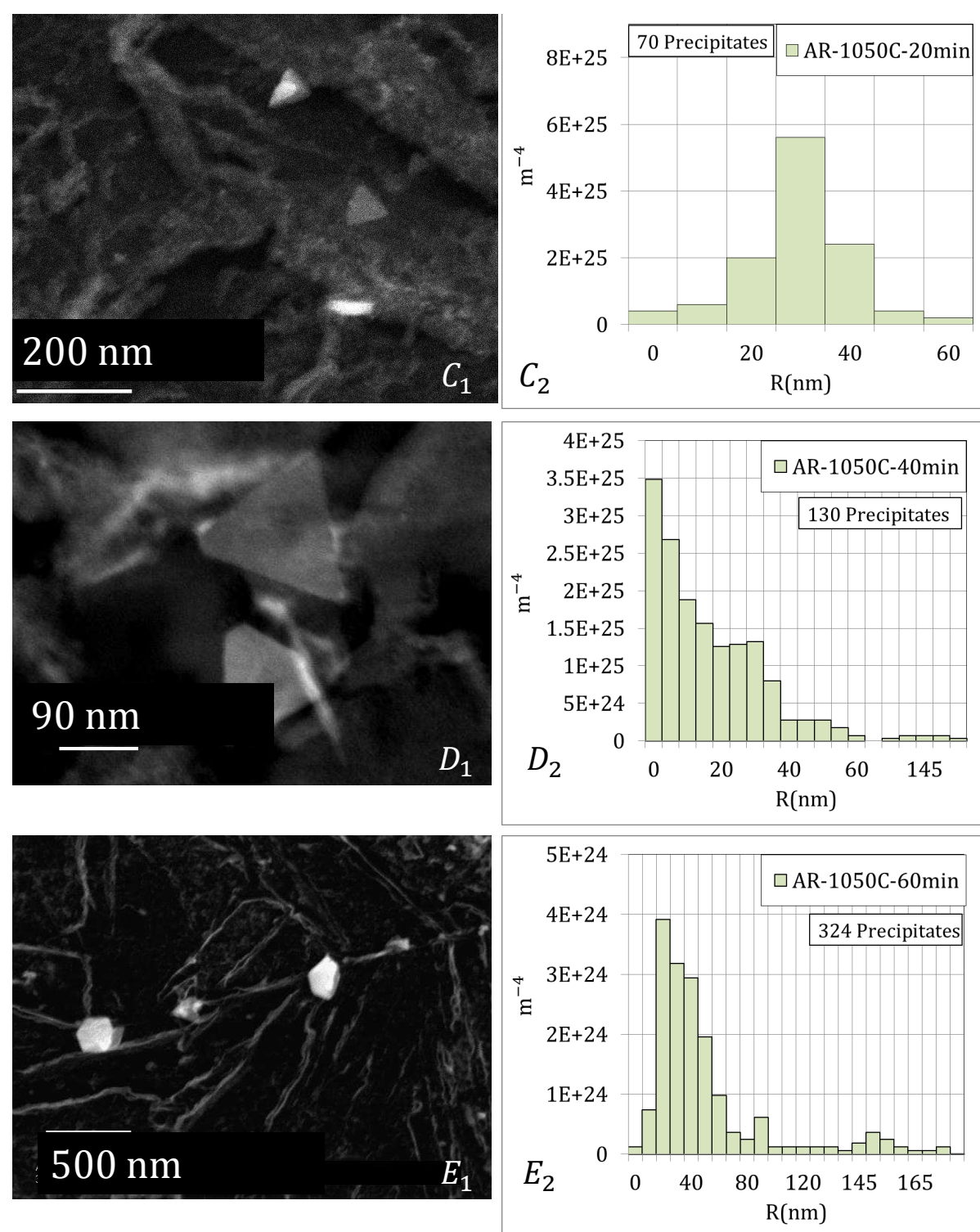

Figure 4.9: Precipitate size distribution in industrial alloy steel-A. $C_{2}$ : precipitate size distribution of $\mathrm{AR}$ state heat treated at $1050^{\circ} \mathrm{C}$ for $20 \mathrm{~min}$ and $C_{1}$ : corresponding micrograph, $D_{2}$ : precipitate size distribution of AR state heat treated at $1050^{\circ} \mathrm{C}$ for $40 \mathrm{~min}$ and $D_{1}$ : corresponding micrograph and $E_{2}$ : precipitate size distribution of $\mathrm{AR}$ state heat treated at $1050^{\circ} \mathrm{C}$ for 60 min and $E_{1}$ : corresponding micrograph. 

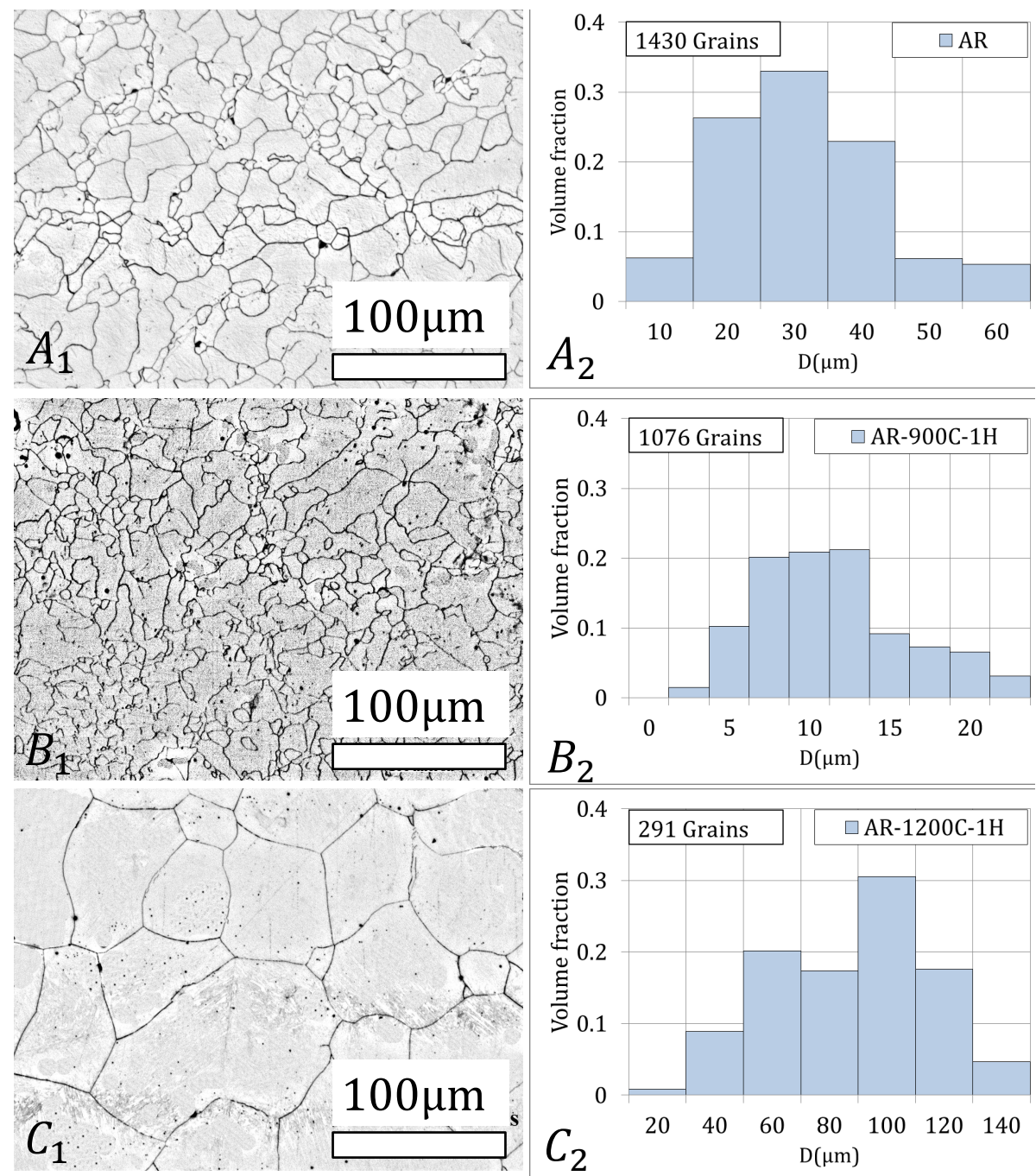

Figure 4.10: Grain size distribution in industrial alloy steel-A. $A_{2}$ : AR state's grain size distributions and $A_{1}$ : Corresponding micrograph, $B_{2}$ : grain size distribution of AR state heat treated at $900^{\circ} \mathrm{C}$ for $1 \mathrm{hr}$ and $B_{1}$ : corresponding micrograph and $C_{2}$ : grain size distribution of $\mathrm{AR}$ state heat treated at $1200^{\circ} \mathrm{C}$ for $1 \mathrm{hr}$ and $C_{1}$ : Corresponding micrograph. 

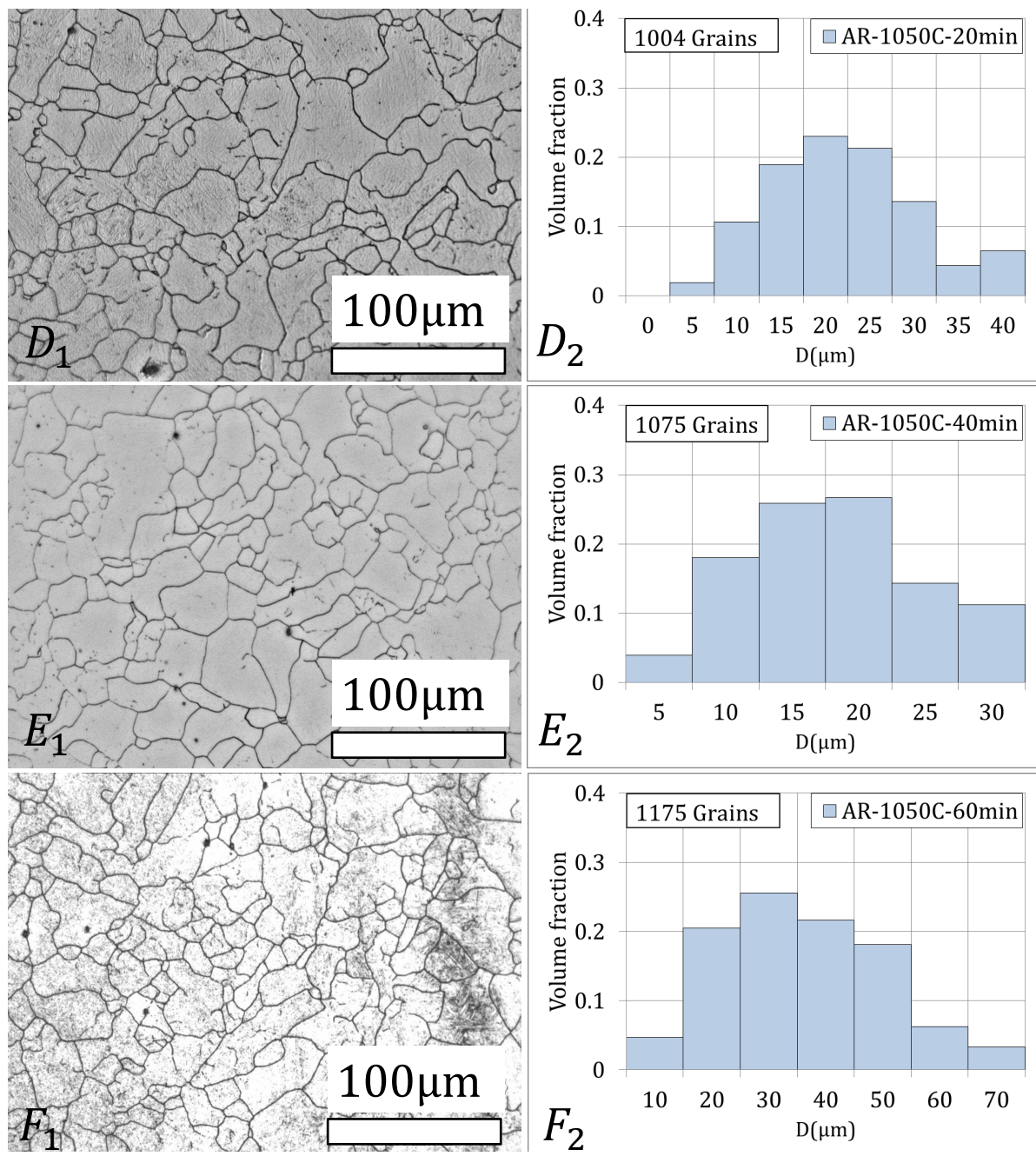

Figure 4.11: Grain size distribution in industrial alloy steel-A. $D_{2}$ : grain size distribution of $\mathrm{AR}$ state heat treated at $1050^{\circ} \mathrm{C}$ for $20 \mathrm{~min}$ and $D_{1}$ : Corresponding micrograph, $E_{2}$ : grain size distribution of $\mathrm{AR}$ state heat treated at $1050^{\circ} \mathrm{C}$ for 40 min and $E_{1}$ : corresponding micrograph and $F_{2}$ : grain size distribution of AR state heat treated at $1050^{\circ} \mathrm{C}$ for $60 \mathrm{~min}$ and $F_{1}$ : Corresponding micrograph. 


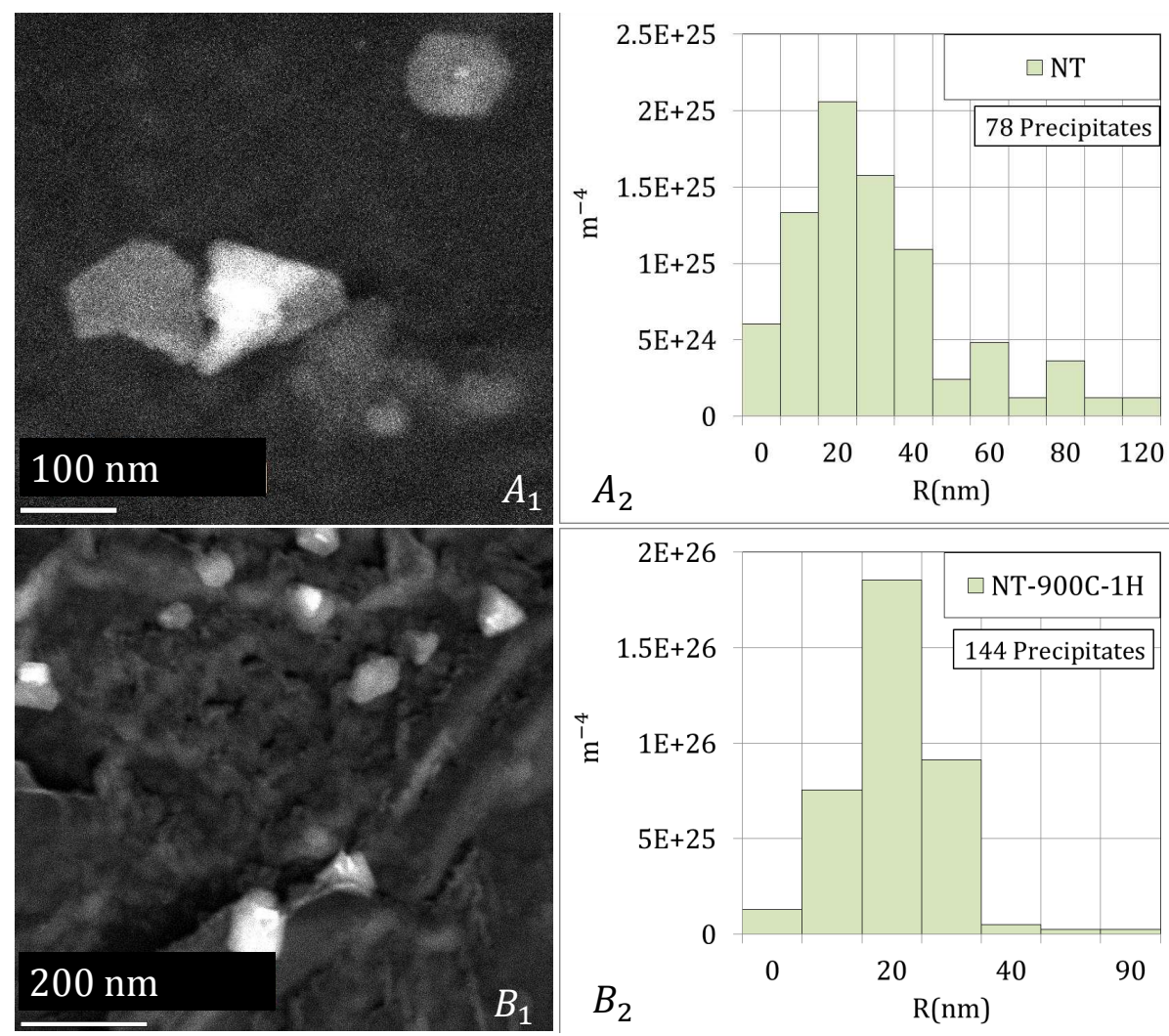

Figure 4.12: Precipitate size distribution in industrial alloy steel-A. $A_{2}$ : NT state's precipitate size distributions and $A_{1}$ : Corresponding micrograph, $B_{2}$ : precipitate size distribution of NT state heat treated at $900^{\circ} \mathrm{C}$ for $1 \mathrm{hr}$ and $B_{1}$ : corresponding micrograph.

treatments, AR state showed normal grain growth.

In figure 4.12 and 4.13, the NT state's precipitate size distributions with corresponding micrographs are presented. Figure $4.12\left(A_{2}\right)$ and $\left(B_{2}\right)$ represent precipitate size distribution in the NT and NT state heat treated at $900^{\circ} \mathrm{C}$ for 1 hr. Similarly figure 4.13, shows the precipitate size distributions in NT state heat treated at $1050^{\circ} \mathrm{C}$ for 20,40 and $60 \mathrm{~min}$. There is an increase in distribution width with increasing holding time at $1050^{\circ} \mathrm{C}$. Unlike in the AR state, in the NT state a lognormal precipitate size distribution is obtained in $1050^{\circ} \mathrm{C}-60$ min heat treatment.

Prior austenite grain size distribution of the NT state heat treated at different temperatures are shown in figure 4.14 and 4.15. From figure 4.14, it can be seen that the prior austenite grain size distribution remained quasi-stationary (normal growth) for heat treatment done at $900^{\circ} \mathrm{C}$ and $1200^{\circ} \mathrm{C}$ for $1 \mathrm{hr}$. As stated in the case of AR state, at $1200^{\circ} \mathrm{C}$ the grain size is comparatively large as all the precipitates are dissolved in the matrix and austenite grains can grow without any hinderance. In figure 4.15 , the prior austenite grain size distributions of NT state heat treated 

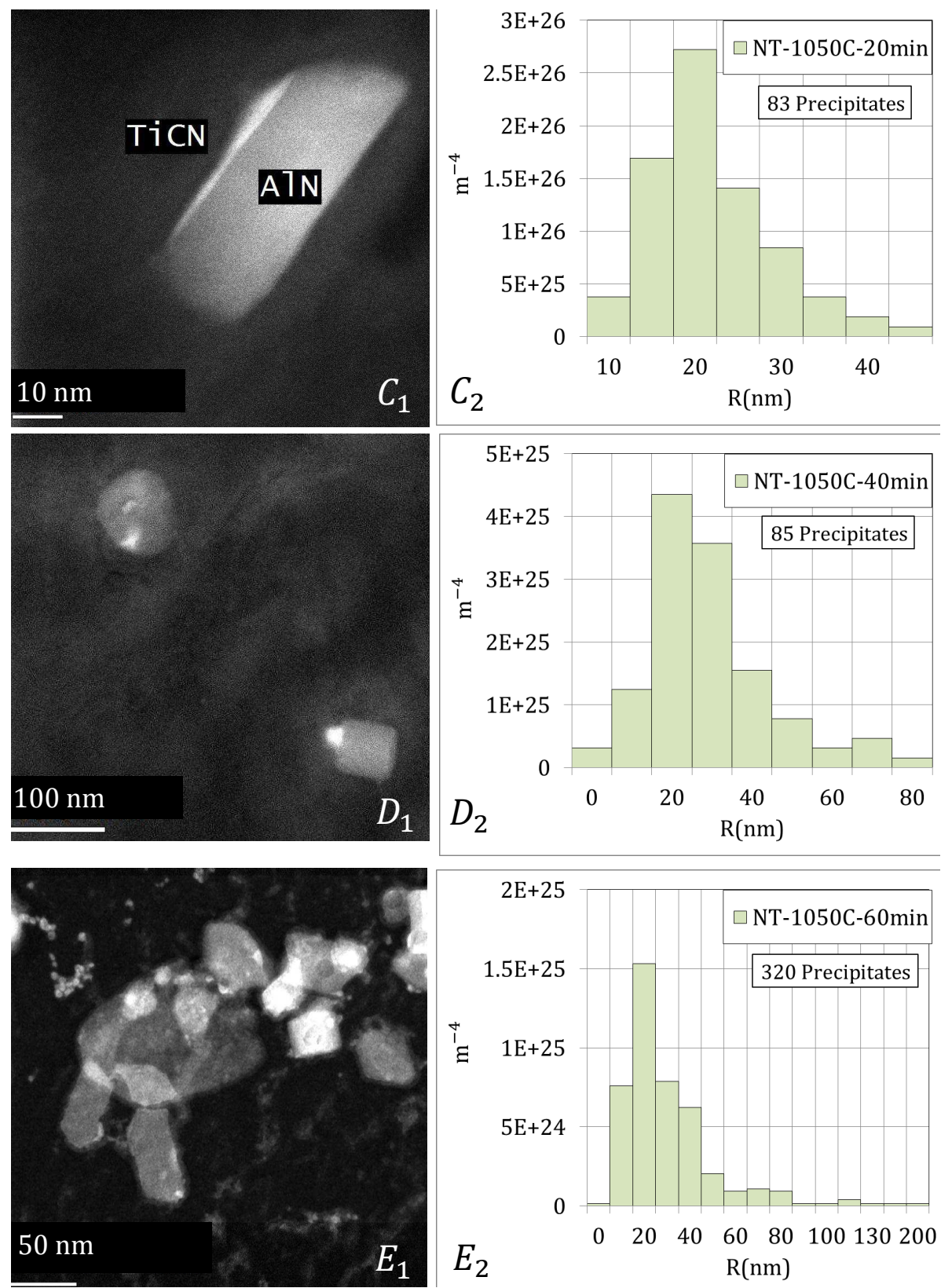

Figure 4.13: Precipitate size distribution in industrial alloy steel-A. $C_{2}$ : precipitate size distribution of $\mathrm{NT}$ state heat treated at $1050^{\circ} \mathrm{C}$ for $20 \mathrm{~min}$ and $C_{1}$ : Corresponding micrograph, $D_{2}$ : precipitate size distribution of NT state heat treated at $1050^{\circ} \mathrm{C}$ for $40 \mathrm{~min}$ and $D_{1}$ : corresponding micrograph and $E_{2}$ : precipitate size distribution of $\mathrm{NT}$ state heat treated at $1050^{\circ} \mathrm{C}$ for $60 \mathrm{~min}$ and $E_{1}$ : corresponding micrograph. 


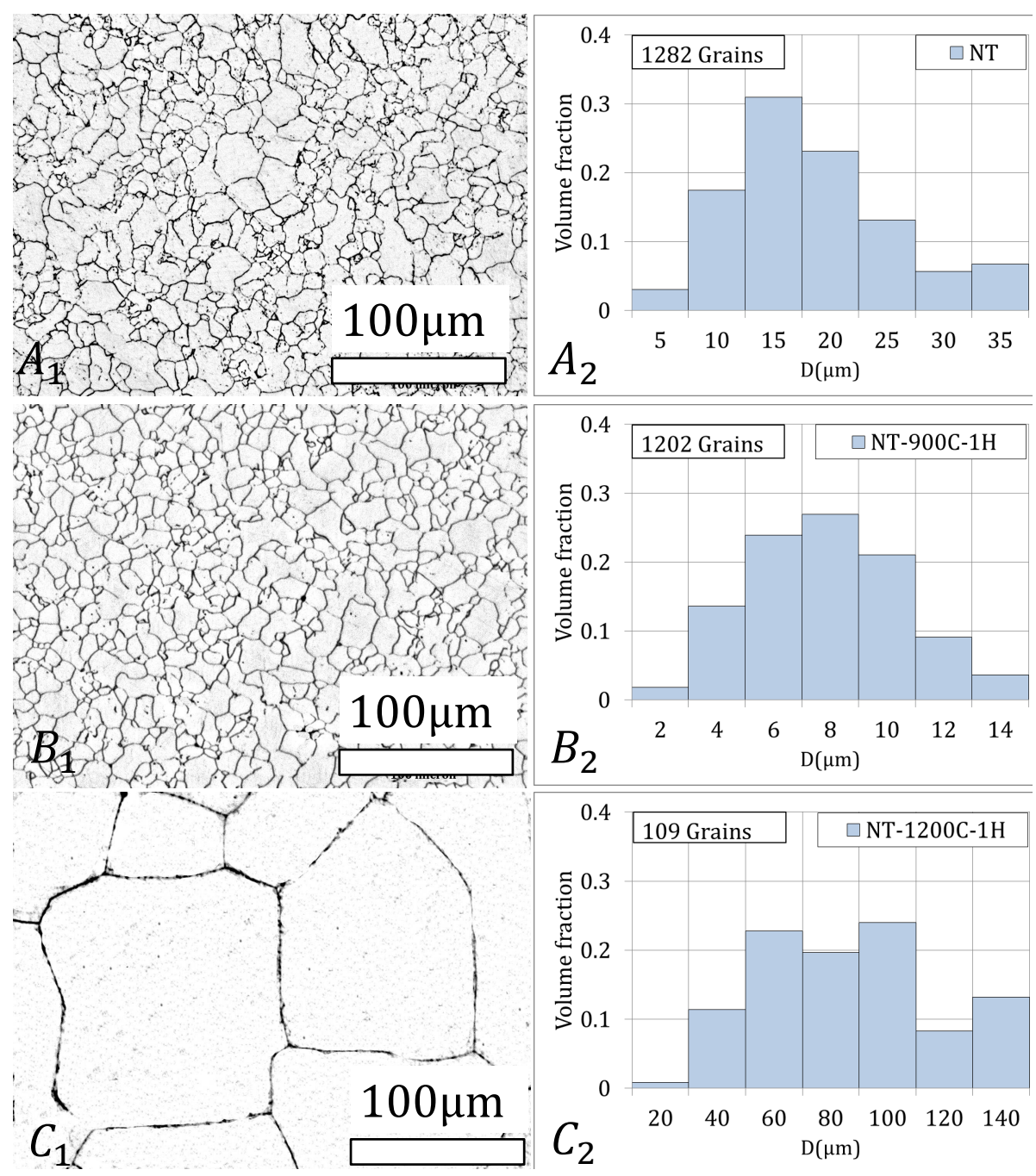

Figure 4.14: Grain size distribution in industrial alloy steel-A. $A_{2}$ : NT state's grain size distributions and $A_{1}$ : Corresponding micrograph, $B_{2}$ : grain size distribution of NT state heat treated at $900^{\circ} \mathrm{C}$ for $1 \mathrm{hr}$ and $B_{1}$ : corresponding micrograph and $C_{2}$ : grain size distribution of $\mathrm{NT}$ state heat treated at $1200^{\circ} \mathrm{C}$ for $1 \mathrm{hr}$ and $C_{1}$ : Corresponding micrograph. 

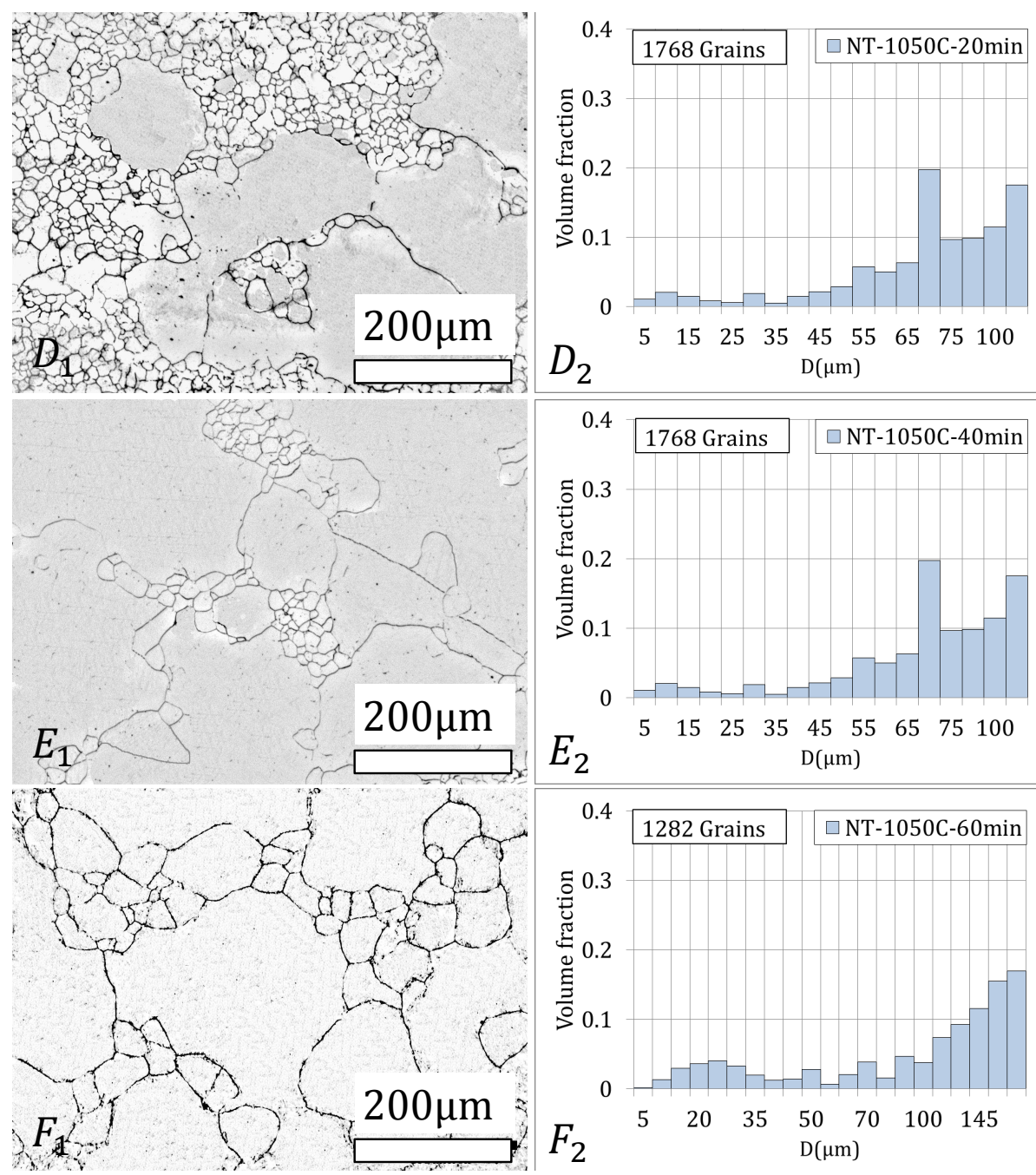

Figure 4.15: Grain size distribution in industrial alloy steel-A. $D_{2}$ : grain size distribution of NT state heat treated at $1050^{\circ} \mathrm{C}$ for $20 \mathrm{~min}$ and $D_{1}$ : Corresponding micrograph, $E_{2}$ : grain size distribution of $\mathrm{NT}$ state heat treated at $1050^{\circ} \mathrm{C}$ for 40 min and $E_{1}$ : corresponding micrograph and $F_{2}$ : grain size distribution of NT state heat treated at $1050^{\circ} \mathrm{C}$ for $60 \mathrm{~min}$ and $F_{1}$ : Corresponding micrograph. 


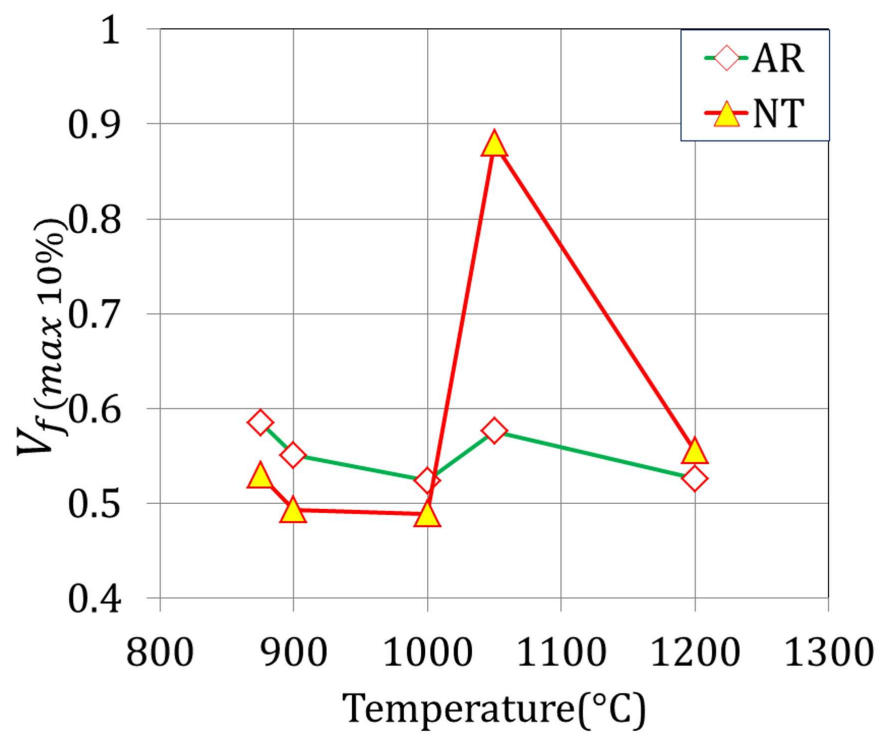

Figure 4.16: Cumulative volume fraction of largest $10 \%$ grains by number of the $\mathrm{AR}$ and NT states heat treated at different temperatures.

at $1050^{\circ} \mathrm{C}$ for 20,40 and $60 \mathrm{~min}$ are presented. All the grain size distributions along with the adjacent micrographs show that the grain growth at this stage is Abnormal Grain Growth (AGG). From figure $4.15\left(D_{2}\right),\left(E_{2}\right)$ and $\left(F_{2}\right)$, it can be seen that the largest grains volume fraction is higher than the smaller ones. Thus the largest grains in the matrix occupying most of the volume in the matrix which is a known identifier of AGG microstructure.

In figure 4.16, the largest $10 \%$ grains (by number) cumulative volume fraction is shown for both AR and NT state. It can be seen from the figure that after an $1 \mathrm{hr}$ heat treatment at $1050^{\circ} \mathrm{C}$ of the NT state, the largest grains are occupying $90 \%$ of the total volume. So, these grains are occupying major fraction of the total volume which is a confirmation of AGG in microstructure. From figures 4.14, 4.15 and 4.16 it can be said that the NT state heat treated at $1050^{\circ} \mathrm{C}$ for different times (20, 40, 60 min) showed AGG while the AR state showed NGG.

\subsubsection{Model prediction}

The soft coupled model results for the industrial alloy steel-A is shown in figure 4.17. It can be seen that the model is predicting that there is no probability of AGG in the AR state heat treated at $900^{\circ} \mathrm{C}, 1050^{\circ} \mathrm{C}$ and $1200^{\circ} \mathrm{C}\left(d / d t\left(D_{a b} / D_{n}\right)=0\right.$ so $\left.D_{a b}^{\max } / D_{n}=0\right)$. On the other hand, the model predicts that there is a probability of AGG in the NT state heat treated at $900^{\circ} \mathrm{C}$. As the maximum grain size $\left(D_{a b}^{\max }\right)$ is about 2 times of the mean grain size $\left(D_{n}\right)$, the system might experience AGG. Although the precipitation state presents a condition suitable for AGG, grain boundary mobility at $900^{\circ} \mathrm{C}$ might not allow explosive growth of the larger grains 


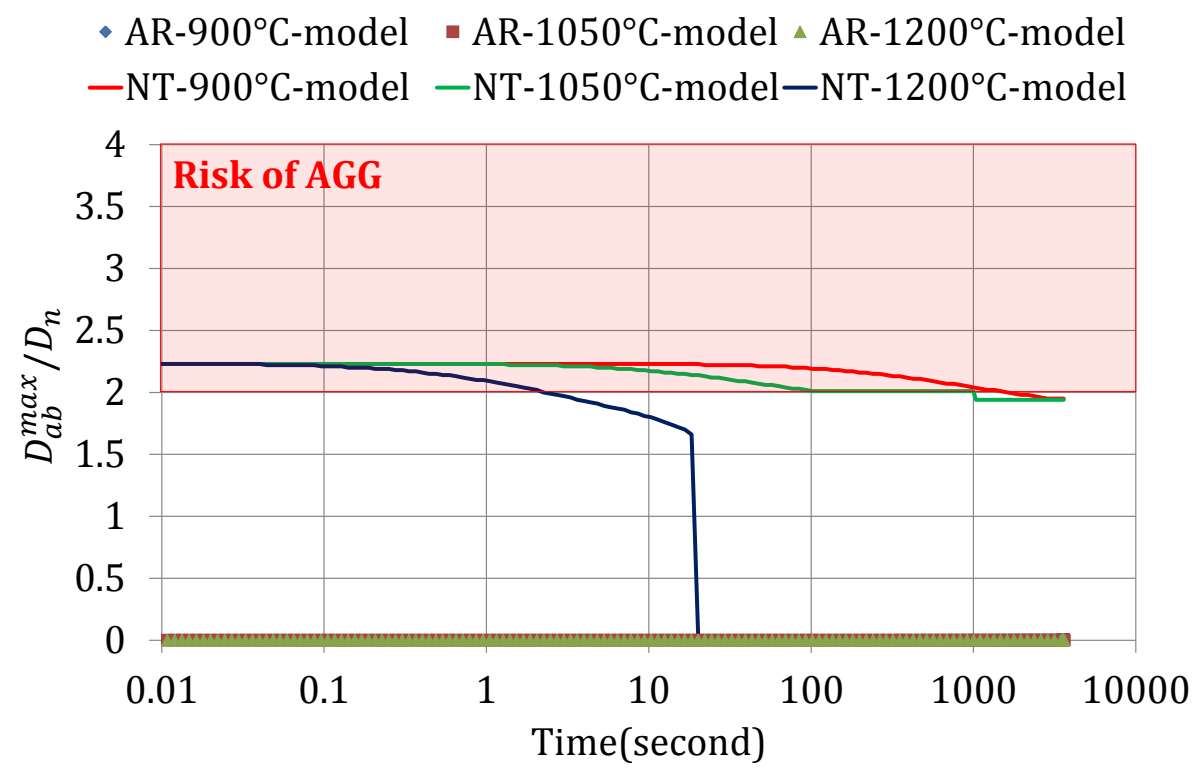

Figure 4.17: Soft coupled model prediction of the industrial alloy steel-A's As Rolled (AR) and Normalized Tempered (NT) state. The red shaded zone mark the risk of AGG for $D_{a b}^{\max } / D_{n}$ value. Here, the model parameters $\mu=0.54$ and $\lambda=0.6$.

in the distribution to occur. It should be narrated again here that the mean grain size evolution is not considered in this modeling approach.

The model prediction at $1050^{\circ} \mathrm{C}$ for the NT state suggests that there is a possibility of AGG occurrence $\left(D_{a b}^{\max } / D_{n}>2\right)$ till $\sim 1000$ sec into the heat treatment. The model prediction is in agreement with the experimental observation (see figure 4.15). At $1050^{\circ} \mathrm{C}$ the grain boundary mobility can be assumed to be sufficiently high to allow explosive growth to occur thus AGG is probable. The model results showed that AGG is probable, at the beginning of the $1200^{\circ} \mathrm{C}$ heat treatment but eventually disappear above $10 \mathrm{sec}$ into the heat treatment. A very short period ( $10 \mathrm{sec}$ ) of exposure to AGG condition and comparatively longer period of NGG condition exposure of the microstructure yield NGG at the end of the heat treatment. Summary of the all the experimental observations and model predictions of grain growth condition are listed in the table 4.2 .

\subsubsection{Industrial alloy: steel-B}

\subsubsection{Composition and heat treatment}

Composition of the industrial alloy steel-B is given in table 5.4. There are two major precipitating elements $\mathrm{Nb}$ and $\mathrm{Al}$ in the alloy. TEM and also ICP analysis confirmed the presence of $\mathrm{NbC}$ and $\mathrm{AlN}$ precipitates in the different heat treated states (for details see appendix A). 
Table 4.2: Steel-A: a summary of the model predictions and experimental observations of grain growth condition.

\begin{tabular}{|c|c|c|}
\hline State & Model prediction & Experimental observation \\
\hline AR- $900^{\circ} \mathrm{C}(1 \mathrm{hr})$ & NGG & NGG \\
\hline AR- $1050^{\circ} \mathrm{C}(1 \mathrm{hr})$ & NGG & NGG \\
\hline AR- $-1200^{\circ} \mathrm{C}(1 \mathrm{hr})$ & NGG & NGG \\
\hline NT- $900^{\circ} \mathrm{C}(1 \mathrm{hr})$ & AGG & NGG \\
\hline NT $-1050^{\circ} \mathrm{C}(1 \mathrm{hr})$ & AGG & AGG \\
\hline NT- $-1200^{\circ} \mathrm{C}(1 \mathrm{hr})$ & NGG & NGG \\
\hline
\end{tabular}

Table 4.3: Composition of industrial alloy steel-B in atomic $\%$

\begin{tabular}{cccccccc}
\hline $\mathrm{C}$ & $\mathrm{Si}$ & $\mathrm{Mn}$ & $\mathrm{Ni}$ & $\mathrm{Cr}$ & $\mathrm{Al}$ & $\mathrm{Nb}$ & $\mathrm{N}$ \\
0.0963 & 0.352 & 1.22 & 0.133 & 1.23 & 0.0701 & 0.0221 & 0.0929 \\
\hline
\end{tabular}

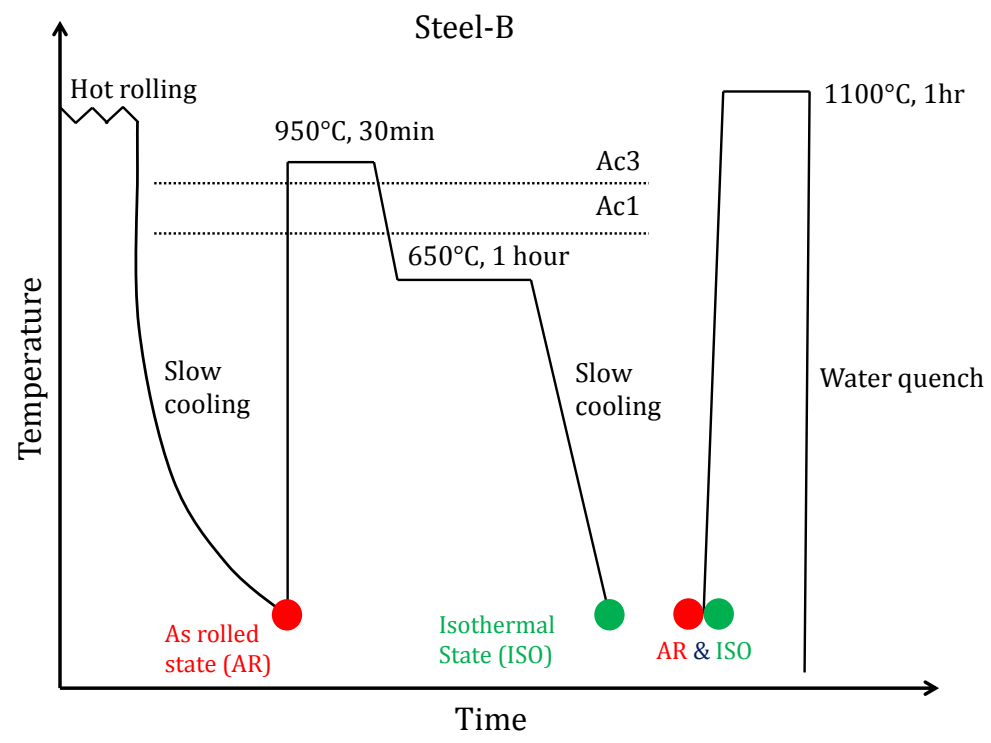

Figure 4.18: Heat treatment cycle performed on the alloy steel-B to obtain the As Rolled (AR) and Isothermally heat treated (ISO) state. 

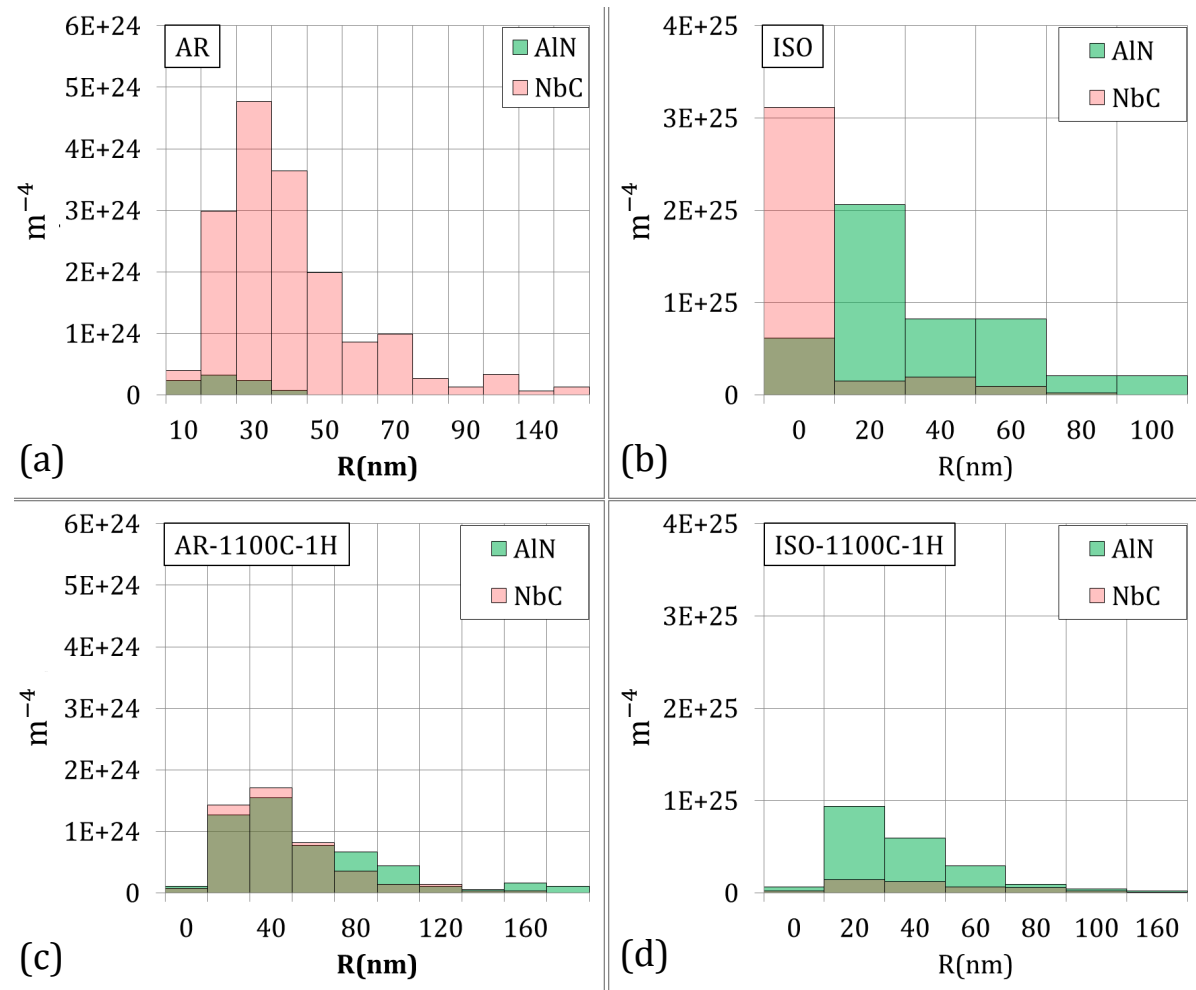

Figure 4.19: AlN and NbC precipitate size distribution in the industrial alloy steelB. (a) AR state, (c) AR state heat treated at $1100^{\circ} \mathrm{C}$ for $1 \mathrm{hr}$, (b) ISO state and (d) ISO state heat treated at $1100^{\circ} \mathrm{C}$ for $1 \mathrm{hr}$.

Two states of the steel-B are studied: (1) As Rolled (AR) and (2) Isothermally heat treated state (ISO). The AR state is obtained by rolling of the as cast section followed by a slow air cooling, while the ISO state is obtained by an isothermal heat treatment above $\left(A C_{3}\right)$ and below $\left(A C_{1}\right)$ the austenite transformation temperatures (see figure 4.18). A slow cooling followed by the rolling insured a fully recrystallized microstructure. An isothermal heat treatment of the AR state is done to obtain a fully precipitated state. Subsequent austenitizing treatments are performed on the AR and ISO states to observe precipitation state influence on austenite grain growth condition (shown in the figure 4.18).

\subsubsection{Precipitation state and grain size evolution}

Figure 4.19 represents precipitate size distributions of the AR and ISO states. In the $\mathrm{AR}$ state, $\mathrm{NbC}$ has a higher volume fraction in comparison with AlN. After the heat treatment at $1100^{\circ} \mathrm{C}$ for $1 \mathrm{hr}$, both $\mathrm{AlN}$ and $\mathrm{NbC}$ show similar volume fraction (see figure 4.19(c)). ISO state has a higher volume fraction of both $\mathrm{NbC}$ and AlN precipitates in comparison with the AR state. Subsequent heat treatment of the ISO state at $1100^{\circ} \mathrm{C}$ leads to a similar $\mathrm{AlN}$ and $\mathrm{NbC}$ volume fractions.

Prior austenite grain size distributions of the AR and ISO states are presented in 

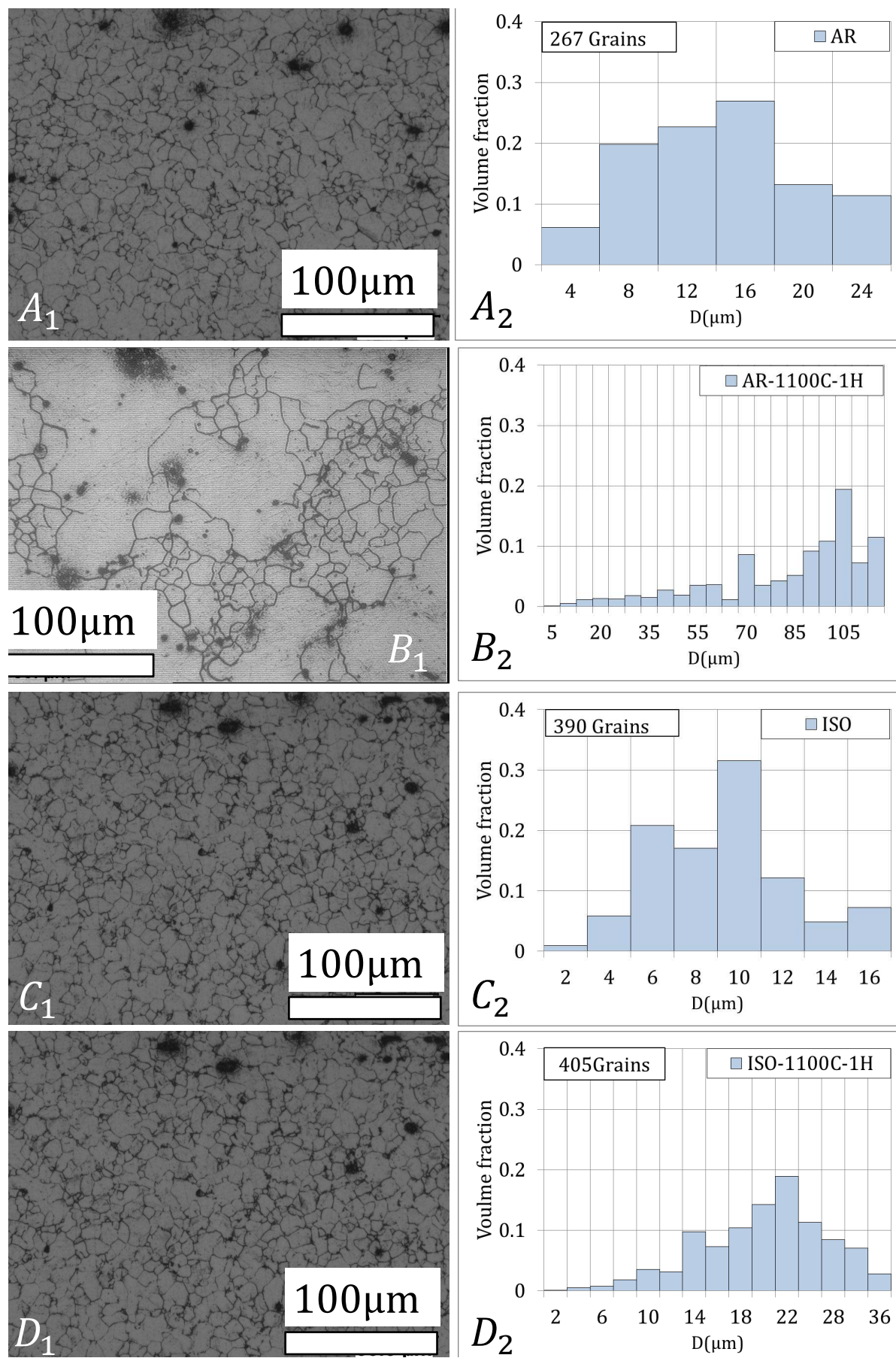

Figure 4.20: Grain size distribution in the industrial alloy steel-B. $A_{2}$ : the AR state's grain size distributions and $A_{1}$ : corresponding micrograph, $B_{2}$ : grain size distribution of the AR state heat treated at $1100^{\circ} \mathrm{C}$ for $1 \mathrm{hr}$ and $B_{1}$ : corresponding micrograph, $C_{2}$ : the ISO state's grain size distributions and $C_{1}$ : corresponding micrograph and $D_{2}$ : grain size distribution of the ISO state heat treated at $1100^{\circ} \mathrm{C}$ for $1 \mathrm{hr}$ and $D_{1}$ : corresponding micrograph. 


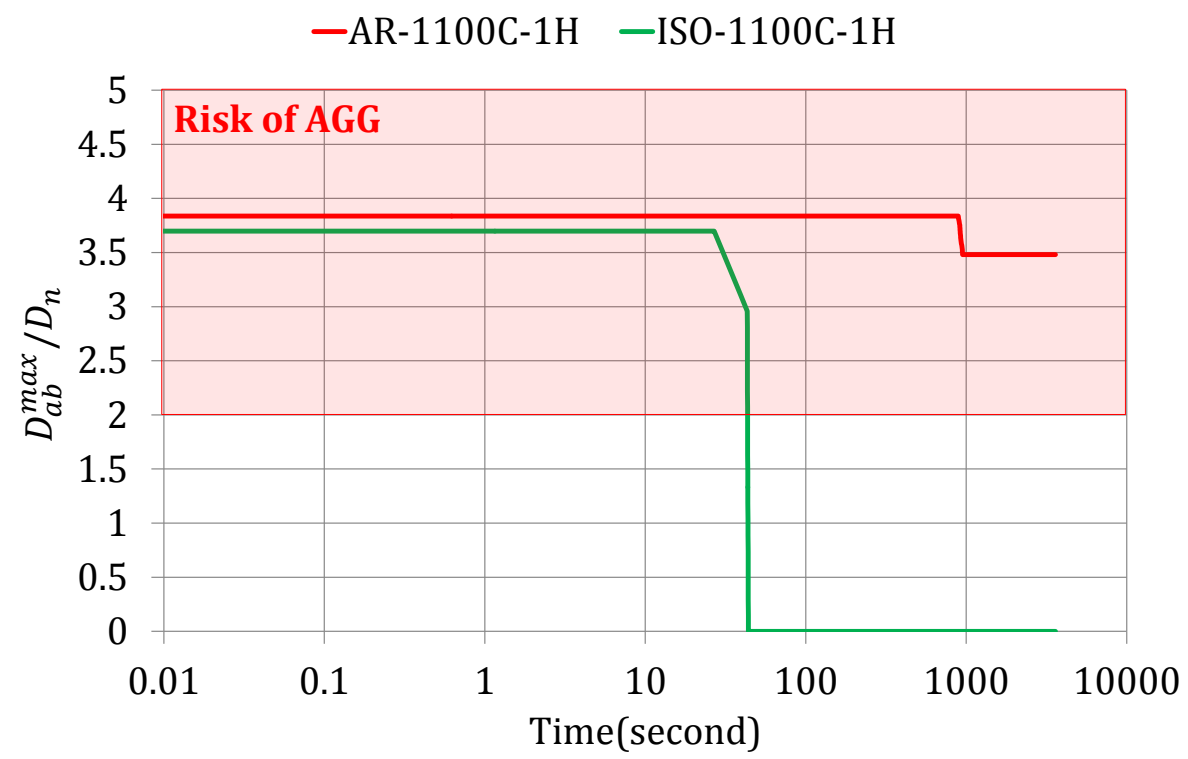

Figure 4.21: Soft coupled model prediction for industrial alloy steel-B's AR and ISO state. The red shaded zone mark the risk of AGG area for $D_{a b}^{\max } / D_{n}$ value. Here, model parameters $\mu=0.54$ and $\lambda=0.6$.

figure 4.20. From figure 4.20, it can be observed that only the AR state heat treated at $1100^{\circ} \mathrm{C}$ shows abnormal grain growth and all the other states have normal grain growth structure. In figure $4.20\left(B_{1}\right)$ and $\left(B_{2}\right)$, it can be seen that the larger grains are occupying major volume fraction in the microstructure. Presence of a bimodal grain size distribution (volume fraction) is a confirmation of AGG occurrence in the $\mathrm{AR}$ state heat treated at $1100^{\circ} \mathrm{C}$ for $1 \mathrm{hr}$.

\subsubsection{Model prediction}

The soft coupled model predictions for the AR and ISO state heat treated at $1100^{\circ} \mathrm{C}$ for $1 \mathrm{hr}$ is presented in figure 4.21. From the model predictions, it can be seen that the AR state has a probability of AGG from the very beginning of the heat treatment. In comparison with the microstructure presented in figure 4.20 $\left(B_{1}\right)$, the model prediction is quite in agreement with the experimental observation. The AR state heat treated at $1100^{\circ} \mathrm{C}$ for $1 \mathrm{hr}$ shows a mean austenite grain size of $\sim 16 \mu \mathrm{m}$. There is a substantial change in the mean austenite grain diameter from the initial $6 \mu \mathrm{m}$. As the mean austenite grain diameter evolution is not taken into account, the AGG condition predicted corresponds to the favorable pinning condition of AGG.

Experimental observation presented in figure $4.20\left(D_{1}\right)$ and $\left(D_{2}\right)$ shows that the ISO state does not show any AGG at $1100^{\circ} \mathrm{C}$ heat treatment. The soft coupled model predicts that there is a probability of AGG at the beginning of the heat 
Table 4.4: Steel-B: a summary of the model predictions and experimental observations of grain growth condition.

\begin{tabular}{|c|c|c|}
\hline State & Model prediction & Experimental observation \\
\hline AR- $1100^{\circ} \mathrm{C}(1 \mathrm{hr})$ & AGG & AGG \\
\hline ISO- $1100^{\circ} \mathrm{C}(1 \mathrm{hr})$ & NGG & NGG \\
\hline
\end{tabular}

treatment. The AGG probability soon disappear at $\sim 46 \mathrm{sec}$ into the heat treatment. The probability of AGG for a short period of time is not enough to obtain a fully abnormally grown microstructure. This is in agreement with the microstructure shown in figure $4.20\left(C_{1}\right)$. The ISO state heat treated at $1100^{\circ} \mathrm{C}$ for $1 \mathrm{hr}$ shows a mean austenite grain size of $\sim 9 \mu \mathrm{m}$. There is only a $3 \mu \mathrm{m}$ change in mean grain diameter from the initial $6 \mu \mathrm{m}$ at $1100^{\circ} \mathrm{C}$ after $1 \mathrm{hr}$. A Small change in mean grain diameter contributed to the effective prediction of grain growth condition. Finally, it can be concluded from the model predictions that soft coupled approach to predict AGG is effective for industrial alloy steel-B. All the experimental observations and model predictions in steel-B of grain growth condition are summarized in table 4.4 .

\subsection{Conclusions from this chapter}

In this chapter, a soft coupled modeling approach of precipitation and abnormal grain growth model is presented. The modeling scheme is presented in details. The model prediction sensitivity to different precipitation state evolution in low alloy steel is also studied. The model is tested for two industrial alloys steel-A and steel-B and comparison with experimental observations are presented. The main conclusions that can be obtained from this chapter are summarize below:

- Soft coupling of precipitation and abnormal grain growth model follow the unidirectional data flow method. Here, it is assumed that precipitate nucleation and growth is homogenous. Computed data from the precipitation model is fed into the abnormal grain growth model to predict normal and abnormal grain growth conditions.

- Simplicity in the soft coupled model is kept by assuming that the mean austenite grain size remains constant throughout the heat treatment.

- A theoretical study using three precipitation state evolutions such as: (1) AGG in presence of precipitate coarsening, (2) AGG in presence of precipitate nucleation and (3) AGG in presence of precipitate dissolution gave the following understandings:

1. Decrease in precipitate number density (constant volume fraction), de- 
creases the AGG probability.

2. A certain quantity of precipitate volume fraction and number density is required for $A G G$ to occur.

3. The AGG probability disappear with decreasing precipitate volume fraction and number density (dissolution).

- It is observed that for the industrial alloy steel-A, the model prediction is quite in agreement with the experimental observations for different heat treated states.

- In the case of industrial alloy steel-B, the model prediction is also in agreement for both the As Rolled and isothermally heat treated states.

- One of the most important findings from the soft coupled modeling is that the initial austenite grain size plays an important role in determining the austenite grain growth conditions.

- The present scheme of soft coupled model is quite effective in predicting AGG in low alloy steel, but there can be improvements that need to be considered:

1. The mean grain diameter $\left(D_{n}\right)$ during holding at a particular austenitizing temperature does not remain constant. It is already shown that mean grain size is a key factor in the AGG initiation. So, evolution of mean grain size can induce changes in AGG or NGG conditions which needs to be taken into account.

2. The knowledge of $D_{n}(t)$, can provide further possibilities to incorporate mechanical properties modeling.

So, it is necessary to account for mean grain size evolution with time. This is done in the next chapter with a dynamic coupling between the precipitation and abnormal grain growth model. 
Cette thèse est accessible à l'adresse : http://theses.insa-lyon.fr/publication/2013ISAL0094/these.pdf () [M.A. Razzak], [2013], INSA de Lyon, tous droits réservés 


\section{Chapter 5}

\section{Dynamic coupled modeling of precipitation and grain growth}

In this chapter, influences of precipitation state evolution on abnormal/normal grain growth is presented by coupling between precipitation (see chapter 3) and Abnormal Grain Growth (AGG) (see chapter 2) model dynamically. Dynamic coupling is defined by the coupled modeling approach considering the interaction between mean grain growth and precipitation state evolution while predicting the grain growth condition. Here the term 'Dynamic Coupled Modeling (DCM)' is used to emphasize that the precipitation model and AGG model is executed simultaneously on the same time scale. Significant improvement of DCM over the "Soft Coupled Model (SCM)' (see chapter 4) is the mean grain size evolution calculation. In the AGG model, it is assumed that a pre-existing large grain $D_{a b}$ is surrounded by mean sized grains $D_{n}$. Driving energy (decrease in interfacial energy) of AGG solely depends on the number of available grains surrounding it. As the number of surrounding grains depend on $D_{n}$, the evolution in mean grain size effectively reduces the AGG driving energy. Besides the change in AGG driving energy, number of corner points in a microstructure also depends on $D_{n}$. An increase in mean grain size $D_{n}$ decreases the number of corner points and consequently corner point pinning pressure. Zener pinning remains unchanged, as it is not dependent on mean grain size. So, a change in $D_{n}$ effects both the AGG driving energy and total pinning, inevitably the AGG probability. Evolution of mean grain size rather portray a complex system with interconnected physical variables. The DCM objective is to render the mutual relationship between precipitation state evolution and grain growth conditions as a function of time and temperature. In the following, a short recall of some of the previous works is given.

There are previous works regarding coupled modeling of precipitation and grain growth $[5,44,42,69]$ but very few of them dealt with the AGG prediction. Among 
the available works, Andersen et. al. [5] proposed a coupled modeling approach for the abnormal grain growth and precipitate evolution. The precipitate stability is modeled using the solubility product and concurrently the precipitate growth or coarsening is calculated using two different expressions. The biggest limitation of this approach is that the precipitate nucleation is not considered, although it can change both the volume fraction and mean radius and consequently the pinning.

M. Militzer et al [44] dealt with the effects of pinning on prior austenite grain growth by fitting the experimental grain size data to an empirical power law. They introduced a statistical grain growth model based on Abbruzzese and Luc̈ke [1] proposition. The precipitate induced pinning is expressed as a parameter (pinning parameter) which vary linearly with temperature. Their proposed method is applicable to known alloy where substantial experimental data is available. A fitted pinning parameter constricts the understanding of different aspects effecting the pinning conditions evolution and also grain growth condition itself.

David San Martín et al [42] also proposed a coupled model of precipitation and grain growth, quite similar to Andersen et. al. [5]. In their work, prior austenite grain size is modeled considering Zener pinning in the case of normal grain growth. Like Andersen et. al. [5], this work is also limited by only incorporating precipitate mean radius evolution. Precipitate nucleation is not proposed in their coupled approach to model the effects of nucleating precipitate on grain growth. Besides that additional model parameters are required to model the non-isothermal heating rate effects on grain grain growth.

$\mathrm{Xu}$ K. et al [69] presented a model concerning austenite grain size evolution during continuous casting. In their approach, a CNGTs based multi class precipitation model is implemented to predict the precipitation state evolution. In the mean time, precipitation state prediction is utilized in a grain growth model to measure the pinning condition and mean grain size evolution. In the precipitate model the precipitate coarsening is calculated using the general population balance for particle collision (introduced by Smoluchowski [63]). On the other hand, present modeling approach calculates coarsening by the relative stability of a particular radii class (respect to $R_{k_{b} T}^{*}$ ).

It should be mentioned that apart from the above discussed literatures, there are also other works about grain growth and AGG. In the present limited frame work, the literatures that the author found most relevant with the present work are discussed.

In dynamic coupled modeling approach emphasis is given on the evolution of the mean austenite grain size and its effects on the austenite grain growth behavior. Evolution in precipitation condition change the pinning conditions. As we know precipitation induced pinning determines the mean austenite grain size which in 
turn influences AGG conditions. This interconnected behavior of precipitation state evolution and grain growth with certain complexity is studied by dynamic coupled model. Instead of analyzing separate entity of physical properties, in the current approach time and temperature dependent evolution of collective physical properties are studied.

\subsection{Model assumptions}

In the dynamic coupled approach, two models: (1) Precipitation model and (2) AGG model are dynamically coupled. The individual model assumption of (1) Precipitation model is discussed in the chapter 3 and (2) AGG model in chapter 2. Dynamic coupled modeling is based on the following assumptions:

1. In the dynamic coupled model, it is assumed that the mean grain size is evolving with time at a particular temperature. This evolution is not accounted for in the previous chapter 4 .

2. Precipitate nucleation and growth occur homogeneously.

3. Abnormal grain growth model states that the largest precipitates lie in the corner points. During grain growth, grain boundary triple points move with the grain boundaries and the precipitate located in it.

\subsection{Modeling strategy}

In the present modeling approach, a dynamic coupling between precipitation and abnormal grain growth model is chosen. The precipitation model provides prediction of the precipitate size distribution evolution at each time step. At the same time step, predicted precipitate size distribution is taken as input data in the AGG model. AGG model calculates the precipitate induced pinning and mean austenite grain growth $\left(D_{n}^{i}=D_{n}^{i-1}+d D_{n} / d t \times \Delta t\right)$.

In figure 5.1, a schematic representation of the dynamic coupled modeling strategy is given. Here, the grain growth conditions (Abnormal Grain Growth (AGG) and Normal or No Grain Growth (NGG)) are modeled assuming evolution of mean grain size $\left(D_{n}\right)$ with precipitation state. The dynamic coupled modeling approach is chosen to portrait an approximate real situation, where both precipitation state and mean grain growth effects the overall grain growth condition in a system.

A schematic representation of simulation setups is shown in figure 5.2. Isothermal heat treatment simulation (see figure 5.2 (a)) is executed starting from the target temperature at time $t_{0}=0$ sec. Here, the holding time is the difference between the end time $t_{1}$ and the starting time $t_{0}$. In the isothermal heat treatment 


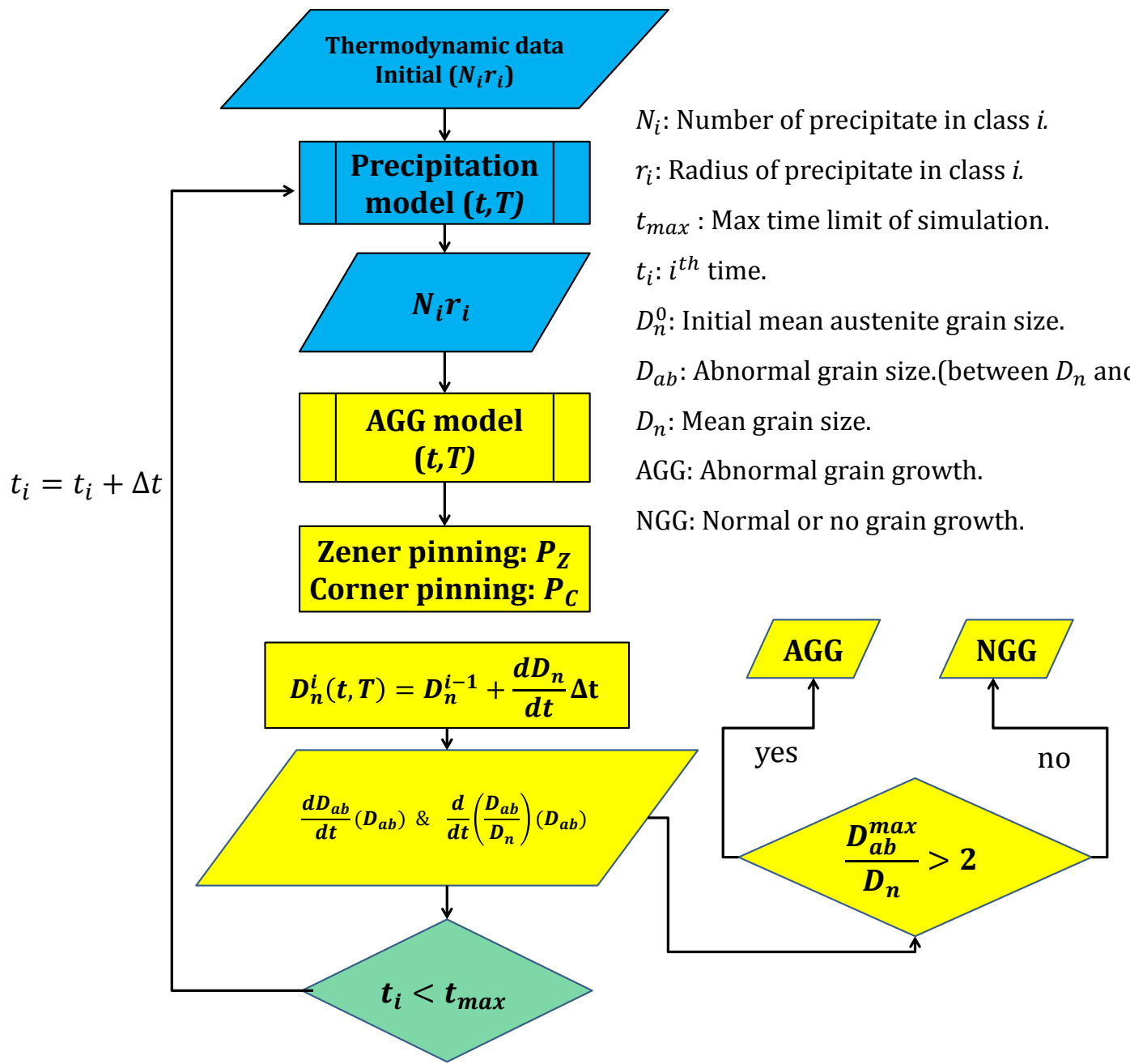

Figure 5.1: Schematic representation of dynamic coupling between precipitation and abnormal grain growth model.

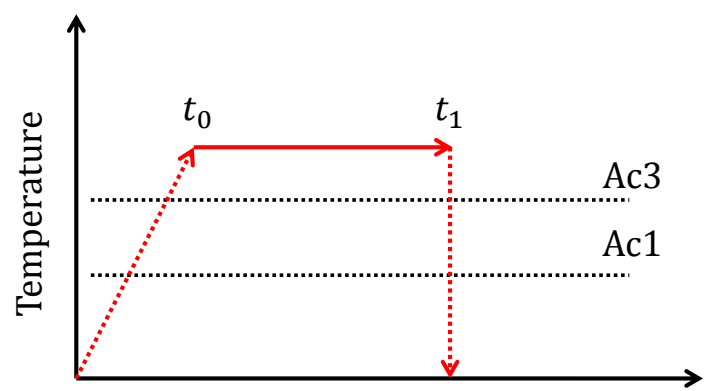

Time

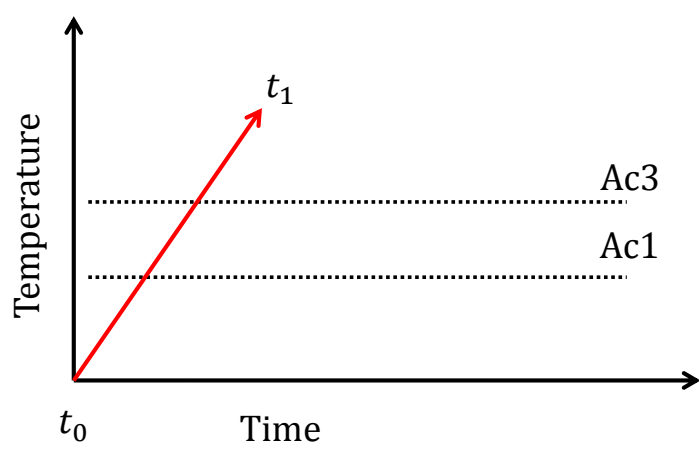

(a) Isothermal heat treatment setup

Figure 5.2: Schematic representation of simulation setup: (a) isothermal heat treatment setup and (b) heating rate simulation setup. 
simulation, the effects of heating from room temperature to the target is not considered. It is assumed that in a pre-heated furnace (to the target temperature), the heating rate is fast enough to have insignificant effects on precipitation state and austenite grain growth. Effects of heating rate is simulated (see figure 5.2 (b)) by assuming at initial time $t_{0}$, the specimen is at room temperature and at the final time $t_{1}$, it reaches target temperature. The total heating time is the difference between final time $t_{1}$ and initial $t_{0}$.

\subsubsection{Conditions for abnormal grain growth}

The abnormal grain growth model is presented in details in chapter 2. In this section, the abnormal grain growth condition is recalled briefly. As presented in chapter 2, abnormal grain growth initiation and propagation is possible if the abnormal grain growth rate is positive and also comparatively larger than the mean grain growth rate in the system. The condition for AGG is given below:

$$
\frac{d}{d t}\left(\frac{D_{a b}}{D_{n}}\right)>0
$$

The abbreviated form of the AGG condition is given below:

$$
\begin{aligned}
\frac{d}{d t}\left(\frac{D_{a b}}{D_{n}}\right) & =\frac{1}{D_{n}} \frac{d D_{a b}}{d t}-\frac{D_{a b}}{D_{n}^{2}} \frac{d D_{n}}{d t} \\
& =\frac{2 \gamma_{g} M}{D_{n}^{2}}\left[\mu-(1-\mu)\left(\frac{D_{n}}{D_{a b}}\right)^{2}+(2 \mu-1) \frac{D_{n}}{D_{a b}}-\frac{\lambda D_{a b}}{2 D_{n}}\right] \\
& -\frac{M}{D_{n}}\left(\frac{D_{a b}}{D_{n}}+1\right)\left(P_{C}+P_{Z}\right)
\end{aligned}
$$

Here, $P_{Z}$ is the Zener pinning and $P_{C}$ is the corner pinning.

$$
M=M_{0} \exp \left(-\frac{Q}{R T}\right)
$$

Here, $D_{a b}$ can vary between $D_{n}$ and $\infty$. Austenite grain boundary mobility $M$ is expressed in equation 5.3 , where $M_{0}=3.16 \times 10^{-2} \mathrm{~m}^{2} / \mathrm{s}$ is the pre-exponential factor, $Q=300 \mathrm{~kJ} / \mathrm{mol}$ is the grain boundary activation energy [45], $R$ is the gas constant and $T$ is the temperature in Kelvin.

The expression of AGG condition 5.2 is in quadratic form. There are two values of $D_{a b}$ delimiting a domain within which the AGG condition is fulfilled. The smallest value of $D_{a b}$ can be denoted as $D_{a b}^{\min }$ and the largest as $D_{a b}^{\max }$ (see figure 5.3). $D_{a b}^{\min }$ and $D_{a b}^{\max }$ is representing the range of grains in a distribution 


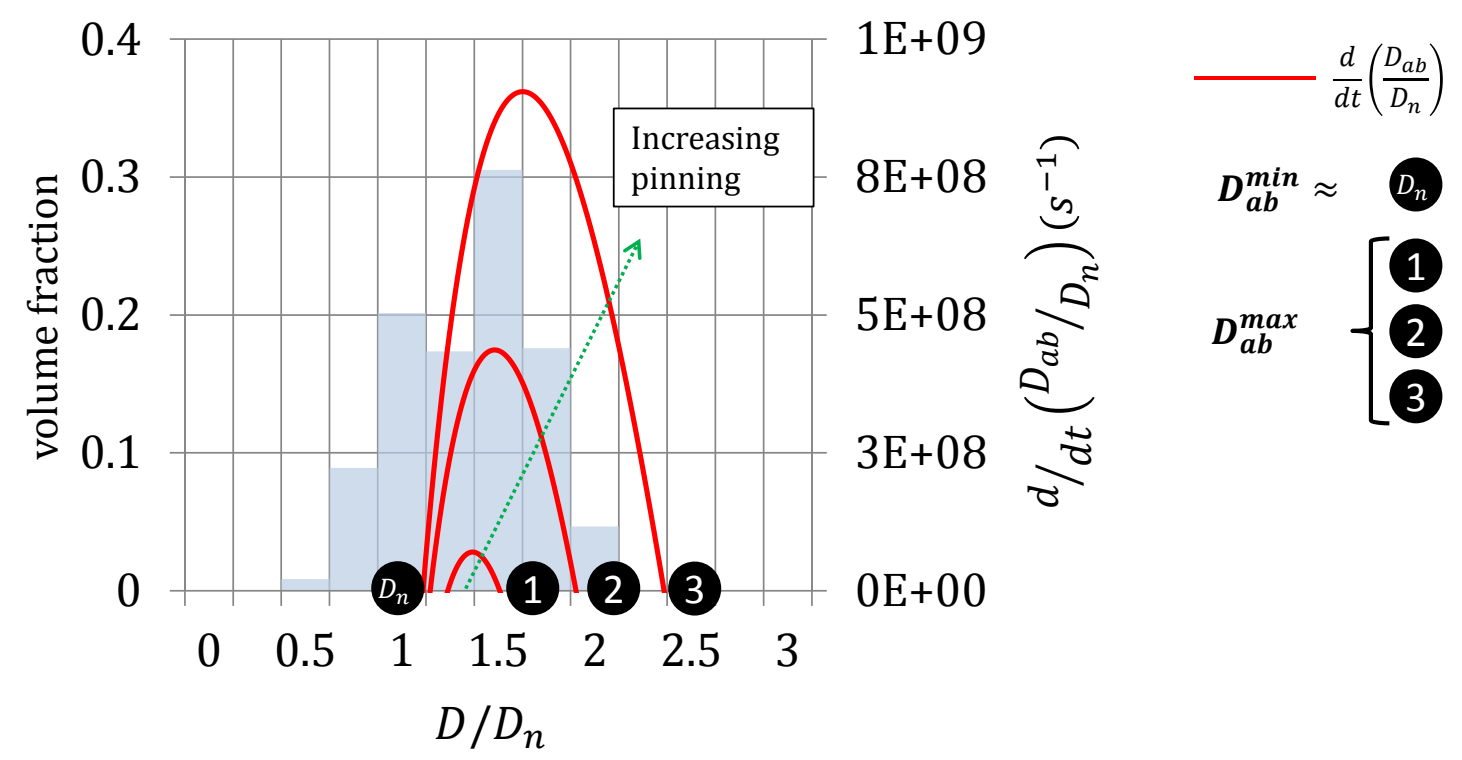

Figure 5.3: Superimposition AGG condition $\left(d / d t\left(D_{a b} / D_{n}\right)>0\right)$ on grain size distribution. $d / d t\left(D_{a b} / D_{n}\right)$ can range between $D_{n}$ and (1) or (2) or (3). Here, it is assumed that $M=1$.

which can grow comparatively faster than the mean size grains. In a zero pinning condition (no precipitate), for $\mu=0.56$ and $\lambda=0.6$, it can be found that AGG condition is valid between, $D_{a b}^{\min } / D_{n} \approx 1.26$ and $D_{a b}^{\max } / D_{n} \approx 1.43$.

In the figure $5.3, d / d t\left(D_{a b} / D_{n}\right)$ obtained for increasing pinning conditions are superimposed on a normal grain size distribution. As shown in the figure $5.3, D_{a b}^{\max }$ can be within the grain size distribution and very close to the mean sized grain (1) or close but smaller than the largest grain size (2) or larger than the maximum grain size in the distribution (3). Among these three situations only (3) bearing the probability of AGG initiation. In the (3), the largest grains in the microstructure can grow relatively faster than the mean. In the case of, (1) and (2) grain sizes close to the mean sized one grow faster which facilitate a quasi-stationary grain size distribution evolution (normal grain growth). From figure 5.3, it can be concluded that $D_{a b}^{\max }$ must be at least 2 times of $D_{n}$ in order for AGG to occur. So, a realistic qualitative identifier of AGG and NGG can be expressed as follows:

$$
\begin{aligned}
& D_{a b}^{\max } / D_{n}>2 \text { Probable grain growth condition: AGG } \\
& D_{a b}^{\max } / D_{n}<2 \text { Probable grain growth condition: NGG }
\end{aligned}
$$

In a static system with stationary mean grain $\left(D_{n}\right)$ size and pinning condition, it is sufficient to present $d / d t\left(D_{a b} / D_{n}\right)$ as a prediction of AGG. On the other hand, system with evolving mean grain size and pinning conditions, $D_{a b}^{\max } / D_{n}$ provides a much simpler representation. In order to present the evolution of AGG condition with time, $D_{a b}^{\max } / D_{n}$ is presented as the parameter showing AGG probability in 
the studied system.

\subsection{Implications of dynamic coupled model}

In this section different physical parameters controlling the grain growth conditions are discussed. Considering the mean austenite grain growth and evolution of precipitation conditions, implication of dynamic coupled modeling can be divided into the following categories:

1. Abnormal grain growth in presence of stable precipitation state.

2. Abnormal grain growth in presence of precipitate coarsening.

3. Abnormal grain growth in presence of precipitate nucleation.

4. Abnormal grain growth in presence on dissolving precipitate.

In the light of above mentioned conditions, dynamic coupled model behavior presented with different precipitation state is discussed. Physical properties like austenite mean grain size, precipitate volume fraction, mean radius and number density are varied to realize model sensitivity to predict AGG. Efforts are given to present a comparative picture of physical parameters and model predictions relationship.

\subsubsection{Abnormal grain growth in presence of stable precipi- tation state}

In figure 5.4 (a) and (b), volume fraction and the mean radius evolution of a stable precipitate is presented. Both the volume fraction and mean radius are constant with time thus a static precipitation state. The mean grain diameter $\left(D_{n}\right)$ is shown in figure 5.4 (c) which is evolving with time. It can be seen that all the studied grain sizes reached quite similar value at $10^{6} \mathrm{sec}$ in to the simulation.

Figure $5.4(\mathrm{~d})$ represents the ratio between maximum grain size $\left(D_{a b}^{\max }\right)$ with probability to grow abnormally $\left(d / d t\left(D_{a b} / D_{n}\right)>0\right)$ and the mean grain size $\left(D_{n}\right)$ evolution with time. In the stable precipitation state, initially grain sizes $3-7 \mu \mathrm{m}$ are in the AGG risk region as $D_{a b}^{\max } / D_{n}>2$ but $1 \mu \mathrm{m}$ remains below as $D_{a b}^{\max } / D_{n}<2$. The reason can be explained from the mean grain growth rate inversely proportional relationship with mean grain size. Smallest grain size's growth rate is comparatively higher and for this reason, the larger grain in this case can not grow comparatively faster $\left(d / d t\left(D_{a b} / D_{n}\right)<0\right)$ to induce AGG. It is already shown in chapter 2 (section 2.8), that in a constant precipitation state, increase in mean grain size increases the maximum grain size $\left(D_{a b}^{\max }\right)$ susceptible to AGG. Quite similar phenomenon can be observed in figure 5.4 (d). System with $1 \mu \mathrm{m}$ grain 


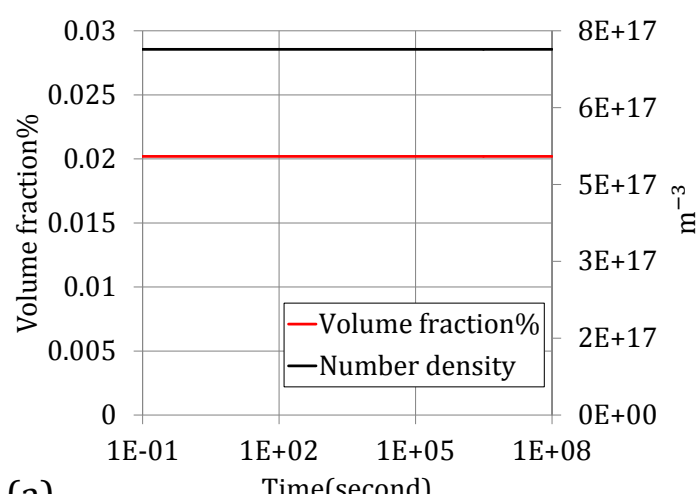

(a)

Time(second)

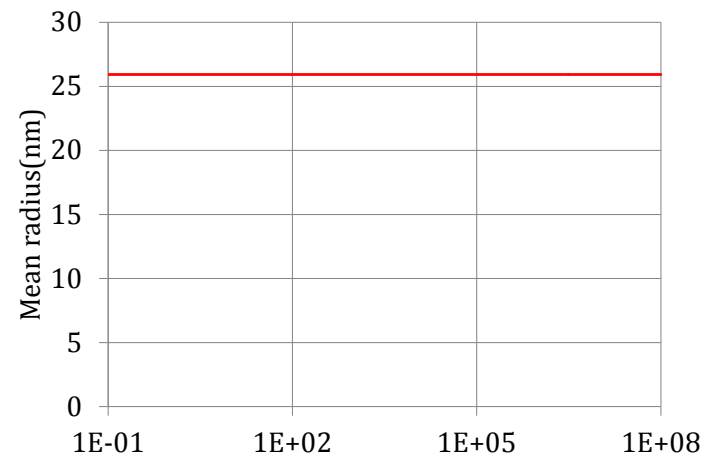

(b)
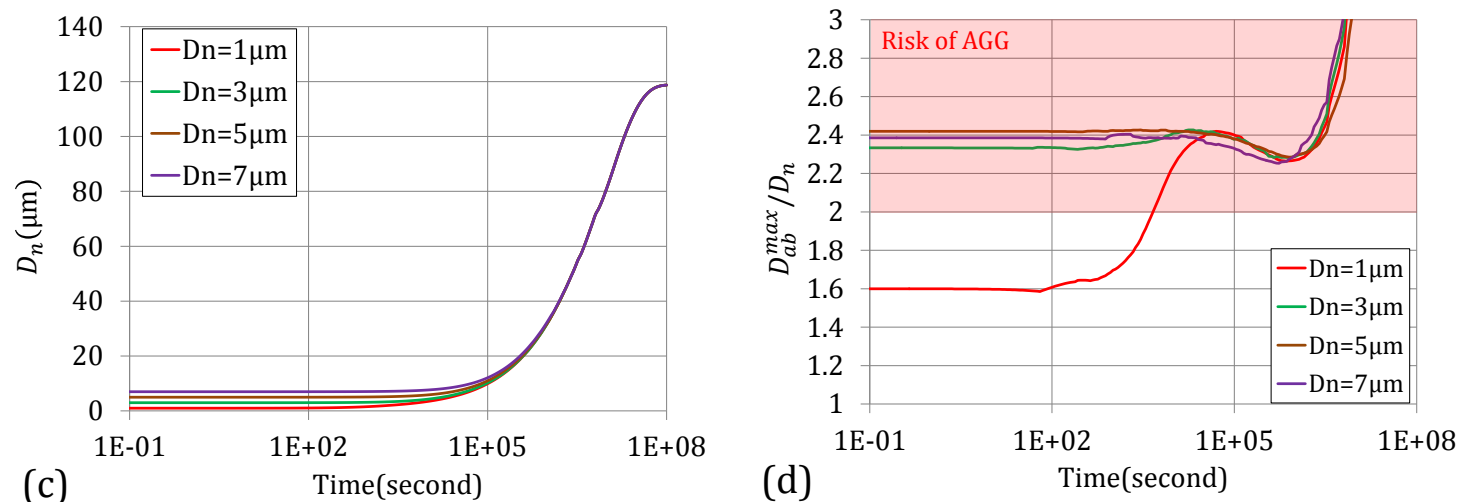

Figure 5.4: (a) Volume fraction and number density $\left(\mathrm{m}^{-3}\right)$ evolution of a stable precipitate, (b) precipitate mean radius evolution, (c) mean austenite grain size $\left(D_{n}\right)$ evolution and (d) abnormal grain growth condition $\left(D_{a b}^{\max } / D_{n}\right.$ where, $\left.d / d t\left(D_{a b} / D_{n}\right)>0\right)$ evolution for different initial austenite grain size. Here, temperature $T=900^{\circ} \mathrm{C}$ and the AGG model parameter $\lambda=0.6$ and $\mu=0.56$. 

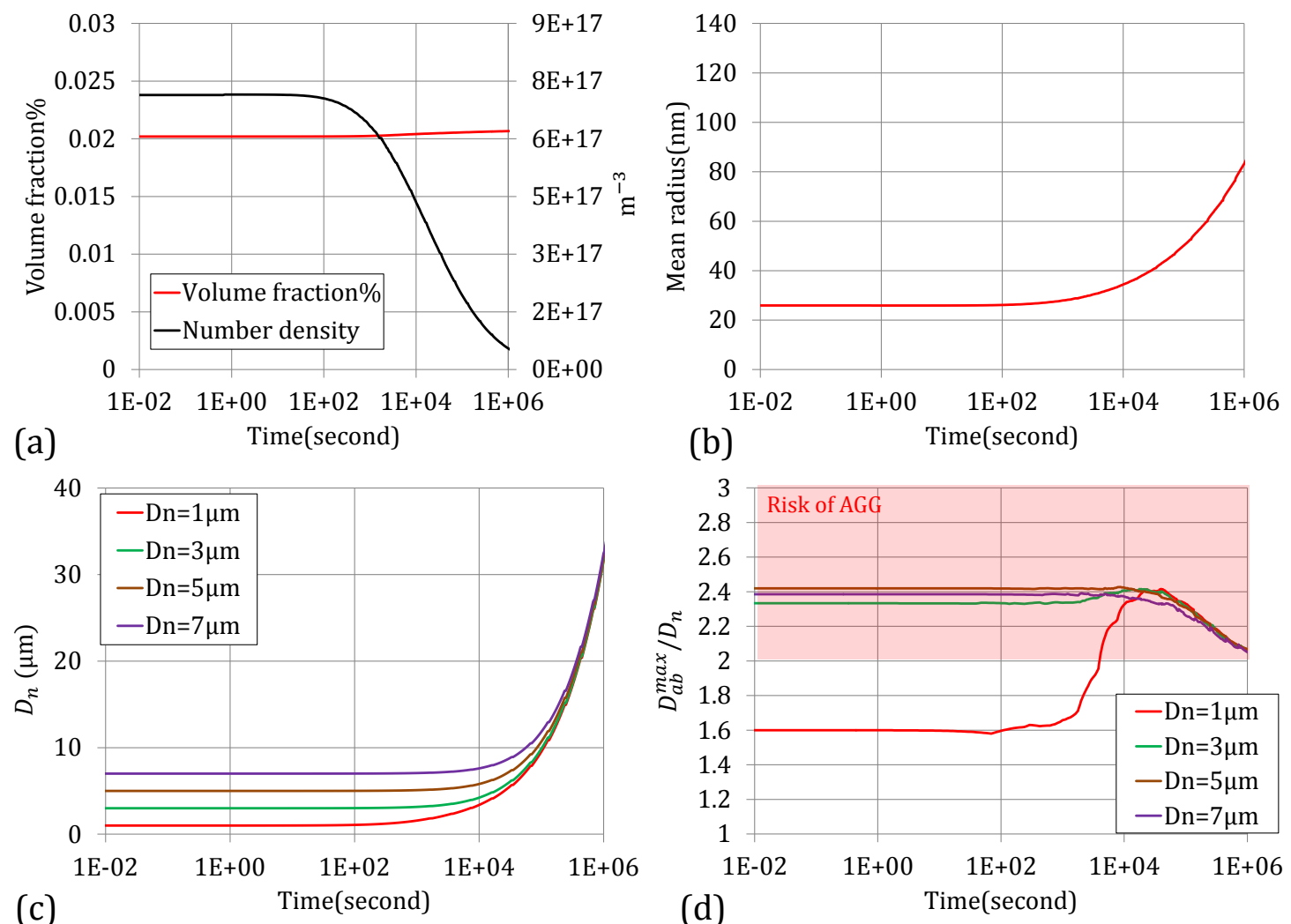

Figure 5.5: (a) Volume fraction and number density $\left(\mathrm{m}^{-3}\right)$ evolution of a precipitate, (b) precipitate mean radius evolution, (c) mean grain size evolution and (d) maximum grain size $\left(D_{a b}^{\max }\right)$ relative to the mean grain size $\left(D_{n}\right)$ which has the probability to grow abnormally. Here, temperature $T=900^{\circ} \mathrm{C}$, the model parameters $\mu=0.56$ and $\lambda=0.6$.

size enters in the AGG risk zone at $10^{4} \mathrm{sec}$ when the grain size increased to $5 \mu \mathrm{m}$. Systems with the starting mean grain sizes between 3-7 $\mu \mathrm{m}$ rather show an accelerated increase in $D_{a b}^{\max }$ just above $10^{6} \mathrm{sec}$ in the simulation. As stated earlier, an increase in mean grain size decreases the mean grain growth rate. Consequently grains with size advantage $\left(D_{a b}\right)$ in the microstructure can out grow (in terms of growth rate) the smaller ones $\left(D_{n}\right)$. This results is an increase AGG susceptibility in the microstructure.

\subsubsection{Abnormal grain growth in presence of precipitate coars- ening}

In figure 5.5 (a) volume fraction and number density evolution is presented and the mean radius evolution of a coarsening precipitation state is shown in figure 5.5 (b). Here, the precipitate volume fraction (see figure 5.5(a)) remains constant but the number density decreases due to coarsening. Precipitate coarsening can be 
observed by the increase in mean radius (see figure $5.5(\mathrm{~b})$ ).

Figure 5.5 (d) represents the ratio between maximum grain size $\left(D_{a b}^{\max }\right)$ with the probability to grow abnormally $\left(d / d t\left(D_{a b} / D_{n}\right)>0\right)$ and the mean grain size $\left(D_{n}\right)$. In this precipitate coarsening condition, the results suggest that initially there is no possibility of abnormal grain growth for the mean grain size of $1 \mu \mathrm{m}$ $\left(D_{a b}^{\max } / D_{n}<2\right)$. At the beginning of the simulation, $1 \mu \mathrm{m}$ mean grain size has higher growth pressure $\left(d D_{n} / d t\right)$ than 3,5 and $7 \mu \mathrm{m}$. The pre-existing larger grains (at least 2 times larger than $D_{n}$ ) in the microstructure can not grow comparatively faster thus no probability of AGG. An increase in holding time allows the mean grain size to evolve. In fact, just above $10^{4} \mathrm{sec} 1 \mu \mathrm{m}$ mean grain size coarsen to $\sim 3 \mu \mathrm{m}$ and enters the AGG risk region. A decrease in precipitate number density also contributes to the increase in larger grains $\left(D_{a b}\right)$ relative growth rate $\left(d / d t\left(D_{a b} / D_{n}\right)\right)$ thus AGG risk. In comparison with the soft coupled (SCM) approach (see chapter 4 section 4.3.1), significant differences can be observed for $1 \mu \mathrm{m}$ mean grain size system. The SCM approach suggest that $1 \mu \mathrm{m}$ system never enters the AGG risk region while the DCM approach shows that AGG is probable in the intermediate time $\left(\sim 10^{4}-10^{6} \mathrm{sec}\right)$. DCM allows the mean grain size to evolve and the system returns to the NGG region at higher holding time ( $>10^{6} \mathrm{sec}$ ). The AGG to NGG transformation is due to the decrease in pinning in the system. A low pinning condition allows an increase in mean grain growth rate and consequently decreases the relative growth rate $\left(d / d t\left(D_{a b} / D_{n}\right)\right)$ of the larger grains. As the larger grains in the system can not growth comparatively faster, the grain growth condition migrate to the NGG region.

Figure 5.5 (d) suggest that for the initial mean grain size $D_{n}=3$ to $5 \mu \mathrm{m}$, from the very beginning of simulation there is probability of AGG $\left(D_{a b}^{\max } / D_{n}>2\right)$. It can be seen that with the precipitate coarsening (increase in mean radius) and the initial mean grain size evolution (increase in mean grain size) there is a decrease in the maximum grain size that lies in the risk of AGG. It is understandable that a decrease in precipitate number density decreases the total pinning force. This results in an increase in mean grain growth rate. As stated earlier, higher mean grain growth rate decreases the AGG risk. In comparison with the SCM (see chapter 4 section 4.3.1), the DCM approach yield quite different evolution of AGG condition for $D_{n}=3$ to $5 \mu \mathrm{m}$. In the SCM approach, a sequential AGG to NGG transformation can be observed. The $3 \mu \mathrm{m}$ initial mean grain size system enters the NGG region earlier than 5 and $7 \mu \mathrm{m}$. The initial mean grain size evolved to quite similar size in DCM approach and as a results a concurrent transformation from AGG to NGG can be observed. From kinetic point of view, this observation refers to the equilibrium between the mean grain growth rate and available pinning in the microstructure. A dynamic shift of this equilibrium (in DCM) to a similar 

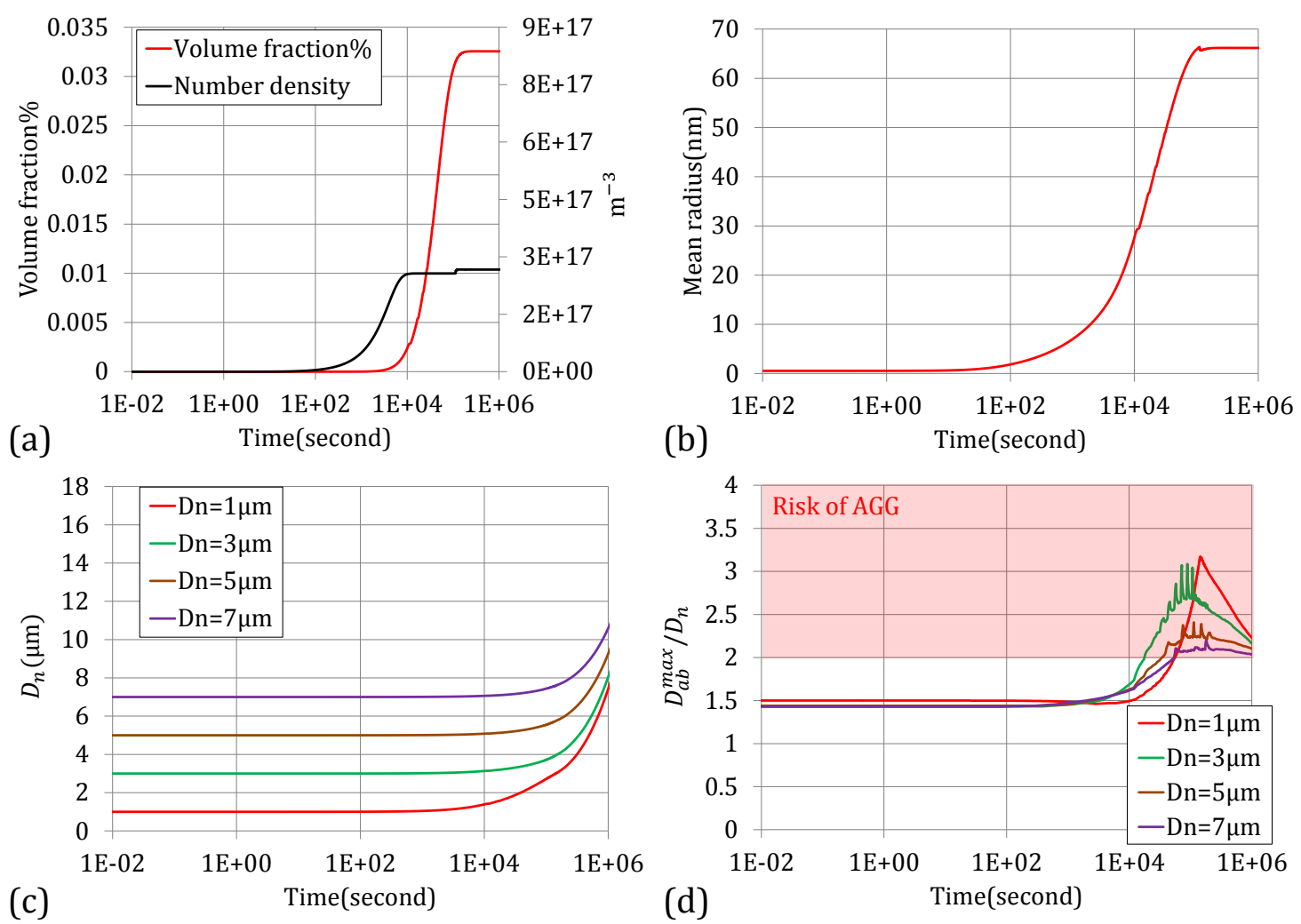

Figure 5.6: (a) Volume fraction and number density $\left(\mathrm{m}^{-3}\right)$ evolution of a nucleating precipitate, (b) precipitate mean radius evolution, (c) Mean austenite grain size $\left(D_{n}\right)$ evolution with time and (d) maximum grain size $\left(D_{a b}^{\max }\right)$ relative to the mean grain size $\left(D_{n}\right)$ which has the probability to grow abnormally. Here, temperature $T=800^{\circ} \mathrm{C}$, the AGG model parameter $\lambda=0.6$ and $\mu=0.56$.

state allows all the studied initial mean grain size system to experience AGG to NGG transformation at the same time. As the SCM approach does not consider the mean grain size evolution, this equilibrium is shifted only by the change in pinning (precipitation state). It can be concluded that in a pre-existing AGG condition, AGG probability decreases with precipitate coarsening (decreasing number density) and it becomes faster with mean grain size evolution.

\subsubsection{Abnormal grain growth in presence of precipitate nu- cleation}

In figure 5.6 (a) volume fraction and number density evolution are presented and the mean radius evolution of a coarsening precipitation state are shown in figure 5.6 (b). Here the precipitate volume fraction and number density (see figure 5.6(a)) starting from zero increases with time. Simultaneously precipitate growth occurs which can be observed by the increase in mean radius (see figure 5.6(b)). The mean grain size evolution is shown in the figure $5.6(\mathrm{~d})$. 
Figure 5.6 (d) represents the ratio between maximum grain size $\left(D_{a b}^{\max }\right)$ with probability to grow abnormally $\left(d / d t\left(D_{a b} / D_{n}\right)>0\right)$ and the mean grain size $\left(D_{n}\right)$. It can be observed from figure $5.6(\mathrm{~d})$, that at the beginning there is no probability of AGG for all the mean grain sizes as $D_{a b}^{\max } / D_{n}<2$. In fact, $D_{a b}^{\max } / D_{n} \approx 1.4$ and no probability of AGG can be observed up to time less than $10^{4} \mathrm{sec}$. As the grain size with the probability to grow comparatively faster is very close to the mean grain size $\left(D_{n}\right)$, the microstructure should remain quasi-stationary (NGG). Figure 5.6 (c) suggest that up to $10^{4} \mathrm{sec}$, the mean sizes also remain quite unchanged. $D_{a b}^{\max } / D_{n}$ shows an increase above $10^{4} \mathrm{sec}$ in the simulation, as the precipitate volume fraction reaches $0.005 \%$. In the mean time, precipitate number density reaches the maximum quantity $\left(\sim 2.9 \times 10^{17} \mathrm{~m}^{-3}\right)$ and mean radius $\sim 30 \mathrm{~nm}$. The precipitate size distribution at this period is in such a state that the induced pinning is sufficient to slow the mean grain growth rate. As a result, the larger grains $\left(D_{a b}\right)$ in the microstructure can grow relatively faster $\left(d / d t\left(D_{a b} / D_{n}\right)\right)$ than the smaller grains $\left(D_{n}\right)$ and AGG probability increases.

From figure 5.6 (a) and figure 5.6 (d), it is evident that abnormal grain growth probability manifest when there is a certain volume fraction and number density of precipitates. As the precipitation state enters the coarsening regime, maximum grain size with potential to grow abnormally decreases. This microstructural behavior of transformation from NGG to AGG and then again to NGG can be linked with two interconnected factors: (1) mean austenite grain size $\left(D_{n}\right)$ and (2) pinning by precipitates. As shown in the previous section 5.3.1, in an approximately constant pinning condition, increase in mean austenite grain size decreases AGG probability (decrease in total interfacial energy thus decreasing AGG driving force). Similarly, with coarsening, the precipitate number density decreases and lowers the probability of AGG. A microstructure experiencing AGG at some moment into the heat treatment, facilitated by high grain boundary mobility, has higher certainty to attain AGG microstructure. Even if the conditions transform to NGG, it is more likely that it would require longer holding time to transform the AGG microstructure to NGG.

DCM depicts a realistic scenario of grain growth condition in presence of precipitate nucleation. The grain growth condition transform shows NGG $\rightarrow$ AGG $\rightarrow$ NGG. The Soft Coupled Modeling (SCM) (see chapter 4 section 4.3.2) shows NGG to AGG transformation for 3-7 $\mu \mathrm{m}$ mean grain size on the same time scale of $10^{6} \mathrm{sec}$. So, in DCM approach the kinetics of grain growth condition transformation is faster than the one obtained by SCM. 

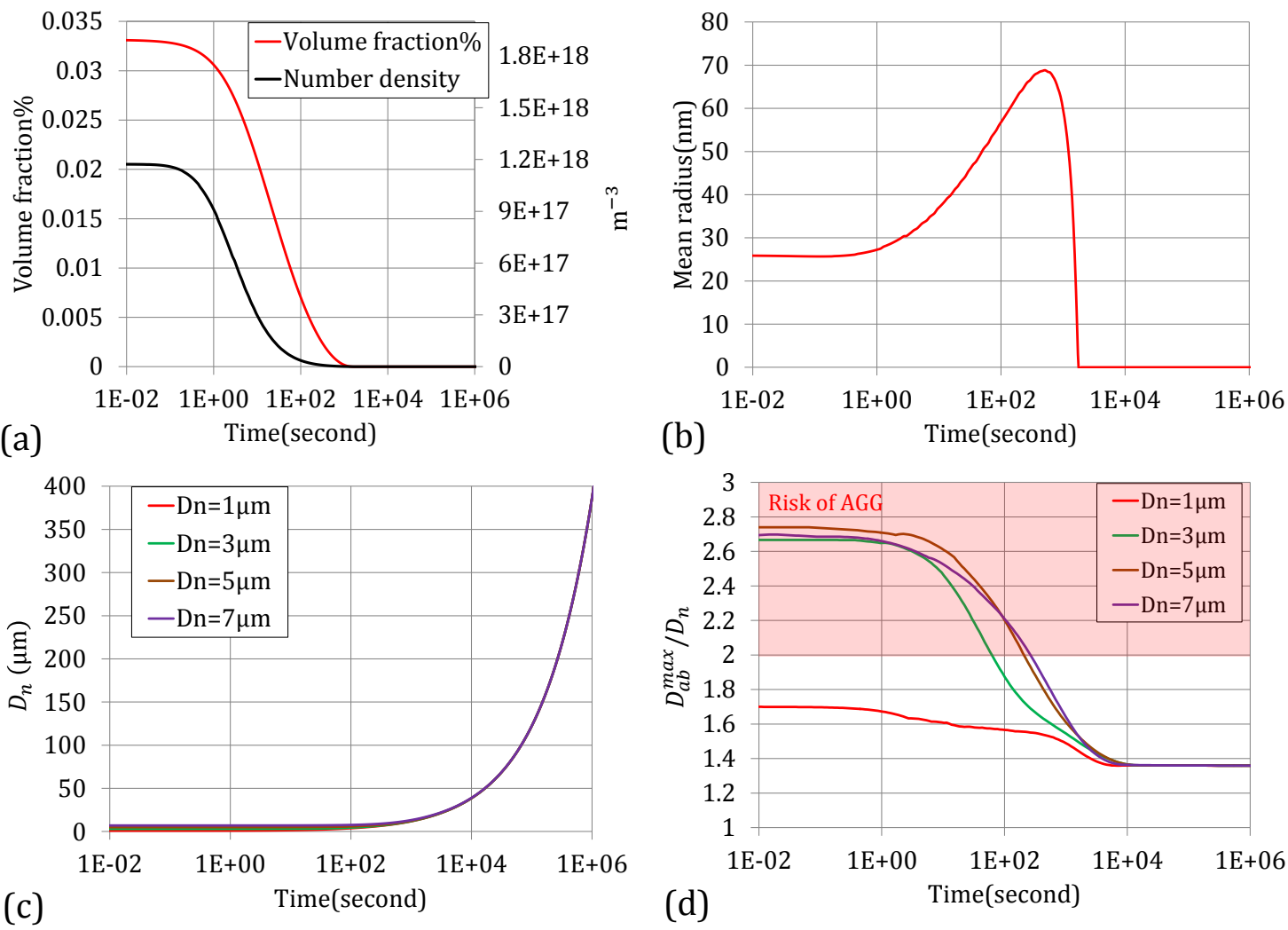

Figure 5.7: (a) Volume fraction and number density $\left(\mathrm{m}^{-3}\right)$ evolution of a dissolving precipitate, (b) precipitate mean radius evolution, (c) mean austenite grain size $\left(D_{n}\right)$ evolution and (d) maximum grain size $\left(D_{a b}^{\max }\right)$ relative to the mean grain size $\left(D_{n}\right)$ which has the probability to grow abnormally. Here, temperature $T=$ $1100^{\circ} \mathrm{C}$, the AGG model parameter $\lambda=0.6$ and $\mu=0.56$.

\subsubsection{Abnormal grain growth in presence on dissolving pre- cipitate}

In figure 5.7 (a), evolution of a dissolving precipitate volume fraction and number density is presented. Precipitate mean radius evolution is shown in figure 5.7 (b). Precipitate volume fraction and number density decreases with time and finally completely dissolves. Initially, an increase in precipitate mean radius can be observed (see figure 5.7(b)) as the smaller precipitate classes in the distribution dissolve faster than the large ones. When there are only larger precipitate classes remain, the mean radius decreases rather quickly.

In figure $5.7(\mathrm{~d})$, the ratio between maximum grain size $\left(D_{a b}^{\max }\right)$ with probability to grow abnormally $\left(d / d t\left(D_{a b} / D_{n}\right)>0\right)$ and the mean grain size $\left(D_{n}\right)$ is presented. Figure 5.7 (c) suggest an rapid growth of all the studied mean grain sizes. In absence of precipitate induced pinning, the austenite grains can growth freely and initial mean grain size $1-7 \mu \mathrm{m}$ reached $\sim 400 \mu \mathrm{m}$ at $10^{6}$ sec.

It can be observed from figure 5.7(d) that all the investigated mean grain sizes 
Table 5.1: Composition of industrial alloy steel-A in atomic \%

\begin{tabular}{cccccccccc}
\hline $\mathrm{C}$ & $\mathrm{Si}$ & $\mathrm{Mn}$ & $\mathrm{Ni}$ & $\mathrm{Cr}$ & $\mathrm{V}$ & $\mathrm{Ti}$ & $\mathrm{Al}$ & $\mathrm{Nb}$ & $\mathrm{N}$ \\
0.702 & 0.407 & 0.455 & 1.22 & 1.47 & 0.00696 & 0.00194 & 0.0549 & 0.00171 & 0.0598 \\
\hline
\end{tabular}

$\left(D_{n}\right)$ except $D_{n}=1 \mu \mathrm{m}$, has the potential to experience AGG $\left(D_{a b}^{\max } / D_{n}>2\right)$. $D_{a b}^{\max } / D_{n}$ shows a gradual decrease with decreasing precipitate volume fraction and finally reaches $\sim 1.4$ at $10^{4}$ sec. The probability of AGG occurrence indeed diminishes just above $10^{2} \mathrm{sec}$ in to the simulation as $D_{a b}^{\max } / D_{n}$ reaches below 2 . It can be stated that probability of AGG occurrence decreases with decreasing precipitate volume fraction and number density. In absence of precipitate there is no probability of AGG in the microstructure. Comparing the SCM (see chapter 4 section 4.3.3) and DCM outcome, an exactly similar trend in grain growth condition evolution can be observed. This comparison points out that during dissolution the grain growth condition evolution is solely controlled by the precipitation state. The mean grain size has small effects in controlling the grain growth condition.

\subsection{Application to low alloy steel}

In this section, application of the dynamic coupled model (DCM) in two industrial alloys: (1) steel-A and (2) steel-B is discussed. DCM predictions are validated by comparing with the experimental results. Modeling results agreements and disagreements are discussed in order to clarify the capabilities and limitations of the dynamic coupled approach.

In the following discussion, both alloys are presented in detail. The compositions and heat treatment cycles utilized are also discussed. Experimentally measured precipitation state and austenite grain size distributions of different heat treated conditions of the two alloys are also presented. A comparative picture between the different states of alloys are done to understand the relation between precipitation state and Abnormal Grain Growth (AGG). Finally, the DCM is applied to the two alloys and a time-temperature mechanism map is build to depict a detailed relationship between heat treatment temperature and grain growth conditions. 


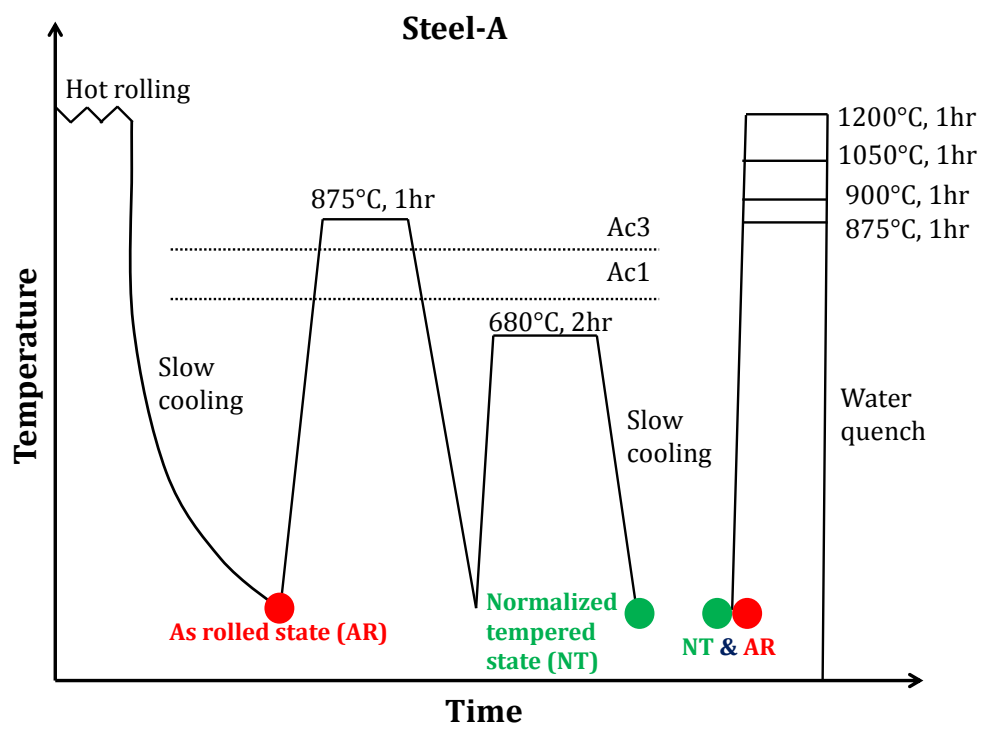

Figure 5.8: Heat treatment cycle of alloy steel-A to obtain As Rolled (AR) and Normalized Tempered (NT) state.

\subsubsection{Industrial alloy: steel-A}

\subsubsection{Composition and heat treatment}

Composition of the industrial alloy steel-A is given in the table 5.1. The main precipitating element in the alloy is Al. Energy Dispersive X-Ray (EDX) analysis of precipitate on carbon extraction replica in Transmission Electron Microscope (TEM) confirmed the precipitate's chemistry (details are given in appendix A). Besides that precipitates analysis using Induction Coupled Plasma (ICP) analyzer (see appendix A) also showed that only AlN quantity is high enough to impact the grain growth condition.

The heat treatment carried out on steel-A is shown in figure 5.8. The As Rolled (AR) state is obtained by hot rolling of the as cast section of the alloy. The AR state is then heat treated $\left(>A C_{3}\right)$ at $875^{\circ} \mathrm{C}$ for $1 \mathrm{hr}$ and $\left(<A C_{1}\right)$ at $680^{\circ} \mathrm{C}$ for $2 \mathrm{hr}$ followed by air cooling to produce the Normalized Tempered (NT) state. Metallographic analysis suggested that the initial microstructure of the alloy is bainitic.

Austenitizing treatment is performed on both AR and NT states at $875^{\circ} \mathrm{C}$, $900^{\circ} \mathrm{C}, 1050^{\circ} \mathrm{C}$ and $1200^{\circ} \mathrm{C}$ for different holding time. The samples are quenched in order to retain austenite grain boundaries impression in the microstructure. The quenched sample's prior austenite grain boundaries are revealed by the hot picric acid solution modified with a surface reactant (see appendix A for details). The precipitate volume fraction in a heat treated state is measured by dissolving the matrix using a potentiostat in a solution of salicylic acid, lithium chloride and 
methanol. The filtered precipitates dissolved in a highly acidic solution $(H C L+$ $\mathrm{H}_{2} \mathrm{SO}_{4}+\mathrm{HF}+\mathrm{HNO}_{3}$ ) are then analyzed in the ICP to measure the precipitate quantity (see appendix A for details). Precipitate volume fraction is measured in terms of substitutional element's mass fraction in PPM. The PPM can be converted to the precipitate volume fraction using the expression 5.5.

$$
f_{v}^{A_{x} B_{y}}(T, t)=f_{m}^{A} \frac{\rho_{F e}}{\rho_{A_{x} B_{y}}}\left(\frac{y}{x} \frac{M_{B}}{M_{A}}+1\right)
$$

Here, $f_{v}^{A_{x} B_{y}}(T, t)$ is the volume fraction of $A_{x} B_{y}$ precipitate, $f_{m}^{A}$ is the mass fraction of element $A$ measured using ICP, $\rho_{F e}$ and $\rho_{A_{x} B_{y}}$ are the molecular density of the matrix and the precipitate and $M_{A}$ and $M_{B}$ are the molar mass of the substitutional and interstitial elements.

Precipitate size distribution is measure by analyzing carbon extraction replica in TEM using the High Angle Annular Dark Field (HAADF) mode. Images taken using the HAADF mode are analyzed by image processing software to obtain the equivalent circle radius of the precipitates. The measured precipitate radiuses are used to build the size distribution (see appendix A). Normalizing the size distribution with the volume fraction give us the number density (Number of precipitate $/ \mathrm{m}^{-3}$ ) of the precipitate classes. In the present study, precipitate size distribution is presented as $\mathrm{m}^{-4}$ where the number density per class $\left(\mathrm{m}^{-3}\right)$ is divided by the class width $(\Delta R)$.

\subsubsection{Precipitation state and grain size evolution}

A detailed discussion on the precipitation states and grain size distributions of the AR and NT states heat treated at $875^{\circ} \mathrm{C}, 900^{\circ} \mathrm{C}, 1050^{\circ} \mathrm{C}$ and $1200^{\circ} \mathrm{C}$ for different holding time is presented in chapter 4 section 4.4.1.2. In the following section, effects of heating rates on grain growth condition are discussed.

\subsubsection{Effects of heating rates on grain growth}

In the following discussion, effects of heating rate on austenite grain growth behavior is presented. Steel-A's As Rolled (AR) and Normalized Tempered (NT) state is heated to $1050^{\circ} \mathrm{C}$ at the rate of $0.1,0.5$ and $1^{\circ} \mathrm{C} / \mathrm{min}$ and then directly quenched in water. In order to simulate different heating rates, "Gleeble" thermomechanical simulator is used. In the "Gleeble", precise control over heating rate is possible but the uniformly heated zone in the specimen is confined to a very small region ( $\sim 5 \mathrm{~mm}$ in length) in the middle along the direction of the two grips. For this reason, after the heat treatment, only the center cross section of the specimens are analyzed to determine the prior austenite grain size. 

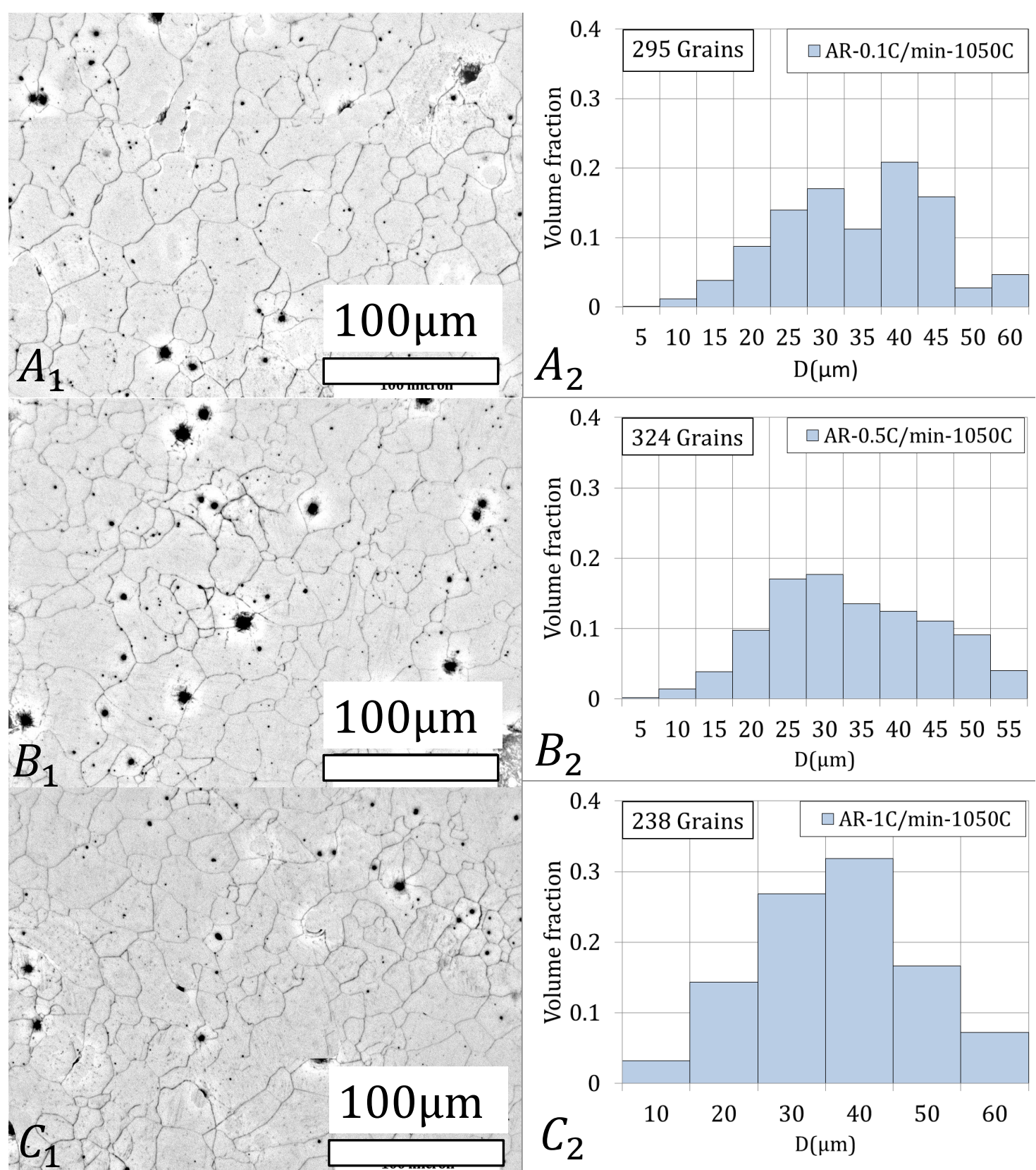

Figure 5.9: Grain size distribution in industrial alloy steel-A's As Rolled (AR) state heated to $1050^{\circ} \mathrm{C}$ and then water quenched. $A_{2}: 0.1^{\circ} \mathrm{C} / \mathrm{min}$ and $A_{1}$ : Corresponding micrograph, $B_{2}: 0.5^{\circ} \mathrm{C} / \mathrm{min}$ and $B_{1}$ : corresponding micrograph and $C_{2}: 1^{\circ} \mathrm{C} / \mathrm{min}$ and $C_{1}$ : Corresponding micrograph. 
In figure 5.9, prior austenite grain size distributions of the AR state subjected to $0.1,0.5$ and $1^{\circ} \mathrm{C} / \mathrm{min}$ heating rates are presented. From figure 5.9 , it can be seen that the grain size distribution remained normal (NGG), which can be confirmed by the adjacent micrographs. This suggests that for all the heating rates, AR state shows a normal grain growth microstructure. This observation is quite similar to the case where AR state is heat treated at $1050^{\circ} \mathrm{C}$ for different times (see figure 4.11).

Prior austenite grain size distribution of the NT state subjected to $0.1^{\circ} \mathrm{C} / \mathrm{min}$, $0.5^{\circ} \mathrm{C} / \mathrm{min}$ and $1^{\circ} \mathrm{C} / \mathrm{min}$ heating rates are presented in figure 5.10. It can be observed that except for $1^{\circ} \mathrm{C} / \mathrm{min}$ heating rate, all the other heating rates show presence of AGG. As can be seen from figure $5.10\left(A_{2}\right)$ and $\left(B_{2}\right)$, the largest grains in the distribution occupying higher or equal volume fraction in comparison with the smaller ones. On the other hand, the highest heating rate of $1^{\circ} \mathrm{C} / \mathrm{min}$, showing a normal microstructure (see figure $5.10\left(C_{1}\right)$ and $\left(C_{2}\right)$ ). A comparison between AR and NT states is presented in figure 5.11, in terms of cumulative volume fraction of largest $10 \%$ by number grains present in the grain size distribution. It can be seen from figure 5.11, that in the NT state heat treated at 0.1 and $0.5^{\circ} \mathrm{C} / \mathrm{min}$ has $\sim 70$ $75 \%$ of the total volume occupied by the largest grains in the microstructure while the $1^{\circ} \mathrm{C} / \mathrm{min}$ has $\sim 50 \%$. This $\sim 20 \%$ difference in volume occupied by the largest grains is an indication that there is an initiation of $\mathrm{AGG}$ in 0.1 and $0.5^{\circ} \mathrm{C} / \mathrm{min}$ heat treated states. In the $\mathrm{AR}$ state heat treated at $0.1,0.5$ and $1^{\circ} \mathrm{C} / \mathrm{min}$ has $\sim 45$ to $55 \%$ of the total volume occupied by the largest $10 \%$ grains which is again a confirmation of normal grain growth.

In the literature $[4,65]$, it is frequently reported that AGG in prior austenite grain increases with increasing heating rates. On the contrary, results obtained from the NT state shows NGG microstructure for higher heating rate $\left(1^{\circ} \mathrm{C} / \mathrm{min}\right)$ but AGG for the slower ones $\left(0.1\right.$ and $\left.0.5^{\circ} \mathrm{C} / \mathrm{min}\right)$. The reason can be correlated to the fact that for slower heating rates, prior austenite grains in the NT state experienced some degrees of holding near the potential AGG initiation temperature of $\sim 1050^{\circ} \mathrm{C}$ (isothermal holding already showed that). Even the short period of holding allowed AGG to propagate, thus in the final microstructure it is visible. The faster heating rate of $1^{\circ} \mathrm{C} / \mathrm{min}$, allowed a short period of holding near $\sim 1050^{\circ} \mathrm{C}$. Shorter holding time might not allowed AGG to propagate and as a result NGG microstructure is observed. Observations from different heating rates, allowed us to realize that AGG is not only depends on the heating rate but also the holding time at that particular temperature. Higher heating rates can facilitate the formation of finer (smaller) mean austenite grain size but in order for the AGG to propagate at any temperature, sufficient holding is required. 

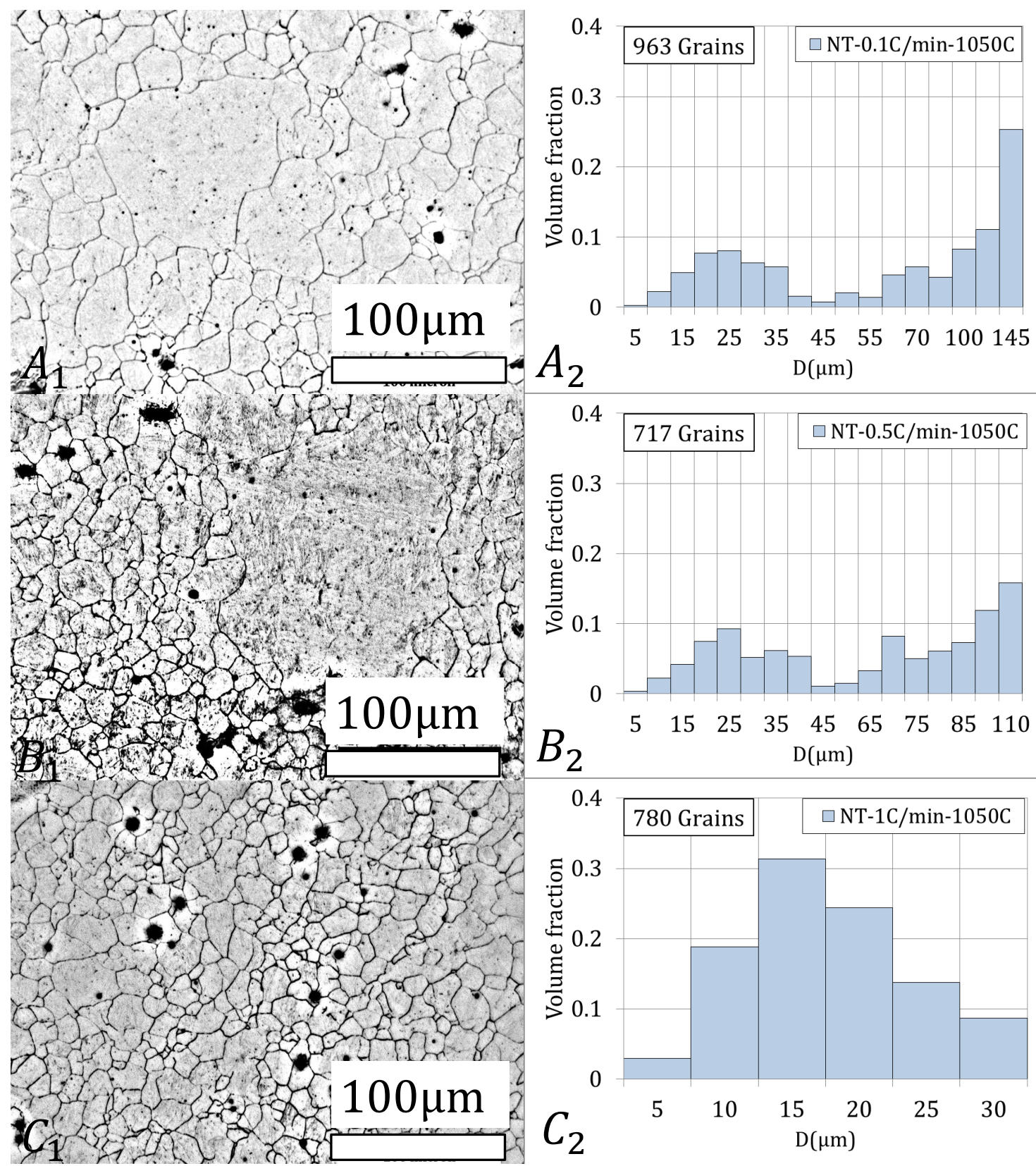

Figure 5.10: Grain size distribution in industrial alloy steel-A's Normalized Tempered (NT) state heated to $1050^{\circ} \mathrm{C}$ and then water quenched. $A_{2}: 0.1^{\circ} \mathrm{C} / \mathrm{min}$ and $A_{1}$ : Corresponding micrograph, $B_{2}: 0.5^{\circ} \mathrm{C} / \mathrm{min}$ and $B_{1}$ : corresponding micrograph and $C_{2}: 1^{\circ} \mathrm{C} / \mathrm{min}$ and $C_{1}$ : Corresponding micrograph. 


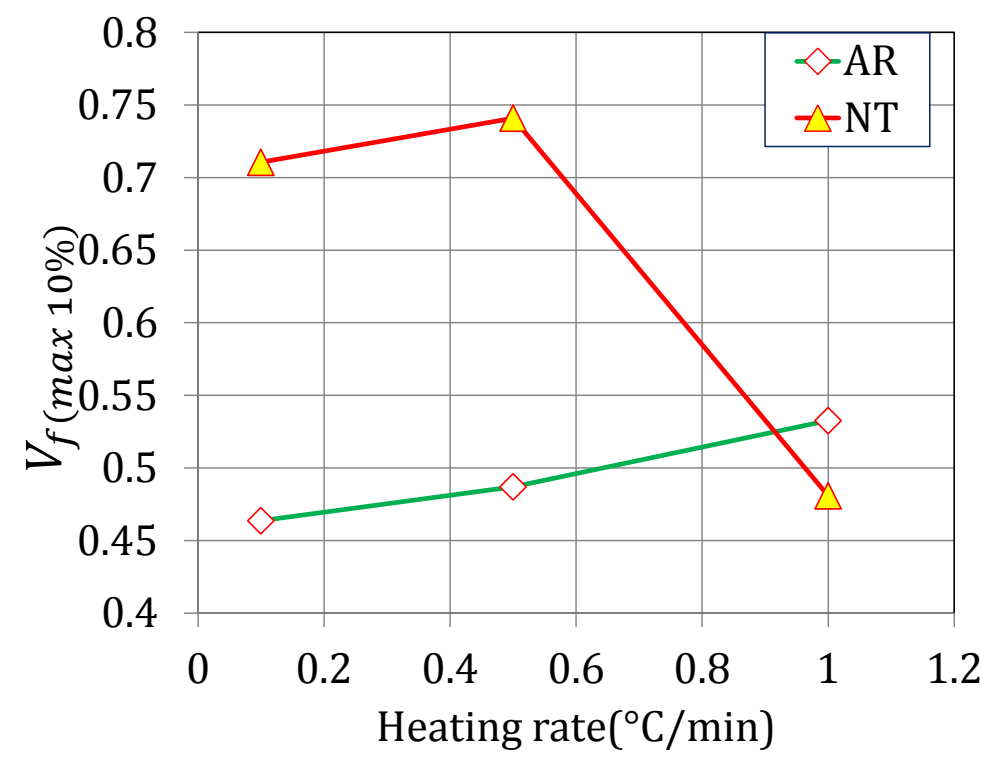

Figure 5.11: Cumulative volume fraction of largest $10 \%$ grains by number of the $\mathrm{AR}$ and NT states subjected to $0.1^{\circ} \mathrm{C} / \mathrm{min}, 0.5^{\circ} \mathrm{C} / \mathrm{min}$ and $1^{\circ} \mathrm{C} / \mathrm{min}$ till $1050^{\circ} \mathrm{C}$, followed by water quench.

\subsubsection{Model prediction}

Dynamic Coupled Modeling (DCM) is consists of two separate modeling schemes: (1) Precipitation model and (2) Abnormal Grain Growth (AGG) model. The modeling approach discussed here can simultaneously predict the precipitation state and mean grain size evolution. Capability to predict interconnected microstructural properties are the key strength of the DCM approach. Here the calculated precipitation state and mean grain size are considered to predict the probability of AGG and NGG in the microstructure. In the following discussion, model prediction in the case of isothermal heating and also for different heating rates are discussed in details.

\section{Grain growth condition in isothermal heat treatment}

It is essential to predict microstructural properties such as precipitate volume fraction, mean radius and mean grain size to calculate an accurate prediction about grain growth condition (AGG/NGG). In order to validate the coupled modeling, model prediction of precipitate volume fraction, mean radius and mean austenite grain size is presented in comparison with experimental data in figure 5.12. It can be seen from figure 5.12 (a), (b) and (c) that the model prediction is quite in agreement with the experimental observations.

Figure 5.13 represents the model prediction of grain growth conditions (AGG 

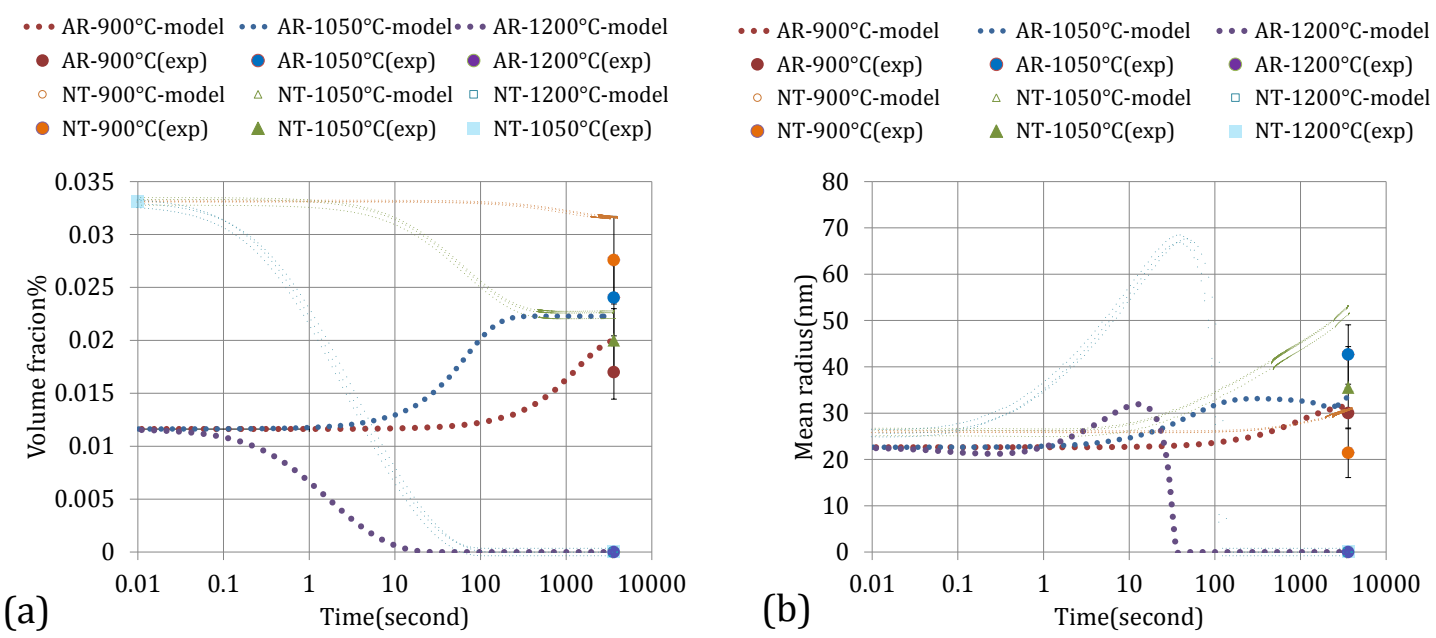

(a)
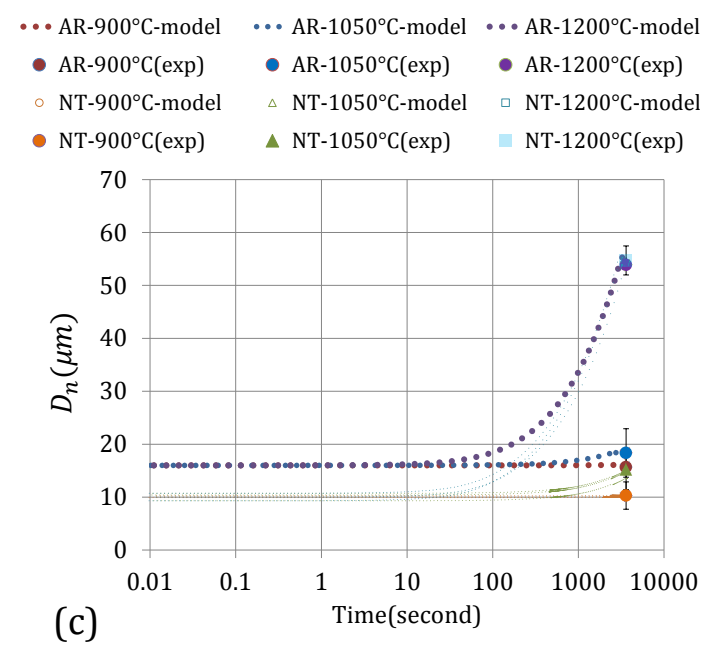

Figure 5.12: Steel-A: AR and NT state's (a) precipitate volume fraction, (b) precipitate mean radius and (c) mean grain size $\left(D_{n}\right)$ evolution at different temperatures are shown. 


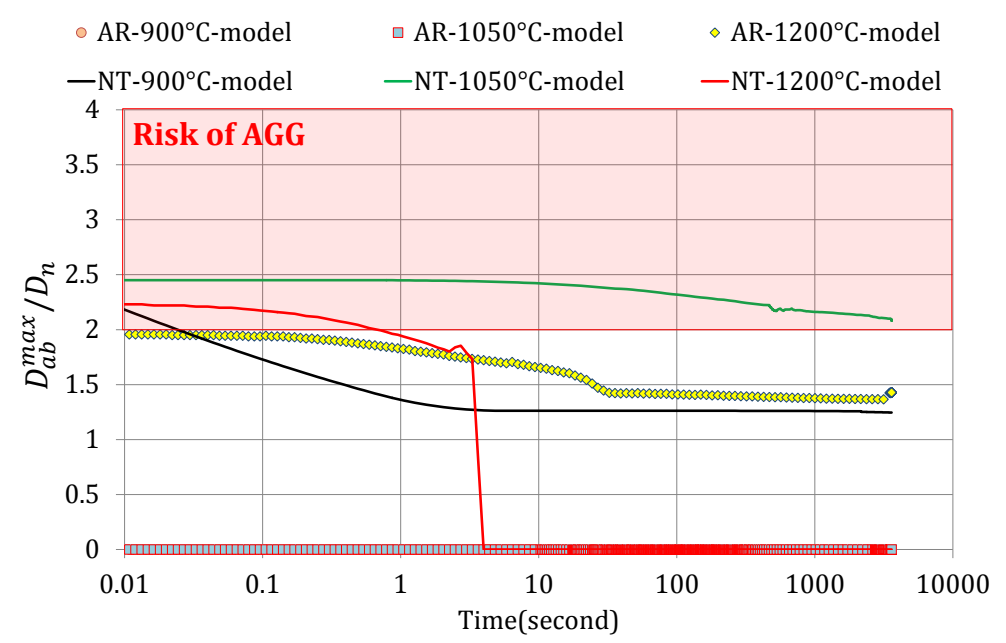

Figure 5.13: Dynamic coupled model prediction for industrial alloy steel-A's AR and NT state. The red shaded zone mark the risk of AGG area for $D_{a b}^{\max } / D_{n}$ value. Here, model parameters $\mu=0.54$ and $\lambda=0.6$.

or NGG) in the AR and NT states heat treated at different temperatures. It can be seen from figure 5.13 that in the $\mathrm{AR}$ state heat treated at $900^{\circ} \mathrm{C}$ and $1050^{\circ} \mathrm{C}$ for 1 hr, $D_{a b}^{\max } / D_{n}$ is always zero. This suggest that there is no possibility of AGG in the system which is in agreement with experimental observation (see figure 4.10). In the case of the AR state heat treated at $1200^{\circ} \mathrm{C}$ for $1 \mathrm{hr}, D_{a b}^{\max } / D_{n}$ is just below 2 and above $10 \mathrm{sec}$ in the treatment shows a value below 1.5. Although at the initial stage of $1200^{\circ} \mathrm{C}$ heat treatment, AGG might be probable but for a very short period of time ( $<10$ second). Even if AGG occurs at the beginning, the microstructure will soon transforms to NGG with prolonged holding at that temperature (see figure 4.11). The DCM predicts probability of AGG throughout the heat treatment of NT state at $1050^{\circ} \mathrm{C}$. From the experimental observations (shown in figure 4.15) it can be seen that there is pronounced AGG in the microstructure in NT state heat treated at $1050^{\circ} \mathrm{C}$ for 20,40 and $60 \mathrm{~min}$. The model prediction of NGG in NT state heat treated at $900^{\circ} \mathrm{C}$ and $1200^{\circ} \mathrm{C}$ are also in agreement with the experimental observations which indeed indicated NGG (see figure 4.14).

A summary of the all the experimental observations and model predictions on grain growth condition are listed in table 5.2. Soft coupled model (see chapter 4), predicts AGG in NT state heat treated at $900^{\circ} \mathrm{C}$ but microstructural observation shows a NGG microstructure. In the present case of dynamic coupled model, the prediction accuracy has improved, as the model predicts NGG instead of AGG.

\section{Grain growth condition for different heating rates}

The present study is dealing with the prior austenite grain growth condition 
Table 5.2: Steel-A: summary of model predictions and experimental observations of grain growth condition.

\begin{tabular}{|c|c|c|}
\hline State & Model prediction & Experimental observation \\
\hline AR- $900^{\circ} \mathrm{C}(1 \mathrm{hr})$ & NGG & NGG \\
\hline AR- $1050^{\circ} \mathrm{C}(1 \mathrm{hr})$ & NGG & NGG \\
\hline AR- $-200^{\circ} \mathrm{C}(1 \mathrm{hr})$ & NGG & NGG \\
\hline NT- $900^{\circ} \mathrm{C}(1 \mathrm{hr})$ & NGG & NGG \\
\hline NT- $1050^{\circ} \mathrm{C}(1 \mathrm{hr})$ & AGG & AGG \\
\hline NT $-1200^{\circ} \mathrm{C}(1 \mathrm{hr})$ & NGG & NGG \\
\hline
\end{tabular}

in relation with precipitation state. For this reason, precipitation state evolution above the austenite transformation temperature $\left(A C_{1}\right)$ is only considered. It is found by dilatometric analysis that in steel-A, austenite transformation occurs at $800^{\circ} \mathrm{C}$. Considering the transformation temperature, the heating rate effects is simulated between $800^{\circ} \mathrm{C}$ and the target temperature of $1050^{\circ} \mathrm{C}$. This approximation introduces simplicity in calculation but retain the potential to predict the precipitation state evolution in the austenitic phase at different heating rates.

In figure $5.14(\mathrm{a})$, the heat treatment cycles consisting of $0.1,0.5$ and $1.0^{\circ} \mathrm{C} / \mathrm{min}$ heating rates performed on the AR and NT states are shown. Model calculated precipitate volume fractions are shown in figure 5.14 (b) and (c). From figure 5.14 (b), it can be seen that in the AR state, the precipitate volume fraction increases with increasing temperature and then above $950^{\circ} \mathrm{C}$ decreases to reach the equilibrium quantity near $1050^{\circ} \mathrm{C}$. It is noticeable that in $\mathrm{AR}$ state, intermediate increase in precipitate volume fraction is more for slower heating rates in comparison with the faster ones. In all the heating rates, the increase in volume fraction is followed by a decrease when the temperature is above $950^{\circ} \mathrm{C}$. The slower heating rate allowing the precipitation state to get closer to the lower temperature equilibrium which in fact is higher in volume fraction than the final temperature of $1050^{\circ} \mathrm{C}$. Consequently the final precipitate volume fraction for slower heating rate is higher than the faster one.

In the Normalized Tempered (NT) state (see figure 5.14 (c)), there is also an increase in precipitate volume fraction with increasing temperature but only noticeable for the slowest heating rate of $0.1^{\circ} \mathrm{C} / \mathrm{min}$. The effects of heating rate are quite opposite in the NT state in comparison with the AR state. In the NT state, primarily precipitate dissolution is occurred. Here, at the of the temperature ramp, the faster heating rates are showing higher precipitate volume fraction in comparison with the slower one. Again the slower heating rates allowing the precipitation state to get closer to the equilibrium at $1050^{\circ} \mathrm{C}$ thus yielding lower volume fraction. In comparison with the experimental measurement at $1050^{\circ} \mathrm{C}(1 \mathrm{hr}$ of holding), in 

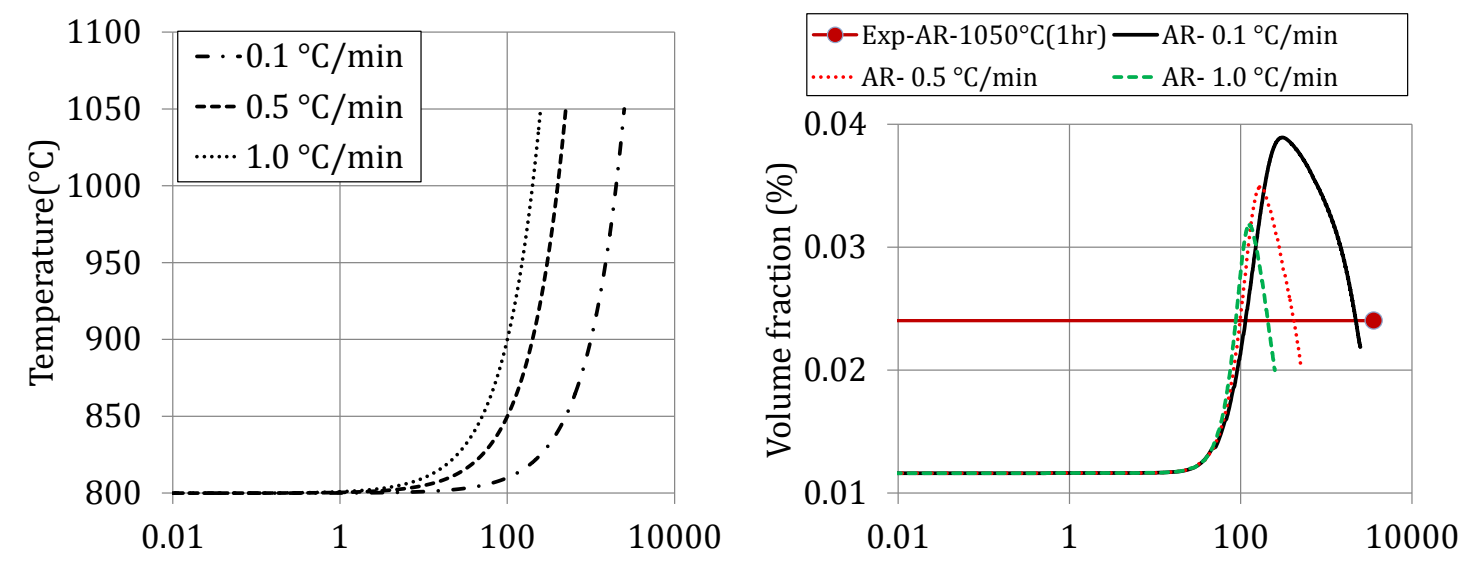

(a) Time(second)

(b) Time(second)

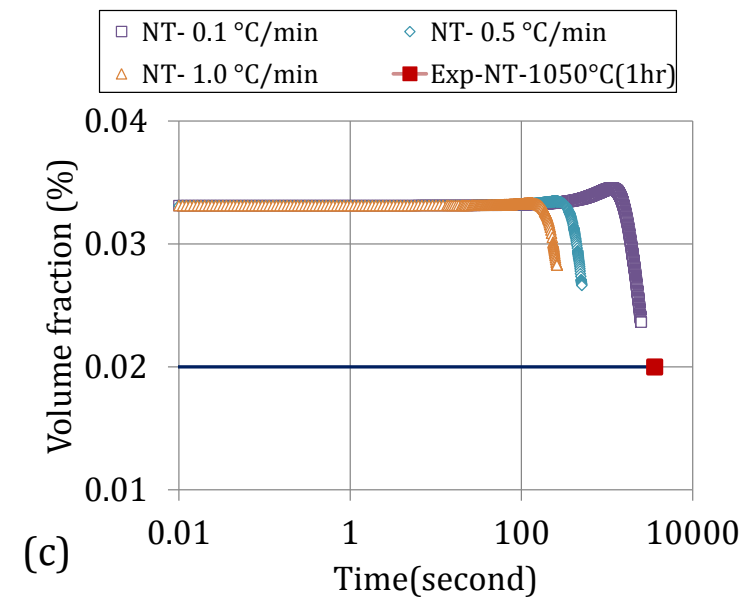

Figure 5.14: (a) $0.1,0.5$ and $1.0^{\circ} \mathrm{C} /$ min heating rates performed on both $\mathrm{AR}$ and NT state, (b) precipitate volume fraction evolution in AR state and (c) precipitate volume fraction evolution in NT state subjected to the heating cycle shown in (a). 


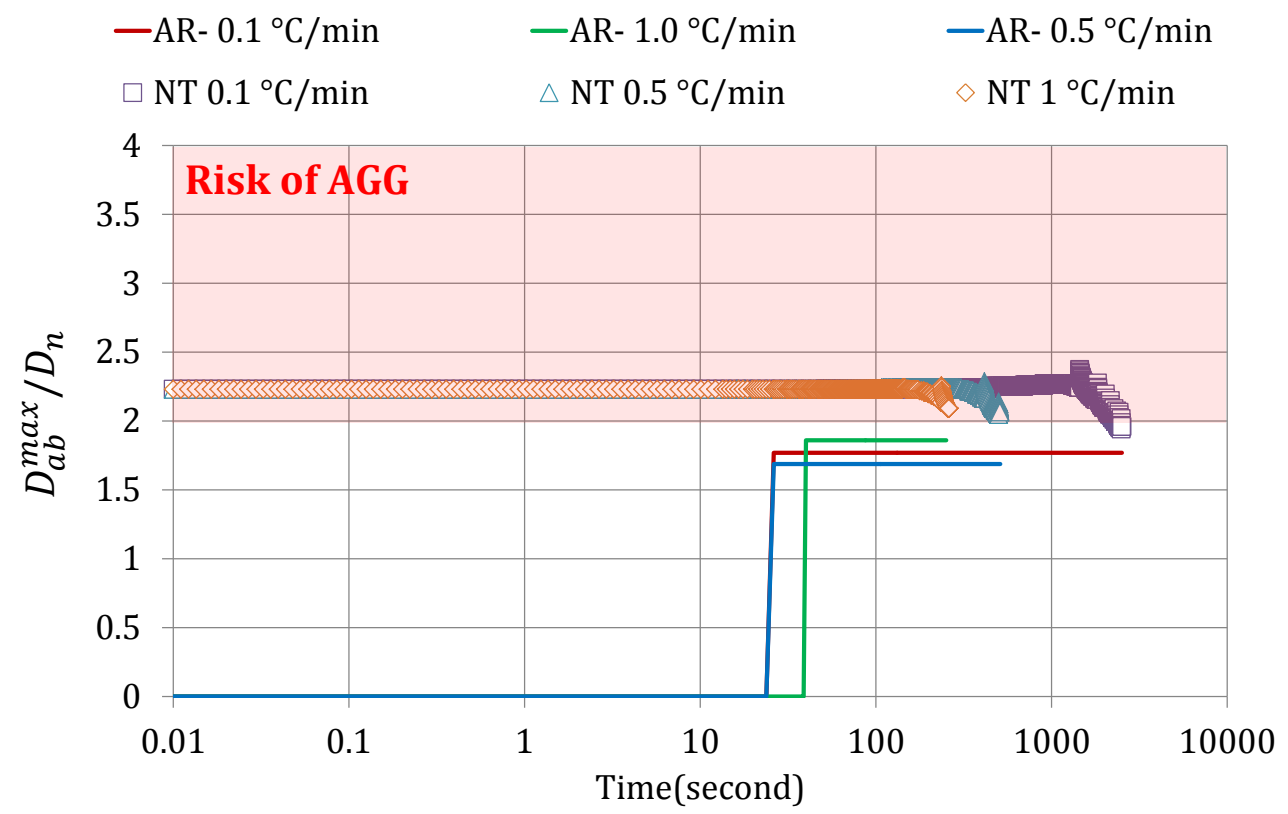

Figure 5.15: Dynamic coupled model prediction of industrial alloy steel-A's AR and NT state subjected to $0.1,0.5$ and $1^{\circ} \mathrm{C} /$ min heating rates. The red shaded zone mark the risk of AGG area for $D_{a b}^{\max } / D_{n}$ value. Here, model parameters $\mu=0.54$ and $\lambda=0.6$.

both the AR and NT states, model prediction for slowest heating rate of $0.1^{\circ} \mathrm{C} / \mathrm{min}$ showing the nearest quantity.

In figure 5.15, the dynamic coupled model predictions of grain growth conditions (AGG or NGG) in the AR and NT states subjected to $0.1,0.5$ and $1^{\circ} \mathrm{C} / \mathrm{min}$ heating rates are presented. From figure 5.15, it can be observed that the AR state subjected to $0.1,0.5$ and $1^{\circ} \mathrm{C} / \mathrm{min}$ heating rates, do not show any probability of AGG. Here, the maximum grain size $\left(D_{n}^{\max }\right)$ with probability to growth relative faster than the mean size grain $\left(D_{n}\right)$ is less than 2 times of $D_{n}$. As the grains with size closer to the mean sized grains grow faster, the microstructure essentially remains quasi-stationary (see figure 5.9). On the other hand, model predictions for the NT state subjected $0.1,0.5$ and $1^{\circ} \mathrm{C} /$ min heating rates show probability of AGG in all the heating rates. In comparison with the experimental observations (see figure 5.10), model prediction of AGG for $0.1,0.5^{\circ} \mathrm{C} / \mathrm{min}$ is in agreement. Although the model is predicting AGG for $1^{\circ} \mathrm{C} / \mathrm{min}$ heating rate, the experimental observation (see figure $5.10\left(C_{1}\right)$ and $\left(C_{2}\right)$ ) is showing normal or no grain growth microstructure. The failure of the model to give a correct prediction in NT state subjected to $1^{\circ} \mathrm{C} / \mathrm{min}$ heating rate, can be due the fact that the model is only capable of giving a qualitative prediction of the grain growth condition. As the kinetics of grain growth condition in terms of size evolution is not calculated by the present approach, the time required for the AGG to propagate is unknown. These 
Table 5.3: Steel-A: summary of model prediction and experimental observation of grain growth condition in samples subjected to different heating rates.

\begin{tabular}{|c|c|c|}
\hline State & Model prediction & Experimental observation \\
\hline AR- $-0.1^{\circ} \mathrm{C} / \min$ & NGG & NGG \\
\hline AR $-0.5^{\circ} \mathrm{C} / \min$ & NGG & NGG \\
\hline AR $-1.0^{\circ} \mathrm{C} / \min$ & NGG & NGG \\
\hline NT- $-0.1^{\circ} \mathrm{C} / \min$ & AGG & AGG \\
\hline NT- $-0.5^{\circ} \mathrm{C} / \min$ & AGG & AGG \\
\hline NT $-1.0^{\circ} \mathrm{C} / \min$ & AGG & NGG \\
\hline
\end{tabular}
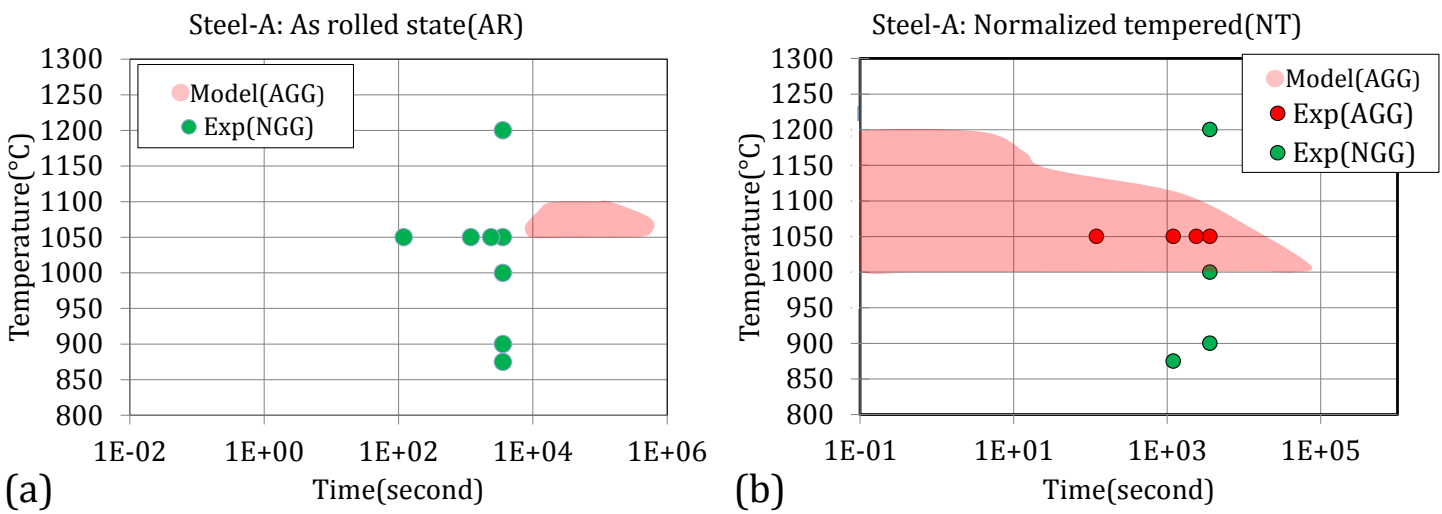

Figure 5.16: Calculated time-temperature mechanism map of grain growth condition in the steel-A: (a) As Rolled (AR) state and (b) Normalized Tempered (NT) state. Abnormal grain growth risk zone is marked with a red shade.

results again highlighted the model's capability and shortcoming to qualitatively predict the grain growth condition (AGG/NGG) in different heating rates.

\subsubsection{Time-Temperature mechanism map of grain growth}

In this section, dynamic coupled model is applied to construct a time-temperature grain growth condition mechanism map. DCM simulation is done starting from $800^{\circ} \mathrm{C}$ till $1300^{\circ} \mathrm{C}$ using a temperature step of $50^{\circ} \mathrm{C}$. The time scales are kept equal for all the simulation. The model predictions are plotted on a time-temperature 2D surface and the AGG risk zone is identified when $D_{a b}^{\max } / D_{n}>2$. In the following, mechanism maps calculated for the AR and NT states are discussed.

In figure 5.16, time-temperature mechanism maps of Abnormal (AGG) and Normal (or No) grain growth (NGG) conditions are presented. In the As Rolled (AR) state (see figure $5.16 \mathrm{a}$ ), model prediction for the heat treatment done at all the temperatures till $10^{4} \mathrm{sec}$ do not show presence of AGG. This is in agreement with the experimental observations, as shown by the superimposed experimental points on the mechanism map. At $1050^{\circ} \mathrm{C}$ and $1100^{\circ} \mathrm{C}$ just above $10^{4}$ second in 
Table 5.4: Composition of industrial alloy steel-B in atomic \%

\begin{tabular}{cccccccc}
\hline $\mathrm{C}$ & $\mathrm{Si}$ & $\mathrm{Mn}$ & $\mathrm{Ni}$ & $\mathrm{Cr}$ & $\mathrm{Al}$ & $\mathrm{Nb}$ & $\mathrm{N}$ \\
0.0963 & 0.352 & 1.22 & 0.133 & 1.23 & 0.0701 & 0.0221 & 0.0929 \\
\hline
\end{tabular}

the heat treatment, the model predicts probability of AGG in the AR state. This indicates at 1050 and $1100^{\circ} \mathrm{C}$, a transition from the NGG to AGG and with longer holding time, again transformation to the NGG microstructure.

A calculated time-temperature mechanism map for the Normalized Tempered (NT) state is shown in figure $5.16 \mathrm{~b}$. In comparison with the AR state, the NT state shows a wide range of temperature and time within which AGG is probable. Model prediction of $\mathrm{AGG}$ at $1050^{\circ} \mathrm{C}$ coincides with the experimental observations for 2, 20, 40 and 60 min of heat treatments. Besides that experimental observation of NGG condition in the heat treated samples of $1200^{\circ} \mathrm{C}, 900^{\circ} \mathrm{C}$ and $875^{\circ} \mathrm{C}$ for $1 \mathrm{hr}$ is also in agreement. Experimental observation in samples heat treated at $1000^{\circ} \mathrm{C}$ for $1 \mathrm{hr}$, shows contradiction with the AGG model prediction. The reason can be related to the small difference between experimentally determined AGG probable temperature $1050^{\circ} \mathrm{C}$ and NGG probable $1000^{\circ} \mathrm{C}$. Although there is a difference in precipitation state at 1000 and $1050^{\circ} \mathrm{C}$, the difference is not large enough to affect the mean field AGG model's outcome. In this particular case, the model is capable of predicting the time-temperature AGG probable zone but incapable of taking into account the AGG and NGG conditions sensitivity with the small change in temperature.

\subsubsection{Industrial alloy: steel-B}

\subsubsection{Composition and heat treatment}

Composition of the industrial alloy steel-B is given in the table 5.4. From the composition it can be seen that there are two major precipitating elements $\mathrm{Nb}$ and Al. Transmission Electron Microscopy (TEM) and also Induction Coupled Plasma (ICP) analysis confirmed the presence of NbC and AlN precipitates in the different heat treated states (for details see appendix A).

Two states of the steel-B are studied: (1) As Rolled (AR) and (2) Isothermal heat treated state (ISO). AR state is obtained by rolling of the as cast section followed by slow air cooling and ISO state is obtained by isothermal heat treatment above $\left(A C_{3}\right)$ and below $\left(A C_{1}\right)$ the austenite transformation temperatures (see figure 5.17). Slow cooling followed by the rolling insured a fully recrystallized microstructure. Isothermal heat treatment of the AR state is done in order to obtain a fully precipitated state. Subsequent heat treatments are performed on the AR 


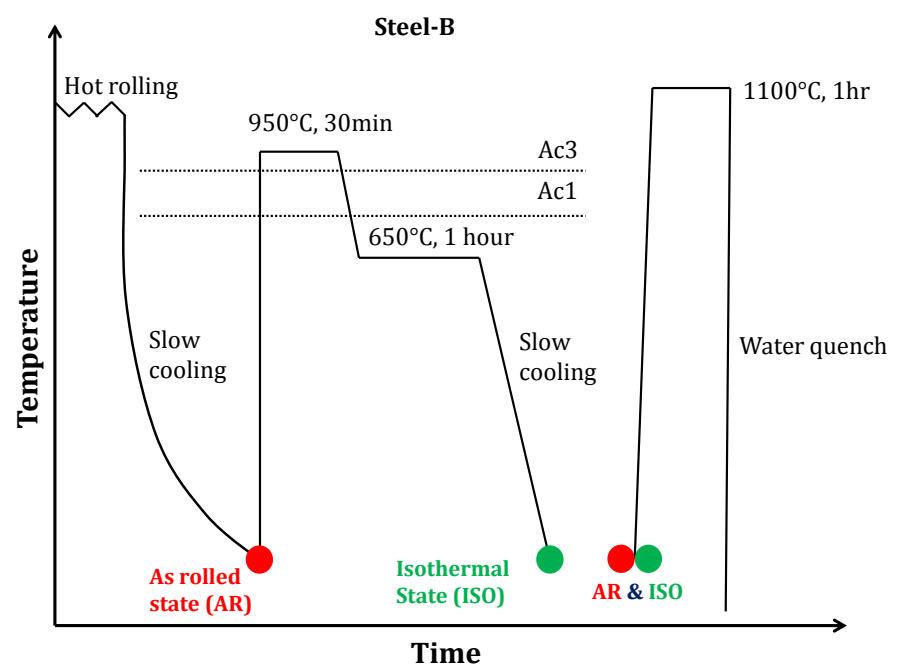

Figure 5.17: Heat treatment cycle of industrial alloy steel-B to obtain As Rolled (AR) and Isothermal heat treated (ISO) state.

and ISO states to realize precipitation state influence on austenite grain growth at different austenitizing temperatures (shown in figure 5.17).

\subsubsection{Precipitation state and grain size evolution}

A detailed discussion on the precipitation states and grain size distributions of the AR and ISO states heat treated at $1100^{\circ} \mathrm{C}$ are presented in chapter 4 section 5.4.2.2.

\subsubsection{Model prediction}

In this section, DCM prediction for the steel-B in isothermally heat treated states are presented. The experimental observation is compared with model prediction and agreement and disagreement between results are discussed.

It is quite essential to predict microstructural properties such as precipitate volume fraction, mean radius and mean grain size to calculate an accurate prediction of the grain growth conditions $(\mathrm{AGG} / \mathrm{NGG})$. In order to validate the coupled modeling, model prediction of precipitate volume fraction, mean radius and mean austenite grain size is presented in figure 5.18. It can be seen from figure 5.18 (a), (b) and (c) that the model predictions of precipitate volume fraction, mean radius and also mean austenite grain size are quite in agreement with the experimental observations. It is already discussed in the earlier section 5.2, that the AGG model prediction is dependent on these physical parameters. So, a reasonable prediction of these physical parameters allow the AGG model to take in to account a close approximation of the microstructural properties such as: precipitation state and 

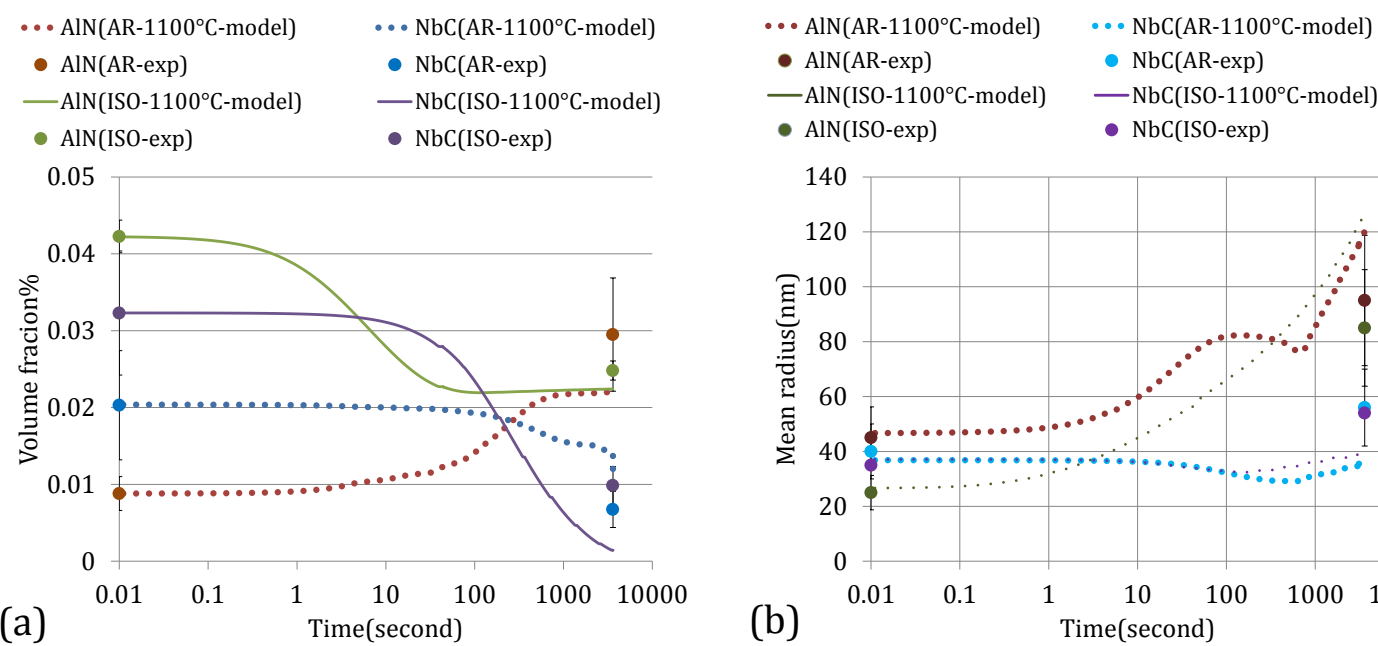

(a)

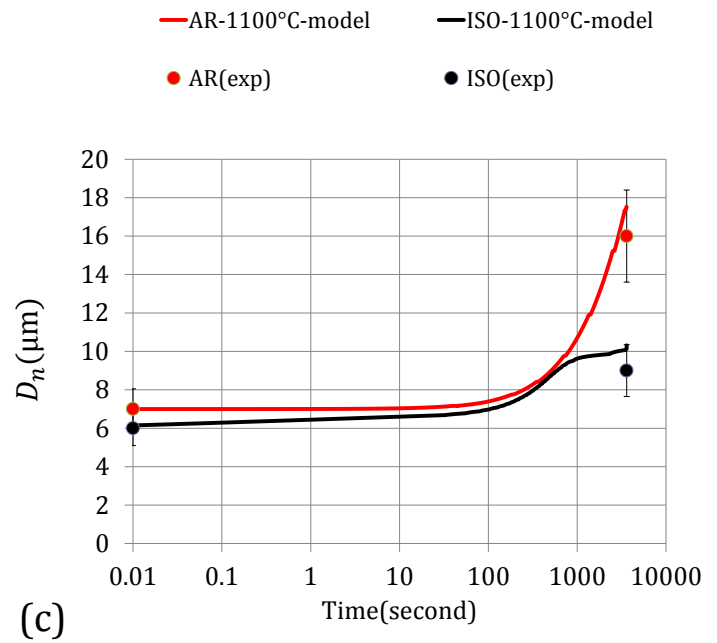

Figure 5.18: Steel-B: AR and ISO state's (a) precipitate volume fraction, (b) precipitate mean radius and (c) mean grain size $\left(D_{n}\right)$ evolution at $1100^{\circ} \mathrm{C}$ are shown. 


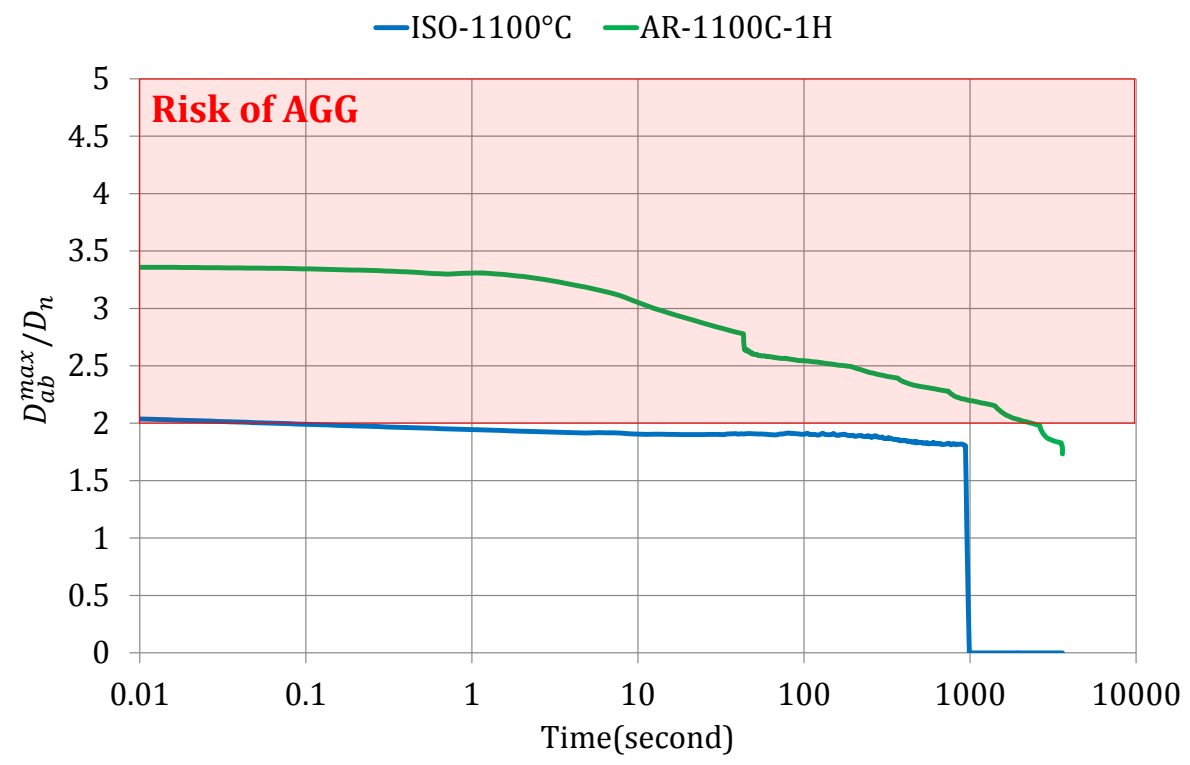

Figure 5.19: Dynamic coupled model prediction of industrial alloy steel-B's AR and ISO state. The red shaded zone mark the risk of AGG area for $D_{a b}^{\max } / D_{n}$ value. Here, model parameters $\mu=0.54$ and $\lambda=0.6$.

Table 5.5: Steel-B: summary of model prediction and experimental observation of grain growth condition.

\begin{tabular}{|c|c|c|}
\hline State & Model prediction & Experimental observation \\
\hline AR- $1100^{\circ} \mathrm{C}(1 \mathrm{hr})$ & AGG & AGG \\
\hline ISO- $1100^{\circ} \mathrm{C}(1 \mathrm{hr})$ & NGG & NGG \\
\hline
\end{tabular}

mean austenite grain size.

Figure 5.19 represents the DCM prediction of grain growth conditions (AGG or NGG) in As Rolled (AR) and Isothermally Treated (ISO) states heat treated at $1100^{\circ} \mathrm{C}-1$ hr. From figure 5.19, we can observed that in the ISO state $D_{a b}^{\max } / D_{n} \approx 2$ which is at the edge of the AGG risk zone. Although the model suggests that there might be a probability of AGG in the system, the experimental results refers Normal Grain Growth (NGG) condition (see figure $4.20\left(A_{2}\right)$ ). In the AR state, the model predicts $D_{a b}^{\max } / D_{n}>3$, which refers to an AGG favorable situation. In the AR state grains larger than 3 times of the mean sized grain have the potential to grow comparatively faster than the others. As faster growth of the larger grains initiate AGG in the microstructure, it is more reasonable to conceive that in the AR state AGG will occur. In fact experimental observation shown in figure $4.20\left(B_{1}\right)$ indeed shows abnormally grown microstructure in the AR state heat treated at $1100^{\circ} \mathrm{C}$ for $1 \mathrm{hr}$.

All the experimental observations and model predictions in the steel-B on grain growth condition are summarized in table 5.5. 

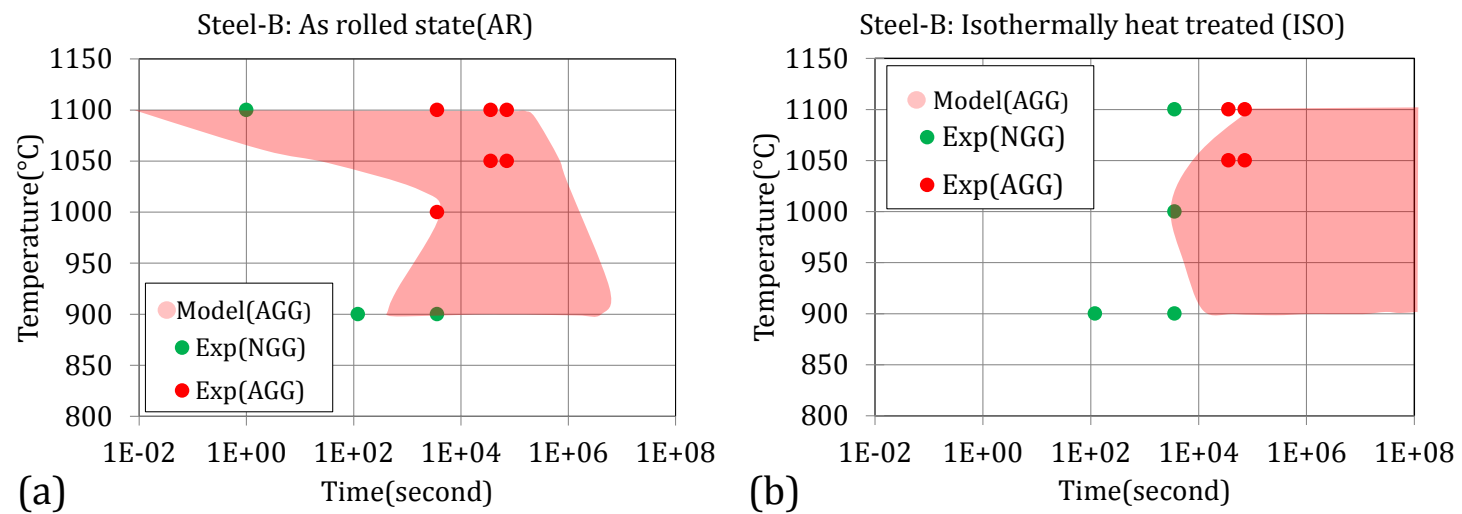

Figure 5.20: Calculated time-temperature mechanism map of grain growth condition in steel-B: (a) As Rolled (AR) state and (b) Isothermally heat treated (ISO) state. Abnormal grain growth probability is marked with a red shaded zone.

\subsubsection{Time-Temperature mechanism map of grain growth}

In this section, dynamic coupled model is applied to construct a time-temperature grain growth condition mechanism map. DCM simulation is done starting from $800^{\circ} \mathrm{C}$ till $1150^{\circ} \mathrm{C}$ using a temperature step of $50^{\circ} \mathrm{C}$. The time scales are kept equal for all the simulation. The model predictions are plotted on a time-temperature $2 \mathrm{D}$ surface and the AGG risk zone is identified when $D_{a b}^{\max } / D_{n}>2$. In the following, mechanism maps calculated for the AR and ISO states discussed.

In figure 5.20, time-temperature mechanism maps of Abnormal (AGG) and Normal (or No) grain growth (NGG) conditions are presented. The model prediction of NGG for AR state heat treated at $900^{\circ} \mathrm{C}$ is in agreement for $120 \mathrm{sec}$ of holding time (see figure $5.20 \mathrm{a}$ ). At higher holding time the model is not in agreement with the experimental observation. This contradiction in results can be correlated to the austenite grain boundary mobility at $900^{\circ} \mathrm{C}$. The pinning condition might facilitate AGG but due to lower mobility of the grain boundary, AGG might not propagate fast enough to create an impact on the microstructure. The AR state at $1100^{\circ} \mathrm{C}$ shows AGG microstructure in heat treatment done for more than $1 \mathrm{hr}$. AGG probability in the AR state disappear after $\sim 10^{4} \mathrm{sec}$ at $1100^{\circ} \mathrm{C}$. So, a transformation from AGG $\rightarrow$ NGG can be observed. The model predicted AGG from the very beginning of the heat treatment, which suggest failure of the current modeling scheme to predict the kinetic nature of the AGG propagation. As stated earlier, the modeling scheme is more oriented towards qualitative prediction of AGG.

The calculated time-temperature mechanism map of grain growth condition of Isothermally heat treated (ISO) state is shown in figure 5.20 (b). It can be observed that at $1050^{\circ} \mathrm{C}$ and $1100^{\circ} \mathrm{C}$ with $10 \mathrm{hr}$ of holding time, the model prediction of AGG is in agreement with the experimental observation. The experimental observations 
Table 5.6: Limiting grain diameter $\left(D_{n}^{l}\right)$.

\begin{tabular}{|c|c|}
\hline Steel-A & $D_{n}^{l}(\mu \mathrm{m})$ \\
\hline As Rolled (AR) & 57 \\
Normalized Tempered state (NT) & 19 \\
\hline \hline Steel-B & $D_{n}^{l}(\mu \mathrm{m})$ \\
\hline As Rolled (AR) & 13 \\
Isothermally heat treated state (ISO) & 10 \\
\hline
\end{tabular}

for $1 \mathrm{hr}$ holding at $900^{\circ} \mathrm{C}$ and $1000^{\circ} \mathrm{C}$ temperature show NGG microstructure, but the model is predicting AGG. As stated earlier, the model is not capable of predicting kinetics of AGG propagation but only the probability of a certain pinning condition impact on grain growth. Although the model prediction give an insight in the material grain structure's probable evolution, the time required for evolution is can not be predicted. This is the reason, why the model predictions at shorter holding time show disagreement with the experimental observation.

\subsubsection{Comparison between industrial alloys mechanism maps}

A comparison between the mechanism maps of steel-A and steel-B shows quite different grain growth conditions in the as rolled state. The reason can be correlated to the precipitate volume fraction in the alloys. Steel-B ( $\mathrm{Al}$ and $\mathrm{Nb}$ ) contains approximately two times of precipitating elements than the steel- $\mathrm{A}(\mathrm{Al})$. In fact, the steel-A's as rolled state contains $0.012 \%$ whereas the steel-B's contains $0.03 \%$ ( $\sim 3$ times of steel-A) total precipitate volume fraction. The effects of higher precipitate volume fraction in steel-A and steel-B are apparent from the limiting grain diameters (see table 5.6). The AR state in steel-A shows $57 \mu \mathrm{m}$ and in steel-B it is $13 \mu \mathrm{m}$, which is 4.4 times smaller than steel-A. Smaller limiting grain size in steel-B's AR state suggests a much higher pinning pressure in comparison with steel-A. So, in the steel-B the AGG risk region is approached from a moderate pinning condition and in the steel-A it is approached from a low pinning condition. This is the reason why in the steel-A's AR state shows mostly NGG but in the steel-B's AR state AGG is quite prominent.

The fully precipitate states: (I) NT in steel-A and II ISO in steel-B, also showed quite different grain growth conditions in relation with temperature and time. The NT state (steel-A) shows probability of AGG between 1000 and $1200^{\circ} \mathrm{C}$ at the beginning of heat treatment. On the other hand, ISO state (steel-B) shows AGG probability at higher holding time $>10^{3} \mathrm{sec}$. Steel-A's NT state contains $0.033 \%$ whereas the steel-B's contains $0.074 \%$ ( 3 times of steel-A) total precipitate volume fraction. The NT state in steel-A shows $19 \mu \mathrm{m}$ and the ISO state in 
steel-B it is $10 \mu \mathrm{m}$ mean austenite grain diameter, which is $\sim 2$ times smaller than steel-A. Smaller limiting grain size in steel-B's NT state suggests a much higher pinning pressure in comparison with steel-A. Moderate pinning condition in the NT state allows the pre-existing larger grains in the microstructure to grow comparatively faster and initiate AGG. Fully precipitated ISO state has very high pinning condition which prevents AGG at the beginning of heat treatments. Higher holding time allows the precipitation state to coarsen and allows AGG onset. These differences in grain growth condition in two alloys rather confirms the AGG mechanism map presented in the chapter 2. As shown in the mechanism map AGG can be approached either from very highly pinned situation or at moderate pinning conditions. A low pinning condition $\left(D_{n} / D_{n}^{l}<0.5\right.$ ratio between mean grain size and limiting grain size) which allows the grains to grow and can effectively prevent AGG. From the perspective of steel-B's ISO state, a very highly pinned condition $D_{n} / D_{n}^{l}>0.9$ can also prevent AGG by creating a immobile grain structure.

\subsection{Conclusions from this chapter}

In this chapter, a dynamic coupled modeling approach of precipitation and abnormal grain growth model is presented. The model prediction sensitivity to different precipitation state evolution in low alloy steel is also studied. The model is tested for two industrial alloys steel-A and steel-B and comparison with experimental observations are explained. The main conclusions that can be obtained from this chapter are listed below:

- Dynamic coupled model follow the unidirectional data flow method between precipitation and abnormal grain growth model. Here in the realm of homogeneous precipitate nucleation and growth, calculated precipitation state from precipitation model is feed into the AGG model. The AGG model calculates the precipitate induced pinning using the precipitate size distribution and finally give prediction on grain growth condition (AGG or NGG) and also mean grain size evolution.

- In the present study, heterogeneous precipitate growth is not taken into account due to the mean grain size being large enough to have negligible effects caused by heterogeneous precipitate growth on the overall precipitation kinetics. Dynamic coupled modeling approach have the provision to take into account the homogeneous and heterogenous precipitate growth kinetics and the evolving mean grain size. It should be mentioned here that heterogeneous precipitate growth with evolving mean grain size is rather a complex system where number of precipitate reside in the grain boundary always changes.

- A theoretical study using four precipitation state evolution: (1) AGG in pres- 
ence of a stable precipitate, (2) AGG in presence of precipitate coarsening, (3) AGG in presence of precipitate nucleation and (4) AGG in presence of precipitate dissolution gave us the following understandings:

1. In a constant precipitate volume fraction, number density and mean radius, an increase in mean grain size increases AGG probability.

2. In a precipitate coarsening state, decrease in precipitate number density (constant volume fraction), decreases the AGG probability.

3. A certain quantity of precipitate volume fraction and number density is required for AGG to occur.

4. The AGG probability disappear with decreasing precipitate volume fraction and number density (dissolution).

- The model is verified using experimental observation from two industrial alloys: (1) Steel-A and (2) Steel-B. It is observed that the model prediction for isothermal heat treatment is in agreement where the holding time at a particular temperature is quite high $(\geq 1 \mathrm{hr})$. In the case of shorter holding time $(<1 \mathrm{hr})$, in some heat treated states the predictions are not in agreement with the experimental observations.

- The model prediction for different heating rates also showed quite good agreement with the experimental observation. This emphasize the model capability in predicting grain growth condition not only in isothermal condition but also in non-isothermal conditions.

- The results presented in this chapter show that the dynamic coupled modeling approach is capable of producing comprehensive time-temperature relationship with the grain growth conditions. It is proven to be a convenient tool to identify and avoid abnormal grain growth in alloys without spending resources on expensive and time consuming experiments. 
Cette thèse est accessible à l'adresse : http://theses.insa-lyon.fr/publication/2013ISAL0094/these.pdf () [M.A. Razzak], [2013], INSA de Lyon, tous droits réservés 


\section{Chapter 6}

\section{Conclusions and Perspectives}

\section{Summary and conclusions}

Abnormal Grain Growth (AGG) has been investigated for decades, and yet the phenomenon itself is not fully understood. There are still arguments about the root cause of AGG in low alloy steels. For this reason, further experimental and also numerical modeling work is required to understand and draw relationship of AGG occurrence with the thermo-mechanical treatment. In order to extend the present knowledge, the main objective of this thesis is formulated to understand the Prior Austenite Grain (PAG) growth behavior in low alloy steel. Normal and Abnormal Grain growth (AGG) initiation and propagation of PAG's, correlation with precipitation state is investigated. The investigation is extended from experimental work to numerical modeling. The main results from the thesis are summarized in the next paragraph.

An abnormal grain growth model using simplified assumption is presented in the chapter 2. Abnormal grain growth pressure is calculated using simple geometrical consideration, where a comparatively large grain is surrounded by much smaller ones. Significant assumptions include: The grain growth is driven by decrease in interfacial energy, evenly distributed precipitates tend to retard the grain boundary movement thus pinning the boundary, pinning occurs both by the precipitates lying on the grain boundary and also located in the corner or quadruple points. A comparative study of precipitate size distribution's impact on Zener and corner point pinning is presented. The study refers to the fact that whole precipitate distribution consideration instead of mean radius, shows an overall increase in calculated Zener and corner pinning. It is shown that AGG is possible when the larger grains in the microstructure can grow comparatively faster than the smaller or mean sized ones. This analogy lead to the condition of AGG initiation and propagation in the microstructure $\left(d D_{a b} / d t>0\right.$ and $\left.d / d t\left(D_{a b} / D_{n}\right)>0\right)$. Finally, the model is tested using experimental results from a model alloy (FeVNbCN) and 
it has been proven that the model is capable of predicting AGG and Normal Grain Growth (NGG) presence in the microstructure with constant model parameters.

In chapter 3, a precipitation model based on Classical Nucleation and Growth Theories (CNGTs) is presented. The model is effective in predicting precipitation state evolution with time and temperature, provided with accurate thermodynamic description of the precipitating phase. The required parameters for modeling are either available in the literature or experimentally measurable. The experimentally measurable model parameters include: (i) precipitate volume fraction by ICP analysis, (ii) precipitate chemistry by EDS and (iii) size distribution by High Angle Annular Dark Field (HAADF) mode in Transmission Electron Microscope (TEM). Precipitation state evolution is investigated considering both homogeneous precipitate growth in the matrix and heterogeneous growth of precipitate lying in the grain boundary or quadruple point. It has been found, that only small fraction $(<0.5 \%)$ of the total precipitate number can lie in the grain boundaries, which in fact cause a negligible effect on the overall precipitation kinetics. The precipitation model is applied to two industrial alloys: (1) steel-A and (2) steel-B. In comparison with the experimental results, the model predictions showed reasonable agreement in terms of precipitate volume fraction, mean radius and size distribution.

In chapter 4, a coupled modeling is presented where the Abnormal Grain Growth (AGG) and precipitation models are coupled softly. Here, the term soft coupling emphasizes the fact that precipitation and AGG models are executed on separate time scale. First, precipitation model is executed in order to calculate the precipitate size distribution evolution at each time step $\left(N_{i} R_{i}(t)\right)$. Then the calculated precipitate size distribution is fed in the AGG model to calculate the AGG probability. One of the significant characteristics of soft coupled approach is that, the mean grain size is kept constant. This helped us investigate only the effects of precipitation state evolution on grain growth condition. From this modeling approach, the following significant relationships between precipitation state and AGG have been found: A decrease in precipitate number density (constant volume fraction), decreases the AGG probability, (B) a certain volume fraction and number density of precipitate is required to induce AGG in the microstructure and (C) AGG probability disappear with decreasing precipitate volume fraction and number density (dissolution). The soft coupled model is applied to two industrial alloys: (1) steel-A and (2) steel-B. Model predictions are compared with the experimental observations. In comparison with the experimental observations, the model is predictions of AGG and Normal or No Grain Growth (NGG) are quite in agreement. Disagreement between experimental observation and model prediction is found where the mean austenite grain size evolution is considerable in a heat treatment. 
In the chapter 5, a coupled modeling is presented where the Abnormal Grain Growth (AGG) and precipitation models are coupled dynamically. Here, the term dynamic coupling emphasizes the fact that the precipitation and abnormal grain growth models are executed on the same time scale. In this modeling approach, calculated precipitate size distributions are fed in the AGG model to predict AGG probability in the same time step. Moreover, in the dynamic coupled modeling, mean grain size $\left(D_{n}\right)$ evolution with time is also calculated which is quite significant in the present mean field approach. This allows the model to take in to account change in the number fraction of precipitates lying at the grain boundaries and quadruple points. This can in fact change the number of precipitate experiencing heterogeneous growth or dissolution. It should be mentioned here that in the present study, heterogeneous precipitate growth or dissolution is not considered. Because of the mean grain size in the studied low alloy steels being large enough $(>5 \mu \mathrm{m})$, not to have significant contribution from heterogenous growth in overall precipitation kinetics. Dynamic coupled modeling approach found to be suitable for constructing a comprehensive time-temperature grain growth condition mechanism maps. Application to two industrial alloys: (1) steel-A and (2) steel-B, showed agreement between observed grain growth conditions (AGG/NGG) and model predictions at different isothermal and non-isothermal heat treatments. Thus the dynamic coupled model is capable of predicting grain growth conditions at different austenitizing temperature and time, with experimental parameters such as: initial precipitate size distribution and mean grain size. This enables us to design heat treatment cycles which can effectively prevent AGG in the microstructure.

\section{Future work}

In the present work, efforts are given to achieve a more detailed understanding of relationship between Abnormal Grain Growth (AGG) and precipitation state in low alloy steels. The direction of the work is towards qualitative prediction of AGG in relation with precipitation state than quantitative prediction of the microstructure. For this reason, microstructural properties such as disorientation dependent austenite grain boundary energy, change in grain boundary mobility due to solute drag and effects of texture are not taken in to consideration. Although the present coupled modeling approach can give reasonable predictions of AGG, the kinetics of grain size distribution evolution can not be predicted. So, in future, the present coupled modeling can evolve by incorporating the grain size distribution evolution kinetics. The following studies can be considered to extend the present understanding and build up a comprehensive frame work of AGG modeling.

- The austenite grain boundary energy in relation with the misorientation 
between grains can be measured, by the experimental technique like grain boundary grove analysis using Atomic Force Microscope (AFM) [62] . Besides that, molecular dynamics study on FCC iron's bi-crystal can also give useful approximation in misorientation dependent grain boundary energy and mobility $[49,23]$. These methods can be used to find out anisotropic grain boundary energy and mobility in FCC iron and utilized in phase filed or Monte-Carlo type study to find out its effects on the AGG.

- It is reported by S.G. Kim [37] and other authors that the solute drag can change the grain-growth mode in presence of texture, anisotropy of the grain boundary mobility, pinning by the second phase particles and also grain size advantage. Temperature dependency of the solute drag effects can be incorporated in the present scheme, to study the comparative dominating mechanism in AGG initiation and propagation kinetics.

In the present modeling approach, the initial austenite $(\gamma)$ grain size is measured experimentally and then taken as input to model the effects of heat treatment on grain growth condition. This method is proven to be effective in predicting AGG in isothermal and non-isothermal heat treatment provided with the experimentally measured initial mean $\gamma$ grain size. In a situation where the material is heated to a desired austenitizing temperature using different heating rates, mean $\gamma$ grain size will change drastically due to $\alpha$ or $\alpha^{\prime}$ to $\gamma$ transformation kinetics. In order to model the effects of different heating rates on the AGG, it is necessary to measure the initial (just after the $\alpha$ or $\alpha^{\prime}$ to $\gamma$ transformation is complete) mean $\gamma$ grain size for each studied heating rate. The need for large amount of experimental measurements for different heating rates, can be overcome by introducing $\alpha$ or $\alpha^{\prime}$ to $\gamma$ phase transformation model. A phase transformation model based on the classical nucleation and growth theories can be chosen in this regard [13]. Besides the CNGTs based model, phases field model [6] can also be utilized to predict the austenite grain nucleation and growth kinetics during transformation. Introduction of initial austenite grain size prediction will lead the modeling approach to a frame work of materials microstructure prediction and consequently, will enable us to design custom materials microstructure with desired properties. 
Cette thèse est accessible à l'adresse : http://theses.insa-lyon.fr/publication/2013ISAL0094/these.pdf () [M.A. Razzak], [2013], INSA de Lyon, tous droits réservés 


\section{Appendix A}

\section{Experimental techniques}

\section{Precipitate volume fraction measurement by ICP:}

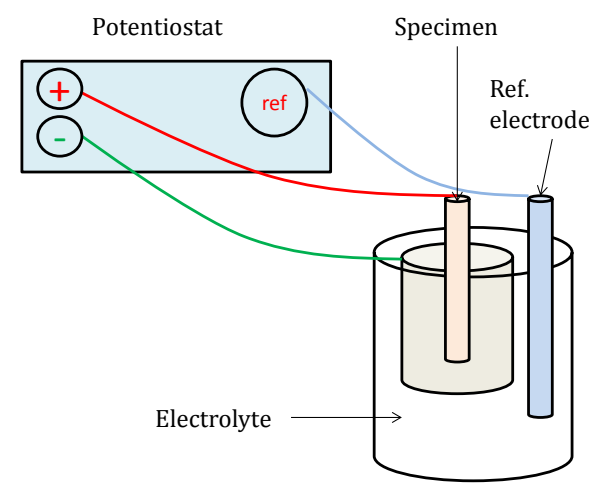

Figure A.1: A schematic representation of electrolytic dissolution of specimen.

Precipitate volume fraction is measured using Induction Coupled Plasma (ICP) measurement technique. The sample's matrix is first dissolved in a solution of salicylic acid, lithium chloride and methanol (as shown in the figure A.1). As the precipitates do not dissolved in the solution, filtration separates the precipitates from the solution. Separated precipitates are then dissolved in a high acidic solution consisting of $\mathrm{HNO}_{3}+\mathrm{HCL}+\mathrm{HF}+\mathrm{H}_{2} \mathrm{SO}_{4}$. Precipitates dissolved in the solution are then analyzed in the ICP and the precipitates volume fraction is measured in terms of the substituting solute elements (i.e. Al, Nb, V, Cr, etc) in Part Per Million (PPM). The substitutional solute element's mass fraction is converted to the constitutive precipitate's volume fraction using the expression A.1.

$$
f_{\nu}^{P}(T, t)=f_{m}^{A}(T, t) \frac{\rho_{M}}{\rho_{A_{x} B_{y}}}\left(\frac{x}{y} \frac{M_{B}}{M_{A}}+1\right)
$$


Here, $f_{m}^{A}(T, t)$ is the measured mass fraction of the substitutional element $A$ of a precipitate $A_{x} B_{y}, \rho_{M}$ is the molecular density of the matrix, $\rho_{A_{x} B_{y}}$ is the molecular density of the precipitate, $M_{A}$ is the molar mass of element $A, M_{B}$ is the molar mass of element $B$ and $f_{\nu}^{P}(T, t)$ is the precipitate volume fraction.

\section{Precipitate size measurement:}

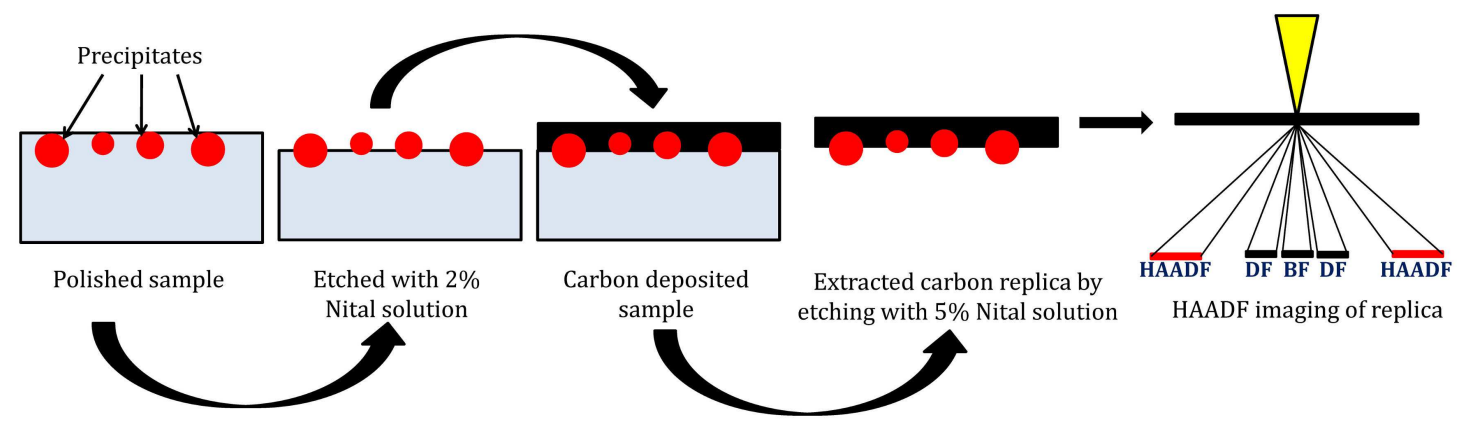

Figure A.2: A schematic representation of carbon extraction replica preparation.

Precipitate size distribution and chemistry in a particular heat treated state are determined using Transmission Electron Microscope (TEM) on the carbon extraction replica. A schematic representation of the step by step process starting from sample preparation to replica analysis is presented in the figure A.2. The process can be summarized as follows:

1. First the samples are polished till $1 \mu \mathrm{m}$ diamond polishing medium to obtain a mirror like surface.

2. Polished sample is then etched with $2 \%$ nital solution $(2 \%$ by volume Nitric acid in Ethanol) to expose the precipitate-matrix interface.

3. Etched sample is then coated with $\sim 20 \mathrm{~nm}$ of carbon by sputtering technique using SCD500 BALTEC sputtering machine.

4. Carbon coating on the sample is cut into approximately $3 \times 3 \mathrm{~mm}^{2}$ squares, which is small enough to fit on the copper grid having a 200 mesh size.

5. The sample is then placed in a $5 \%$ nital solution ( $5 \%$ by volume Nitric acid in ethanol) bath until the sliced carbon film delaminated from the sample's surface. Delaminated film slices are then passed through three baths of methanol-ethanol-methanol to remove traces of acid and finally the washed slices are placed on copper grids. 
6. The prepared carbon extraction replicas are then examined in a JEOL2010 TEM with a Field Emission Gun (FEG), using the High Angle Annular Dark Field (HAADF) mode. An acceleration voltage of $200 \mathrm{kV}$ was used.

7. Images taken in HAADF mode are analyzed by image analysis software ImageJ to measure the equivalent sphere's radius.

\section{Grain size measurement:}

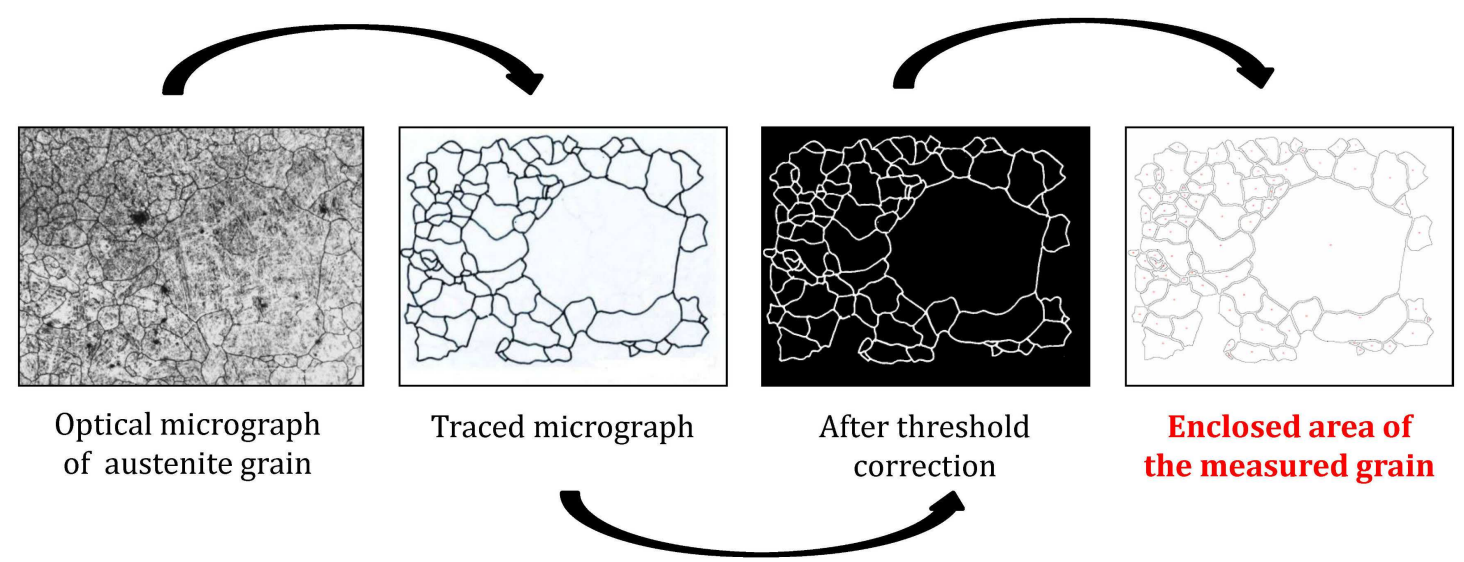

Figure A.3: A schematic representation of prior austenite grain size measurement.

Hot picric acid solution $\left(\sim 80^{\circ} \mathrm{C}\right)$ modified with surface reactant is used to reveal the prior austenite grain boundary in this study $[72,12,14]$. A schematic representation of all the steps to measure the grain size are given in the figure A.3. The grain size measurement technique can be summarized as follows:

1. Samples heat treated at different austenitic temperatures are quenched in order to retain impurity segregation at the prior austenite grain boundaries. The samples are then polished from rough silica paper till $1 \mu \mathrm{m}$ diamond polishing medium to obtain a mirror like surface.

2. The polished samples are placed in a solution of hot picric acid modified with surface reactant in order to reveal the prior austenite grain boundaries.

3. After the etching, optical micrography is performed on the etched surface to acquire images containing austenite grains. In most cases, picric acid etching does not produce a clean microstructure with noticeable difference in contrast between matrix and austenite grain boundaries. For this reason, hand tracing is required to clearly delineate the grain boundaries.

4. Hand traced image is then scanned and treated with image processing software like 'ImageJ' to measure the grain size in the sample. Large quantity 
of measurements are required to build a representative picture of the grain sizes in the sample. 
Cette thèse est accessible à l'adresse : http://theses.insa-lyon.fr/publication/2013ISAL0094/these.pdf () [M.A. Razzak], [2013], INSA de Lyon, tous droits réservés 


\section{Appendix B}

\section{A Monte-Carlo study of the}

\section{compact efficiency factor $\mu$}

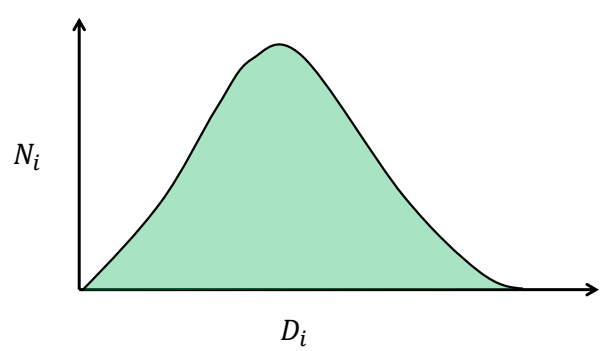

Sample grains from a normal grain size distribution in increasing diameter

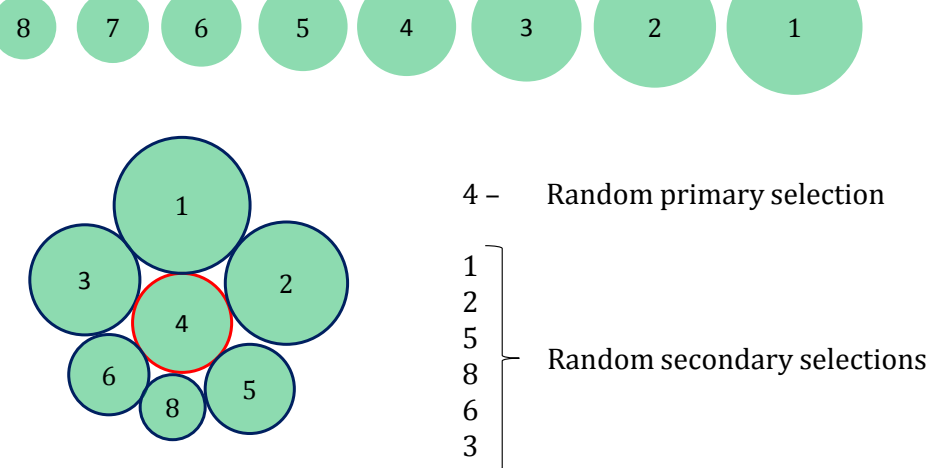

Figure B.1: A schematic representation of the Monte-Carlo study of the compact efficiency factor $\mu$.

The compact efficiency factor $(\mu)$ refers to the compactness of a grain size distribution or how well grains are distributed in close proximity, in a given system. In the present study, the compact efficiency factor determines the total number of grains surrounding a relatively larger grain and as a consequence the driving force 
for growth. So, it is quite important to calculate the envelop of $\mu$ values which represents the real system. A Monte-Carlo (MC) type study is found to be appropriate in this regard, as the random selection of grain size arrangement has high probability of representing different possible configurations in the microstructure. Large number of MC steps and an average over all the MC steps should give us an realistic approximation of the compact efficiency in the system. A schematic of the MC type study is given in the figure B.1. The calculation procedure can be described as follows:

1. First, a primary grain is selected. As shown in figure B.1, here the primary randomly selected grain is 4 with a radius of $r_{4}$. The total surface area of the primary grain assuming it is a sphere can be expressed as $S_{P}=4 \pi\left(r_{4}\right)^{2}$.

2. In the second step, secondary grains which will arranged around the primary grain are selected randomly. After every single selection, uncovered surface area $\left(S_{U S}\right)$ of the primary grain is checked by subtracting the secondary grain's projected area $\left(S_{S_{i}}=\pi\left(r_{S_{i}}\right)^{2}\right), S_{U S}=S_{P}-\sum S_{S_{i}}$. The random selection of secondary grains are continued till $S_{U S}>0$, this mean if any subsequent randomly selected secondary grain has larger projected surface area then what is available, the selected grain is rejected and the second step is terminated.

3. In the chapter 2 , it is shown that compact efficiency factor $\mu=\rho / 4 \lambda_{P}$ where $\lambda_{P}=1 / 4$ for spherical grain. So, $\mu=\rho$, a ratio between the uncovered surface area of the primary grain $\left(S_{U S}\right)$ and total surface area of $S_{P}$ should provide us with approximate compact efficiency of that particular arrangement.

4. Finally step 1 to 3 is repeated for sufficient times until the standard deviation in calculated $\mu$ is $<0.0001$.

In the figure B.2, average value of $\mu$ evolution with increasing MC step is shown for three different run. It can be observed that at the beginning of the MC run there is a large scatter in data. Even with large initial scatter in date range, with increasing random grain configuration study, the $\mu$ converge to $0.56 \pm 0.02$. It should be mentioned here that the same method is applied other states of the steel-A and similar values are observed. 


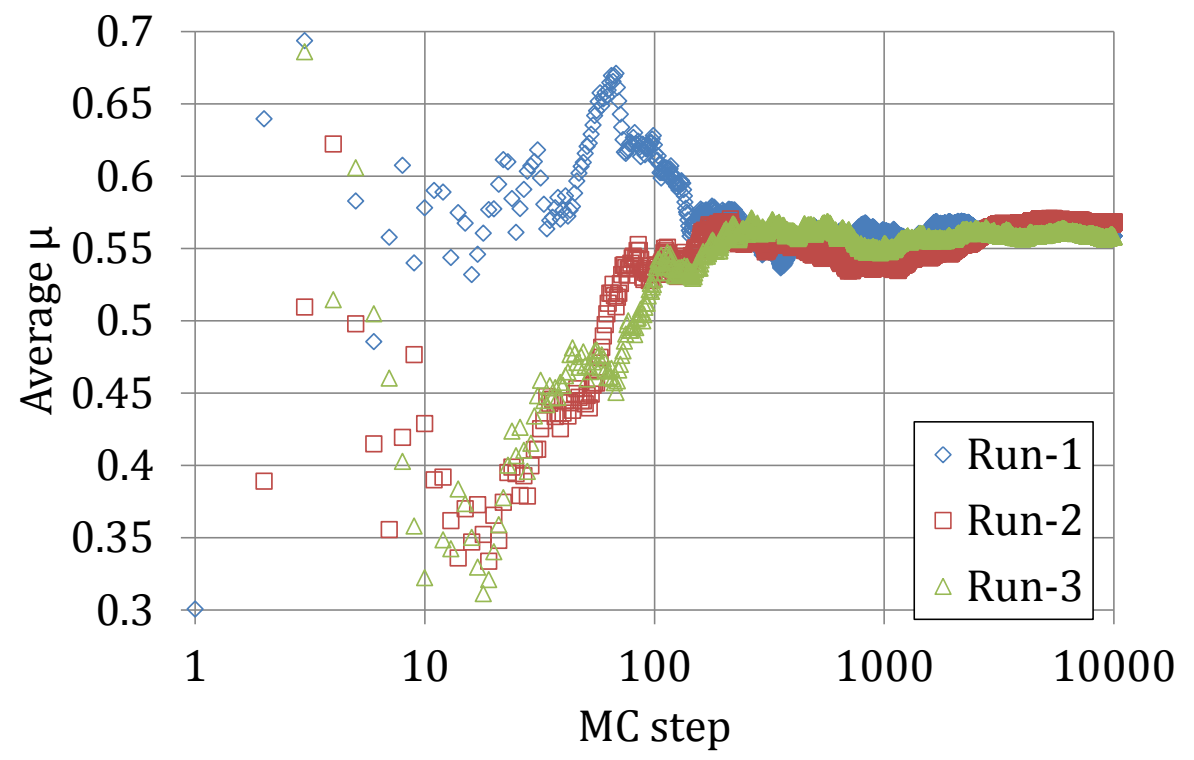

Figure B.2: Average value of compact efficiency factor $(\mu)$ evolution with MonteCarlo step in steel-A's Normalized Tempered (NT) state. 
Cette thèse est accessible à l'adresse : http://theses.insa-lyon.fr/publication/2013ISAL0094/these.pdf () [M.A. Razzak], [2013], INSA de Lyon, tous droits réservés 


\section{Bibliography}

[1] G. Abbruzzese and K. Lücke. A theory of texture controlled grain growth-I. derivation and general discussion of the model. Acta Metallurgica, 34(5):905914, 1986. 71, 101

[2] M. Abramowitz and I.A. Stegun. Handbook of Mathematical Functions. Dover,New York, 1965. 43

[3] D. Acevedo. Evolution de l'état de précipitation au cours de l'austénitisation d'aciers microalliés au vanadium et au niobium. PhD thesis, INSA Lyon, 2007. 2

[4] KA Alogab, DK Matlock, JG Speer, and HJ Kleebe. The Effects of Heating Rate on Austenite Grain Growth in a Ti-modified SAE 8620 Steel with Controlled Niobium Additions. ISIJ International, 47(7):1034-1041, 2007. 117

[5] I. Andersen, O Grong, and N. Ryum. Analytical modeling of grain growth in metals and alloys in the presence of growing and dissolving precipitates-II. Acta Metallurgica et Materialia, 43:2689-2700, 1995. 6, 7, 12, 70, 71, 100, 101

[6] Hamid Azizi-Alizamini. Austenite formation and grain refinement in C-Mn steels. PhD thesis, The University of British Coumbia, 2010. 137

[7] K. Balasubramanian and J.S. Kirkaldy. Experimental investigation of the thermodynamics of Fe-Nb-N austenite and nonstoichiometric niobium nitride (1373-1673 k). Canadian Metallurgical Quarterly, 28:301-315, 1989. 57

[8] R. Becker and W. Döring. Kinetische behandlung der keimbildung in übersättigten dämpfen. Annalen der Physik, 416:719-752, 1935. 37

[9] Y. Bréchet and M. Militzer. A note on grain size dependent pinning. Scripta Materialia, 52:1299-1303, 2005. 6, 7, 13, 14, 26

[10] Leon M. Cheng, E. Bruce Hawbolt, and T. Ray Meadowcroft. Modeling of dissolution, growth and coarsening of aluminium nitride in low-carbon steels. Metallurgical and Materials Transactions A, 21A:1907-2000, 1999. 41, 42

[11] L.M. Cheng, E.B. Hawbolt, and T.R. Meadowcroft. Canadian Metallurgical Quaterly, 39(1):73, 2000. 57 
[12] K.S. Cho, H.S. Sima, J.H. Kima, J.H. Choia, K.B. Leea, H.R. Yangb, and H. Kwona. A novel etchant for revealing the prior austenite grain boundaries and matrix information in high alloy steels. Materials Characterization, 59:786-793, 2008. 140

[13] J. W. Christian. The Theory of Transformation in Metals and alloys. Pergamon Oxford, 1981. 137

[14] C. García de Andrés, M.J. Bartolomé, C. Capdevila, and V. López D. San Martín, F.G. Caballero. Metallographic techniques for the determination of the austenite grain size in medium-carbon microalloyed steels. $M a$ terials Characterization, 46:389-398, 2001. 140

[15] Ö. N. Dogan, G. Michael, and H. Kwon. Metallurgical Transactions A, 23A:2121, 1992. 57

[16] L.A. Erasmus. Japan Iron and Steel Institute, 202:32, 1964. 57

[17] J. C. Fisher. Calculation of diffusion penetration curves for surface and grain boundary diffusion. Journal of Applied Physics, 22:74 - 77, 1951. 40, 46

[18] J. Fridberg, L-E. Törndahl, and M. Hillert. Jernkont. Ann., 153:263-276, 1969. 60,61

[19] J.W. Gibbs. Collected Works, chapter On the equilibrium of heterogeneous substances (1876). Green and Co., 1928. 38

[20] T. Gladman. On the theory of the effect of precipitate particles on grain growth in metals. Proceedings of the Royal Society of London. Series A, Mathematical and Physical Sciences, pages 298-309, 1966. 7, 11

[21] T. Gladman. The physical metallurgy of microalloyed steels. London: The Instute of Materials, 2002. 6, 7, 10, 61

[22] T. Gladman and FB Pickering. Grain-coarsening of austenite. Iron Steel Institute Japan, 205, 1967. 57

[23] G. Gottsteina, D.A. Molodova, L.S. Shvindlermana, D.J. Srolovitzc, and M. Winninga. Grain boundary migration: misorientation dependence. Solid State and Materials Science, 5:9-14, 2001. 137

[24] M. Hillert. On the theory of normal and abnormal grain growth. Acta Metallurgica, 13:227-238, March 1965. 7, 11

[25] Mats Hillert and Stefan Jonsson. An assessment of the Al-Fe-N system. Metallurgical Transactions A, 23A:3141-3149, 1992. 57

[26] K.E. Höner and S. Baliktay. Gießerei-Forschung, 30(2):53, 1978. 57

[27] T.H. Hoogendorn and M.J. Spanraft. In Microalloying 75, Washington, DC, 1975, Union Carbide Corporation, New York, NY, 1977. 57 
[28] J. J. Hoyt. On the coarsening of precipitates located on grain boundaries and dislocations. Acta Metallurgica et Materialia, 39 (9):2091-2098, 1991. 41, 42

[29] R.C. Hudd, A. Jones, and M.N. Kale. Japan Iron and Steel Institute, 209:297301, 1971. 57

[30] F. J. Humphreys. A unified theory of recovery, recrystallization and grain growth, based on the stability and growth of cellular microstructures-I. the basic model. Acta materialia, 45:4231-4240, 1997. 7, 11, 16

[31] F.P. Incropera and D.P. Dewitt. Fundamentals of Heat and Mass Transfer. Wiley, New York, 1985. 42

[32] K.J. Irvine, F.B. Pickering, and T. Gladman. Japan Iron and Steel Institute, 205:161-182, 1976. 57

[33] U. Prahl W. Bleck J. Rudnizki, B. Zeislmair. Prediction of abnormal grain growth during high temperature treatment. Computational Materials Science, 10:209-216, 2010. 7

[34] R. Kampmann and R. Wagner. Decomposition of alloys: the early stages, chapter Kinetics of precipitation in metastable binary alloys - theory and application to Cu-1.9 at\% Ti and Ni-14 at\% Al, pages 91-103. Pergamon Press, 1984. 38

[35] Y. Kang, H.Yu, J. Fu, K. Wang, and Z. Wang. Materials Science and Engineering A, A351:265, 2003. 57

[36] Inderjeet Kaur and Wolfgang Gust. Handbook of Grain and Interphase Boundary Diffusion Data. Stuttgart, 1989, vol 1. 46, 61

[37] Seong Gyoon Kim and Yong Bum Park. Grain boundary segregration, solute drag and abnormal grain growth. Acta Meterialia, 56:3739-3753, 2008. 7, 137

[38] V.K. Lakshmanan and J.S. Kirkaldy. Solubility product for niobium carbide in austenite. Metallurgical Transactions A, 15A:541-544, 1984. 57

[39] C. Leguen, M. Perez, T. Epicier, D. Acevedo, and T. Sourmail. Acta materialia(in review), 2010. 13

[40] Claire Leguen. Prior austenite grain size control by precipitates. PhD thesis, INSA Lyon(Mateis), 2010. 3, 7

[41] W.C. Leslie, R.L. Rickett, C. L. Dotson, and C.S. Walton. Solution and precipitation of aluminium nitride in relation to the structure of low-carbon steels. Trans. ASM, 46:1470-1499, 1954. 57

[42] David San Martín, Francisca G. Caballero, Carlos Capdevila, and Carlos García de Andrés. Austenite grain coarsening under the influence of niobium carbonitrides. Materials Transactions, 45:2797-2804, 2004. 70, 71, 100, 101 
[43] P. Maugis and M. Gouné. Kinetics of vanadium carbonitride precipitation in steel: A computer model. Acta Materialia, 53:3359-3367, 2005. 48

[44] M. Militzer, E.B. Hawbolt, T. Ray Meadowcroft, and A. Giumelli. Austenite grain growth kinetics in Al-killed plain carbon steels. Metallurgical and Materials Transactions A, 27(11):3399-3409, 1996. 70, 71, 100, 101

[45] J. Moon, J. Lee, and C. Lee. Prediction for the austenite grain size in the presence of growing particles in the weld haz of Ti-microalloyed steel. Materials Science \& Engineering A, 459(1-2):40-46, 2007. 75, 104

[46] K. Narita. Physical chemestry of the groups IVa (Ti,Zr), Va (V,Nb,Ta) and the rare earth elements in steel. Transaction ISIJ, 15:145-152, 1975. 57

[47] K. Narita and S. Koyama. Effects of Mn, Si, Cr and Ni on the solubility and precipitation of $\mathrm{NbC}$ in $\mathrm{Fe}$ austenite. Japan Iron and Steel Institute, 35.11:1089-1094, 1971. 57

[48] H. Nordberg and B. Aaronsson. Japan, 206:1263-67, 1968. 57

[49] David L. Olmsted, Stephen M. Foiles, and Elizabeth A. Holm. Survey of computed grain boundary properties in face-centered cubic metals: I. grain boundary energy. Acta Materialia, 57:3694-3703, 2009. 137

[50] W. Scholz P. König and H. Ulmer. Arch. Eisenhüttenwesen, 32 (8):541, 1961. 57

[51] M. Perez. Gibbs-Thomson effect in phase transformations. Scripta Materialia, 52:709-712, 2005. 39

[52] M. Perez, M. Dumont, and D. Acevedo-Reyes. Corrigendum to "Implementation of classical nucleation and growth theories for precipitation". Acta Materialia, 56:2119 Ü 2132, 2008. 48

[53] Mohammad Abdur Razzak, Michel Perez, Thomas Sourmail, Sophie Cazottes, and Marion Frotey. A simple model for abnormal grain growth. ISIJ international, 52(12):2278-2282, 2012. 7

[54] PR Rios. Overview no-62 - A theory for grain-boundary pinning by particles. Acta Metallurgica, 35(12):2805-2814, December 1987. 10

[55] M. Schmid R.M. Tsong and, C. Nagl, P. Yarga, R.F. Davis, and I.S.T. Tsong. Scanning tunneling microscopy studies of niobium carbide (100) and (110) surfaces. Surface Science, 366:86-92, 1996. 61

[56] A. D. Rollett and W. Mullins. On the growth of abnormal grains. Scripta Materialia, 36:975-980, 1997. 6, 16

[57] A.D. Rollett, D.J. Srolovitz, and P. Anderson. Simulation and theory of abnormal grain growth-anisotropic grain boundary energies and mobilities. Acta metallurgica, 37(4):1227-1240, 1989. 7 
[58] K. C. Russell. Phase transformations, chapter Nucleation in solids, pages 219-268. American society for metals, 1968. 38

[59] M. Salamon, S. Dorfman, D. Fuks, G. Inden, and H. Mehrer. Interdiffusion and diffusion of al in iron-aluminides. Diffusion and Defect Data-Solid State Data, Pt. A: Defect and Diffusion Forum, 194-199:553-558, 2001. 46, 61

[60] M. Sennour and C. Esnouf. Contribution of advanced microscopy techniques to nano-precipitates characterization: case of AlN precipitation in low-carbon steel. Acta Materialia, 51:943-957, 2003. 61

[61] T. Shimose and K. Narita:. Japan Iron and Steel Institute, 40:242, 1954. 57

[62] Thomas Skidmore, Rudy G. Buchheit, and Mary C. Juhas. Grain boundary energy vs. misorientation in inconel 600 alloy as measured by thermal groove and oim analysis correlation. Scripta Materialia, 50:873-877, 2004. 137

[63] M. V. Smoluchowski. Versuch einer mathematischen theorie der koagulationskinetik kolloiderlösnngen. Zeitschrift fuer physikalische Chemie, 92:129-168, 1917. 71,101

[64] C. V. Thompson, H.J. Frost, and F. Spaepen. The relative rates of secondary and normal grain growth. Acta Metallurgica, 35:887-890, 1987. 1, 2

[65] S. W. Thompson and G. Krauss. Precipitation and fine structure in mediumcarbon vanadium and vanadium-niobium microalloyed steels. Metallurgical Transactions A, 20A:2279-2288, 1989. 117

[66] W. Thomson. On the equilibrium of vapour at a curved surface of liquid. Philosophical Magazine, 42:448-452, 1871. 38

[67] M. Volmer and A. Weber. Keimbildung in übersättigen gebilden. Z. Phys. Chem., 119:277-301, 1926. 37

[68] R. Wagner and R. Kampmann. Materials science and technology: a comprehensive treatment, volume 5, chapter Homogeneous second phase precipitation, pages 213-302. John Wiley \& Sons Inc, 1991. 213p. 34

[69] Kun Xu and Brian G. Thomas. Prediction of grain size, precipitation and crack susceptibility in continuous casting. In AISTech 2009 Steelmaking Conference Proc., 2009. 70, 71, 100, 101

[70] Y. B. Zeldovich. On the theory of new phase formation: Cavitation. Acta Physicochim USSR, 18:1-22, 1943. 37

[71] C. Zener. Theory of growth of spherical precipitates from solid solution. Journal of Applied Physics, 20:950-953, 1949. 7, 39, 44

[72] Liang Zhang and Dong Cheng Guo. A general etchant for revealing prioraustenite grain boundaries in steels. Materials Characterization, 30:299-305, 1993. 140 